Florida International University FIU Digital Commons

\title{
Study of the Transport of Odorants from Illicit Substances Using Direct Analysis in Real Time Mass Spectrometry
}

Torki A. Zughaibi

Florida International University, torkiaz@gmail.com

DOI: $10.25148 /$ etd.FIDC001940

Follow this and additional works at: https://digitalcommons.fiu.edu/etd

\section{Recommended Citation}

Zughaibi, Torki A., "Study of the Transport of Odorants from Illicit Substances Using Direct Analysis in Real Time Mass Spectrometry" (2017). FIU Electronic Theses and Dissertations. 3398.

https://digitalcommons.fiu.edu/etd/3398 


\title{
FLORIDA INTERNATIONAL UNIVERSITY
}

\author{
Miami, Florida
}

STUDY OF THE TRANSPORT OF ODORANTS FROM ILLICIT SUBSTANCES USING DIRECT ANALYSIS IN REAL TIME MASS SPECTROMETRY

A dissertation submitted in partial fulfillment of

the requirements for the degree of

DOCTOR OF PHILOSOPHY

in

CHEMISTRY

by

Torki A. Zughaibi 
To: Dean Michael R. Heithaus

College of Arts, Sciences and Education

This dissertation, written by Torki A. Zughaibi, and entitled Study of the Transport of Odorants from Illicit Substances Using Direct Analysis in Real Time Mass Spectrometry, having been approved in respect to style and intellectual content, is referred to you for judgment.

We have read this dissertation and recommend that it be approved.

$\begin{array}{r}\hline \text { Alexander Mebel } \\ \hline \text { Yong Cai } \\ \hline \text { Stewart D'Alessio } \\ \hline \text { Kenneth G. Furton, Major Professor }\end{array}$

Date of Defense: June 29, 2017

The dissertation of Torki A. Zughaibi is approved.

Dean Michael R. Heithaus

College of Arts, Sciences and Education

Andrés G. Gil

Vice President for Research and Economic Development

And Dean of the University Graduate School

Florida International University, 2017 
(C) Copyright 2017 by Torki A. Zughaibi

All rights reserved. 


\section{DEDICATION}

I would like to dedicate this dissertation to my parents, Dr. Hana Al-Nuaim and Dr. Abdulwahab Al-Zughaibi, my siblings, Lama, Nasser and Jude and my wife, Nabia Ali. Thank you for all your support throughout my life and for dealing with me when I needed it most. 


\section{ACKNOWLEDGMENTS}

I would first like to thank King Abdul-Aziz University for their financial support towards this research project. In addition, I would like to thank Virginia Commonwealth University's Forensic Science Department for their collaboration and allowing me to use their facilities and instrument for this research. I would also like to thank my research mentor Dr. Kenneth G. Furton for all his help and expertise, and especially for providing me with the opportunity to join his research team. But most importantly, thank you for taking the time to first meet me on a random Sunday in the summer of 2011 on your way back from the airport! To my committee members, Dr. Alexander Mebel, Dr. Yong Cai and Dr. Stewart D’Alessio, thank you for your support and your suggestions, and for your guidance throughout this project. Last, but not least, thank you Dr. Michelle Peace for everything you have done since we met in my freshman year in undergrad, over 10 years ago! You promised you would do your best to help me achieve my goals, and with your support, I was able to attend VCU's M.S. in Forensic Science program like we discussed during our first meeting. Thank you for also accepting this role to be on my committee, and helping me with the opportunity to complete my research where it all started.

For my VCU family, thank you all for the good memories and the fun moments throughout my six years in Richmond. Thank you to John Benson for your help with my research in Richmond, two sets of hands really made this project move quicker! To Dr. Tracey Dawson-Cruz, thank you for taking a chance with me by accepting me into the Master's program on graduation day! It just made that day even more special. To Dr. Marilyn Miller, thank you for your mentorship during grad school and for opening the door 
to research by vouching for me in order to be an intern with Mr. Bob Steiner at the Virginia Department of Forensic Science. Without question, those three months as an intern and doing research proved to be the most fulfilling and informative experiences I've ever had. Thank you to Mr. Bob Steiner, for your mentorship, guidance, and friendship. I can confidently say that without that experience working with you, this doctoral research wouldn't have been possible. Thank you for pushing me, encouraging me and teaching me all you could during our short time together.

To my FIU family, thank you so much for the good memories in Miami during my time there. You made the experience a lot more enjoyable with your companionship, and I hope I have left a positive impression on all of you. For the Dr. Furton lab group, Philip, Rudy, Iris, Michelle, Jessica, Danay, Lauren, Claudia, Adhly and Van, thank you all so much for all the great memories we've had in Miami and in Seattle, Orlando, Washington D.C. and New Orleans. I hope you all remember me every time you have a delicious meal! To Van and Adhly, thank you so much for your help with my research, I am eternally grateful for your support.

Finally, I would like to thank Dr. Howard Holness, for your friendship and guidance from my early days in Miami, and for taking care of my baby (my car) over the summer. More importantly, thank you for your time, effort and assistance throughout my research and for your suggestions to try different approaches whenever I hit a dead end. 


\section{ABSTRACT OF THE DISSERTATION}

\section{STUDY OF THE TRANSPORT OF ODORANTS FROM ILLICIT SUBSTANCES}

\section{USING DIRECT ANALYSIS IN REAL TIME MASS SPECTROMETRY}

by

\section{Torki A. Zughaibi}

Florida International University, 2017

Miami, Florida

Professor Kenneth G. Furton, Major Professor

Canines have been employed in matters of law enforcement because of their keen sense of smell. Presently, law enforcement officials are utilizing trained canines in routine traffic stops to assess if the vehicle contains any illicit substances. Many believe that this is an infringement on an individual's fourth amendment rights, which has garnered the attention of both the media and the courts. Many questions have been raised with respect to canines alerting to locations where illicit substances were no longer present. Thus, the purpose of this dissertation research is to evaluate the manner in which active odorants transport and persist onto various substrates.

Direct Analysis in Real Time (DART) coupled to an accurate-mass time-of-flight (AccuTOFTM) mass spectrometer was used to rapidly analyze the volatile organic compounds (VOC's) from a variety of narcotic and explosive substances. The DART ion source is a soft ionization technique used in ambient conditions to sample liquids, solids or gases in real time. Thermal desorption of the VOC's could thus be conducted in seconds. 
The present study found that the VOC's from illicit substances transport from one location to another, in a short amount of time, through a process known as advection, which may contribute to canines producing unconfirmed alerts during their training and certifications. Three of the four odorants used in this study produced positive results, with the exception being 2-ethyl-1-hexanol, as it was not detected at any time when held at distances between 0.5 and 3 meters.

In addition, the amount of time needed for an active odorant to contaminate an object in its immediate vicinity was explored and the results were determined to be inconclusive. Although they were observed following longer exposure times, it was still deemed to be inconclusive since it was still possible for these odorants to be present, albeit not in detectable amounts, at lower exposure times.

Controlled odor mimic permeating systems (COMPS), patented technology by IFRI were tested to determine the possibility of cross-contamination between the training aids, and the results conclude that there was not any evidence of cross-contamination observed during any of the trials. 


\section{TABLE OF CONTENTS}

\section{CHAPTER}

\section{PAGE}

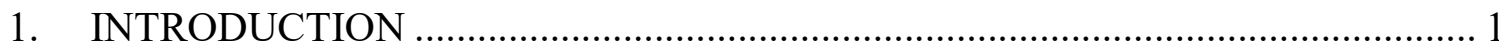

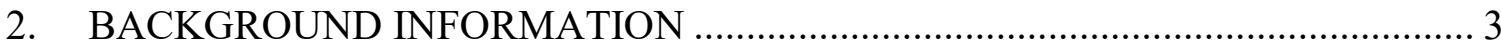

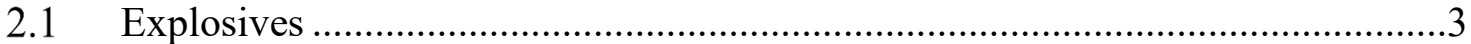

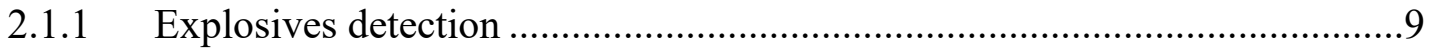

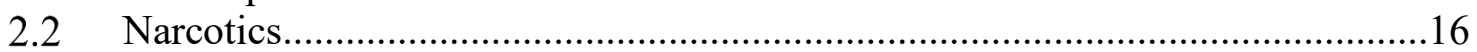

2.3 Detection of Illicit Substances Outside of the Laboratory …...............................26

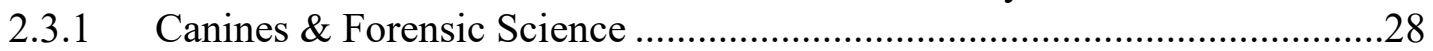

2.3.2 The Anatomy of the Canine Nose and its Olfactory System .......................29

2.3.3 Canines \& The Justice System....................................................................32

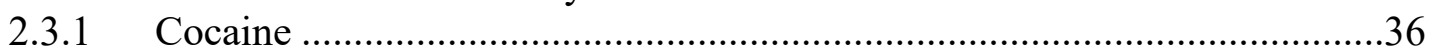

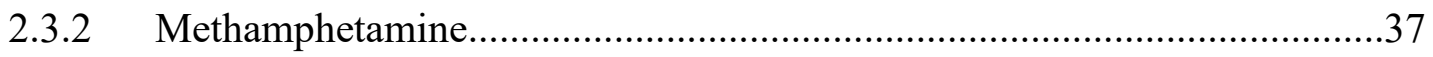

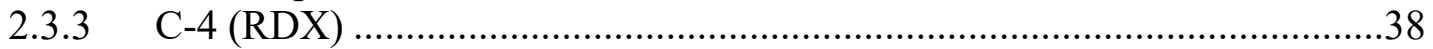

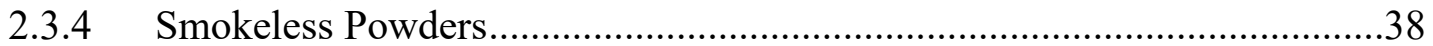

2.3.5 Volatile Organic Compounds and Residual Odors......................................39

2.3.6 Training Aids and Controlled Odor Mimic Permeation Systems (COMPS)

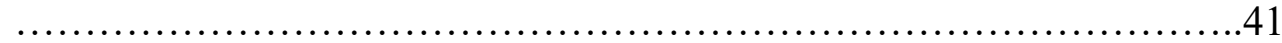

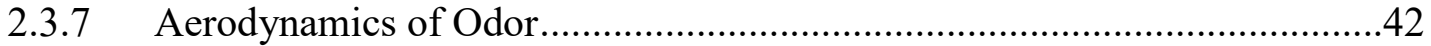

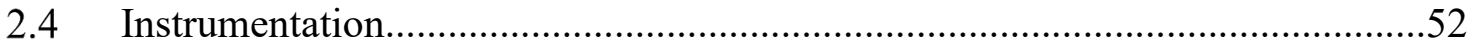

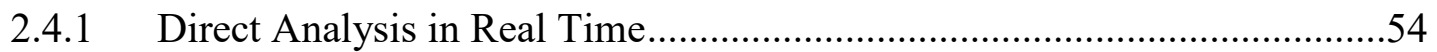

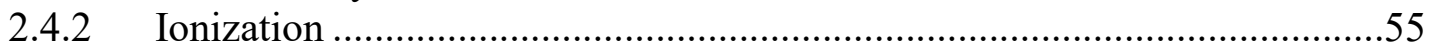

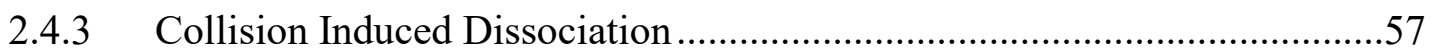

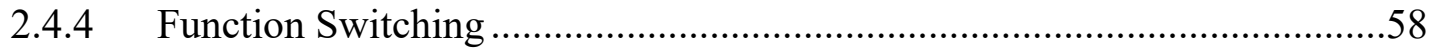

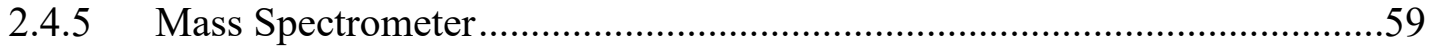

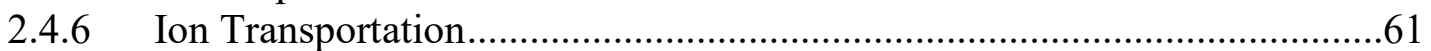

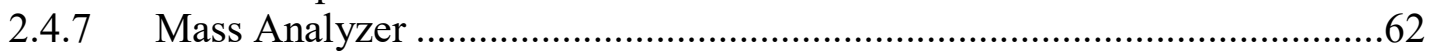

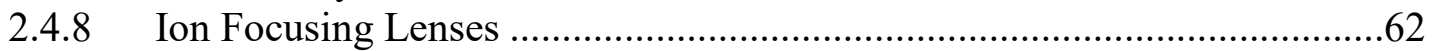

2.4.9 Time-of-Flight Mass Analyzer …………………...................................63

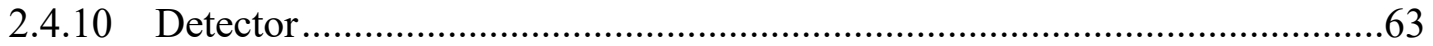

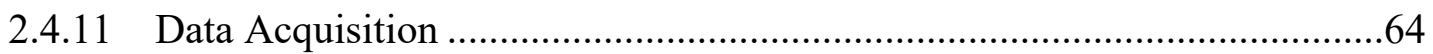

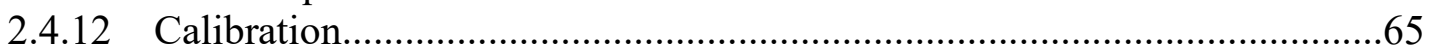

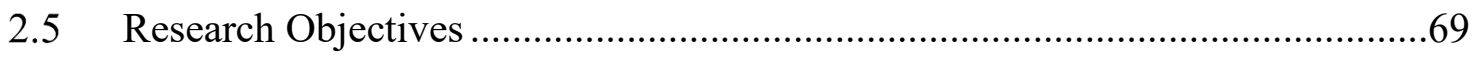

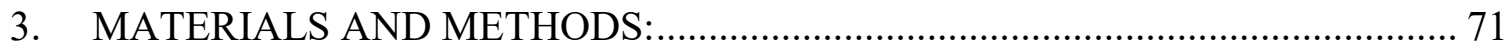

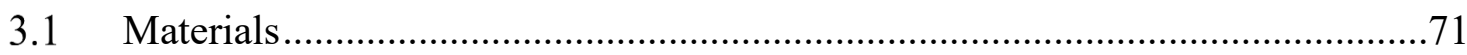

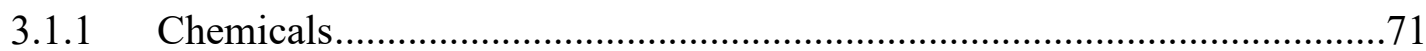

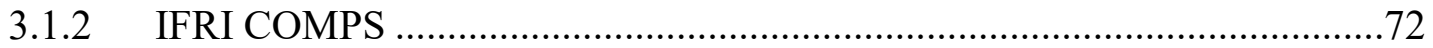

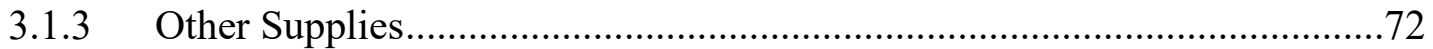

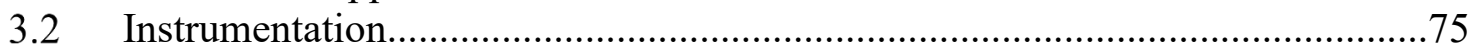


3.2.1 Direct Analysis in Real Time - Accurate Time of Flight Mass Spectrometer

\subsection{Methodology} . .75

3.3.1 Task 1: Optimizing DART Parameters and Identification of Chemical

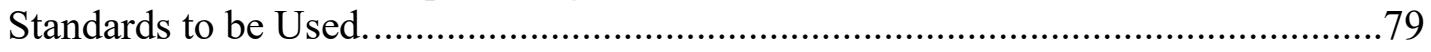

3.3.2 Task 2: Evaluating the Speed of VOC Transport ........................................83

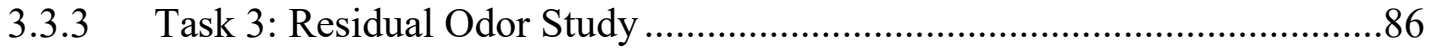

3.3.4 Task 4: Testing COMPS' Potential for Cross-Contamination......................92

3.3.5 Task 5: Survey of Narcotic and Explosive Detecting Canine Handlers ......97

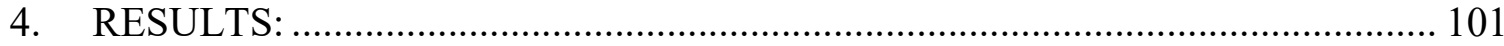

4.1 Task 1: Optimizing DART Parameters ……………………………................101

4.2 Task 2: Evaluating the Speed of VOC Transport...............................................119

4.3 Task 3: Residual Odor Study ………………................................................ 141

4.4 Task 4: Testing COMPS' Potential for Cross-Contamination............................176

4.5 Task 5: Survey of Narcotic and Explosive Detecting Canine Handlers ...........183

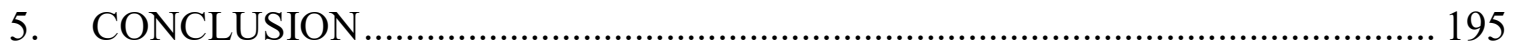

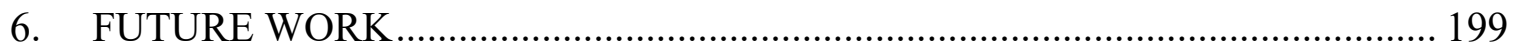

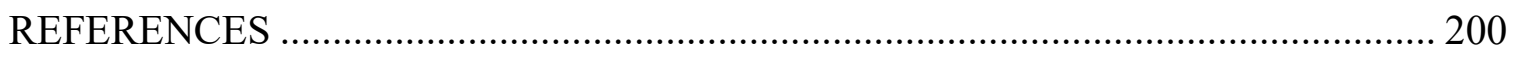

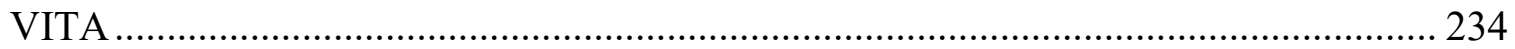




\section{LIST OF TABLES}

TABLE

PAGE

Table 1 - Techniques recommended for post-blast forensic analysis as suggested by T/SWGFEX[10].

Table 2 - Summary of common controlled substances and their DEA schedule and corresponding penalties [14]

Table 3 - DEA drug seizure amounts from 2003-2013 [15].

Table 4 - The major categories of analytical techniques as suggested by SWGDRUG [17].

Table 5 - Results of the most common spot tests and their expected color change [18].

Table 6 - Examples of illicit substances and their primary odors [2], [21], [48]-[50]... 36

Table 7 - Compounds found in headspace of smokeless powders and their detection mode [54].

Table 8 - Examples of odorants, their molecular formula, weight and structures.

Table 9 - Exact mass of each polyethylene glycol (PEG) peak [90].

Table 10 - This table describes how the experiment for Task 3, Part C was planned. (nd) $=$ next day.

Table 11 - Average response of dibutyl phthalate (DBP) and diethyl phthalate (DEP) at temperatures ranging from $150^{\circ} \mathrm{C}-500^{\circ} \mathrm{C}$.

Table 12 - Averaged responses (x1000) for the four standards at temperatures $150^{\circ}$ $500^{\circ} \mathrm{C}$ at $50^{\circ} \mathrm{C}$ intervals.

Table 13 - Summary of results for methyl benzoate at $0.5 \mathrm{~m}$

Table 14 - Summary of results for methyl benzoate at $1 \mathrm{~m}$.

Table 15 - Summary of results for methyl benzoate at a distance of $1.5 \mathrm{~m}$. 128

Table 16 - Summary of results for methyl benzoate at a distance of $2 \mathrm{~m}$. 128

Table 17 - Summary of results for methyl benzoate at a distance of $3 \mathrm{~m}$. 128 
Table 18 - Summary of results for Benzaldehyde at all distances.

Table 19 - Summary of results for 2-ethyl-1-hexanol at all distances. (DND = Did not detect, N/A = Not applicable)

Table 20 - Summary of results for diethyl phthalate at all distances. (DND $=$ Did not

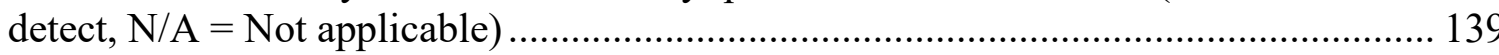

Table 21 - Summary of averaged times for all four chemical standards..................... 140

Table 22 - Results for Task 3, Part A using a large plastic container. (DND = Did not detect).

Table 23 - Intensities for samples tested for methyl benzoate toothpick experiment. Along with the calculated average intensity at each 2 hour interval and its standard deviation.

Table 24 - Intensities for samples tested for methyl benzoate glass experiment. Along with the calculated average intensity at each 2 hour interval and its standard deviation.

Table 25 - Intensities for samples tested for benzaldehyde toothpick experiment. Along with the calculated average intensity at each 2 hour interval and its standard deviation.

Table 26 - Intensities for samples tested for benzaldehyde capillary glass experiment. Along with the calculated average intensity at each 2 hour interval and its standard deviation.

Table 27 - Intensities for samples tested for 2-ethyl-1-hexanol toothpick experiment. Along with the calculated average intensity at each 2 hour interval and its standard deviation.

Table 28 - Intensities for samples tested for diethyl phthalate toothpick experiment. Along with the calculated average intensity at each 2 hour interval and its standard deviation.

Table 29 - Intensities for samples tested for diethyl phthalate glass experiment. Along with the calculated average intensity at each 2 hour interval and its standard deviation.

Table 30 - Intensities for samples tested for diethyl phthalate metal experiment. Along with the calculated average intensity at each 2 hour interval and its standard deviation.

Table 31 - Results for narcotic COMPS secondary container cross-contamination test. 
Table 32 - Results for narcotic COMPS primary container cross-contamination test... 178

Table 33 - Results for explosive COMPS secondary container cross-contamination tests. 


\section{LIST OF FIGURES}

FIGURE

PAGE

Figure 1 - A family of explosives with molecular backbone of 1,3,5 trinitrobenzene [6].

Figure 2 - The three most important parameters for explosive detection systems [5].... 12

Figure 3 - Number of drug related domestic arrests from 2005-2015 [15] ................... 20

Figure 4 - Illustrating the trend between yearly drug seizures of marijuana versus

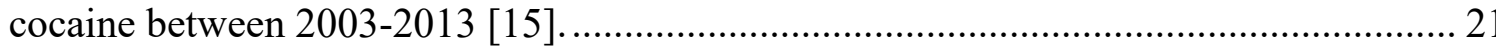

Figure 5 - Illustrating the trend between yearly drug seizures of heroin versus

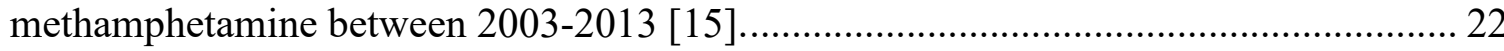

Figure 6 - Example of a TLC plate. ...................................................................... 25

Figure 7 - A chart illustrating the several detection methods for trace explosive detection currently available and also under development [21].

Figure 8 - (a) Is a 3D model of the left side of the canine nasal airway and (b) shows

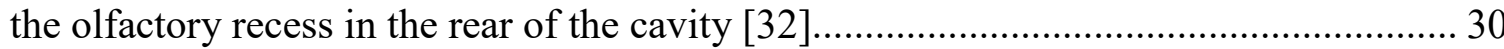

Figure 9 - Illustration of human olfactory system that describes the steps between breathing in odors to brain signal [33].

Figure 10 - Difference in chemical structure between cocaine and one of its volatile byproducts, methyl benzoate

Figure 11 - Comparison of cocaine headspace response by different Solid Phase Microextraction (SPME) fibers, at an exposure time of 12 hours [44].

Figure 12 - Headspace analysis of untagged and tagged C-4. Peak 1 is 2-ethyl-1hexanol and peak 2 is 2,3-dimethyl-dinitrobutane [21]

Figure 13 - Picture of the narcotic COMPS made at FIU.

Figure 14 - Graph of Péclet number that illustrates when diffusion dominates advection in its dispersion and vice versa [29]. 46

Figure 15 - Graph illustrating airflow velocities within a boundary layer [29]. 50

Figure 16 - Picture representing the three distinct phases observed in the distribution of an odor plume. The black lines represent the boundaries of the plume as well as the 
individual odor filaments within the plume. The blue swirls represent airflow eddies [29].

Figure 17 - Schematic diagram of the DART ion source [66].

Figure 18 - (a) Particle tracing simulation of circular acetaminophen tablet at simulated particle velocities $(\mathrm{m} / \mathrm{s})$ and $(\mathrm{b})$ temperatures and scale $\left({ }^{\circ} \mathrm{C}\right)$. Top row: Above position: (i) sideways, (ii) flat and (iii) upright. Middle row: Inline position: (iv) sideways, (v) flat and (vi) upright. Bottom row: Below position (vii) sideways, (viii) flat and (ix) upright [85].

Figure 19 - The different fragment ions created in scopolamine and cocaine. 58

Figure 20 - Mass spectra for scopolamine (top) showing the fragment ions that would distinguish it from an unknown vs. cocaine (bottom) [68].

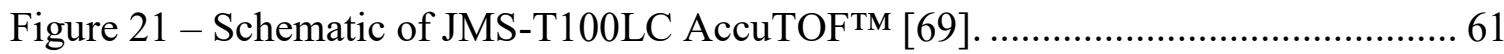

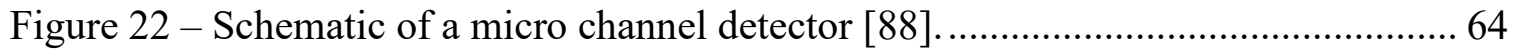

Figure 23 - PEG 600 positive ion mode reference mass spectrum [89] ......................... 66

Figure 24 - Total ion response of nylon 6/9; (a) $200^{\circ} \mathrm{C}$, (b) $225^{\circ} \mathrm{C}$, (c) $250^{\circ} \mathrm{C}$, (d) $275^{\circ} \mathrm{C}$ and (e) $300^{\circ} \mathrm{C}$.

Figure 25 - DART-AccuTOF ${ }^{\mathrm{TM}}$ mass spectra for nylon 6 at orifice voltage of (a) 20 volts, (b) 30 volts and (c) 60 volts.

Figure 26 - DART-AccuTOF ${ }^{\mathrm{TM}}$ mass spectra for nylon $6 / 6$ at orifice voltage of (a) 20 volts, (b) 30 volts and (c) 60 volts.

Figure 27 - DART-AccuTOFTM mass spectra for standard nylon 6 at 20 volts with correct peaks identified using SearchFromList software.

Figure 28 - DART-AccuTOFTM at the Virginia Commonwealth University laboratory.

Figure 29 - Settings pane in MS Tune Manager, where orifice 1 voltage, among other parameters, can be chosen.

Figure 30 - DART SVP software. Temperature can be chosen in $50^{\circ} \mathrm{C}$ intervals from the dropdown menu where "Heater Off" is located.

Figure 31 - Manner in which headspace of liquid samples are analyzed using the DART. 
Figure 32 - Layout of the room where the DART-AccuTOFTM (A) is located. Where $\mathrm{B}$ are desks, $\mathrm{C}$ is the computer desk, $\mathrm{D}$ are the tanks, and $\mathrm{E}$ is an air conditioning unit. (Drawing not to scale).

Figure 33 - Example showing the different RIC's. Top RIC shows what the RIC looks like after CODA is performed, while the middle RIC is the before CODA, and the bottom is the RIC created using only the $\mathrm{m} / \mathrm{z}$ information selected from the mass spectrum (right). In this case, the bottom RIC shows the responses where only $107 \mathrm{~m} / \mathrm{z}$ was identified.

Figure 34 - RIC of $107 \mathrm{~m} / \mathrm{z}$. Red arrow was added by user to indicate the time when the vial was opened. Selected area is the first peak that identified detection of benzaldehyde.

Figure 35 - Example of RIC where metal is ionized, creating a 'negative' peak, which artificially raises the baseline.

Figure 36 - Picture of the large plastic container used in experiment Task 3, Part A. In

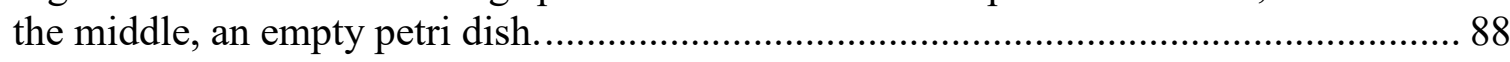

Figure 37 - Example set up for Task 3, Part C....................................................... 89

Figure 38 - Example of one of the Glad ${ }^{\circledR}$ plastic containers with 6 toothpicks, 6 glass capillary tubes (broken) and 6 metal clips. The number of substrates indicate that 2 exposure times will be tested using this container ( 3 samples of each substrate for each exposure time).

Figure 39 - Sampling the exterior of the mason jars.

Figure 40 - Picture of the primary (right) and secondary (left) containers used for the narcotic mimics.

Figure 41 - Sampling of the LDPE bags. This needed to be carefully done so that the DART stream does not melt the bag and expose its contents.

Figure $42-\mathrm{RIC}$ of methyl benzoate $300^{\circ} \mathrm{C}-150^{\circ} \mathrm{C}$.

Figure 43 - RIC of methyl benzoate $350^{\circ} \mathrm{C}-500^{\circ} \mathrm{C}$

Figure $44-\mathrm{RIC}$ of benzaldehyde $300^{\circ} \mathrm{C}-150^{\circ} \mathrm{C}$

Figure 45 - RIC of benzaldehyde $350^{\circ} \mathrm{C}-500^{\circ} \mathrm{C}$.

Figure 46 - RIC of 2-ethyl-1-hexanol $300^{\circ} \mathrm{C}-150^{\circ} \mathrm{C}$ 104

Figure 47 - RIC of 2-ethyl-1-hexanol $350^{\circ} \mathrm{C}-500^{\circ} \mathrm{C}$ 104 
Figure 48 - RIC of diethyl phthalate $300^{\circ} \mathrm{C}-150^{\circ} \mathrm{C}$. 105

Figure 49 - RIC of diethyl phthalate $350^{\circ} \mathrm{C}-500^{\circ} \mathrm{C}$. 105

Figure 50 - Mass spectra of methyl benzoate at various temperatures $\left(150^{\circ} \mathrm{C}-300^{\circ}\right) \ldots 107$

Figure 51 - Mass spectra of methyl benzoate at various temperatures $\left(350^{\circ} \mathrm{C}-500^{\circ}\right)$.. 108

Figure 52 - Mass spectra of benzaldehyde at various temperatures $\left(150^{\circ} \mathrm{C}-300^{\circ}\right)$....... 109

Figure 53 - Mass spectra of benzaldehyde at the various temperatures $\left(350^{\circ} \mathrm{C}-500^{\circ}\right) . .110$

Figure 54 - Mass spectrum of 2-ethyl-1-hexanol at $150^{\circ} \mathrm{C}(30 \mathrm{~V}) \ldots \ldots \ldots \ldots \ldots \ldots \ldots \ldots \ldots \ldots . . . . .111$

Figure 55 - 2-ethyl-1-hexanol dimer. ...................................................................... 111

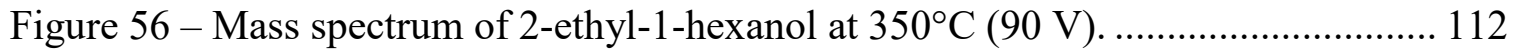

Figure 57 - Mass spectra of 2-ethyl-1-hexanol at temperatures of $200^{\circ} \mathrm{C}$ (top), $250^{\circ} \mathrm{C}$

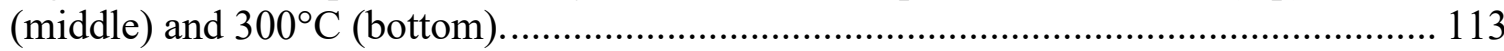

Figure 58 - Mass spectrum of 2,4-dinitrotoluene in positive ion mode at $350^{\circ} \mathrm{C}(30$ V).

Figure 59 - Mass spectrum of 2,4-dinitrotoluene in negative ion mode at $350^{\circ} \mathrm{C}(30$ V).

Figure 60 - Mass spectrum for dibutyl phthalate with molecular ion peak $(279 \mathrm{~m} / \mathrm{z})$ present but at a very low intensity (at $\left.500^{\circ} \mathrm{C}\right)$.

Figure 61 - Mass spectrum for dibutyl phthalate where the molecular ion peak (279 $\mathrm{m} / \mathrm{z}$ ) was not observed $\left(\right.$ at $200^{\circ} \mathrm{C}$ ).

Figure 62 - Mass spectra of diethyl phthalate at $400^{\circ} \mathrm{C}$ (top), $450^{\circ} \mathrm{C}$ (middle) and $500^{\circ} \mathrm{C}$ (bottom).

Figure 63 - Visual representation of the data from Table 12 - Averaged responses (x1000) for the four standards at temperatures $150^{\circ}-500^{\circ} \mathrm{C}$ at $50^{\circ} \mathrm{C}$ intervals.

Figure 64 - RIC of methyl benzoate (trial \#1) at a distance of $0.5 \mathrm{~m}$.

Figure 65 - RIC's of methyl benzoate at a distance of $0.5 \mathrm{~m}$ (Trial \#1). Top: RIC after performing CODA (zoomed in). Bottom: RIC showing only peaks where $137 \mathrm{~m} / \mathrm{z}$ was detected. Cap opened at 1:02 minutes

Figure 66 - Mass spectrum of methyl benzoate (trial $\# 1$ ) at distance $0.5 \mathrm{~m}$, first detected at 1.12 minutes. 
Figure 67 - RIC's of methyl benzoate at a distance of $0.5 \mathrm{~m}$ (Trial \#2). Top: RIC after performing CODA (zoomed in). Bottom: RIC showing only peaks where $137 \mathrm{~m} / \mathrm{z}$ was detected. Cap opened at 1:02 minutes.

Figure 68 - Mass spectrum of methyl benzoate (trial \#2) at distance $0.5 \mathrm{~m}$, first detected at $1.18(1: 10)$ minutes.

Figure 69 - RIC's of methyl benzoate at a distance of $0.5 \mathrm{~m}$ (Trial \#2). Top: RIC after performing CODA (zoomed in). Bottom: RIC showing only peaks where $137 \mathrm{~m} / \mathrm{z}$ was detected. Cap opened at 1:03 minutes

Figure 70 - Mass spectrum of methyl benzoate (trial \#3) at distance $0.5 \mathrm{~m}$, first detected at $1.13(1: 08)$ minutes.

Figure 71 - RIC's of methyl benzoate at a distance of 1m (Trial \#1). Top: RIC after performing CODA (zoomed in). Bottom: RIC showing only peaks where $137 \mathrm{~m} / \mathrm{z}$ was detected. Cap opened at 56 seconds.

Figure 72 - Mass spectrum of methyl benzoate (trial \#1) at distance 1m, first detected at $1.18(1: 11)$ minutes.

Figure 73 - RIC's of methyl benzoate at a distance of $1 \mathrm{~m}$ (Trial \#2). Top: RIC after performing CODA (zoomed in). Bottom: RIC showing only peaks where $137 \mathrm{~m} / \mathrm{z}$ was detected. Cap opened at 1 minute.

Figure 74 - Mass spectrum of methyl benzoate (trial \#2) at distance 1m, first detected at $1.37(1: 22)$ minutes.

Figure 75 - RIC's of methyl benzoate at a distance of 1m (Trial \#3). Top: RIC after performing CODA (zoomed in). Bottom: RIC showing only peaks where $137 \mathrm{~m} / \mathrm{z}$ was detected. Cap opened at 1 minute and 1 second (1:01)....

Figure 76 - Mass spectrum of methyl benzoate (trial \#3) at distance 1m, first detected at $1.4(1: 24)$ minutes.

Figure 77 - Averaged results of task 2 study for methyl benzoate at the various distances.

Figure 78 - RIC's of benzaldehyde at a distance of $2 \mathrm{~m}$ (Trial \#2). Top: RIC after performing CODA (zoomed in). Bottom: RIC showing only peaks where $107 \mathrm{~m} / \mathrm{z}$ was detected. Cap opened at 1 minute and 6 seconds (1:06 min).

Figure 79 - Mass spectrum of benzaldehyde (trial \#2) at distance $2 \mathrm{~m}$, first detected at $1.84(1: 50)$ minutes.

Figure 80 - Averaged results of task 2 study for benzaldehyde at the various distances. 
Figure 81 - RIC's of 2-ethyl-1-hexanol at a distance of $0.5 \mathrm{~m}$ (Trial \#3). Top: RIC after performing CODA (zoomed in). Bottom: RIC showing only peaks where $261 \mathrm{~m} / \mathrm{z}$ was detected. Cap opened at 58 seconds (0:58 min).....

Figure 82 - Mass spectrum of 2-ethyl-1-hexanol positive control

Figure 83 - RIC's of 2-ethyl-1-hexanol at a distance of 2m (Trial \#1). Top: RIC after performing CODA (zoomed in). Bottom: RIC showing only peaks where $261 \mathrm{~m} / \mathrm{z}$ was detected. Cap opened at 1 minute and 5 seconds (1:05 min).

Figure 84 - RIC's of 2-ethyl-1-hexanol at a distance of 3m (Trial \#3). Top: RIC after performing CODA (zoomed in). Bottom: RIC showing only peaks where $261 \mathrm{~m} / \mathrm{z}$ was detected. Cap opened at 1 minute and 18 seconds (1:18 min).

Figure 85 - RIC's of diethyl phthalate at a distance of $0.5 \mathrm{~m}$ (Trial \#2). Top: RIC after performing CODA (zoomed in). Bottom: RIC showing only peaks where $223 \mathrm{~m} / \mathrm{z}$ was detected. Cap opened at 57 seconds (0:57 min)

Figure 86 - Mass spectrum of diethyl phthalate (trial \#2) at distance $0.5 \mathrm{tm}$, first detected at $1.16(1: 09)$ minutes.

Figure 87 - RIC's of diethyl phthalate at a distance of 1.5m (Trial \#2). Top: RIC after performing CODA (zoomed in). Bottom: RIC showing only peaks where $223 \mathrm{~m} / \mathrm{z}$ was detected. Cap opened at 48 seconds (0:48 min).

Figure 88 - Mass spectrum of diethyl phthalate (trial \#2) at distance 1.5tm, first detected at 2.08 (2:05) minutes. Intensity for $223 \mathrm{~m} / \mathrm{z}$ was above $5 \%$ relative intensity.

Figure 89 - Averaged results of task 2 study for diethyl phthalate at the various distances. No results obtained at distances $2 \mathrm{~m}$ and $3 \mathrm{~m}$.

Figure 90 - Graph comparing the averaged results for the different standards at the different distances

Figure 91 - Picture of the mason jar with the dollar bill exposed to methyl benzoate, placed in a petri dish at the bottom of the jar.

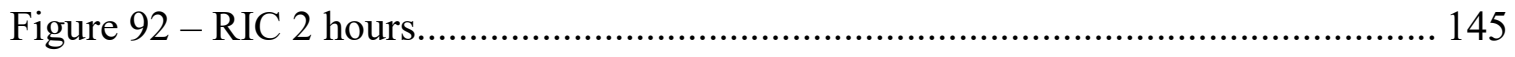

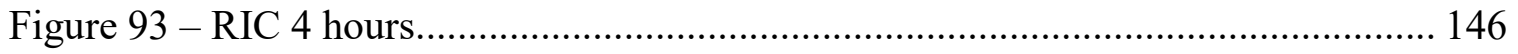

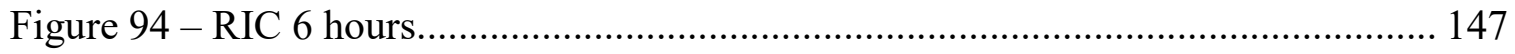

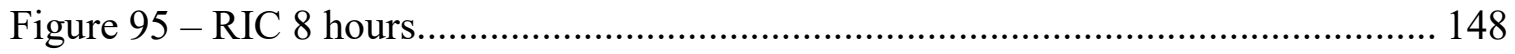

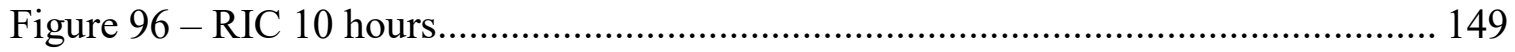




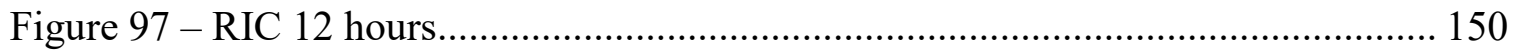

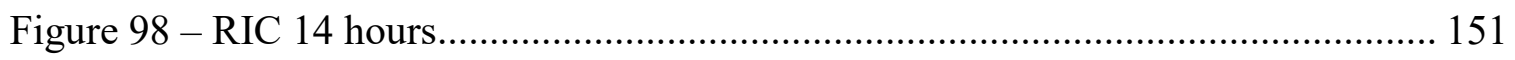

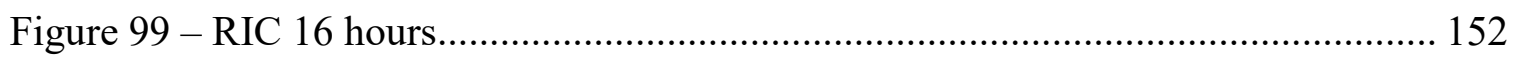

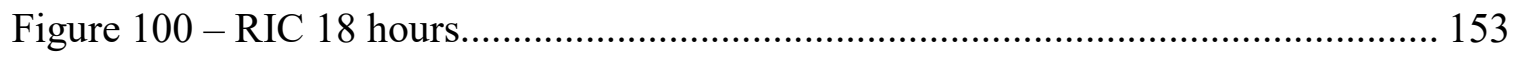

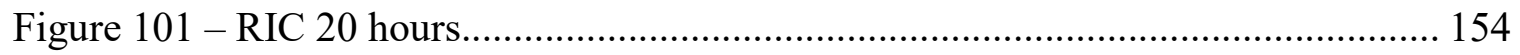

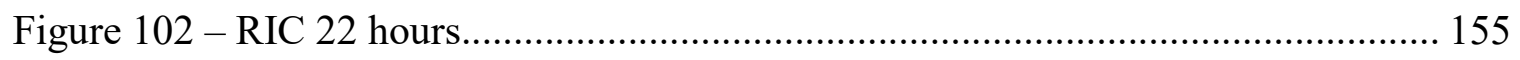

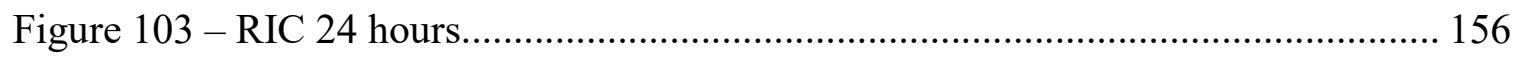

Figure 104 - Visual representation of data from Table 23............................................ 157

Figure 105 - Mass spectrum of methyl benzoate (m/z 137) toothpick sample \#1 (From RIC shown in Figure 92, $4^{\text {th }}$ peak from the left). The intensity is shown at the top (Max Inten)

Figure 106 - Visual representation of data from Table 24 ......................................... 160

Figure 107 - Visual representation of data from Table 25......................................... 161

Figure 108 - Visual representation of data fromTable 26 .......................................... 162

Figure 109 - Mass spectrum of the glass sample \#2 exposed to benzaldehyde $(\mathrm{m} / \mathrm{z}$ 107). (From RIC shown in Figure 97, $18^{\text {th }}$ peak from the left).

Figure 110 - Chart comparing the averaged intensities for toothpick, glass and metal clip data (benzaldehyde).

Figure 111 - Chart comparing results for toothpick, glass and metal clips exposed to 2-ethyl-1-hexanol for 24 hours at 2 hour intervals.

Figure 112 - Mass spectrum of the toothpick sample \#1 exposed to 2-ethyl-1-hexanol for 8 hours. (From RIC shown in Figure 95, $4^{\text {th }}$ peak from the left). Since $\mathrm{m} / \mathrm{z} 261$ is not the parent ion peak in this mass spectrum, its relative intensity is shown on the table to the right of the mass spectrum.

Figure 113 - Visual representation of Table 28 ............................................................ 168

Figure 114 - Mass spectrum of the toothpick sample \#1 exposed to diethyl phthalate for 10 hours. (From RIC shown in Figure 96, $4^{\text {th }}$ peak from the right). Since $\mathrm{m} / \mathrm{z} 233$ is not the parent ion peak in this mass spectrum, its relative intensity is shown on the table to the right of the mass spectrum.

Figure 115 - Visual representation of Table 29 170 
Figure 116 - Visual representation of Table 30.

Figure 117 - Zoomed in RIC for 24 hour exposure experiment (full RIC shown in Figure 103). The dotted lines indicate the area selected by the user to average to create a mass spectrum.

Figure 118 - Mass spectrum of the 2-ethyl-1-hexanol standard in the 24-hour exposure experiment (RIC shown in Figure 103). Max intensity of 131,668. 173

Figure 119 - Zoomed in RIC for 24-hour exposure experiment (full RIC shown in Figure 103). The dotted lines indicate the area selected by the user to average to create a mass spectrum. Note the slight differences between this figure and Figure 117 in the area selected.

Figure 120 - Mass spectrum of the 2-ethyl-1-hexanol standard in the 24-hour exposure experiment (RIC shown in Figure 103). Max intensity of 179,888.

Figure 121 - TOP: RIC for methamphetamine COMPS secondary container test. First series of peaks are PEG calibrant peaks. Last series of peaks are benzaldehyde standard. Bottom: RIC for $\mathrm{m} / \mathrm{z} 107$ that shows it was not detected at any point during the run, with the exception of the standard.

Figure 122 - TOP: RIC for cocaine COMPS secondary container test. First series of peaks are PEG calibrant peaks. Last series of peaks are methyl benzoate standard. Bottom: RIC for $\mathrm{m} / \mathrm{z} 137$ that shows it was detected at various points during the run. . 178

Figure 123 - TOP: RIC for smokeless powder \#1 COMPS secondary container test (first trial). First series of peaks are PEG calibrant peaks. Last series of peaks are sampling the LDPE bag containing methyl centralite standard. Bottom: RIC for $\mathrm{m} / \mathrm{z}$ 269 that shows it was detected at various points during the run.

Figure 124 - TOP: RIC for smokeless powder \#2 COMPS secondary container test (first trial). First series of peaks are PEG calibrant peaks. Last series of peaks are sampling the LDPE bags containing diphenyl amine standard. Bottom: RIC for $\mathrm{m} / \mathrm{z}$ 170 that shows it was detected at various points during the run.

Figure 125 - TOP: RIC for smokeless powder \#2 COMPS secondary container test (second trial). First series of peaks are PEG calibrant peaks. Last series of peaks are sampling the LDPE bags containing diphenyl amine standard. Bottom: RIC for $\mathrm{m} / \mathrm{z}$ 170 that shows it was detected at various points during the run......

Figure 126 - 1. Do you work for a County, State or Federal Agency?

Figure 127 - 2. Do you use commercially available training aids, in-house prepared pseudo scent training aids, or actual drugs/explosives to train your canine teams? ....... 185 
Figure $128-4$. Have you observed unconfirmed alerts that can be attributed to the close proximity of targets to illicit substances?

Figure 129 - 5. Have you observed unconfirmed alerts that can be attributed to potential storage issues?

Figure $130-6$. Do you believe unconfirmed alerts can be attributed to an illicit substance recently being present in that particular location?...

Figure $131-7$. Would you be willing to participate in a scientific study testing new training aids for effectiveness?

Figure $132-8$. How are the training aids contained or stored between training? ......... 189

Figure $133-10$. How often are your training aids changed to new aids?.....

Figure $134-11$. What alert percentage do you think is appropriate to certify a canine for the detection of illicit substances?

Figure $135-12$. How often do you train the canines? 192

Figure 136 - 13. Are you familiar with SWGDOG? 192

Figure 137 - 14. Are you familiar with OSAC? 193

Figure $138-15$. Are your canine teams certified and under what standard? 194 


\section{LIST OF ABBREVIATIONS}

\begin{tabular}{|c|c|}
\hline 2,4-DNT & 2,4-Dinitrotoluene \\
\hline AccuTOF & Accurate Time of Flight Mass Spectrometer \\
\hline $\mathrm{ADC}$ & Analog-to-Digital Converter \\
\hline ANFO & Ammonium Nitrate Fuel Oil \\
\hline ATF & $\begin{array}{l}\text { Bureau of Alcohol, Tobacco, Firearm and } \\
\text { Explosives }\end{array}$ \\
\hline ATR & Attenuated Total Reflectance \\
\hline C-4 & Composition C-4 \\
\hline $\mathrm{CE}$ & Capillary Electrophoresis \\
\hline $\mathrm{CI}$ & Chemical Ionization \\
\hline COMPS & Controlled Odor Mimic Permeation Systems \\
\hline CSA & Controlled Substances Act \\
\hline CT & Computed Tomography \\
\hline DART & Direct Analysis in Real Time \\
\hline DBP & Dibutyl Phthalate \\
\hline DEA & Drug Enforcement Administration \\
\hline DEP & Diethyl Phthalate \\
\hline DIAL & $\begin{array}{l}\text { Differential Absorption Light Detection and } \\
\text { Ranging }\end{array}$ \\
\hline DIRL & $\begin{array}{l}\text { Differential Reflection Light Detection and } \\
\text { Ranging }\end{array}$ \\
\hline $\mathrm{Dm}$ & Molecular Diffusivity \\
\hline
\end{tabular}




\begin{tabular}{cl} 
DPA & Diphenylamine \\
EC & Ethyl Centralite \\
EDS & Explosion Detection Systems \\
EDX & Energy Dispersive X-Ray Analyzer \\
EI & Electron Ionization \\
ETD & Trace Detection Systems \\
FAB & Fast Atom Bombardment \\
FD & Field Desorption \\
FI & Field Ionization \\
FTIR & Fourier Transform Infrared Spectroscopy \\
GC & Gas Chromatography \\
GC-MS & Gas Chromatography - Mass Spectrometry \\
GC-TEA & Gas Chromatography - Thermal Energy Analyzer \\
HMX & High Melting Explosives \\
IC-MS & Ion Chromatography - Mass Spectrometry \\
IED & Improvised Explosive Device \\
IFRI & International Forensic Research Institute \\
IMS & Ion Mobility Spectrometer \\
IR & Infrared Spectroscopy \\
& Liquid Chromatography \\
& Liquid Chromatography - Mass Spectrometry \\
& \\
& \\
LC-MS & xiv \\
\hline
\end{tabular}


Liquid Chromatography - Thermal Energy

LC-TEA Analyzer

LDPE Low Density Polyethylene

LIDAR Light Detection and Ranging

LOX Liquid Oxygen Explosives

LSD Lysergic Acid Diethylamide

MC Methyl Centralite

MCP Microchannel Plate Detector

MDMA Methylenedioxy Methamphetamine

MEMS Microelectromechanical Systems

MIME Metal-Insulator-Metal Ensembles

MMAN Monomethylamine nitrate

MS Mass Spectrometer

NG Nitroglycerin

NMR Nuclear Magnetic Resonance

OSAC Organization of Scientific Area Committee

PCP Phencyclidine

PEG Polyethylene Glycol

PLM Polarizing Light Microscopy

PPE Proper Protective Equipment

QMS Quadrupole Mass Spectrometer

RDX Research Department Explosives 


\begin{tabular}{|c|c|}
\hline RIC & Reconstructed Ion Current \\
\hline RT & Retention Time \\
\hline SAW & Surface Acoustic Wave \\
\hline SCA & Surrogate Continuation Aids \\
\hline SEM-EDS & $\begin{array}{l}\text { Scanning Electron Microscopy - Energy } \\
\text { Dispersive X-Ray Spectrometry }\end{array}$ \\
\hline SLM & Stereo Light Microscopy \\
\hline SWGDOG & $\begin{array}{l}\text { Scientific Working Group on Dog and } \\
\text { Orthagonal Detector Guidelines }\end{array}$ \\
\hline SWGDRUG & $\begin{array}{l}\text { Scientific Working Group for the Analysis of } \\
\text { Seized Drugs }\end{array}$ \\
\hline T/SWGFEX & $\begin{array}{l}\text { Technical/Scientific Working Group for Fire and } \\
\text { Explosion Analysis }\end{array}$ \\
\hline TATP & Triacetone Triperoxide \\
\hline TDC & Time-to-Digital Converters \\
\hline TLC & Thin Layer Chromatography \\
\hline TNA & Trinitroanaline, Picramide \\
\hline TNBA & Trinitrobenzoic acid \\
\hline TNT & Trinitrotoluene \\
\hline TOF & Time-of-Flight \\
\hline TSA & Transportation Security Administration \\
\hline UV & Ultraviolet Spectroscopy \\
\hline VOC & Volatile Organic Compounds \\
\hline XRD & X-Ray Diffraction \\
\hline
\end{tabular}




\section{INTRODUCTION}

Canines are known for their keen sense of smell allowing them to be trained and utilized as detectors for various applications, such as search and rescue missions, location of human remains, and the detection of illicit substances. It is theorized that the canines are alerting to the active odorants that are released from the illicit substances and not the actual controlled substance itself (e.g. methyl benzoate, not cocaine).[1]-[3]

Presently, law enforcement officials utilize trained substance detection canines in routine traffic stops to assess if the vehicle contains any illegal substances. Many believe that this is an infringement on an individual's fourth amendment rights, which has garnered the attention of both the media and the courts. Several questions have been raised with respect to canines alerting to locations where illicit substances were no longer present.

The present study was inspired from an observation made during a canine certification in Miami, Florida, where the handlers would guide the canines through a series of boxes. The second to last box did not contain any odor that would warrant an alert from any of the canines. However, over half of the canines being certified that day made an alert at that box, and it was the only instance where a blank box generated that many unconfirmed alerts. The last box contained a kilo of cocaine, and because of its quantity, it was hypothesized that maybe the odors traveled from that last box to the adjacent box and led to the multiple unconfirmed alerts made by the canines.

Direct Analysis in Real Time - Accurate Time-of-Flight mass spectrometry is an ambient, soft ionization technique that has the capability to sample solids, liquids and gases directly and without the need for sample preparation. The ability to test samples in real 
time, and not having to pre-concentrate the sample via Solid Phase Microextraction (SPME), for instance, makes this instrument ideal for the type of research conducted. Since the instrument can successfully analyze the headspace of volatile compounds when sampled directly into the DART stream, the theory is it could possibly analyze these same volatile organic compounds when held at a distance.

The persistence of these odors on substrates was also considered, to answer questions involving the likelihood of residual odors persisting after briefly being near an illicit substance. The current research looked at using different substrates and various time intervals to try and determine a relationship between the amount of time a substrate is present with volatile organic compounds in an area. Thus, the major purpose of the research was to evaluate the way active odorants transport and persist.

The International Forensic Research Institute (IFRI) at Florida International University (FIU) has a patent on a training-aid kit known as Controlled Odor Mimic Permeation Systems (COMPS), that allows for the controlled release of target volatile organic compounds through a semipermeable membrane. These various training aids are stored together, along with several blank aids, which raises a concern regarding the potential for cross-contamination. Because of the DART's capability for testing in real time, this project explored that possibility by directly sampling the exteriors of their primary and secondary storing containers. If it was determined that there was, in-fact, cross-contamination occurring, it will mean that IFRI might need to reconsider or modify the storage containers, or the manner in which the training aids are stored. 


\section{BACKGROUND INFORMATION}

\subsection{Explosives}

Materials that make up what we now refer to as explosives have been traced back to the $9^{\text {th }}$ century, where Chinese alchemists used to mix potassium nitrate, charcoal and sulfur in certain ratios to form what is now known as black powder. By the mid-eleventh century, these powders would be used as rocket-propelled flame-throwers by packing them in bamboo tubes. Black powder found its way to Europe by the thirteenth century by way of India and Arabia. It remained the only propellant for weapons for centuries until smokeless powders were introduced in the late 1880's.[4]

Explosives have evolved significantly since they were first introduced as black powder and gunpowder. From their original intents for civil applications such as rock blasting and demolition, they have evolved to now include nitrated explosive compounds such as nitroglycerin and nitrocellulose. Today, explosives are classified as either high or low explosives, determined by the means of propagation of the explosive reaction through the material itself. High explosive reactions start with a shock wave that progresses at a speed higher than the speed of sound. Whereas low explosive reactions begin with grain burning at a speed much less than the speed of sound. Explosives can further be subclassified into three major categories: (1) solid, or particulate, propellants, (2) military explosives and (3) commercial explosives.[5]

Propellants include black and smokeless powders. Military explosives include, but are not limited to, picric acid, trinitrotoluene, tetryl, pentaerythritol tetranitrate, plastic explosives, Research Department Explosives (RDX) and High Melting Explosives (HMX). 
Some examples of common commercial explosives are nitroglycerin, dynamite, ammonium nitrate, as well as slurry and emulsion explosives. [5]

Black powder is composed of charcoal, sulfur and potassium nitrate, usually at a ratio of 15:10:75. The manufacturing process involves a mechanical combination of these ingredients which affects the way the powder burns. The first step is known as pulverizing, where the charcoal and sulfur are mixed in a ball mill to allow for the charcoal to be broken into particles and mixed with the sulfur. The first step is the most critical to the quality of the powder, and the quality is improved by the length of time this mixture rotates in the ball mill.[4]

The next step is incorporating, which is the process of rolling or stamping the ingredients together by first reducing the particles to a smaller size, then mixing the ingredients, followed by shearing off the air surface of the particles and finally introducing water. Following the incorporating step is pressing. The pressing step was actually added in the middle of the eighteenth century with the purpose of compacting and consolidating the powder. Pressing adds a few advantages; first, by reducing the space between the particles, it enhances the power of the explosion, secondly, the powder becomes much more stable making it easier to transport, and finally, it allows for more control with respect to the burning speed. Powder density affects the burn rate as the dense powders burn the slowest.[4]

Next is the corning or graining process, which is simply the creation of powder grains. Graining allows for control in the combustion and burn rates. The next step is not used for all powders but still serves a very important purpose. Glazing is done by tumbling 
the powder in barrels to rid the grains of their sharp edges. Finally, the powders go through a drying process, which involves the removal of the water that was added during the incorporation phase, which was added to help dissolve the potassium nitrate.[4]

Even though black powders are used today, and they have been used for centuries, it has its share of pros and cons. The major drawback is that it produces a solid reaction product, such as with the firing of a weapon that produces a dense black cloud. The cloud led to the development of "smokeless" powders. Smokeless powder's early days were also problematic, in that the early iterations of smokeless powders involved a high nitrogen content, but it was deemed too dangerous as it was too prone to accidents. By 1886, a French scientist by the name of Paul Vielle came up with a nitrocellulose material with a lower nitrogen content, that he mixed with ether alcohol, rolled into sheets and cut into small squares and dried. Today, this is considered a "single base" smokeless powder since it only has nitrocellulose. A few years later, Alfred Nobel, came up with a smokeless powder formula he referred to as 'balliste', which he patented. It was a mixture of lownitrated nitrocellulose and nitroglycerin, which is now referred to as a "double-base" powder. Since then, "triple-base" powders were developed by adding nitroguanidine to the mix of nitrocellulose and nitroglycerin.[4], [5]

While black powder is technically the first military explosive, today it includes explosives that are much more powerful than the powder itself. Military explosives are those that are used as the shell filling in artillery rounds as well as for demolition. In the early 1900's, picric acid (2,4,6-trinitrophenol) was the shell filling of choice for the majority of military forces in the world. From picric acid, other explosives such as 
trinitrotoluene (TNT) and tetryl were derived. As shown in the Figure 1 from Cooper and Kurowski, trinitrobenzene is the molecular backbone for this family of explosives [5], [6]. During World War I, TNT became the most abundantly used material for shell-filling and demolition uses. The trend continued until World War II where RDX and HMX became more popular, because of the extensive amount of research done after World War I. Since the first world war, new explosives consist of mixtures of different explosive compounds such as TNT, RDX, HMX, wax, aluminum powder, and plasticizers. For example, the white putty-like deformable explosive material commonly known as Composition C-4 (C4) is made up of RDX and plasticizer at an approximate 9:1 ratio. [5], [7]

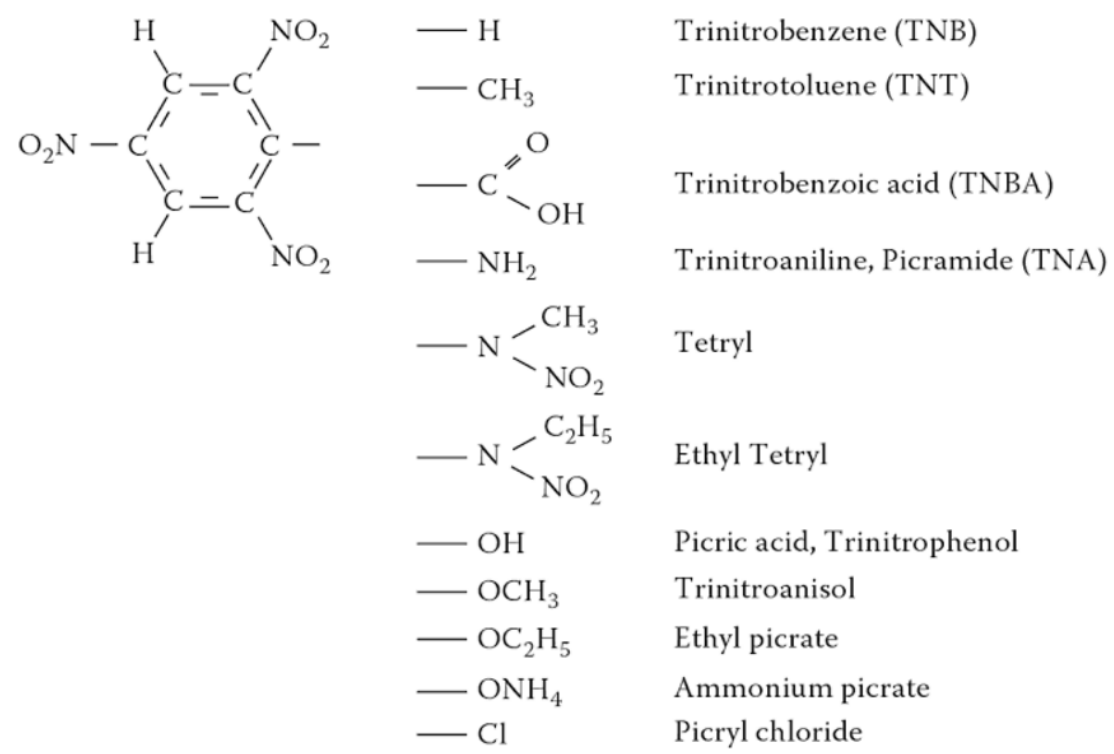

Figure 1 - A family of explosives with molecular backbone of 1,3,5 trinitrobenzene [6].

Commercial explosives, similarly, share a common ancestor with the military explosives in black powder. The earliest reference to its use commercially was in 1627 for mining. To make it less dangerous and more useful, "delay" type materials were invented. 
These started as powder-filled straws, and later would evolve into more sophisticated material. Perhaps the most common commercial explosive known is dynamite. A mixture of nitroglycerin and diatomaceous earth. Alfred Nobel, the namesake of the Nobel Prize, gained notoriety as a result of his association with dynamite. He derived his idea as a result of the difficulty and hazardous nature of shipping nitroglycerin. He discovered that by mixing the liquid with an absorbent material, it would perhaps be easier to handle. Through trial and error, he settled on diatomaceous earth, also known as diatomite, and named the mixture dynamite.[5]

Since Nobel's discovery in 1866, dynamite has been developed using various formulas to serve various purposes, many of which Nobel himself came up with. Later in the 1920's, liquid oxygen explosives (LOX) became a brief competitor to dynamite. LOX is essentially a cloth cartridge with carbon black, that is soaked in liquid oxygen until it is fully saturated. It is then lowered into a borehole, stemmed and subsequently shot. But as a result of the fact that oxygen evaporated rapidly, it limited the number of holes that could be loaded and was therefore seen as a major drawback. Although it was effective and inexpensive, LOX was phased out after 1953, which was the year the United States reached its maximum consumption of approximately 25 million pounds.[5]

While ammonium nitrate has been an ingredient in explosives from early on in the history of explosives, it was mainly used as an oxidizer in mixtures of dynamite. Ammonium nitrate, a small, granular, crystalline particle, is also used in munitions manufacturing and in fertilizer. By the mid-1940's, the production of ammonium nitrate became cheaper from a process called "prilling," which is the process of turning powder 
into pellets. Through prilling, ammonium nitrate became easy to handle and store as a freeflowing, absorbent porous sphere. The formulation of ammonium nitrate is mainly used for fertilizer, whereas the granular variety is still used as a dynamite ingredient. Mixed with carbon black or ground coal proved to be a successful experiment. Around a decade later, it was discovered that fuel oil, instead of solid fuels, were superior. It was at that time that ammonium nitrate fuel oil (ANFO) became the primary explosive used in the appropriate industries.[5]

Although ANFO dominates today's commercial blasting industry, it has several major drawbacks. Firstly, it is not water resistant. Secondly, it has a relatively low density and detonation rate. Because of these limitations, ANFO evolved to become water-based explosives called a "slurry". It consists of ammonium nitrate combined with another oxidizer, water, a gelling agent and a high explosive material. Powdered, grained or flaked forms of aluminum were added frequently for added energy.[5]

An explosion is best described as the resulting exothermic decomposition from heating a reactive material. The rate of the reaction may increase further from the resulting heat and lead to "deflagration;" a self-sustained reaction, or "detonation:" a supersonic but steady rate of reaction. The resulting pressure of an explosion and the magnitude of that same pressure can be estimated by treating the resulting gaseous explosion as ideal gases and can be calculated using the ideal gas law.[5]

$$
P V=n R T
$$

Equation 1 - Where $P$ is pressure, $V$ is volume, $n$ is the moles of gas, $R$ is the gas constant, and $T$ the absolute temperature. 
Low explosives are easily ignited via heat, spark, impact or friction and decompose through the process of deflagration. High explosives, on the other hand, decompose via detonation, initiated through a shockwave. Furthermore, high explosives can be classified as either primary or secondary explosives. Primary high explosives being easily ignitable via friction, shock, heat, or impact while secondary high explosives require initiation via shockwave. Low explosives are more effective when confined as opposed to high explosives, which are effective without the need for confinement. However, containment of a low explosive can turn from deflagration to detonation if the tube containing the explosive is of a sufficient length that allows for the compression waves to accelerate to supersonic speeds.[8], [9]

Both low and high explosives can be initiated via an explosive train. An explosive train can be classified as either low or high depending on the last explosive in the train. A low explosive train would typically have two steps, where a fuse (step one) is used to ignite a low explosive (step two). A high explosive train has two, or more, steps where an initiator, normally a primary high explosive, is used to detonate a secondary explosive.[8], [9]

\subsubsection{Explosives detection}

The detection of explosives ranges from pre-blast to post-blast. The evolution of the technology used to detect explosive material was more rapid after the terrorist attacks on $9 / 11$. Prior to $9 / 11$, airports used metal detection systems, X-ray systems and computed tomography (CT). The metal detection systems were generally for detecting concealed firearms, and while cabinet x-ray machines have the ability to differentiate organic from 
inorganic substances, it could not penetrate substances such as lead, which are high-atomicweight substances. Also, the security officers were not trained to be able to distinguish an improvised explosive device (IED) from a regular non-explosive container.[5]

The technology to detect these explosives did exist, but the tragic events of $9 / 11$ lead to the widespread use of these machines. This included automated explosion detection systems (EDS) and explosive trace detection systems (ETDs). Explosive trace detection systems included the first-generation CT X-ray system, which had the ability to detect explosive materials in checked luggage. An ion mobility spectrometer (IMS) is an example of ETDs which has the ability to detect explosives by looking at the average density of the sample and matching it to a library of known sample spectra.[5]

Since $9 / 11$, there has been an increased amount of terrorist activity worldwide. These extremists started off using both commercial and military explosives, but eventually made the switch to homemade explosives. The reason being that they are much easier to make and gain access to, and many of the ingredients would not raise any red flags when purchased. An example of a common homemade explosive would be triacetone triperoxide (TATP). The recipe of this explosive can be easily found online and made by the oxidation of a commercially available product, acetone.[5]

The tragic truth regarding the progress in detection technology, is that it is reactive, and not proactive. While they are essential in preventing future attacks, they are often developed following an incident, using different chemicals or tools that extremists and radicals come up with. For example, after the Chechen "black widow" suicide bombings in 2004 (referring to the radical female suicide bombers, who were widows of men thought 
to be killed by Russian forces), the Transportation Security Administration (TSA) started to use explosive detection portals that would release puffs of air at a passenger. These air puffs would then be transferred as miniature particles to an IMS detector. Another example was the threat of liquid explosives in 2006, which was the precursor to limiting airline passengers with respect to the amount of liquid they can take on board. This led to the modification of existing x-ray machines to detect liquid explosives. Now, the technology is developed to a point where it could accurately determine the presence of explosives, in addition to weapons, all with a high probability, and low false positive rates.[5]

Explosives can be detected in a physics-based, chemistry-based, and animal-based detection system. Physics-based detection systems refer to the machines previously discussed, which consist of a radiation source and detector, signal and image processing system and the corresponding detection algorithms. Chemistry-based systems involve a chemical reaction platform. Animal-based systems involve an animal detector, such as a canine, trained to alert to the presence of illicit substances. The sensitivity and specificity of the system varies depending on size, location, and circumstance. What all three systems try to achieve is a maximum probability of detection (sensitivity), minimum probability of false positives (specificity), and maximum operational material (throughput). These parameters are closely tied to one another as shown in the ternary diagram in Figure 2. As Bevridge explains in the book "Forensic Investigation of Explosions," when looking at points $\mathrm{A}$ and $\mathrm{B}$, which have similar specificity or false-alarm rate, and increasing the throughput between the two points, it causes a decrease in sensitivity. Whereas when points $\mathrm{A}$ and $\mathrm{C}$ are considered, which have the same sensitivity, a decrease in throughput is required to increase the specificity, or decrease false alarms. When considering points $\mathrm{C}$ 
and $\mathrm{D}$, which are equal in terms of throughput, by increasing sensitivity from point $\mathrm{D}$ to $\mathrm{C}$, it causes specificity to decrease or the false alarm rate to increase.[5]

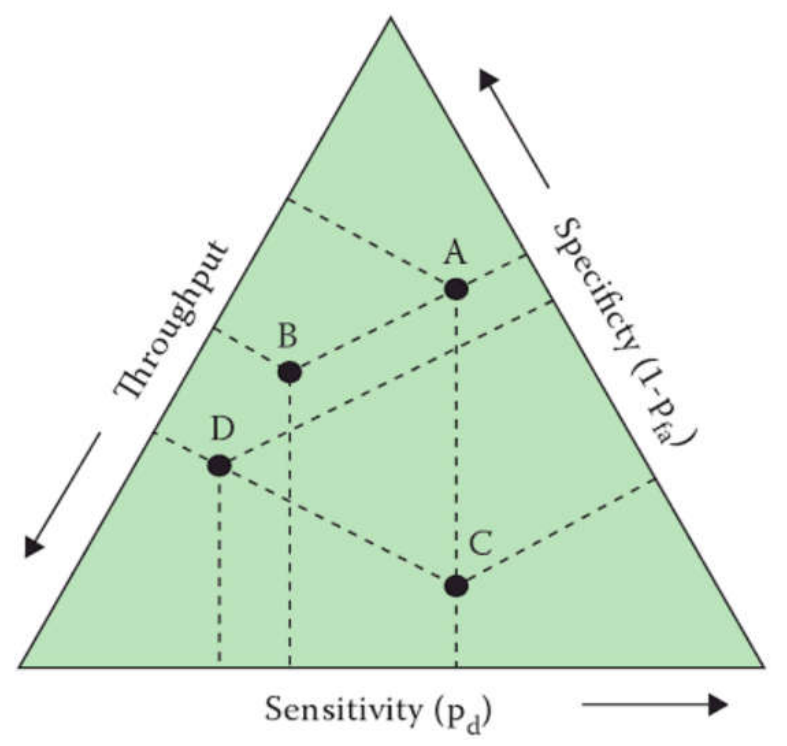

Figure 2 - The three most important parameters for explosive detection systems [5].

Logically, the majority of research done regarding explosives from a law enforcement aspect involves detection prior to its combustion (pre-blast). What is additionally important, is the ability to detect and identify explosives after combustion (post-blast) that are encountered at the crime scene. After the evidence is properly documented and collected, it is sent to the appropriate forensic laboratory. In most jurisdictions, explosive evidence is analyzed in the trace evidence sections of forensic science laboratories or departments. While standards of procedures may differ slightly from laboratory to laboratory, the underlying principals and questions remains the same and are for the most part derived from recommendations suggested by the Technical/Scientific Working Group for Fire and Explosion Analysis (T/SWGFEX).[10] 
When a forensic scientist begins their analysis, they first start by performing a detailed physical examination, which in turn will help determine which subsequent analytical procedures to perform. By examining the material, it can be determined whether or not any unconsumed low explosive materials are present. For post-blast explosive residues, T/SWGFEX has a list of techniques divided into four categories as shown in Table 1. Categories 1 and 2 provide significant and limited (respectively) structural and/or elemental information. Category 3 involves techniques that provide a high degree of selectivity. Techniques under Category 4 are considered useful but do not fit in the other three categories.[10]

Table 1 - Techniques recommended for post-blast forensic analysis as suggested by T/SWGFEX[10].

\begin{tabular}{|c|c|c|}
\hline Categories 1 and 2 & Category 3 & Category 4 \\
\hline Infrared Spectroscopy (IR) & Gas Chromatography (GC) & Flame Test \\
\hline $\begin{array}{c}\text { Gas Chromatography-Mass } \\
\text { Spectrometry (GC-MS) }\end{array}$ & $\begin{array}{c}\text { Gas Chromatography Thermal Energy } \\
\text { Analyzer (GC-TEA or EGIS) }\end{array}$ & Spot Test \\
\hline $\begin{array}{c}\text { Energy Dispersive X-Ray } \\
\text { Analyzer (EDX) }\end{array}$ & $\begin{array}{c}\text { Melting } \\
\text { Point }\end{array}$ \\
\hline Raman Spectroscopy & $\begin{array}{c}\text { Liquid Chromatography (LC) } \\
\text { Energy Analyzer (LC-TEA) }\end{array}$ & \\
\cline { 1 - 2 } X-Ray Diffraction (XRD) & Ion Chromatography (IC) & \\
\cline { 1 - 2 } $\begin{array}{c}\text { Liquid Chromatography-Mass } \\
\text { Spectrometry (LC-MS) }\end{array}$ & Capillary Electrophoresis (CE) & \\
\cline { 1 - 2 } $\begin{array}{c}\text { Ion chromatography-Mass } \\
\text { Spectrometry (IC-MS) }\end{array}$ & Thin Layer Chromatography (TLC) & \\
\cline { 1 - 2 } X-Ray Fluorescence (XFR) & Ion Mobility Spectrometry (IMS) & \\
\cline { 1 - 2 } & Polarizing Light Microscopy (PLM) & \\
\cline { 1 - 2 } & Stereo Light Microscopy (SLM) & \\
\hline
\end{tabular}

If any unconsumed black powder is present, the analyst would note the physical appearance of the powder, then perform an ignition test, followed by a microchemical test. The ignition test is done to determine how that powder reacts when ignited. For example, 
smokeless powders have a distinct smell that is a result of the sulfur burning. The microchemical tests are another way to screen for explosive compounds by determining which class of compounds are present, such as sulfates and nitrates, that can help narrow down which subsequent confirmatory tests need to be performed.[11]

Following the ignition and microchemical tests, if the powders are presumed to be a smokeless powder, they are analyzed using a Fourier Transform Infrared Spectroscopy (FTIR) instrument. The sample can be prepared in one of four ways:

(1) - Grinding the sample with IR grade potassium bromide $(\mathrm{KBr})$ to create a pellet.

(2) - Adding sample between a micro-compression cell with diamond windows, and hand tighten to compress.

(3) - Using the Attenuated Total Reflectance (ATR) accessory, which involved adding the sample onto the diamond portion of the crystal.

(4) - Adding acetone to the $\mathrm{KBr}$ pellet and testing in transmittance mode.

If it is confirmed to be a smokeless powder, the next step is to determine whether it is a single or double based powder. The identification is accomplished by extracting the powder in a solution of methylene chloride or acetone and analyzed using a Gas Chromatography - Mass Spectrometer (GC-MS). The GC-MS step is done to determine the presence or absence of nitroglycerin. The absence of nitroglycerin would mean it is a single base powder, whereas the presence of nitroglycerin means that it is a double base powder.[11]

If the powder is determined to be a black powder following the screening test, the next step would be to confirm that result by using an X-Ray Diffraction (XRD) instrument. The XRD helps determine the composition of a sample based on its crystalline structure. 
If the powder is instead determined to be a flash powder, then an extra step is added following XRD analysis, which is done using a Scanning Electron Microscopy - Energy Dispersive X-Ray Spectrometry (SEM-EDS). If the powder was determined to be pyrotechnic powder, then the procedure would involve the same steps done when analyzing flash powders, with the addition of Ion Chromatography (IC).[11]

The presence of unconsumed powder is ideal, as the analysis is rather straight forward. If, however, no unconsumed powder was identified, then the sample will go through various extraction techniques, unless a large amount of consumed powder residue is present. Firstly, methanol extracts monomethylamine nitrate (MMAN). An ether extraction follows to extract nitroglycerin, which helps with identifying double base smokeless powders, as well as dynamite. Next, acetone is used to extract most organic explosives, including nitrocellulose. Water is then used to extract the inorganic explosives. Following extraction, the screening and confirmatory steps are identical to the steps used with unconsumed powders.[11]

If intact high explosives are submitted as evidence to the laboratory, the examiner must use their best judgement on how best to proceed. Following visual examinations, either macroscopic or microscopic, the examiner can determine the next steps depending on the amount of sample present. If it is determined that there is a limited amount of sample, then nondestructive tests become a priority.[11]

The forensic analysis of intact C-4, for example, was developed in 1986 by Raymond Keto. Keto sought to develop a sensitive and definitive method to examine C-4. The C-4 analysis involves simple solvent extractions and filtrations to separate the RDX 
from the plasticizers, rubber binder and motor oils. His extraction technique is still used for the analysis of C-4 three decades later. Following extraction, RDX can then be identified using FTIR.[7]

\subsection{Narcotics}

Legal statutes for drug possession vary among the local, state, and federal jurisdictions. The Controlled Substances Act (CSA) of the 1970's is considered to be the legal foundation for drug enforcement in the nation on the federal level [12]. It mainly regulates the manufacture and distribution of drugs. It also created the scheduling categories that are still used today. The scheduling is differentiated according to a few factors; (1) the narcotics potential for abuse, (2) potential for psychological or physical dependence, and (3) acceptable medical use in the United States.

The CSA allows for newly discovered narcotics to be controlled, and thus added to a schedule, while also allowing for 'decontrolling' a controlled substance, which would remove the substance from their schedule. It also allows for 'rescheduling', moving a controlled substance from one schedule to another. There are five federal-level schedules (I-V) that vary according to the aforementioned factors.

Schedule I narcotics include, but are not limited to, heroin, MDMA, LSD, cathine and marijuana. They are in this schedule because of their high potential for abuse, high potential for dependence, and lack of acceptance in the medical field in the United States. Penalties for possession of Schedule I narcotics ranges from a minimum of 5 years for first time offenders to life imprisonment for repeat offenders, with fines between $\$ 1-20$ million dollars for an individual. [13], [14] 
Of all the Schedule I drugs, only heroin, LSD and fentanyl analogues differ in their penalties depending on the amount seized. For example, if an individual is caught in possession of 100-999 grams of heroin, 1-9 grams of LSD, or 10-99 grams of fentanyl analogues, then the penalty is no less than 5 years and no more than 40 years if it was the first offense (with a fine not exceeding $\$ 5,000.000$ for an individual), no less than 10 years and not more than life if it were a second offense (with fines up to $\$ 8,000,000$ for an individual). For quantities greater than those aforementioned, then it is no less than 10 years and not more than life for first time offenders, with fines up to a maximum of $\$ 10,000,000$ for individuals. For second offenses, it is no less than 20 years and not more than life, with a fine not exceeding $\$ 20,000,000$. If an individual has more than 2 prior offenses the penalty is life imprisonment with a fine of not more than $\$ 20,000,000$. [13], [14]

Schedule II narcotics include, but are not limited to, cocaine, amphetamine, phencyclidine (PCP), fast acting barbiturates, morphine and other opiates. Although these substances have a high potential for abuse and high potential for physical and psychological dependence, there is an accepted medical use currently for these narcotics.[13]

Similarly, there are a few Schedule II narcotics that have different penalties based on the amounts seized. For 500-4999 grams of cocaine, 28-279 grams of cocaine base, 50499 grams of methamphetamine (or 5-49 grams if pure), or 100-999 grams of PCP (or 1099 grams if pure), the penalties are identical to that of heroin, LSD and fentanyl analogues in their lower ranges. Anything above those ranges for cocaine, methamphetamine and PCP, then the penalties are identical to the higher ranges for heroin, LSD and fentanyl 
analogues. The remaining Schedule II narcotics have the same penalties as the remaining Schedule I narcotics. [14]

Schedule III controlled substances, such as anabolic steroids and codeine, have less potential for abuse when compared to Schedule I \& II drugs, and lower potential for psychological or physical dependence than Schedule I \& II drugs, while also having currently accepted medical usage in the United States. Possession of all Schedule III drugs results in the same penalty; no more than 10 years in prison with a maximum fine of $\$ 500,000$ for first offenders. For second offenders, it is no more than 20 years in prison with a maximum fine of $\$ 1,000,000$. [13], [14]

Schedule IV drugs, such as phenobarbital, diazepam (Valium), chloridiazepoxide (Librium), and Meprobamate (Miltown), have lower potential of abuse and limited dependence relative to Schedule III drugs. The penalties for possession of Schedule IV drugs for first offenses is not more than 5 years in prison, and fines up to $\$ 250,000$. Whereas it is no more than 10 years in prison, and fines up to $\$ 500,000$ for second offenses. [13], [14]

Schedule V drugs, such as low concentrations of codeine, typically found in inhalers or cough medicine, have a much lower potential for abuse and a lot less potential for psychological and physical dependence than the rest of the controlled substances. Both Schedule IV and V are obviously accepted and used in the medical field currently. Penalties for possession of Schedule V drugs are the least harsh, with no more than 1 year for first time offenses with a maximum fine of $\$ 100,000$. Not more than 4 years in prison and a 
maximum fine of $\$ 200,000$ for second time offenses. A table summarizing which drugs belong to which Schedule is shown below in Table 2.[13], [14]

Table 2 - Summary of common controlled substances and their DEA schedule and corresponding penalties [14].

\begin{tabular}{|c|c|c|}
\hline Schedule & Examples & Penalties \\
\hline \multirow{4}{*}{ I } & Heroin & \multirow{4}{*}{$\begin{array}{l}\text { *First Offense: Not more than } 20 \text { years } \\
\text { Not more than } \$ 1 \text { million } \\
\text { *Second Offense: Not more than } 30 \text { years } \\
\text { Not more than } \$ 2 \text { million }\end{array}$} \\
\hline & LSD & \\
\hline & Marijuana & \\
\hline & MDMA & \\
\hline \multirow{4}{*}{ II } & Cocaine & \multirow{4}{*}{$\begin{array}{l}\text { *First Offense: Not more than } 20 \text { years } \\
\text { Not more than } \$ 1 \text { million } \\
\text { *Second Offense: Not more than } 30 \text { years } \\
\text { Not more than } \$ 2 \text { million }\end{array}$} \\
\hline & Morphine and other opiates & \\
\hline & Phencyclidine (PCP) & \\
\hline & Amphetamines & \\
\hline \multirow{4}{*}{ III } & Barbiturates & \multirow{4}{*}{$\begin{array}{l}\text { First Offense: Not more than } 10 \text { years } \\
\text { Not more than } \$ 500,000 \\
\text { Second Offense: Not more than } 20 \text { years } \\
\text { Not more than } \$ 1 \text { million }\end{array}$} \\
\hline & Anabolic Steroids & \\
\hline & Buprenorphine & \\
\hline & Empirin & \\
\hline \multirow{4}{*}{ IV } & Rohypnol & \multirow{4}{*}{$\begin{array}{c}\text { First Offense: Not more } 5 \text { years } \\
\text { Not more than } \$ 250,000 \\
\text { Second Offense: Not more than } 10 \text { years } \\
\text { Not more than } \$ 500,000\end{array}$} \\
\hline & Phenobarbital & \\
\hline & Valium & \\
\hline & Librium & \\
\hline \multirow{4}{*}{ V } & Codeine preparations $<200 \mathrm{mg}$ & \multirow{4}{*}{$\begin{array}{l}\text { First Offense: Not more than } 1 \text { year } \\
\text { Not more than } \$ 100,000 \\
\text { Second Offense: Not more than } 4 \text { years } \\
\text { Not more than } \$ 200,000\end{array}$} \\
\hline & Motofen & \\
\hline & Cophene-S & \\
\hline & Lomotil & \\
\hline
\end{tabular}

* With Exceptions

Despite the strict laws concerning narcotics possession and/or distribution, and the efforts made by various federal, state and local officials, the number of yearly arrests seems to remain constant. Figure 3 shows the number of domestic drug-related arrests made between 2005 and 2015 by the DEA, where the number of arrests go up or down with no particular trend. With a low of 28,607 arrests in 2008 and a high of 32,530 in 2011, the number of yearly arrests by the DEA are rather consistent.[15] 


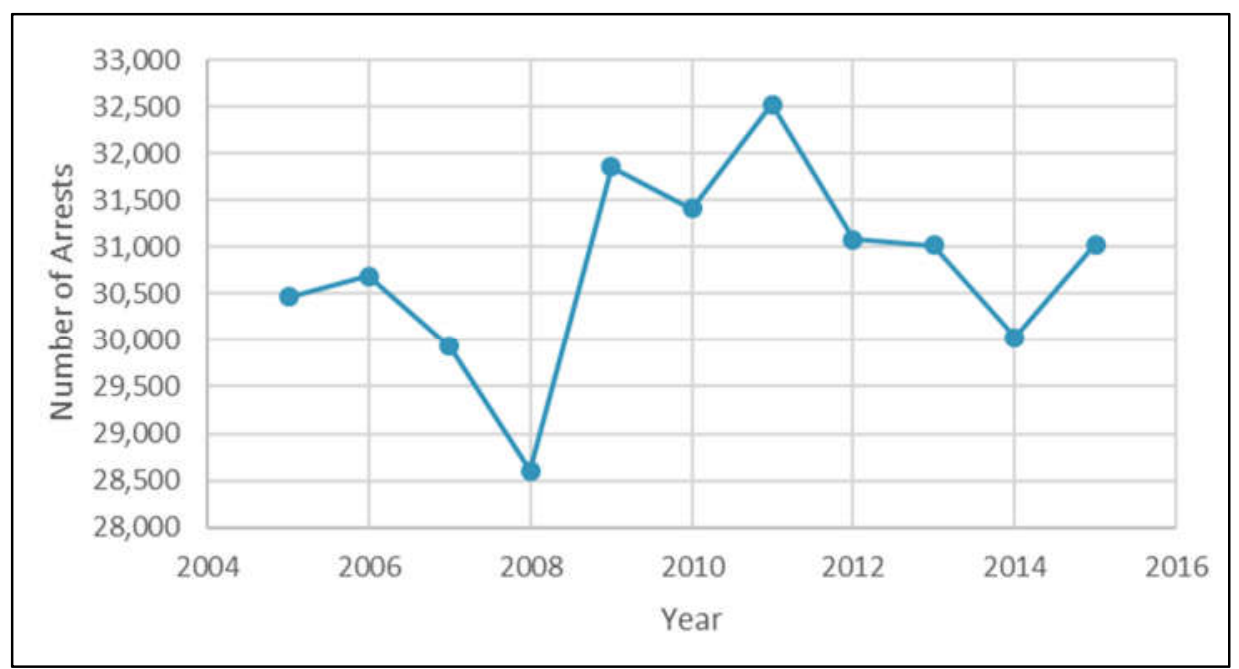

Figure 3 - Number of drug related domestic arrests from 2005-2015 [15].

There are, however, trends with respect to the yearly amounts of narcotics seized. The published numbers include the number of kilograms of cocaine, heroin, methamphetamine and marijuana seized up until the year 2013. The 2014 data were not complete and were therefore omitted. As can be seen in Table 3, marijuana seizures account for the majority of the activity done by the DEA. Cocaine is the second highest, and when looking at a plot versus marijuana it shows that the amount being seized yearly for both is trending downwards (Figure 4). 
Table 3 - DEA drug seizure amounts from 2003-2013 [15].

\begin{tabular}{|c|c|c|c|c|}
\hline $\begin{array}{c}\text { Calendar } \\
\text { Year }\end{array}$ & $\begin{array}{c}\text { Cocaine } \\
\text { (kgs) }\end{array}$ & $\begin{array}{c}\text { Heroin } \\
\text { (kgs) }\end{array}$ & $\begin{array}{c}\text { Marijuana } \\
\text { (kgs) }\end{array}$ & $\begin{array}{c}\text { Methamphetamine } \\
\text { (kgs) }\end{array}$ \\
\hline 2003 & 73,720 & 788 & 254,242 & 1,680 \\
\hline 2004 & 117,844 & 669 & 266,088 & 1,656 \\
\hline 2005 & 118,128 & 622 & 283,382 & 2,161 \\
\hline 2006 & 71,604 & 816 & 328,275 & 1,804 \\
\hline 2007 & 98,065 & 623 & 360,708 & 1,112 \\
\hline 2008 & 50,461 & 605 & 662,137 & 1,518 \\
\hline 2009 & 50,705 & 622 & 671,650 & 2,129 \\
\hline 2010 & 30,061 & 713 & 725,862 & 2,224 \\
\hline 2011 & 32,151 & 1,077 & 575,972 & 2,561 \\
\hline 2012 & 36,736 & 1,010 & 388,064 & 4,813 \\
\hline 2013 & 24,103 & 1,044 & 270,823 & 4,227 \\
\hline
\end{tabular}

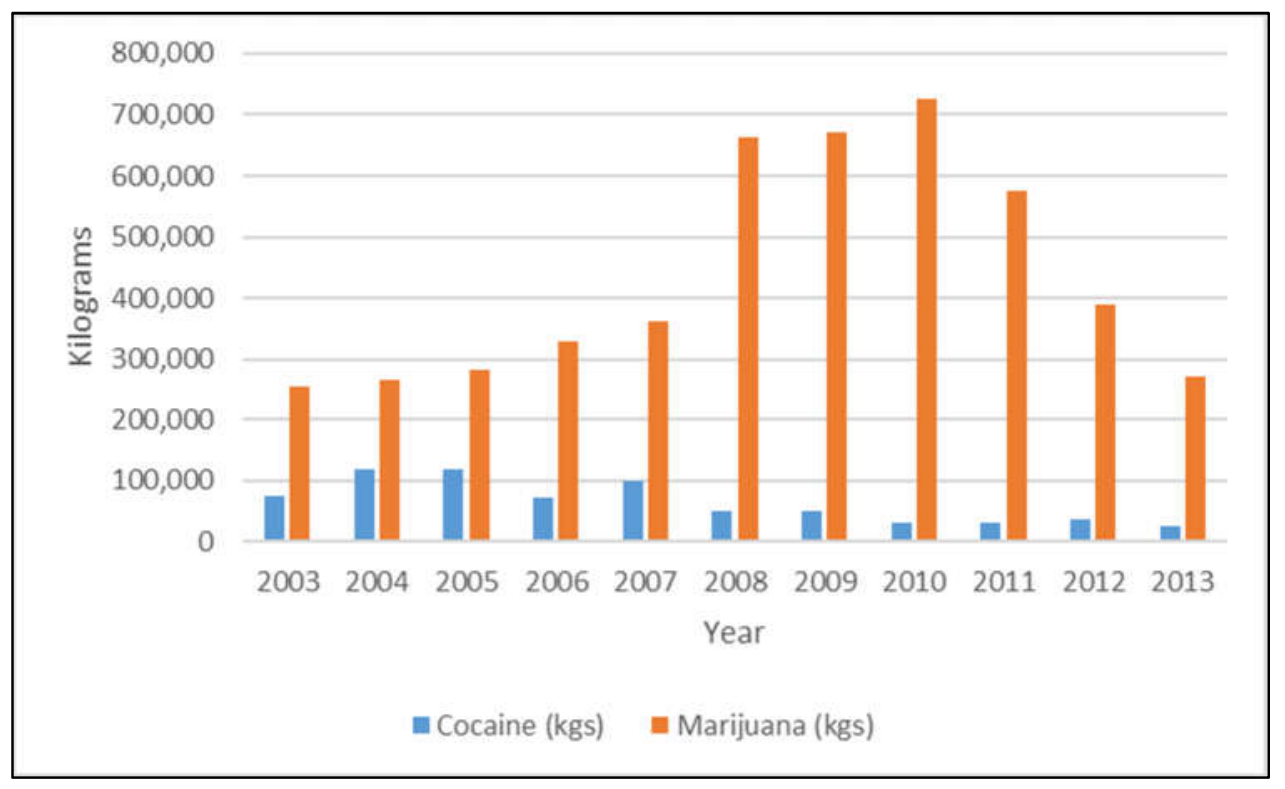

Figure 4 - Illustrating the trend between yearly drug seizures of marijuana versus cocaine between 2003-2013 [15].

Whereas the trend for heroin and methamphetamine is in the opposite direction as illustrated in Figure 5. While the amount of marijuana and cocaine seized has been cut in almost half in the last five years of this data set, the amount of heroin and methamphetamine has practically doubled. Despite that, marijuana and cocaine, by an 
overwhelming margin, remain the two most frequently encountered controlled substances by law enforcement officials and forensic laboratory technicians. The data is suggests that if a forensic scientist would normally encounter a few cases of heroin and methamphetamine per week in 2009 , then they would see double that amount on a weekly basis today.[15]

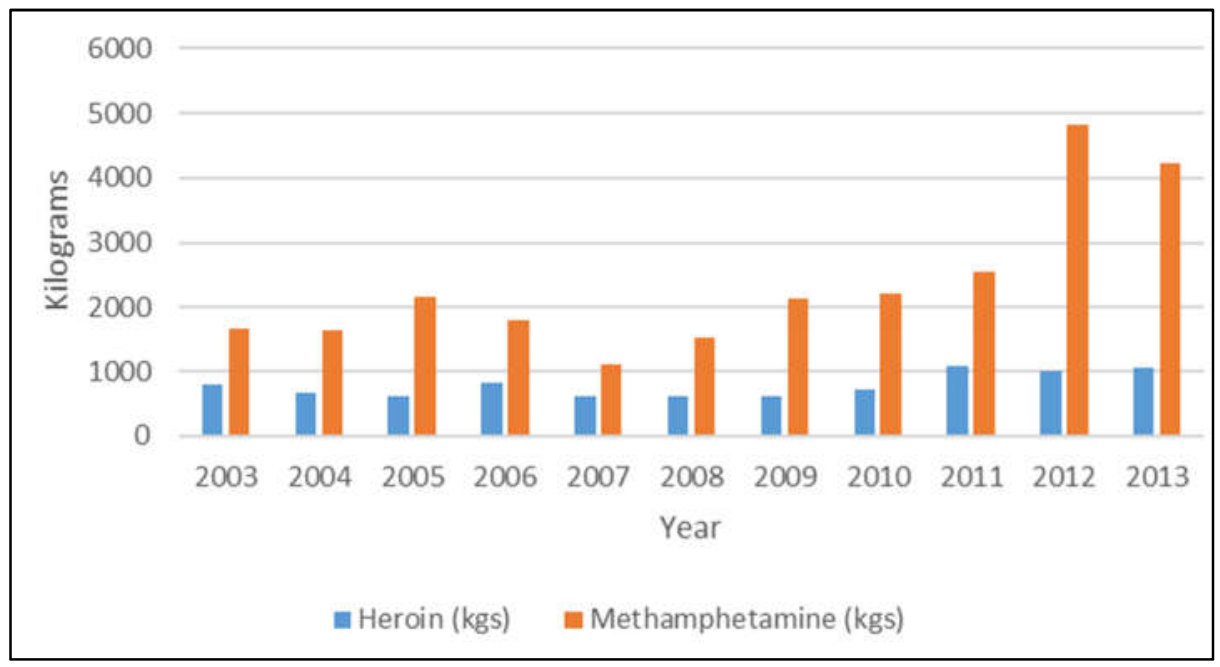

Figure 5 - Illustrating the trend between yearly drug seizures of heroin versus methamphetamine between 2003-2013 [15].

In the laboratory, the analytical scheme to be followed is determined by the physical nature of the evidence. The Scientific Working Group for the Analysis of Seized Drugs (SWGDRUG) has a manual with recommendations for how best to analyze controlled substances depending on its various forms. If it is in a powder or solid form, but not a tablet or capsule, the analysis is conducted in a particular sequence. If it is a residue, it may require an additional rinsing step if it is not physically removable, before the screening and confirmatory steps. Tablets and capsules differ in their screening because they often have pharmaceutical identifiers, and so it requires a different set of sequences for analysis. [16], [17] 
In the SWGDRUG manual, it is required that the analyst uses multiple uncorrelated techniques. It published a table (Table 4) showing the three major categories of analytical techniques. It recommends that when a validated Category A technique is used, then only one other technique from either Categories $\mathrm{A}, \mathrm{B}$ or $\mathrm{C}$ need to be used. Whereas if a Category A technique is not used, then a minimum of three techniques must be used, where at least two of three techniques from Category B, as is usually the case for the analysis of plant material.[17]

Table 4 - The major categories of analytical techniques as suggested by SWGDRUG [17].

\begin{tabular}{|c|c|c|}
\hline Category A & Category B & Category C \\
\hline Infrared Spectroscopy & Capillary Electrophoresis (CE) & Color Tests \\
\hline Mass Spectrometry & Gas Chromatography (GC) & $\begin{array}{l}\text { Fluorescence } \\
\text { Spectroscopy }\end{array}$ \\
\hline $\begin{array}{l}\text { Nuclear Magnetic } \\
\text { Resonance (NMR) }\end{array}$ & Ion Mobility Spectrometry (IMS) & Immunoassay \\
\hline Raman Spectroscopy & Liquid Chromatography (LC) & Melting Point \\
\hline \multirow[t]{4}{*}{$\begin{array}{l}\text { X-ray Diffractometry } \\
(\mathrm{XRD})\end{array}$} & Microcrystalline Tests & $\begin{array}{l}\text { Ultraviolet (UV) } \\
\text { Spectroscopy }\end{array}$ \\
\hline & Pharmaceutical Identifiers & \\
\hline & $\begin{array}{c}\text { Thin Layer Chromatography } \\
\text { (TLC) }\end{array}$ & \\
\hline & $\begin{array}{l}\text { Cannabis only: } \\
\text { Macroscopic Examination } \\
\text { Microscopic Examination }\end{array}$ & \\
\hline
\end{tabular}

When an examiner begins their analysis and the unknown is in powder form, the first thing they are required to do is to weigh it. They would then sample the unknown and perform color, or spot, tests. This is a quick screening technique that is performed on clean porcelain or disposable plastic spot plates with various reagents. An example for how an examiner would first start with a white powder would be to first use cobalt thiocyanate as a reagent. The formation of a blue precipitate will indicate that the sample could potentially 
be cocaine base, PCP or even a barbiturate. A table of the various color tests used and their results is shown in Table 5. [16], [18]

Table 5 - Results of the most common spot tests and their expected color change [18].

\begin{tabular}{|c|c|c|}
\hline Test & Drug Analyte & Color Change \\
\hline Cobalt Thiocyanate & Cocaine & Strong greenish blue \\
\hline Dille-Koppanyi & Barbitals & Light purple \\
\hline Duquenois-Levine & THC & Purple \\
\hline \multirow{4}{*}{ Mandelin } & Asprin, acetometaphen & Olive green \\
\hline & Cocaine & Deep orange-yellow \\
\hline & Methamphetamine & Dark yellowish-green \\
\hline & Opium & Dark Brown \\
\hline \multirow{3}{*}{ Marquis } & Methamphetamine & Dark reddish-orange \\
\hline & LSD & Olive-black \\
\hline & Mescaline & Strong orange \\
\hline \multirow{3}{*}{ Nitric Acid } & LSD & Strong brown \\
\hline & Opium & Dark orange yellow \\
\hline & Oxycodone & Brilliant yellow \\
\hline p-DMAB & LSD & Deep purple \\
\hline Ferric Chloride & Morphine & Dark green \\
\hline \multirow{2}{*}{ Froehde } & MDA (amphetamine) & Greenish black \\
\hline & Oxycodone & Strong yellow \\
\hline \multirow{2}{*}{ Mecke } & MDA & Dark bluish-green \\
\hline & Mescaline & Moderate olive \\
\hline Zwikker & Barbitals & Light purple \\
\hline Simon's (Nitroprusside) & Methamphetamines & Dark blue \\
\hline
\end{tabular}

The next screening procedure is commonly done using thin layer chromatography (TLC). Thin layer chromatography is used to separate components of an unknown mixture 
against known drug standards. Separation is dependent upon the $\mathrm{pH}$ or the polarity of the sample, solvent bath, and stationary phase used on the plate. If an unknown sample contains cocaine, for example, then there will be a visual spot on the plate that lines up with known drug standard after enhancement. An example of a plate can be seen below in Figure 6.[16], [19], [20]

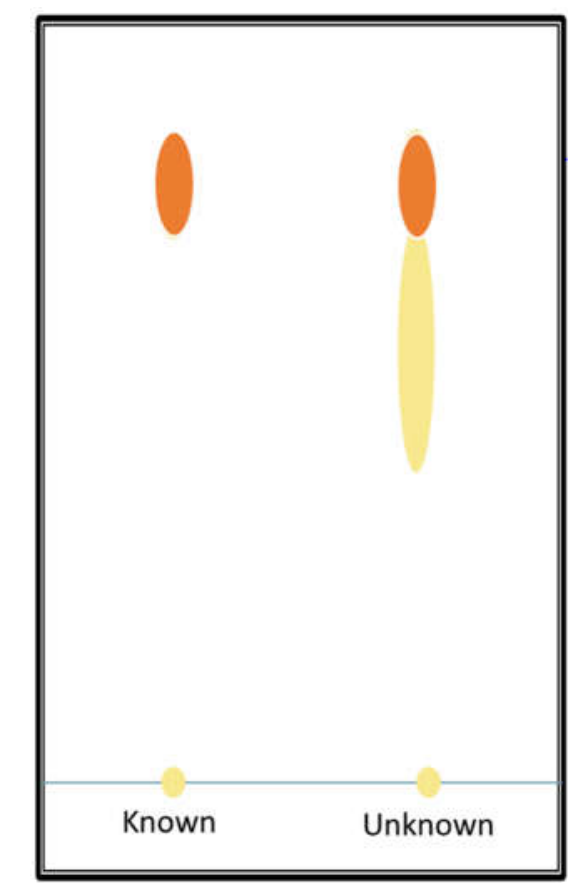

Figure 6 - Example of a TLC plate.

Finally, the results are then confirmed using GC/MS, LC/MS or FTIR and reported. If the evidence being analyzed is in residue form, the first step is to try to physically remove the residue. If it is easily removed, then the analytical scheme is the same as samples in powder form. If not, the residue would be rinsed and the color test would be skipped in lieu of TLC. The remaining steps are identical.[16] 


\subsection{Detection of Illicit Substances Outside of the Laboratory}

What has been discussed thus far involves illicit substances that have been collected as evidence and sent to the laboratory for analysis. Figure 7 illustrates the existing techniques and those that are under development for the identification of trace amounts of volatile organic compounds (VOC)[21]. These compounds originate from various forensic specimens such as drugs and explosives, as well as human scent[22]. By being able to identify or even locate these illicit substances, investigators can potentially prevent irreparable harm to the public. The use of biological detectors greatly enhances the ability for law enforcement officials to do due their numerous advantages.[22]

Figure 7 also shows the various arrays that can be used independently involve sensor beads, polymeric thin films, nanocluster metal-insulator-metal ensembles (MIME) and fluorescent polymers, whereas surface acoustic wave (SAW) detectors and microelectromechanical systems (MEMS) can be used in conjunction with GC detectors[21]. It also shows several optical detection methods used for imaging that are under development such as laser, light detection and ranging (LIDAR), including differential absorption LIDAR (DIAL) as well as differential reflection LIDAR (DIRL).[21] 


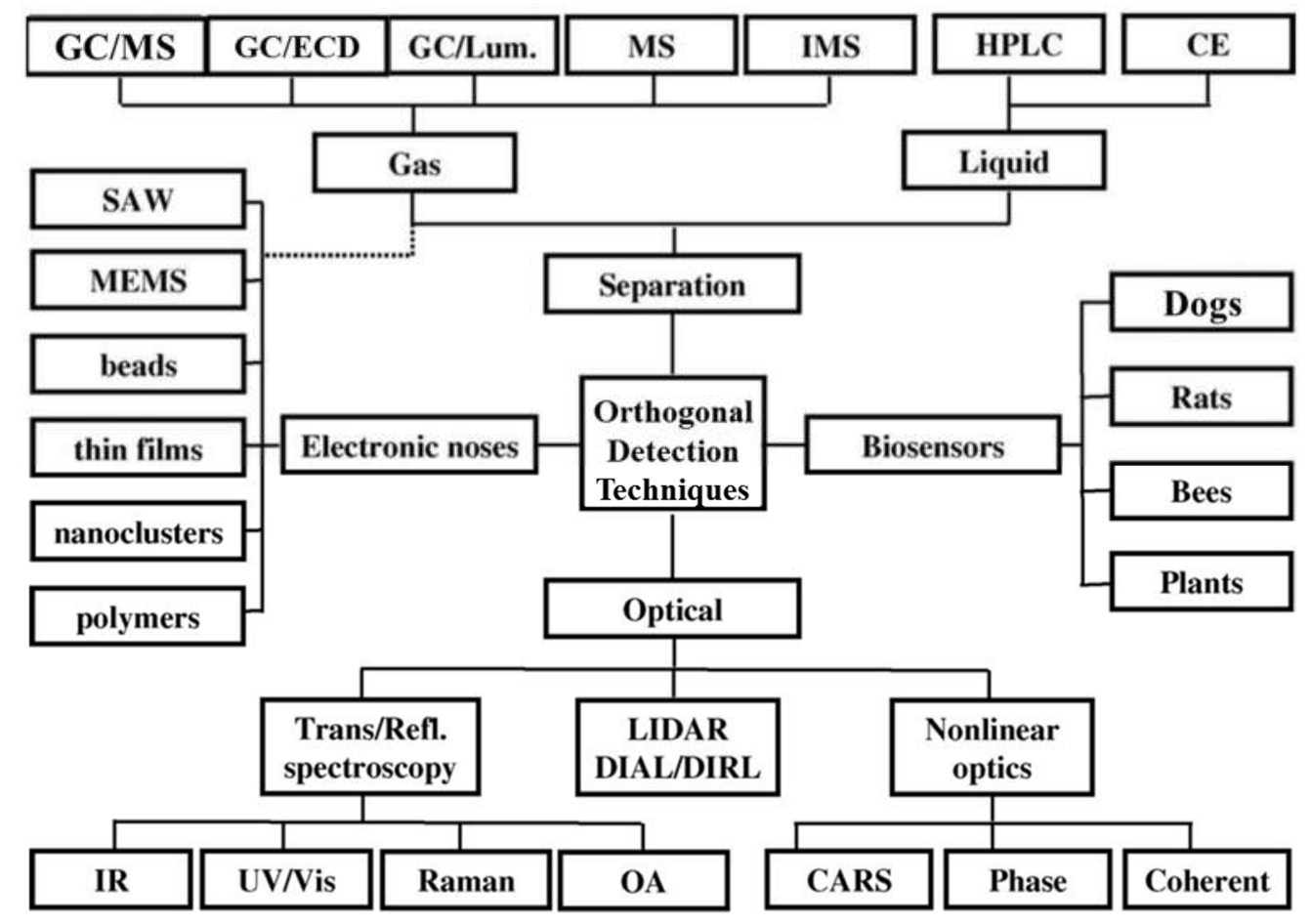

Figure 7 - A chart illustrating the several detection methods for trace explosive detection currently available and also under development [21].

In addition to the various separation and optical techniques, there are several detection methods that involve the use of biological sensors. The Belgian research organization (APOPO), found that rats could be trained to detect landmines[23]. Bees have also been able to demonstrate their capability of detecting explosives at concentrations lower than even modern instruments can detect[24]. Arsea Biodetection in Denmark was able to genetically modify a cress crop so that its leaves would change colors when in close proximity to landmines[25].

Like most methods, there are obvious disadvantages which make these biosensors less favorable to use. While rats are small and relatively inexpensive, their training and retrieval make them problematic[23]. Bees, on the other hand, can be trained quickly and 
their retrieval to the beehive is not an issue[24]. However, bees do not fly at night, nor do they fly during heavy rainfall or cold weather[24]. Lastly, the modified cress crop is useful when in contact with $\mathrm{NO}_{2}$ in the soil from leaking landmines[25]. If those landmines are properly sealed to prevent seepage, then the crops will not be able to detect any $\mathrm{NO}_{2}$ in the soil and therefore will not change colors.[21], [25]

\subsubsection{Canines \& Forensic Science}

While the sensitivity of modern instrumentation has improved dramatically over the past decades, it still does not rival the speed, versatility, ruggedness and relatively low cost provided by canines[22]. The use of canines (Canis familiaris) in law enforcement goes back to the $14^{\text {th }}$ century, in St. Malo, France, where they were used to guard naval installations and docks [26]. Later in the $19^{\text {th }}$ century, Sir Charles Warren, Commissioner of the Metropolitan Police in London, famously used bloodhounds to search for Jack the Ripper [22], [27].

A little over a decade later in 1899 , the Belgian police force in Ghent began to formally train dogs for police work. Their popularity spread rapidly in Europe and by 1910, Germany had trained canines in over 600 of their largest cities. In 1938, two specially trained Labrador retrievers used to accompany officers on patrol in London. It was not until the seventies that canines were used in law enforcement in the United States.[28]

Today, canines are considered an integral part of police departments and, in many instances, are issued their own badges. Of the hundreds of dog breeds, the most popular breed used with law enforcement is the German Shepherd, because of their intelligence, strength, obedience and ability to be taught and trained. Other popular breeds in law 
enforcement include the Labrador Retriever, Belgian Malinois, and the Dutch Shepherd.[28]

\subsubsection{The Anatomy of the Canine Nose and its Olfactory System}

The remarkable olfactory abilities of canines are a result of millions of years of natural selection and evolution. The canine nose has five other simultaneous physiological functions in addition to odor detection; respiration, water balance, recycling tears, temperature regulation and vocalization. The biological system responsible for these physiological functions is referred to as the olfactory system.[29]

The olfactory system is made up of the olfactory epithelium, olfactory nerves, olfactory receptors, olfactory bulbs, mitral cells and glomeruli. The olfactory epithelium, recessed in the rear of the canine's nose, is lined with hundreds of millions of sensory neurons. To put the olfactory acuity of canines in perspective, their ability to detect odors at a concentration of 1-2 parts per trillion (ppt) is approximately 10,000-100,000 times that of humans. Often times the olfactory acuity is attributed to the olfactory organ size or neuronal density, but it can also be attributed to the anatomical structure of the nasal cavity and the transport of the odor to receptors on the cilia of the olfactory epithelium via sniffing (Figure 8).[30]-[33] 


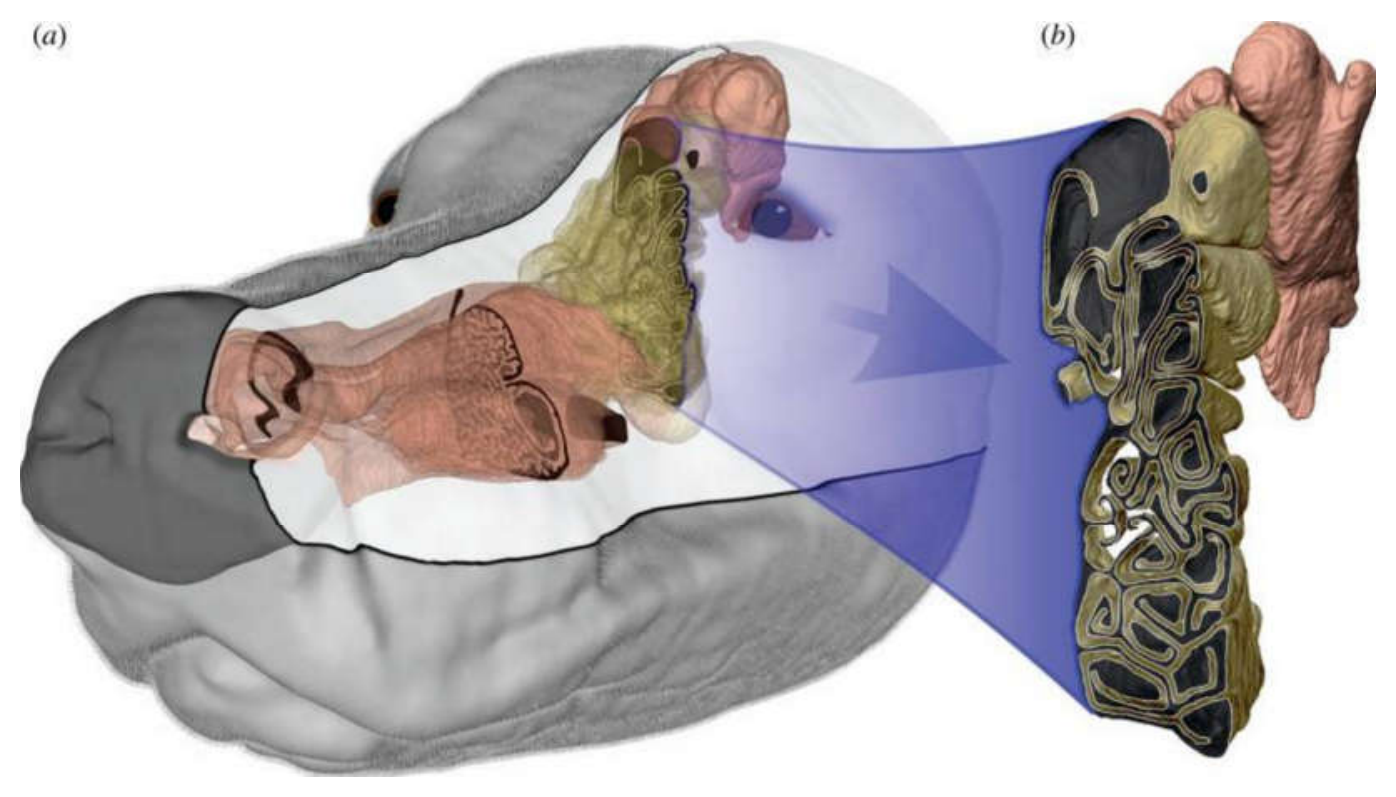

Figure 8 - (a) Is a 3D model of the left side of the canine nasal airway and (b) shows the olfactory recess in the rear of the cavity [32].

Once in the nasal cavity, odorants are absorbed into the mucus layer and are then passed through the cell membrane via transfer and receptor proteins. As the odorants are absorbed into the cells, they then encounter the tissue that houses the olfactory receptors (olfactory epithelium). The absorption into the cells creates a nerve impulse that is subsequently transmitted to the brain. That impulse created by the odor travels along the olfactory nerve culminating at the glomerulus found within the olfactory bulb of the brain. Multiple impulses are received by each glomerulus that prompt the olfactory receptor interpretation of similar odor particles in the form of a signal. That signal is then transmitted by mitral cells to the olfactory cortex for final signal interpretation. This process is illustrated in Figure 9.[33] 


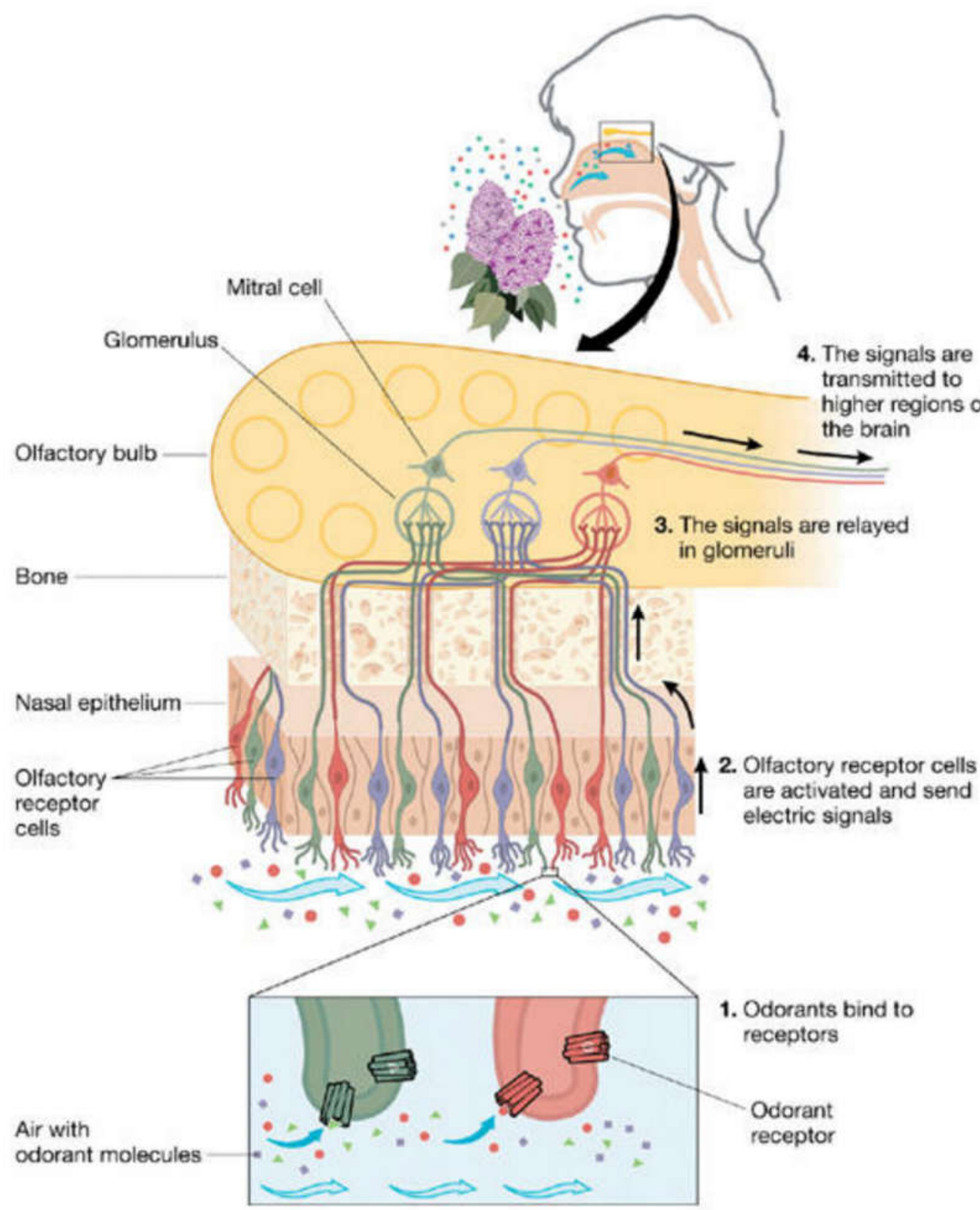

Figure 9 - Illustration of human olfactory system that describes the steps between breathing in odors to brain signal [33]. 


\subsubsection{Canines \& The Justice System}

In the recent past, the science surrounding canine detection of illicit substances has been scrutinized and criticized and has even been referred to as "junk science" [34]-[36]. What critics fail to realize is that canine detection is used as a tool and is not the ultimate cause for conviction. Canines have a far superior sense of smell than that of humans or many other electronic detection system, which is attributed to the large surface area of the olfactory epithelial cells located in the olfactory receptors of the canine's nose [37]. This heightened sensitivity allows canines to locate particular odorants, even in the presence of interfering chemical odorants, such as drugs and explosives [37]. In addition to criminal investigations, canines have been used in other military applications, as well as search and rescue missions because of their strong sense of scent detection.[38]

Canines have been called into routine traffic stops, and there-in lies the potential problem; could this be considered an infringement upon an individual's Fourth Amendment rights? The Fourth Amendment is the right of the citizens of the United States to "be secure in their persons, houses, papers, and effects, against unreasonable searches and seizures[39]".

On June 24, 2006, Officer Wheetley of the Florida Sheriff's office in Liberty County was patrolling with a German shepherd by the name of Aldo, and pulled over an individual by the name of Clayton Harris because of expired license plates[40]. Upon the Officer's approach of the vehicle, he noticed that Clayton Harris was "visibly nervous" as well as an open can of beer in the vehicle's cup holder. Officer Wheetley then asked to search the vehicle to which Mr. Harris refused. The Officer then retrieved Aldo from the patrol car, and proceeded to walk Aldo around the suspect's vehicle. Aldo was trained to 
detect narcotics such as methamphetamine, cocaine, heroin and ecstasy [40]. By the time the canine team reached the driver's side handle, Aldo alerted to the presence of a substance he was trained to detect. Based on that alert, Officer Wheetley concluded that he had sufficient probable cause to search the vehicle and then proceeded to do so. While no illicit substances were found, ingredients for making methamphetamine were present including 200 pills of pseudoephedrine, a bottle of hydrochloric acid, a few containers of anti-freeze and 8,000 matches [41]. After giving Harris his Miranda warnings, he confessed to regularly making methamphetamine at home and even confessed to not being able to go more than a few days without using this narcotic. Based on these confessions, the State charged Harris for possession of pseudoephedrine for the use of manufacturing methamphetamine.[40]

Unfortunately for Harris while out on bail, he was pulled over again by Officer Wheetley for a broken brake light. Officer Wheetley once again deployed Aldo who once again alerted at the driver's side handle. Similarly to the last time, Officer Wheetley presumed to have sufficient probable cause to search the vehicle and upon doing so he discovered nothing of interest.[40]

Harris and his attorney argued that the evidence from his first stop should be thrown out on the ground that an alert from a canine is not sufficient probable cause to search the vehicle. Despite having the qualifications and the required training for both Officer Wheetley and Aldo, they argued that Officer Wheetley did not have complete records of Aldo's performance in routine traffic stops or other field work, proving that the only records the Officer maintained were for those resulting in arrests. Officer Wheetley defended his position by suggesting that Aldo was alerting to "residual odor" left by the 
defendant by transferring the odor of methamphetamine by touching the handle previously.[40]

Faced with these arguments, the trial court concluded that Officer Wheetley did indeed have probably cause to search the defendant's vehicle, and therefore denied the motion to suppress the evidence. Unfortunately, the Florida Supreme Court reversed the decision and claimed that Officer Wheetley lacked probable cause despite Aldo's alert. The Florida Supreme Court argued that despite the fact Aldo was trained and certified, it is not enough to establish probable cause, and instead argued that if the intention was to demonstrate the dog's reliability, it was incumbent on the State to produce a wider array of evidence: "The State must present... the dog's training and certification records, an explanation of the meaning of the particular training and certification, field performance records (including any unverified alerts), and evidence concerning the experience and training of the officer handling the dog, as well as any other objective evidence known to the officer about the dog's reliability. '[40]

In Harris v. Florida, the question was at what point would an alert of a drugdetecting canine to the exterior of an individual's vehicle provide the officer with sufficient probable cause to search the interior of the vehicle without a warrant [41]? Illinois $v$. Caballes already answers the question on whether a canine's alert at the exterior of a vehicle constitutes probable cause for a warrantless search, what Harris v. Florida is arguing against is the reliability of both the canine and the handler [42]. This case found its way to the Supreme Court of the United States and in Florida v. Harris, the Supreme Court Justices unanimously upheld the ruling and argued in favor of the reliability in canine detection [43]. 
There is a misconception with regards to canine detection, in that canines are detecting and alerting to the actual illicit substance, rather they are alerting to a volatile organic compound (VOC), or odorant, that is released from the illicit substance. With cocaine, for example, canines do not alert to the actual cocaine molecule, but rather methyl benzoate, a volatile cocaine byproduct (Figure 10).[44]

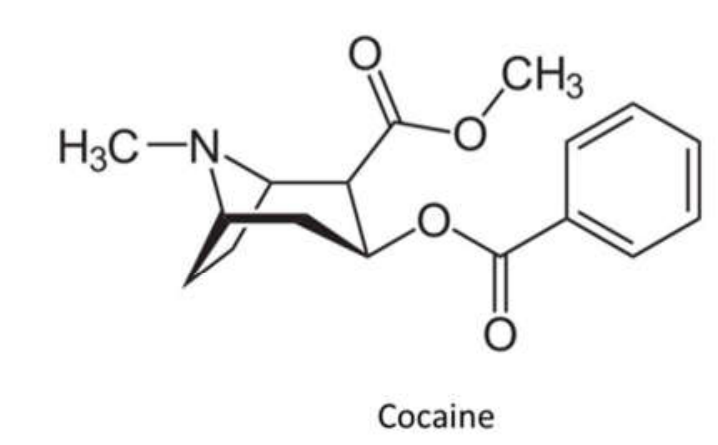

Molecular Formula

Molar Mass

$$
\mathrm{C}_{17} \mathrm{H}_{21} \mathrm{NO}_{4}
$$

$303.353 \mathrm{~g} / \mathrm{mol}$<smiles>COC(=O)c1ccccc1</smiles>

Methyl Benzoate

$\mathrm{C}_{8} \mathrm{H}_{8} \mathrm{O}_{2}$

$136.15 \mathrm{~g} / \mathrm{mol}$

Figure 10 - Difference in chemical structure between cocaine and one of its volatile byproducts, methyl benzoate

The active odor signature, which is defined as a sample containing a chemical that results in an alert from a trained and certified canine [38], have been identified and established for several drugs, such as cocaine, methylenedioxy methamphetamine (MDMA) and methamphetamine [45]-[48]. With explosives, five main explosive families have been evaluated: (1) nitroglycerin (NG) based, (2) smokeless powder based, (3) TNT, (4) tagged and (5) plasticized explosives. A table showing the primary odor of a few narcotics and explosives can be seen in Table 6. Explosives within each class share a common active odor signature, making it possible to train a canine to detect various 
explosives from one class using the corresponding active odor[49]. For this reason, the International Forensic Research Institute (IFRI) at Florida International University developed a training aid kit containing explosive mimics for the use of training canines, without the need to purchase or synthesize illicit substances. This will be discussed in more detail in section 2.3.6.

Table 6 - Examples of illicit substances and their primary odors [2], [21], [48]-[50].

\begin{tabular}{|c|c|}
\hline Illicit Substance & Primary Odor \\
\hline Cocaine & Methyl Benzoate \\
\hline Marijuana & $\beta$-Pinene Caryophyllene \\
\hline Methamphetamine & Benzaldehyde \\
\hline MDMA & Piperonal \\
\hline Polymer Bonded Explosives & 2-ethyl-1-hexanol \\
\hline TNT \& Cast Explosives & 2,4-Dinitrotuluene \\
\hline
\end{tabular}

\subsubsection{Cocaine}

In the research conducted by the Furton research group at the International Forensic Research Institute (IFRI), it was demonstrated that narcotic detecting canines alert to the presence of methyl benzoate, which is a cocaine decomposition product, as the primary odorant for cocaine, while the secondary odor was identified to be benzoic acid (Figure 11) [44], [51]. 


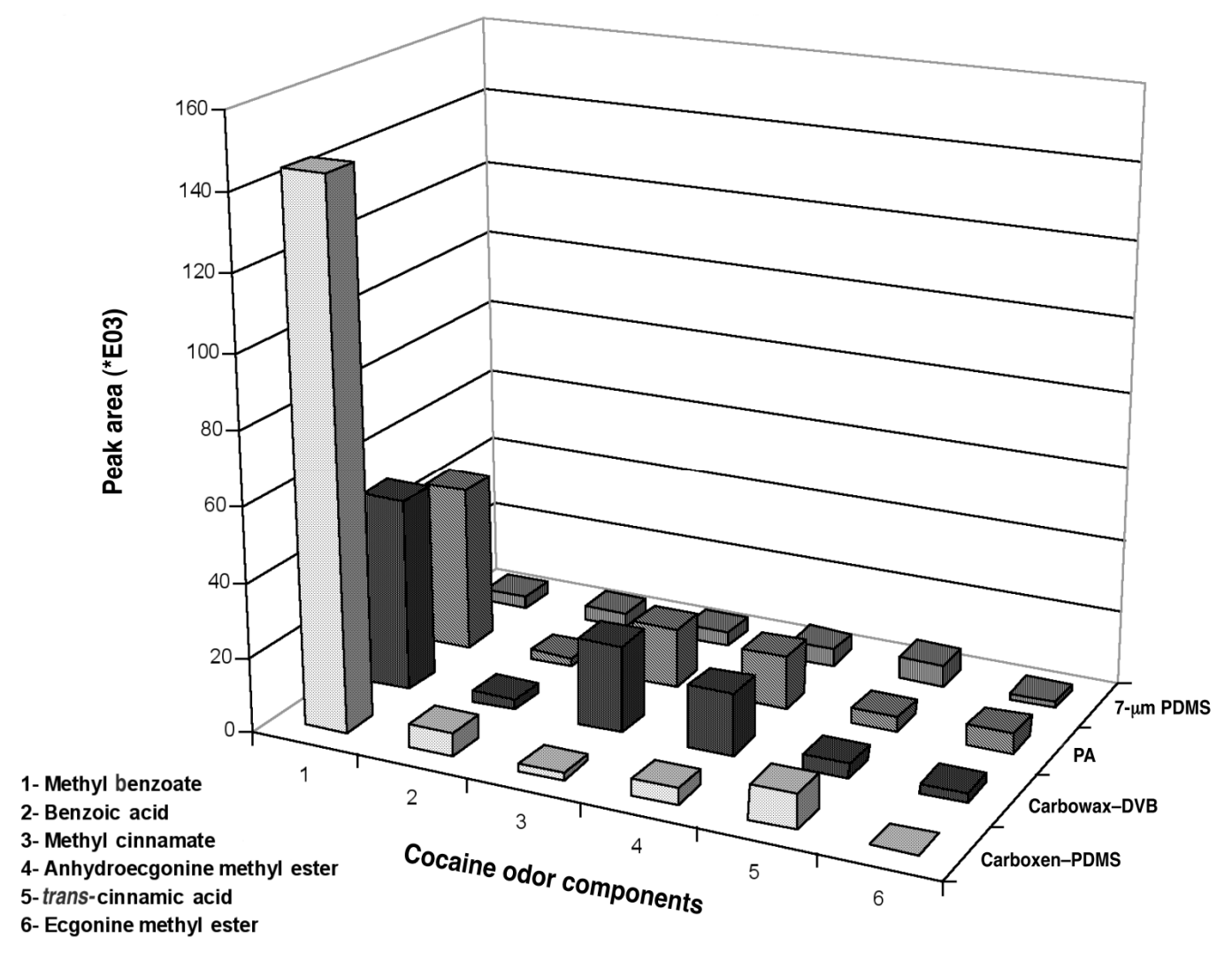

Figure 11 - Comparison of cocaine headspace response by different Solid Phase Microextraction (SPME) fibers, at an exposure time of 12 hours [44].

\subsubsection{Methamphetamine}

In a study conducted by $\mathrm{Vu}$, the headspace of 11 methamphetamine samples were analyzed and 87 different compounds were identified from all samples. Of those 11 samples, 9 of them were illicit methamphetamine seized by law enforcement. As for the remaining two samples, one was synthesized by the DEA and the other was a pharmaceutical-grade. In all of the illicit samples, seven different compounds were found consistently: acetic acid, benzaldehyde, acetophenone, 1-phneyl-2-propanone (P2P), 1phenyl-1,2-propanedione (P12P), 3-phenyl-3-buten-2-one, and 1-chloro-1-phenyl-2propanone. When looking at all 11 samples, only two compounds were commonly found: benzaldehyde and P2P.[48] 


\subsubsection{C-4 (RDX)}

Research has shown that canines will commonly alert to 2-ethyl-1-hexanol, which is a plasticizer, as opposed to RDX, the explosive material [21], [51]-[53]. This applies to both tagged and untagged RDX as shown in Figure 12 [21].

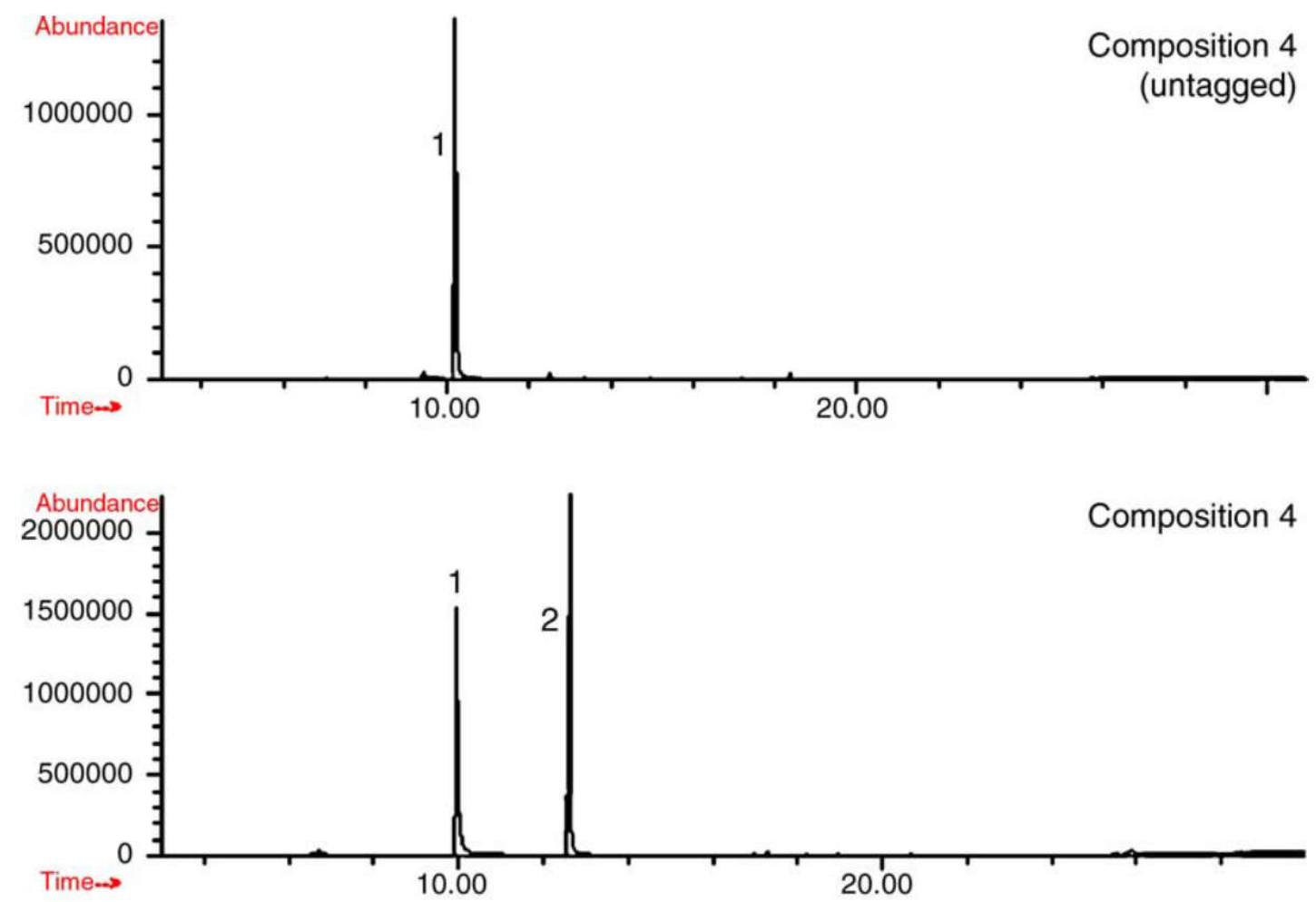

Figure 12 - Headspace analysis of untagged and tagged C-4. Peak 1 is 2-ethyl-1-hexanol and peak 2 is 2,3-dimethyl-dinitrobutane [21].

\subsubsection{Smokeless Powders}

As was discussed in the Explosives section (2.1), smokeless powders can be found as a single base, double base or triple base product. Therefore, finding one smokeless powder that could represent the various options is improbable. In a study conducted by the Almirall group at IFRI, sixty-five smokeless powders were tested using GC and a field deployable ion mobility spectrometer (IMS). It was determined that diphenylamine (DPA), 
ethyl centralite (EC), methyl centralite (MC), 2,4-dinitrotoluene (2,4-DNT), diethyl phthalate (DEP) and dibutyl phthalate (DBP) are the six compounds found in the headspace that would suggest the presence of smokeless powders. The study revealed that DPA was present in the headspace of $96 \%$ of the smokeless powders present.[54]

Table 7 - Compounds found in headspace of smokeless powders and their detection mode [54].

\begin{tabular}{|c|c|}
\hline Compound Name & Detection Mode \\
\hline 2,4-dinitrotoluene & Negative \\
\hline Diphenylamine & Positive \\
\hline Methyl Centralite & Positive \\
\hline Ethyl Centralite & Positive \\
\hline Diethyl Phthalate & Positive \\
\hline Dibutyl Phthalate & Positive \\
\hline
\end{tabular}

\subsubsection{Volatile Organic Compounds and Residual Odors}

As a result of the volatility of these active odorants, VOC's can be transported from one point to another, especially with illicit substances of higher concentrations, and could potentially contaminate porous and non-porous objects that are present within the immediate vicinity. This could become problematic when training or certifying working canines by causing them to give a false positive response, which means that the canine handler identified a response from the canine signifying that there was something present when it fact there was not [38]. In canine trainings and certifications, residual odors could thus increase the potential for false positives or unconfirmed alerts.

Residual odor is defined as the "odor that remains from training aids or actual objects of focus once the aids or objects have been removed" in the Scientific Working Group on Dog and Orthogonal Detector Guidelines (SWGDOG) [38]. Consequently, these 
odors have not only the potential to remain in an area after the illicit subject was removed, but they may also move from one area to another depending on various conditions. Factors such as temperature, humidity and location of a sample during canine training and certifications may contribute to different errors. The concentration or even the amount of drugs or explosives used may also lead to a potential false positive alert on a nearby box. Other factors that could contribute to false errors include the presence of wind, air conditioning, and even the type of materials used (porous vs. non-porous).

Table 8 - Examples of odorants, their molecular formula, weight and structures.

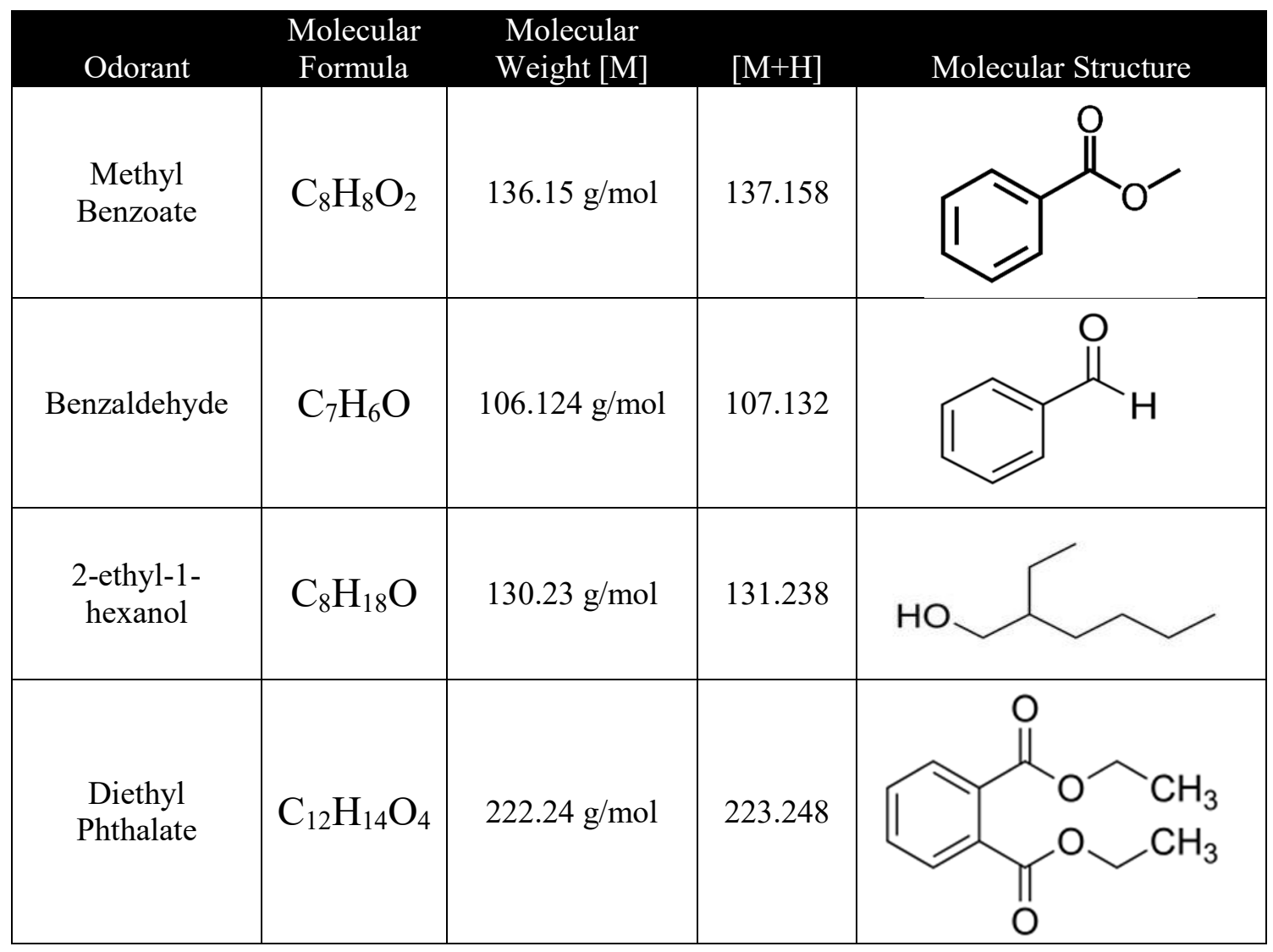




\subsubsection{Training Aids and Controlled Odor Mimic Permeation Systems (COMPS)}

Training aid materials used in canine detection trainings as positive controls are known as surrogate continuation aids (SCAs). The ideal SCA would be safe to handle, harmless to the canine, and able to generate detectable levels of the odorant effectively. Surrogate continuation aids can either be the illicit substance itself, a highly diluted illicit material, or it could be mimics.[49]

Since it has been determined that canines are likely alerting to the presence of volatile organic compounds found in the headspace of illicit substances, training aids that mimic those illicit substances were developed. The idea is to create an imitation product that is similar in functionality to the actual compound. Doing so creates two valuable advantages: (1) it does not contain any controlled substances or explosives, which protect the product from most Alcohol, Tobacco, Firearm and Explosives (ATF) and DEA regulations and control, and (2) it does not expose the canine to harmful narcotics and explosives as they are non-hazardous.[55]

There are various ways to produce mimics or purchase them commercially (e.g., ScentLogix ${ }^{\mathrm{TM}}$ ), however, Controlled Odor Mimic Permeation Systems (COMPS) allow for a controllable, consistent and reliable permeation of the odor [49]. The patented system contains the chemical in either low density polyethylene, polypropylene, cellophane or Kapak plastic bags to allow for permeation of the odor. In an effort to contain the odor further, a "double-bag" design was used along with a non-permeable outer bag that serves as a secondary container (Figure 13).[49] 


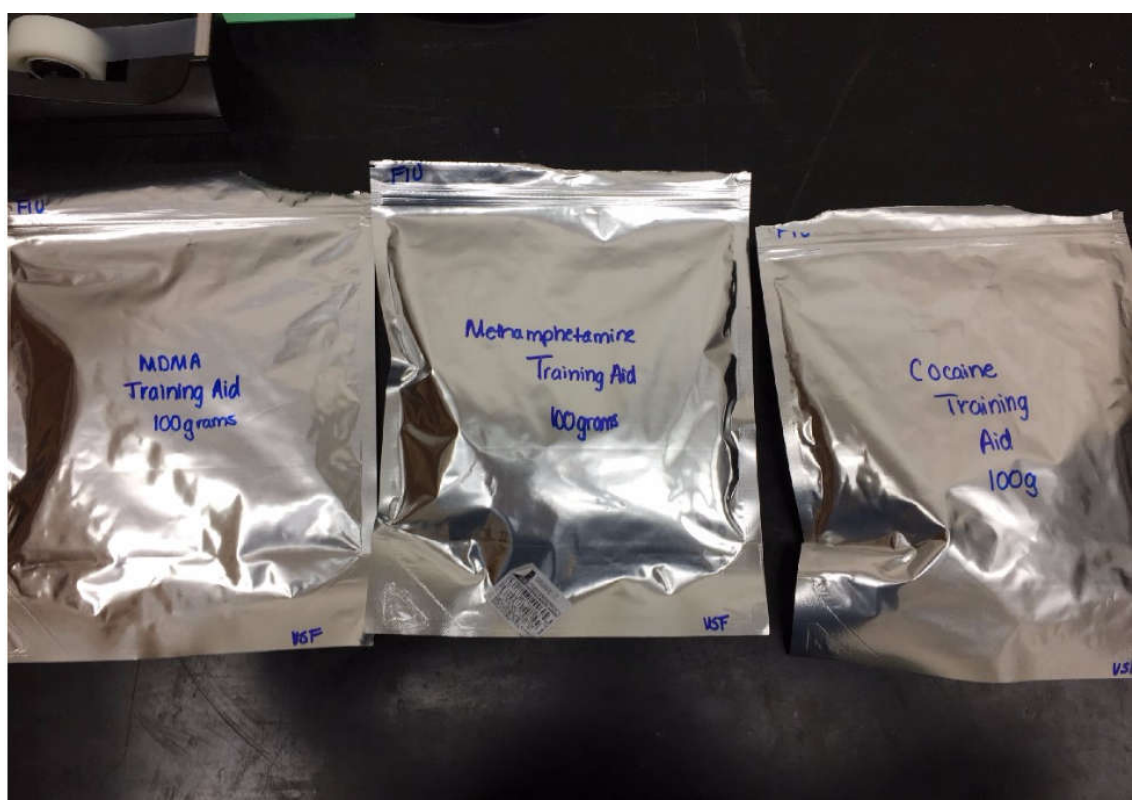

Figure 13 - Picture of the narcotic COMPS made at FIU.

\subsubsection{Aerodynamics of Odor}

In most of the research done involving canine olfaction, there are two flawed assumptions regarding aerial odor plumes. The first involves the assumption that the odor plume is always symmetrically cone shaped as it expands downwind, in addition to assuming that the edges of the plume are clear and distinct [56]. The second assumption is that odors tend to pool in small depressions in the ground at low wind velocities, and that diffusion plays an important role in these circumstances [57], [58]. The reality is these theories are not only incorrect, but incomplete and more research in this area needs to be done [59].

Spatial and temporal scales need to be considered when applying principles of physics to the transport of odors. These factors must also be considered in terms of orderof-magnitude, which would make the spatial scales of centimeters to tens of centimeters 
have comparable fluid dynamics [59]. Similarly, sampling odors from fractions of a second to seconds will yield analogous information.[29]

Spatial scales are split into three intertwined issues. The first refers to the area which the canine's nose samples, which sets the physical parameters of a single information sample [60]. The second scale is the pattern of the canine's movement, which can be separated into two distinct sub-scales: (1) vertical and (2) horizontal movements [61]. In the vertical movement scale, the canine sniffs the ground through various substrates (tiles, carpet, grass, etc.) or as it raises its head slightly to sniff the air, what is occurring is the movement of the canine's nose through aerodynamically different areas of an odor plume. The value of information obtained from sniffing odors at the ground level will vary greatly from odors sniffed a meter above the ground. The horizontal movement scale refers to the pattern in which the canine moves through space, which can range from a simple confined drug search to a larger-scale missing persons search. The third and final scale involves the general area where the odor is dispersed, as the dispersion scale of the odor plume in a room indoors versus a large outdoor area would vary significantly [29], [56], [59].

Unlike the spatial scale, the temporal scale is relatively less complex. Like the spatial scale, there are three scales to consider: (1) the sniffing frequency of the canine, (2) the integration time of the olfactory system and (3) the canine's olfactory memory [62]. These factors are on the order of seconds (sniffing) to minutes (comparison between two or more odor samples).[29], [62], [63] 
The physical processes by which a chemical odor transports from the source to the canine's nose are known as either advection or dispersion. Advection is defined as the "macroscopic, bulk chemical transport by the airflow." Dispersion is the mixture of smaller particles of a substance that are scattered throughout another substance (air in this case). Dispersion is a result of stirring, mixing, and shear dispersion. Within odor plumes, stirring is the process that operates at large spatial scales by redistributing chemicals. While mixing operates at smaller spatial scales by a process that smears chemical gradients. These gradients can be expressed mathematically in Equation 2 where it shows that the movement of chemicals in the direction from greater concentration to less concentration $(q)$ is dependent upon both molecular diffusivity $\left(D_{m}\right)$ and concentration gradients $(\nabla C)$.[29]

$$
q=\quad D_{m} \nabla C
$$

Equation 2 - Flux equation that measures movement of chemicals in direction from greater to less concentration.

Chemicals that are encountered during canine searches have an estimated molecular diffusivity on the order of $10^{-5} \mathrm{~m}^{2} / \mathrm{s}$ (in air). Considering diffusion coefficients can change depending on which chemicals are used, this diffusivity value $\left(10^{-5} \mathrm{~m}^{2} / \mathrm{s}\right)$ is sufficient for the purposes of modeling. What this illustrates is that molecular diffusion is an extremely slow process, and that it should only be considered if there are significant changes in chemical concentrations within very small distances $(\operatorname{large}(\nabla C)$. The average time required for a small particle or molecule to diffuse a distance $(L)$ is shown in Equation 3.[29]

$$
t_{d}=\frac{L^{2}}{2 D_{m}}
$$

Equation 3 - Diffusion time $t_{d}$ required for a small molecule to diffuse over distance $L$. 
When calculating $t_{d}$ for benzaldehyde $\left(D_{m}=0.073 \mathrm{~cm}^{2} / \mathrm{s}\right)$, for instance, at a distance of $0.5 \mathrm{~m}$, it results in a theoretical value of slightly less than 5 hours. Thus, the approach of calculating diffusion for canine searching conditions are ineffective. Furthermore, the use of the Péclet number supports that assertion. The Péclet number is a dimensionless ratio that quantifies the time required for advection to that of diffusion. When Pé is significantly less than 1 , chemical dispersion is heavily affected by molecular diffusion, as is the case in instances of no wind or very short distances. Whereas when Pé is significantly greater than 1 advection dominates as it would for larger distances and windy days. By setting Pé to be equal to 1 , the values at which wind or spatial scales diffusion can be ignored could be solved. Figure 14 shows the values where odor signals are dominated by diffusion versus advection (air flow), and most canine searches occur above the line which indicates that odor signals are a result of advection and not diffusion.[29]

$$
P e ́=\frac{t_{a}}{t_{d}}=\frac{U \quad L}{D_{m}}
$$

Equation 4 - Péclet number where $t_{a}=L / U$ ( $U$ is mean flow velocity of a particular habitat).

Since the movement of air exists at all spatial scales, large and strong air currents (eddies) are observed as wind and thus would transport air toward a principal direction. When the eddies are smaller, they tend to stir the air in an unpredictable manner, which results in odors spreading in an irregular, packet-like way. Therefore when odors traverse in air there will be odor-laden areas of high concentration right next to an area of very low concentration. Meandering, which is the movement of chemicals in different directions from changing wind conditions, is unique to odors and odor plumes as this effect does not exist in light or sound waves.[64] 


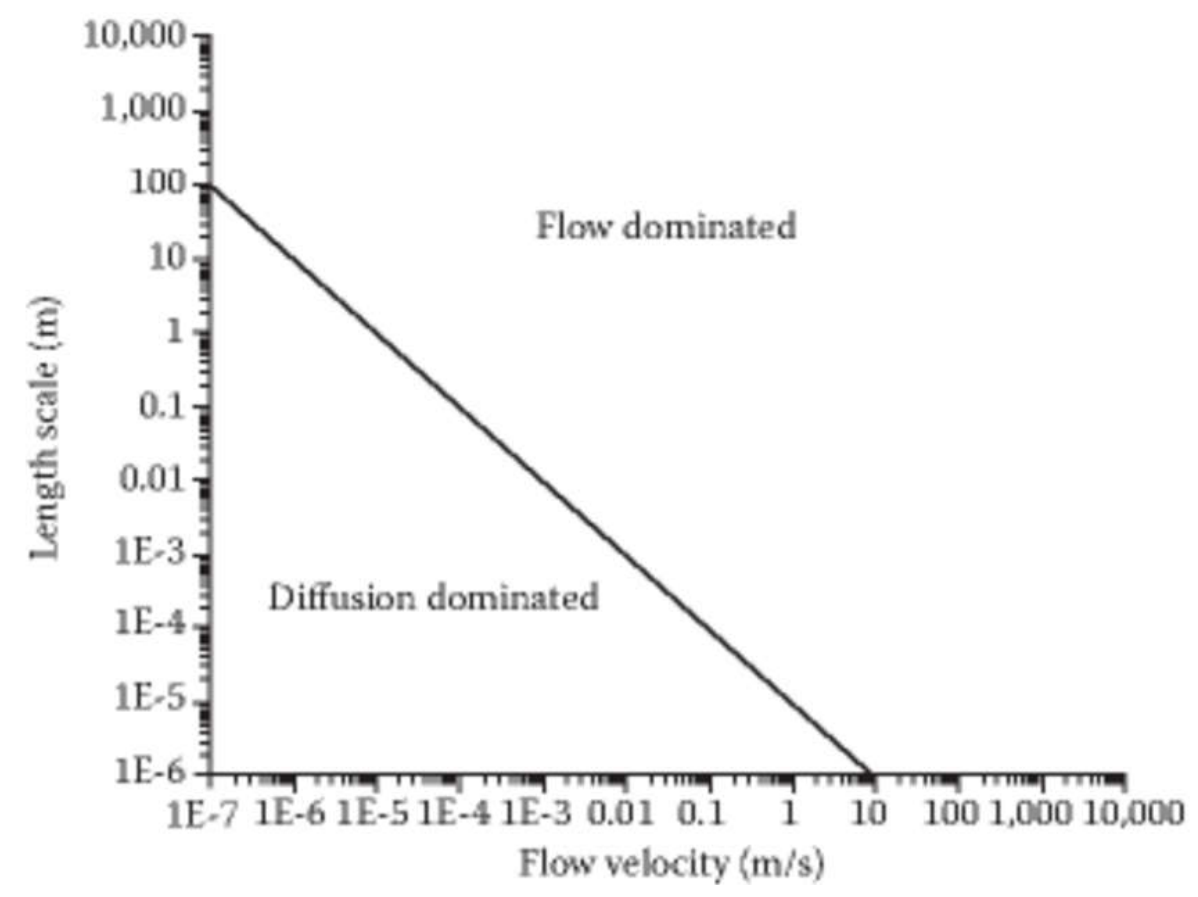

Figure 14 - Graph of Péclet number that illustrates when diffusion dominates advection in its dispersion and vice versa [29].

Since diffusion can therefore be effectively ignored in this context, it becomes apparent that stirring could be the major process involved in the dispersion of chemical signals during canine searches. Stirring processes in a medium (air in this case), are ruled by both the degree and nature of the turbulence in that medium. Estimating the type of turbulence present in a flow is done using the Reynolds number in terms of order-ofmagnitude. The Reynolds number is a ratio of inertial forces with viscous forces and gives a bulk measure of the turbulence (Equation 5). Inertial forces are dominant at the macroscopic scales while viscous forces are dominant in the microscopic levels.[29] 


$$
R e=\frac{U^{2} / L}{v U / L^{2}}=\frac{U \quad L}{v}
$$

Equation 5 - Reynolds number, calculated in terms of order-of-magnitude, ratio of inertial forces $\left(U^{2} / L\right)$ to viscous forces $\left(v U / L^{2}\right)$, where $v$ is the kinematic viscosity of the fluid medium.

Reynolds number, similarly to Péclet, can be discussed in terms of ratios that are significantly less or significantly greater than 1 as they are both ratios of forces. When $\mathrm{Re}$ $>1$, inertial forces are more dominant than viscous forces and therefore the flow is turbulent and eddies are present. Inversely, as viscous forces increase the Reynolds number decreases. Both Péclet and Reynolds numbers are analogous in their interpretation given their similarity as ratios. The kinetic viscosity $(v)$ of air $\left(\right.$ at $\left.20^{\circ} \mathrm{C}\right)=15 \times 10^{-6} \mathrm{~m}^{2} / \mathrm{s}$, is analogous to molecular diffusivity as they are both the denominator of $\mathrm{Re}$ and Pé respectively. The Péclet number compares the rate of advective transport of the chemical to the rate of smearing of gradients and spatial structures in the chemical field. The Reynolds number compares the rate of advective transport due to momentum to the rate of molecular smearing of velocity gradients.[29]

The Schmidt number $(\mathrm{Sc})$ relates the Péclet number to the Reynolds number, which is the ratio of the viscous diffusivity $(v)$ to the mass diffusivity $\left(D_{m}\right)$ (Equation 6$)$. In the spatial scales applicable to canine searches, chemical signals will have a Schmidt number in air between 100-1000 which, relative to the diffusion of momentum, is considered to be weak.[29]

$$
S c=\frac{P e ́}{R e}=\frac{v}{D_{m}}
$$

Equation 6 - Schmidt number is the ratio of the Péclet number to the Reynolds number. 
When examining the interaction between advection and diffusion, it is apparent that diffusion acts to eliminate differences in the concentrations of chemicals along spatial gradients at small spatial scales. That process is referred to as mixing. Since diffusion attempts to homogenize the chemical signal, it also sets a size limit for very small spatial gradients in different concentrations. When sampling is done by a canine in smaller spatial scales than these size limits, it results in an indistinguishable odor signature. Whereas if the sampling occurs at a large spatial scale, the canine can detect differences in concentration. It is then imperative to determine the smallest possible spatial scale of chemical concentration differences. This can be calculated by deriving the smallest velocity structures within a flow using the Kolmogorov scale ( ).[29]

$$
=\left(\frac{v^{3}}{}\right)^{1 / 4}
$$

\section{Equation 7 - Kolmogorov scale equation.}

In the Kolmogorov microscale equation, $v$ is the kinematic viscosity of the fluid and is the rate at which turbulent kinetic energy dissipates into heat. As the dissipation increases, the smallest velocity structures in the flow ( ) becomes smaller. At this point, eddies that are within the flow shrink in size before finally dissipating into heat. Equation 8 describes the Batchelor microscale equation which is the scale of the smallest chemical heterogeneity within a flow. This scale $\left({ }_{B}\right)$ brings chemical diffusion back into the calculation and is used to measure or calculate the smallest detectable differences within a flow.[29] 


$$
{ }_{B}=\left(\frac{v D_{m}^{2}}{1 / 4}\right)^{1 / 4}
$$

Equation 8 - Batchelor mircoscale equation.

By combining the Schmidt (Sc), Kolmogorov ( ) and Batchelor $\left({ }_{B}\right)$ equations, Equation 9 is derived. Again, these numbers and parameters are in terms of order-ofmagnitude and cannot be used for precise numerical calculations. When the Schmidt number is larger than 1 , then the scales of the smallest differences in chemical concentrations are much smaller than the smallest eddies.

$$
{ }_{B}=S c^{-1 / 2}
$$

Equation 9 - Derived equation by combining Schmidt, Kolmogorov and Batchelor equations.

The last aspect regarding the constraints of the physical nature of chemical dispersion is referred to as the boundary layer, which is the interface between the solid structure and the airflow. The dispersion of odors involves the interaction between the airflow and a solid surface, also defined as the "no-slip" condition but is commonly, and technically incorrectly, referred to as "friction" in the canine handling community. Although it is technically incorrect, the concept of "friction" does serve as a useful analogy. Airflow is non-existent at the surface of the structure, but as the distance of the airflow increases away from the solid structure, so does the air velocity (Figure 15). Therefore, the boundary layer is a velocity gradient, and because of the chemical signals being dispersed (primarily) by air movement, the boundary layer has a major impact on the spatial and temporal nature of chemical signals. These chemicals exhibit a streaky persistent characteristic at the near-surface region (wall or floor, etc.) [65], [66]. In turn, as the 
chemicals get further from that surface region, the turbulent energy increases and the eddies become larger in size. With the size increase of eddies, stirring becomes more intense and as a result the chemicals rapidly disperse.[29]

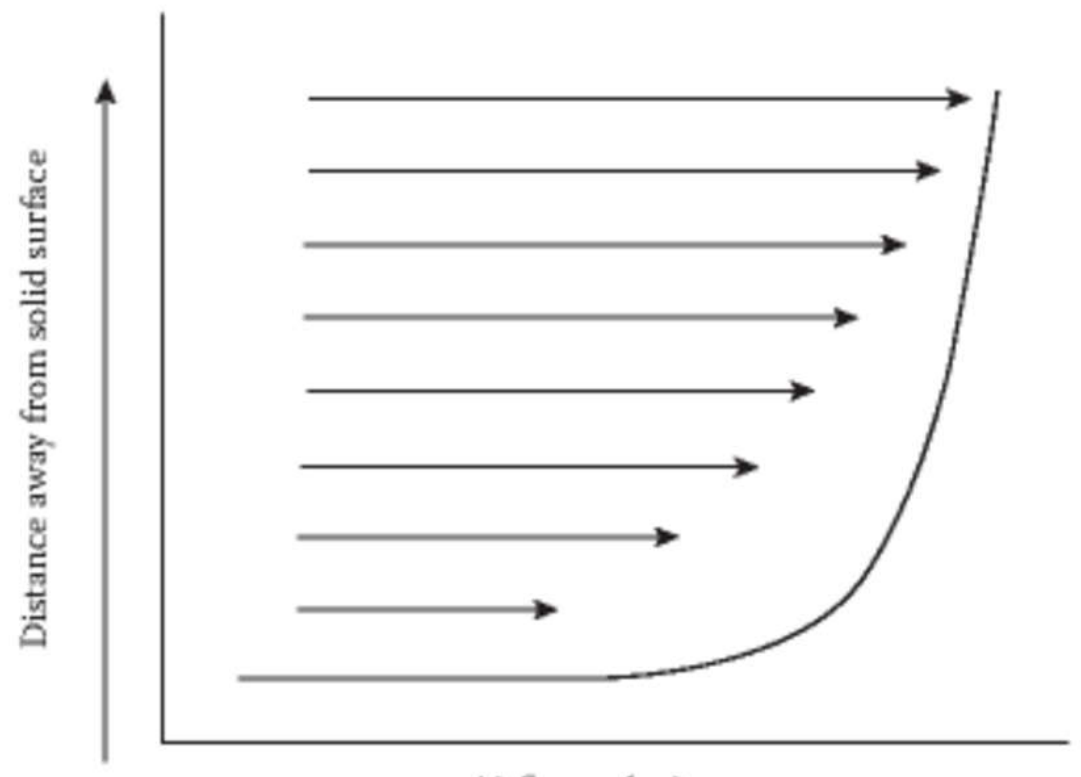

Airflow velocity

Figure 15 - Graph illustrating airflow velocities within a boundary layer [29].

A few important distinctions can be identified after studying the mathematics and physics of odor signals in the air. Firstly, odors at the spatial and temporal scales that are involved in canine searches are distributed by advection, even in open-field outdoor searches, not just in confined searches in a room indoors. Secondly, odor signals and the information they contain are structured by two processes: (1) stirring, which in effect relocates odor patches within a larger plume, and (2) shear dispersion, which primarily functions to 'tear' the odor patches apart into smaller odor patches. While mixing is primarily important for small-scale distances between patches, it mainly serves to 'blur' the edges of odor and non-odor patches. Lastly, the odor information distributed within an 
odor plume can be observed as three distinct phases (Figure 16). In Figure 16, the eddies are larger than the plume at the left-side of the diagram (Phase 1). But as the plume distributes and expands in size to the point where it is the same size as the eddies, the odor filaments within the plume begin to be stirred (Phase 2). As the odor plume expands in size even further, the eddies become smaller and the airflow serves to stir the odor filaments and homogenize the odor within the plume (Phase 3).[29]

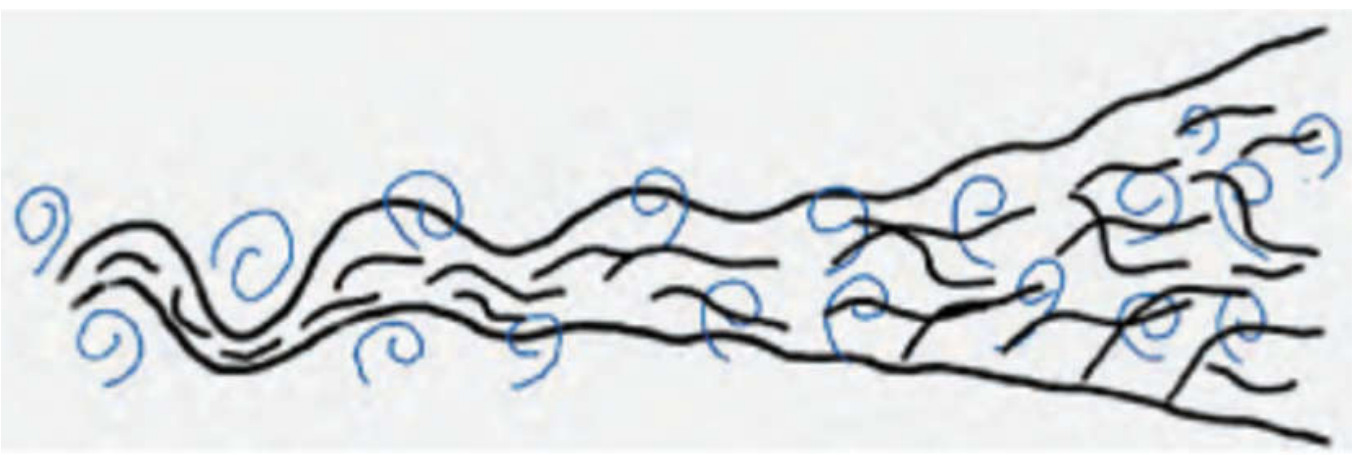

Figure 16 - Picture representing the three distinct phases observed in the distribution of an odor plume. The black lines represent the boundaries of the plume as well as the individual odor filaments within the plume. The blue swirls represent airflow eddies [29].

Phase 1 occurs when the odor is first liberated from its source. The odors spatial distribution is localized around that source; thus, it contains a wide range of eddies that are larger in size than the odor plume. Eddies in turn serves to move the odor plume around but also causes the meandering of the odor plume in space. At this stage, the canine will experience major shifts in terms of concentration as they will detect high concentration levels to no plume at all, and they will revert to large lateral movements to try and locate the odor plume.[29]

Phase 2 is the phase in which the odor plume begins to expand in size equal to that of the turbulent eddies within the airflow. Shear dispersion is the dominant dispersal 
process in this phase, and this causes the odor patches to be torn apart into smaller patches and distributed in space. Instead of moving the plume as a whole, this phase creates odor filaments from the odor plume, still keeping a high concentration boundary between parcels of air with and without odors. This creates a rapid fluctuation in concentration of the odor plume which would make the canine stop and take several sniffs at a particular point in space.[29]

Phase 3 is where the odor plume is much larger than the largest eddies, and mixing is the dominant dispersal process. At this point of the phases, the plume becomes homogenous as the concentration differences between air packets that are odor-laden and those that are odorless become blurred. During this phase of plume development, the canine will experience a slowly fluctuating yet constant signal, and the concentrations of the odor rarely varies while the differences in signal concentration across space are practically nonexistent.[29]

\subsection{Instrumentation}

Mass spectrometry has been a rapidly-growing area of analytical chemistry because of its high sensitivity and selectivity, especially when compared to other spectrometric methods [67]. It is used in various fields for various purposes. It is also very universal in that different interfaces can be used to introduce the sample. Some mass spectrometers can be coupled to varying chromatographic techniques such as gas chromatography (GC), liquid chromatography (LC), and ion chromatography (IC) [67], [68]. Unfortunately, the various ion sources that are currently available place several restrictions on the convenience and rapidity of sample introduction and its subsequent analysis [69]. 
Ion sources that are used currently in mass spectrometry require a high vacuum system for sample introduction. These ion sources include electron ionization (EI), chemical ionization $(\mathrm{CI})$, fast atom bombardment (FAB), and field desorption/field ionization $(\mathrm{FD} / \mathrm{FI})$. Although these methods have been around for quite some time, they require that the samples be introduced in high vacuum which can be viewed as a severe limitation. Gaseous or liquid samples are introduced through a chromatograph or specially designed inlet system, whilst solid samples are introduced using a direct insertion probe and a vacuum lock system.[69]

Direct Analysis in Real Time (DART) is a soft ionization source, which utilizes a gas stream to ionize water molecules in the air which in turn ionize the sample molecules [69]. The DART is coupled to an accurate mass time-of-flight mass analyzer (Accu$\mathrm{TOF}^{\mathrm{TM}}$ ). Following ionization of the sample ions, the ions then travel to the Accu-TOF ${ }^{\mathrm{TM}}$ into an opening referred to as orifice 1 . By raising the voltage of orifice 1, fragmentation of ions will occur via collision induced dissociation. The ions are then sent to a high frequency ion guide, which is quadrupole [70]. The purpose of the quadrupole is to allow ions to pass through to orifice 3, while other unwanted molecules get drawn to the turbo pumps below.

The DART ion source was invented by Robert "Chip" Cody in 2003. It was operational in January of that year and the patent was filled a few months later in April. The product was available commercially in March of 2005. It was the first open-air, ambient ion source for mass spectrometry [69]. Whilst maintaining many advantages over the various chromatographic techniques when coupled to mass spectrometers, DART still has disadvantages in regards selectivity, detection limits and sensitivity of certain samples. 
It's also useless when analyzing large bio-molecules such as proteins. In addition, it does not ionize metals and minerals [67].

It has been validated for the testing of explosives, accelerants, inks, dyes and more [20], [69], [71]-[85]. The instrument is capable of ionizing liquids, solids and gaseous state samples. For liquids, the sample may be introduced to the gas stream via dipping the closed end of a capillary tube into the solution, and then placing it into the gas stream. Volatile substances can be easily analyzed by simply ionizing the headspace of the sample. Solid samples are ionized when introduced to the gas stream, albeit at a much higher temperature. One of the limitations of the instrument, however, is the minimal sampling area in between the DART source and the orifice 1.

\subsubsection{Direct Analysis in Real Time}

The DART ion source (Figure 17) is composed of a gas inlet, discharge chamber, gas heater and different electrodes (needle, grounded, etc.). The gas can be either nitrogen or helium, although helium is the most common. The discharge chamber contains a cathode and an anode. By applying several kilovolts of electrical potential, an electrical discharge initiates a production of ions, electrons and excited-state species in a plasma. The end of the ion source is a ceramic insulator cap, which directs the helium gas stream into the open air sample gap.[67] 


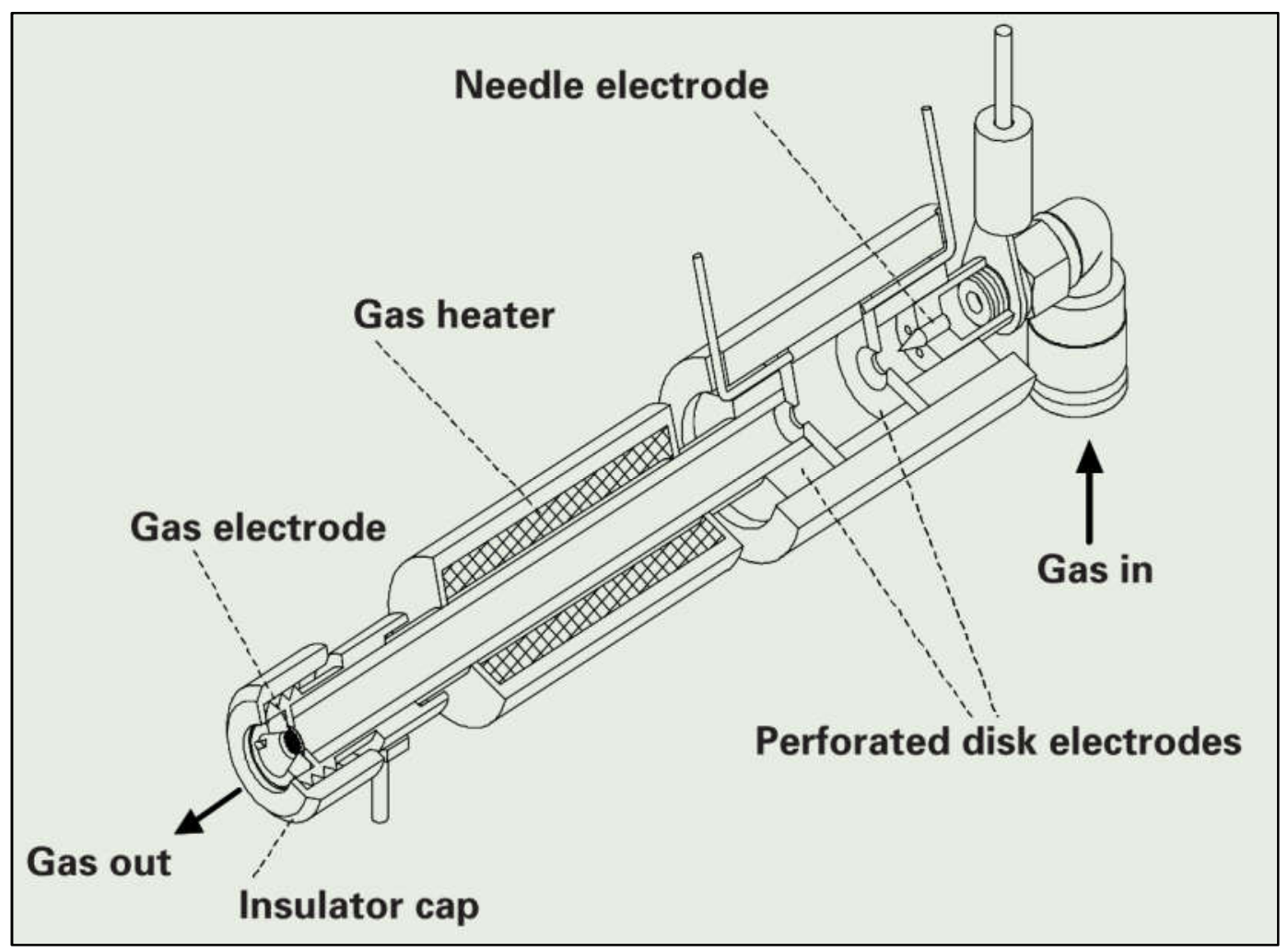

Figure 17 - Schematic diagram of the DART ion source [67].

\subsubsection{Ionization}

When a sample is introduced, the sample is ionized directly by energy transfer from metastables $\left(\mathrm{M}^{*}\right)$. For proton transfer (positive ions), metastable helium ionizes atmospheric water, and in turn the ionized water clusters transfer protons to the sample. This process is referred to as Penning Ionization, and is the simplest form of the several ionization mechanisms. [69]

$$
\mathrm{M}^{*}+\mathrm{S} \rightarrow \mathrm{S}^{+}+\mathrm{M}+\text { electron }
$$

For a reaction to occur, metastable atoms or molecules react only with analytes that possess an ionization potential less than that of the metastable energy. The helium $2^{3} \mathrm{~S}$ state has $19.8 \mathrm{eV}$ of internal energy, whereas most molecules have ionization energies much lower than the helium $2^{3} \mathrm{~S}$ state of $19.8 \mathrm{eV}$. 


$$
\begin{gathered}
\mathrm{He}\left(2^{3} \mathrm{~S}\right)+\mathrm{H}_{2} \mathrm{O} \rightarrow \mathrm{H}_{2} \mathrm{O}^{+\cdot}+\mathrm{He}\left(1^{1} \mathrm{~S}\right)+\text { electron }^{-} \\
\mathrm{H}_{2} \mathrm{O}^{+\cdot}+\mathrm{H}_{2} \mathrm{O} \rightarrow \mathrm{H}_{3} \mathrm{O}^{+}+\mathrm{OH} \\
\mathrm{H}_{3} \mathrm{O}^{+}+\mathrm{n} \mathrm{H}_{2} \mathrm{O} \rightarrow\left[\left(\mathrm{H}_{2} \mathrm{O}\right) n \mathrm{H}\right]^{+} \\
{\left[\left(\mathrm{H}_{2} \mathrm{O}\right) n \mathrm{H}\right]^{+}+\mathrm{M} \rightarrow \mathrm{MH}^{+}+\mathrm{nH}_{2} \mathrm{O}}
\end{gathered}
$$

For electron capture (negative ions), penning electrons are rapidly thermalized, oxygen from the atmosphere then captures the electrons and in turn the $\mathrm{O}_{2}^{-}$ionizes the sample thus producing anions.[69]

$$
\begin{gathered}
\mathrm{M}^{*}+\text { surface } \rightarrow \mathrm{M}+\text { surface }+\mathrm{e}^{-} \\
\mathrm{e}_{\text {fast }}^{-}+\text {gas } \rightarrow \mathrm{e}_{\text {slow }}^{-} \\
\mathrm{e}_{\text {slow }}^{-}+\mathrm{O}_{2} \rightarrow \mathrm{O}_{2}^{-}
\end{gathered}
$$

There are, however, limitations to ionization and atmospheric ion transport associated with physiochemical phenomena. Thus, it makes it difficult to use as a quantitative technique. Harris and Fernandez found that the way a solid sample is held in the stream, in addition to where in that gap it is held, affects the ionization and ultimately the results [86]. In their study, they used computer simulations and particle tracing plots of a circular acetaminophen tablet in different positions and orientations to determine the optimal sample placement (Figure 18). They used three positions relative to the sample gap, (1) adjacent to the ion source $(0 \mathrm{~cm}),(2)$ between the ion source and orifice $1(1.5 \mathrm{~cm})$ and $(3)$ adjacent to the orifice $(3 \mathrm{~cm})$. In addition, three other positions were studied relative to the DART stream: (1) above the stream, (2) middle of the stream and (3) below the DART stream. The three orientations used were (1) sideways, (2) flat and (3) upright. They concluded that placement close to the ion source, in an upright position and slightly below the gas stream is the optimal sampling technique, for tablets at least.[86] 
a)
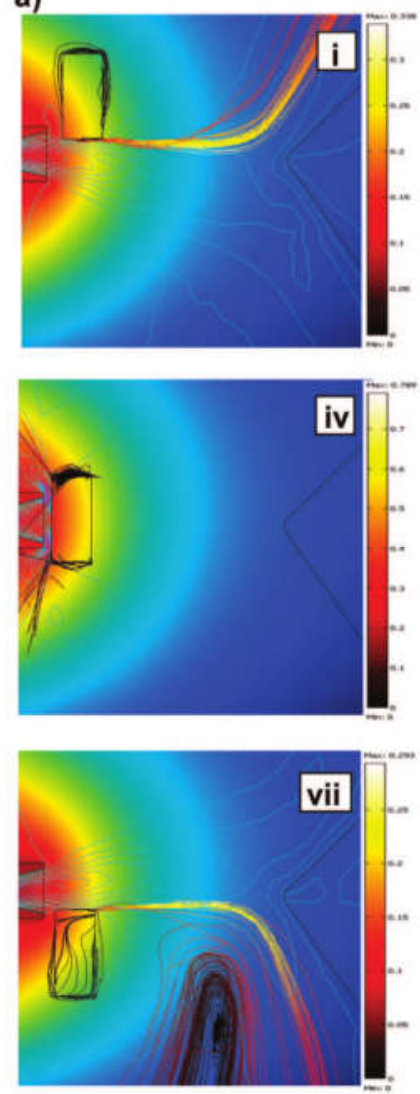
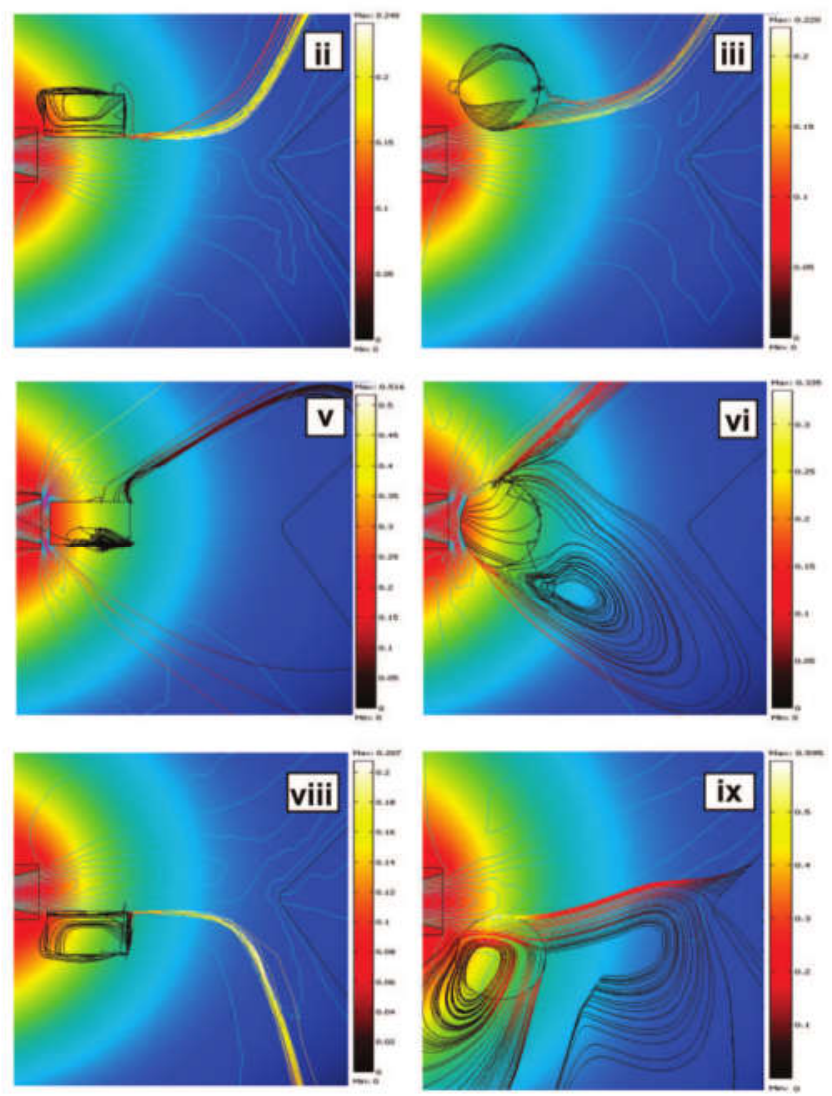

b)

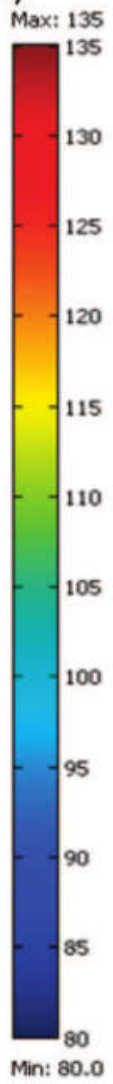

Figure 18 - (a) Particle tracing simulation of circular acetaminophen tablet at simulated particle velocities $(\mathrm{m} / \mathrm{s})$ and $(\mathrm{b})$ temperatures and scale $\left({ }^{\circ} \mathrm{C}\right)$. Top row: Above position: (i) sideways, (ii) flat and (iii) upright. Middle row: Inline position: (iv) sideways, (v) flat and

(vi) upright. Bottom row: Below position (vii) sideways, (viii) flat and (ix) upright [86].

\subsubsection{Collision Induced Dissociation}

Following ionization of the sample, the ions are then drawn to the spectrometer inlet, which is referred to as orifice one. The potential of this orifice can be adjusted by the user. Typically, an orifice voltage of 20 or 30 volts is used. At higher voltage values, fragmentation of ions will occur. Fragmentation occurs as a result of collision induced dissociation which is defined as "an ion/neutral species interaction wherein the projectile ion is dissociated as a result of interaction with a target species. This is brought about by 
conversion of part of the translation energy of the ion to internal energy in the ion during collision.’[87]

\subsubsection{Function Switching}

The software allows for "function switching", which allows for the collection of spectra at varying orifice voltages simultaneously. For example; the user can define a function switching method which would allow for the collection of 3 spectra at varying orifice 1 voltages of 30, 60 and 90 volts. The disadvantage, however, would be a decrease in sensitivity. Function switching is ultimately useful when attempting to differentiate or distinguish two different compounds with similar or even exact masses. For example, cocaine and scopolamine both have $m / z 304.1548$ and molecular formula $\mathrm{C}_{17} \mathrm{H}_{22} \mathrm{NO}_{4}$. By increasing the orifice 1 voltage and thus creating collision induced dissociation, fragment ions will differentiate the two compounds. Scopolamine fragments at m/z 138 and 156 whereas cocaine fragments at m/z 82 and 182 (Figure 19 and Figure 20).

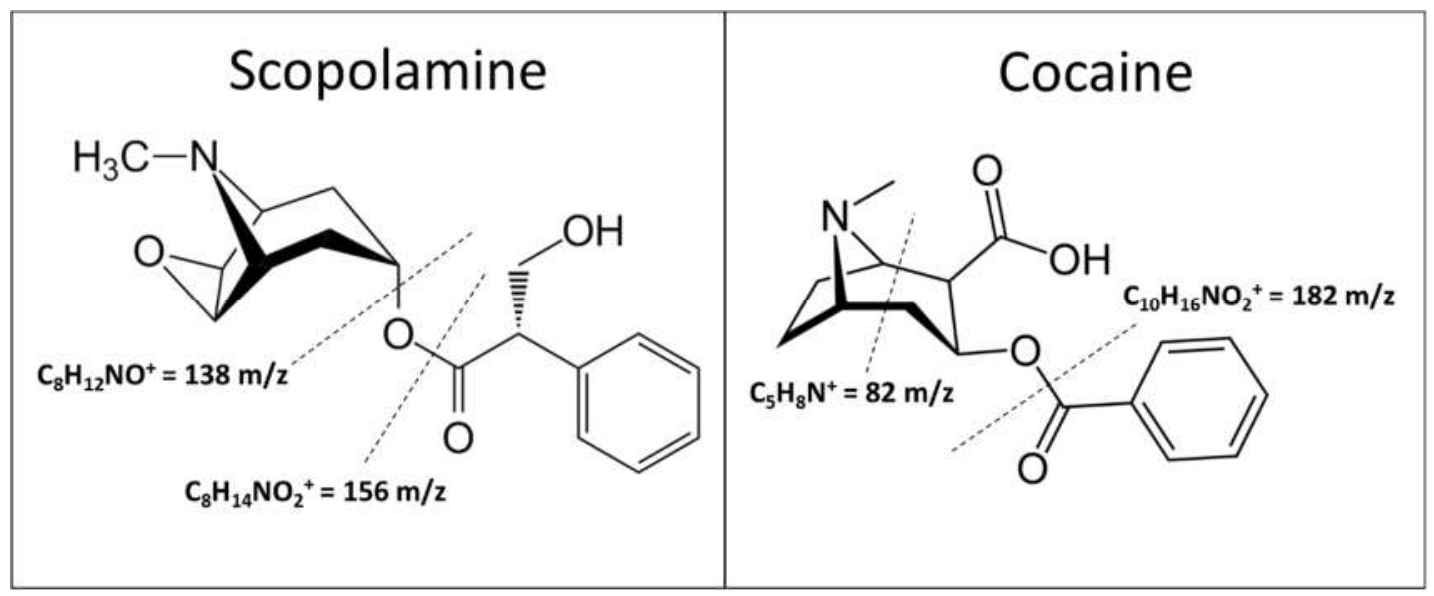

Figure 19 - The different fragment ions created in scopolamine and cocaine. 


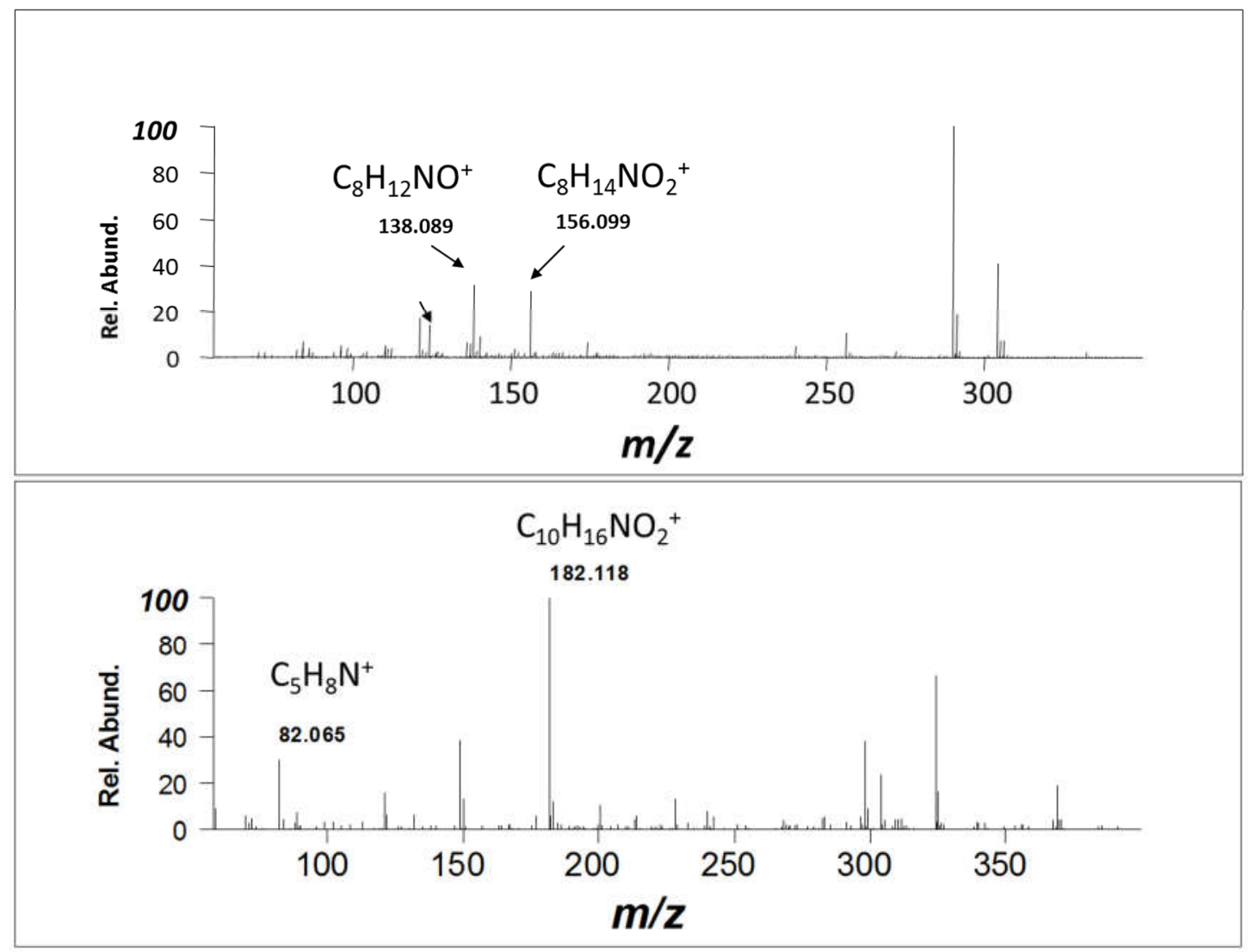

Figure 20 - Mass spectra for scopolamine (top) showing the fragment ions that would distinguish it from an unknown vs. cocaine (bottom) [69].

\subsubsection{Mass Spectrometer}

A variety of different mass spectrometers are available. Scanning types include magnetic sector, quadrupoles and triple quadrupoles. Trapped ion types include Fourier transform, 3D ion trap, Orbitrap and linear trap. Then there are mass spectrometers referred to as "time-of-flight" (TOF) which separate ions based on their size.[70]

Signals created by DART can be transient, therefore scanning mass spectra works best with selected ion monitoring or 'fast scanning.' Selected reaction monitoring on triple quadrupole mass spectrometers is good for target compound quantitation. Ion traps work, but are not a good choice for quantitative analysis. Time-of-flight is fastest mass 
spectrometer for transient signals, and it also provides ultra-high resolution data for the entire mass spectrum with minimal-to-no sensitivity loss. The theoretical mathematical equation describing the movement of ions in the time-of-flight spectrometer is shown in Equation 10. The flight time is inversely proportional to the square root of the mass/charge $(\mathrm{m} / \mathrm{z})$ ratio (Equation 11$)$.

$$
\frac{1}{2}\left(M m_{u}\right) v^{2}=q \quad e \quad V
$$

Equation 10 - Time-of-flight mathematical equation where $M$ is the mass of the ion, $m_{u}$ is atom mass unit $\left(1.6605 \times 10^{-27} \mathrm{~kg} / \mathrm{u}\right), \mathrm{v}$ is flight speed of ion, $q$ is charge number of ion, $e$ is unit electric charge $\left(1.602 \times 10^{-19}\right.$ and $\mathrm{V}$ is accelerating voltage.

$$
\text { tof } \propto \frac{L}{\sqrt{V}} \sqrt{\frac{q}{M}}
$$

Equation 11 - Equation describing the inverse relationship between flight time and square root of $\mathrm{m} / \mathrm{z}$.

The DART-AccuTOF contains a quadrupole, which is used for ion transport in addition to getting rid of unwanted ions, and a time-of-flight mass analyzer (Figure 21).[70] 


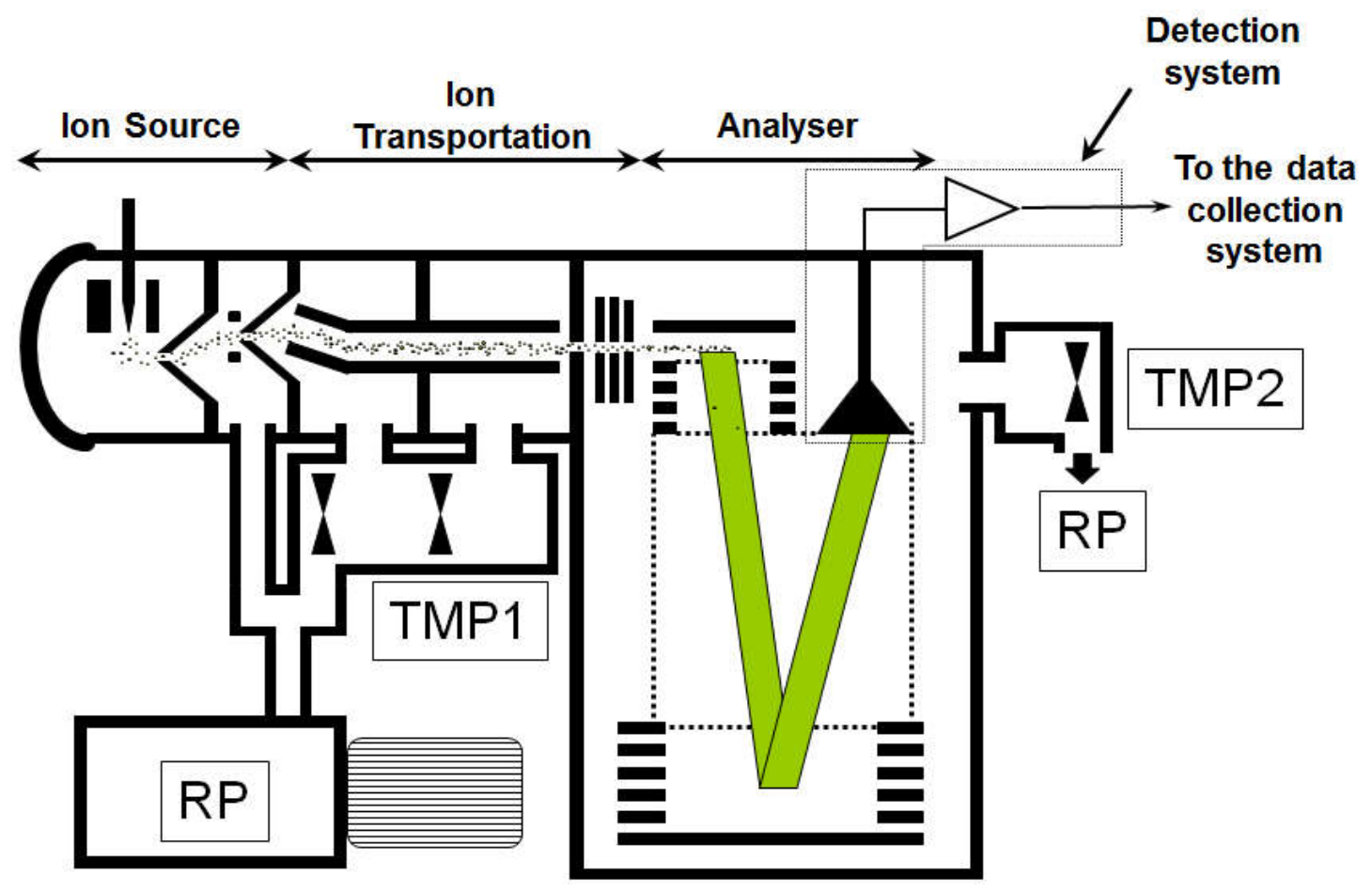

Figure 21 - Schematic of JMS-T100LC AccuTOFTM [70].

\subsubsection{Ion Transportation}

As ions pass through orifice 1 , they are then attracted towards orifice 2 which is intentionally misaligned with orifice 1 (Figure 21). The main purpose is to filter unwanted ions and molecules. Seeing that the ionization is taking place in open-air, many unwanted ions enter through the orifice 1 opening and will thus bounce off of the orifice 2 apparatus, and in turn be drawn towards the rough pump.[70]

Once the ions pass through orifice 2 , they proceed to the ion transport region. The ion transport region is a high-vacuum region which allows for a strong acceleration of ions to occur, minimizing collision induced dissociation and scattering. There are three various types of high frequency ion guides that can be used; quadrupoles, hexapoles, and octupoles. 
Of the three, quadrupoles are preferred due to the power in which it focuses ions toward the central axis. This helps in accomplishing both high resolution and sensitivity. The disadvantage, however, is that quadrupoles have the narrowest mass to charge ratio $(\mathrm{m} / \mathrm{z})$ range for the transmission of ions simultaneously. This is not an issue because of the combination of the ion guide with a conventional quadrupole MS (QMS) or magnetic field MS.[70]

\subsubsection{Mass Analyzer}

The quadrupole, which is bent, acts as an ion guide, focusing the ions of interest through to orifice 3 . The quadrupole is composed of four parallel cylindrical electrode rods. Each pair of opposing rods is connected electrically. One pair is connected to the positive side of a variable dc source while the other is connected to the negative side. Both pairs are also connected to variable radio-frequency ac voltages. When the ions are accelerated into the space in between the pair of rods, the ac/dc voltages on the rods are increased simultaneously, maintaining a constant ratio. Only a certain range of $\mathrm{m} / \mathrm{z}$ ions will go through to the end of the quadrupole while others strike the rods and convert to neutral molecules. The quadrupole in this instrument acts as a "high-pass" filter, allowing ions greater than the chosen $\mathrm{m} / \mathrm{z}$ to go through. The spatial focus that is caused by the quadrupole allows for good resolution.[70], [88]

\subsubsection{Ion Focusing Lenses}

Once the selected $\mathrm{m} / \mathrm{z}$ ions pass through the quadrupole and through orifice 3 , they pass through a series of multi-function focusing and steering lenses. The purpose of these lenses is to ensure that the ions travel towards the time-of-flight mass analyzer in the same level. They essentially push the ions together to allow for a collimated stream of ions to 
enter the mass analyzer, and to be repelled by the pusher plate simultaneously. This allows for reproducible separation in the TOF tube.[70]

\subsubsection{Time-of-Flight Mass Analyzer}

The flight cycle of ions in the time-of-flight tube consists of many steps. First, various ions are introduced at the same time. When the pulser voltage is applied on the pusher plate, all ions begin to travel in the time-of-flight tube. The mass analyzer of the instrument incorporates a single stage reflectron. The ions that were generated from the ion source are introduced to the flight tube and by applying varying voltages, ions then separate based on their $\mathrm{m} / \mathrm{z}$ ratios, reflected in the reflectron and onto a detector. Ions with low $\mathrm{m} / \mathrm{z}$ reach the detector first.[70], [88]

\subsubsection{Detector}

The detector is a dual micro channel plate (MCP) detector. These detectors are extremely delicate, both surfaces of the MCP are metal coated that serves as electrodes.[70] They have a diameter of $40 \mathrm{~mm}$ and a thickness of $0.6 \mathrm{~mm}$. The I.D. of each channel is 10 $\mu \mathrm{m}$ and the gap of each channel is $12 \mu \mathrm{m}$. The purpose of these plates is to amplify the electron signal, in a similar fashion as electron multipliers. The major difference, however, is that micro channel plates have several million independent channels, wherein each channel works as an electron multiplier. When an ion, electron or photon enters the channel, an electron will emit from the channel wall. Following that, secondary electrons accelerate due to a voltage being applied which creates an electric field across the MCP. As this process repeats over and over again, it yields several thousand electrons as a result. 


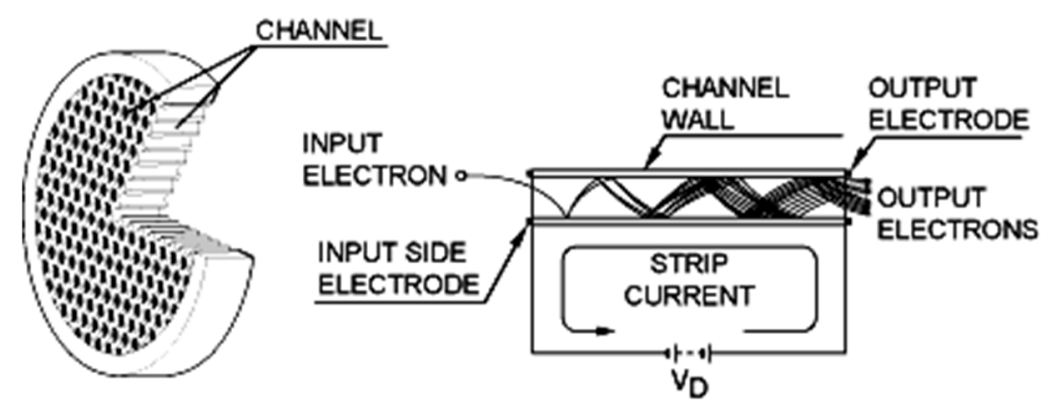

Figure 22 - Schematic of a micro channel detector [89].

Some advantages of these micro channel plates are high gain, and high spatial and temporal resolution. Generally, the detector consists of three major parts: A converter, an assembly of MCP's and a read-out device. Because the instrument requiring a detector with an amplification factor of $10^{6}$, a dual MCP (two MCP's layered) is used.[70], [89]

\subsubsection{Data Acquisition}

The analysis occurs in real time, in that the data acquisition and reporting occurs simultaneously with the sample introduction. This feat is partly a result of changing the data acquisition system from Time-to-Digital Converters (TDC) to Analog-to-Digital Converter (ADC). This also allows for achieving a wider dynamic range, exceeding four orders of magnitude. This is achieved without losing any of its advantages such as high resolution, high mass accuracy, high sensitivity, and its wide mass range. When TDC is used as a data acquisition system, the quality of the mass spectra will rarely be affected by the ion detection system. Whereas when ADC or the continuous digital average is used, the ion detection system directly affects the quality of the mass spectra. However, the ADC/signal average of this AccuTOFTM instrument was designed to minimize signal distortion.[70] 


\subsubsection{Calibration}

One of the major benefits of the instrument is the ability to calibrate each data file. Calibration is done using neat polyethylene glycol average molecular weight 600 (PEG 600 ) in the beginning or end of each run. When the instrument is in positive-ion mode, the PEG 600 produces a series of $\left[\mathrm{M}+\mathrm{H}-\mathrm{H}_{2} \mathrm{O}\right]^{+}$peaks ranging from $m / z 45-1000$. These peaks, identified in (Table 9) are then used as a reference spectrum as can be seen in (Figure 23).

Table 9 - Exact mass of each polyethylene glycol (PEG) peak [90].

\begin{tabular}{|c|c|}
\hline Exact Mass & Composition \\
\hline 65.06025 & {$\left[\left(\mathrm{CH}_{3} \mathrm{OH}\right)_{2}+\mathrm{H}\right]^{+}$} \\
\hline 107.07081 & {$\left[\left(\mathrm{C}_{2} \mathrm{H}_{4} \mathrm{O}\right)_{2}+\mathrm{H}_{2} \mathrm{O}+\mathrm{H}\right]^{+}$} \\
\hline 151.09703 & {$\left[\left(\mathrm{C}_{2} \mathrm{H}_{4} \mathrm{O}\right)_{3}+\mathrm{H}_{2} \mathrm{O}+\mathrm{H}\right]^{+}$} \\
\hline 195.12324 & {$\left[\left(\mathrm{C}_{2} \mathrm{H}_{4} \mathrm{O}_{4}+\mathrm{H}_{2} \mathrm{O}+\mathrm{H}\right]^{+}\right.$} \\
\hline 239.14946 & {$\left[\left(\mathrm{C}_{2} \mathrm{H}_{4} \mathrm{O}\right)_{5}+\mathrm{H}_{2} \mathrm{O}+\mathrm{H}\right]^{+}$} \\
\hline 283.17567 & {$\left[\left(\mathrm{C}_{2} \mathrm{H}_{4} \mathrm{O}\right)_{6}+\mathrm{H}_{2} \mathrm{O}+\mathrm{H}\right]^{+}$} \\
\hline 327.20189 & {$\left[\left(\mathrm{C}_{2} \mathrm{H}_{4} \mathrm{O}\right)_{7}+\mathrm{H}_{2} \mathrm{O}+\mathrm{H}\right]^{+}$} \\
\hline 271.22810 & {$\left[\left(\mathrm{C}_{2} \mathrm{H}_{4} \mathrm{O}\right)_{8}+\mathrm{H}_{2} \mathrm{O}+\mathrm{H}\right]^{+}$} \\
\hline 415.25432 & {$\left[\left(\mathrm{C}_{2} \mathrm{H}_{4} \mathrm{O}\right)_{9}+\mathrm{H}_{2} \mathrm{O}+\mathrm{H}\right]^{+}$} \\
\hline 459.28053 & {$\left[\left(\mathrm{C}_{2} \mathrm{H}_{4} \mathrm{O}\right)_{10}+\mathrm{H}_{2} \mathrm{O}+\mathrm{H}\right]^{+}$} \\
\hline 503.30675 & {$\left[\left(\mathrm{C}_{2} \mathrm{H}_{4} \mathrm{O}\right)_{11}+\mathrm{H}_{2} \mathrm{O}+\mathrm{H}\right]^{+}$} \\
\hline 547.33296 & {$\left[\left(\mathrm{C}_{2} \mathrm{H}_{4} \mathrm{O}\right)_{12}+\mathrm{H}_{2} \mathrm{O}+\mathrm{H}\right]^{+}$} \\
\hline 591.35918 & {$\left[\left(\mathrm{C}_{2} \mathrm{H}_{4} \mathrm{O}\right)_{13}+\mathrm{H}_{2} \mathrm{O}+\mathrm{H}\right]^{+}$} \\
\hline 635.38539 & {$\left[\left(\mathrm{C}_{2} \mathrm{H}_{4} \mathrm{O}\right)_{14}+\mathrm{H}_{2} \mathrm{O}+\mathrm{H}\right]^{+}$} \\
\hline 679.41161 & {$\left[\left(\mathrm{C}_{2} \mathrm{H}_{4} \mathrm{O}\right)_{15}+\mathrm{H}_{2} \mathrm{O}+\mathrm{H}\right]^{+}$} \\
\hline 723.43782 & {$\left[\left(\mathrm{C}_{2} \mathrm{H}_{4} \mathrm{O}\right)_{16}+\mathrm{H}_{2} \mathrm{O}+\mathrm{H}\right]^{+}$} \\
\hline 767.46404 & {$\left[\left(\mathrm{C}_{2} \mathrm{H}_{4} \mathrm{O}\right)_{17}+\mathrm{H}_{2} \mathrm{O}+\mathrm{H}\right]^{+}$} \\
\hline 811.49025 & {$\left[\left(\mathrm{C}_{2} \mathrm{H}_{4} \mathrm{O}\right)_{18}+\mathrm{H}_{2} \mathrm{O}+\mathrm{H}\right]^{+}$} \\
\hline 855.51647 & {$\left[\left(\mathrm{C}_{2} \mathrm{H}_{4} \mathrm{O}\right)_{19}+\mathrm{H}_{2} \mathrm{O}+\mathrm{H}\right]^{+}$} \\
\hline 899.54268 & {$\left[\left(\mathrm{C}_{2} \mathrm{H}_{4} \mathrm{O}\right)_{20}+\mathrm{H}_{2} \mathrm{O}+\mathrm{H}\right]^{+}$} \\
\hline 943.56890 & {$\left[\left(\mathrm{C}_{2} \mathrm{H}_{4} \mathrm{O}\right)_{21}+\mathrm{H}_{2} \mathrm{O}+\mathrm{H}\right]^{+}$} \\
\hline 987.59511 & {$\left[\left(\mathrm{C}_{2} \mathrm{H}_{4} \mathrm{O}\right)_{22}+\mathrm{H}_{2} \mathrm{O}+\mathrm{H}\right]^{+}$} \\
\hline 1031.62132 & {$\left[\left(\mathrm{C}_{2} \mathrm{H}_{4} \mathrm{O}\right)_{23}+\mathrm{H}_{2} \mathrm{O}+\mathrm{H}\right]^{+}$} \\
\hline 1075.64754 & {$\left[\left(\mathrm{C}_{2} \mathrm{H}_{4} \mathrm{O}\right)_{24}+\mathrm{H}_{2} \mathrm{O}+\mathrm{H}\right]^{+}$} \\
\hline 1119.67375 & {$\left[\left(\mathrm{C}_{2} \mathrm{H}_{4} \mathrm{O}\right)_{25}+\mathrm{H}_{2} \mathrm{O}+\mathrm{H}\right]^{+}$} \\
\hline
\end{tabular}




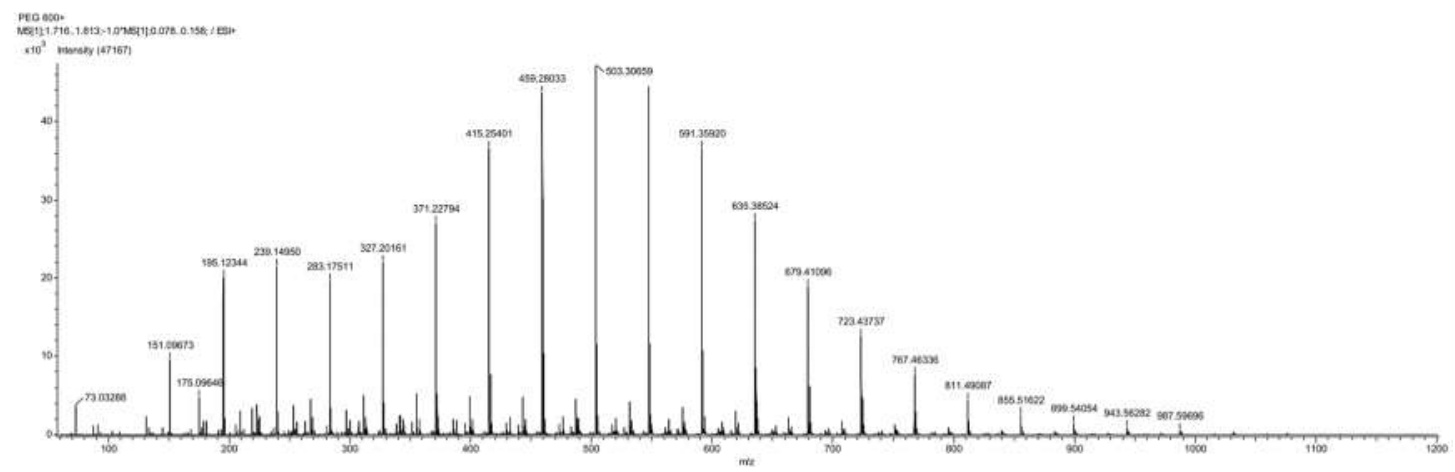

Figure 23 - PEG 600 positive ion mode reference mass spectrum [90].

An example of a typical DART Reconstructed Ion Current (RIC) profile can be seen in Figure 24. Eighteen samples were run in less than eight minutes. The first three peaks correspond to the calibrant, neat polyethylene glycol with an average molecular weight of 600 (PEG 600), and then the sample was sampled in triplicate varying the gas stream temperature throughout the run.

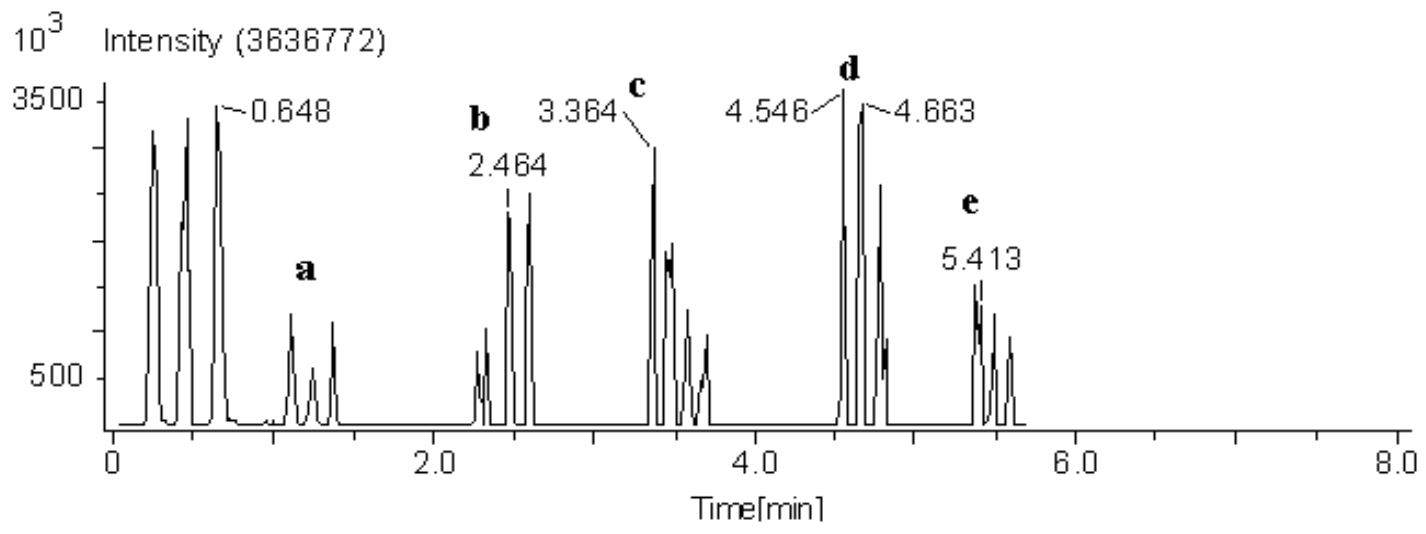

Figure 24 - Total ion response of nylon $6 / 9$; (a) $200^{\circ} \mathrm{C}$, (b) $225^{\circ} \mathrm{C}$, (c) $250^{\circ} \mathrm{C}$, (d) $275^{\circ} \mathrm{C}$ and (e) $300^{\circ} \mathrm{C}$.

Figure 25 and Figure 26 illustrate the importance of function switching, in how it is used to discriminate between closely related chemicals using collision induced dissociation. Both nylon 6 and $6 / 6$ have a major peak at $\mathrm{m} / \mathrm{z} 227.1759$, the difference being 
the fragment ions produced as the orifice 1 voltage is increased. Figure 27 shows the SearchFromList result on the mass spectrum.
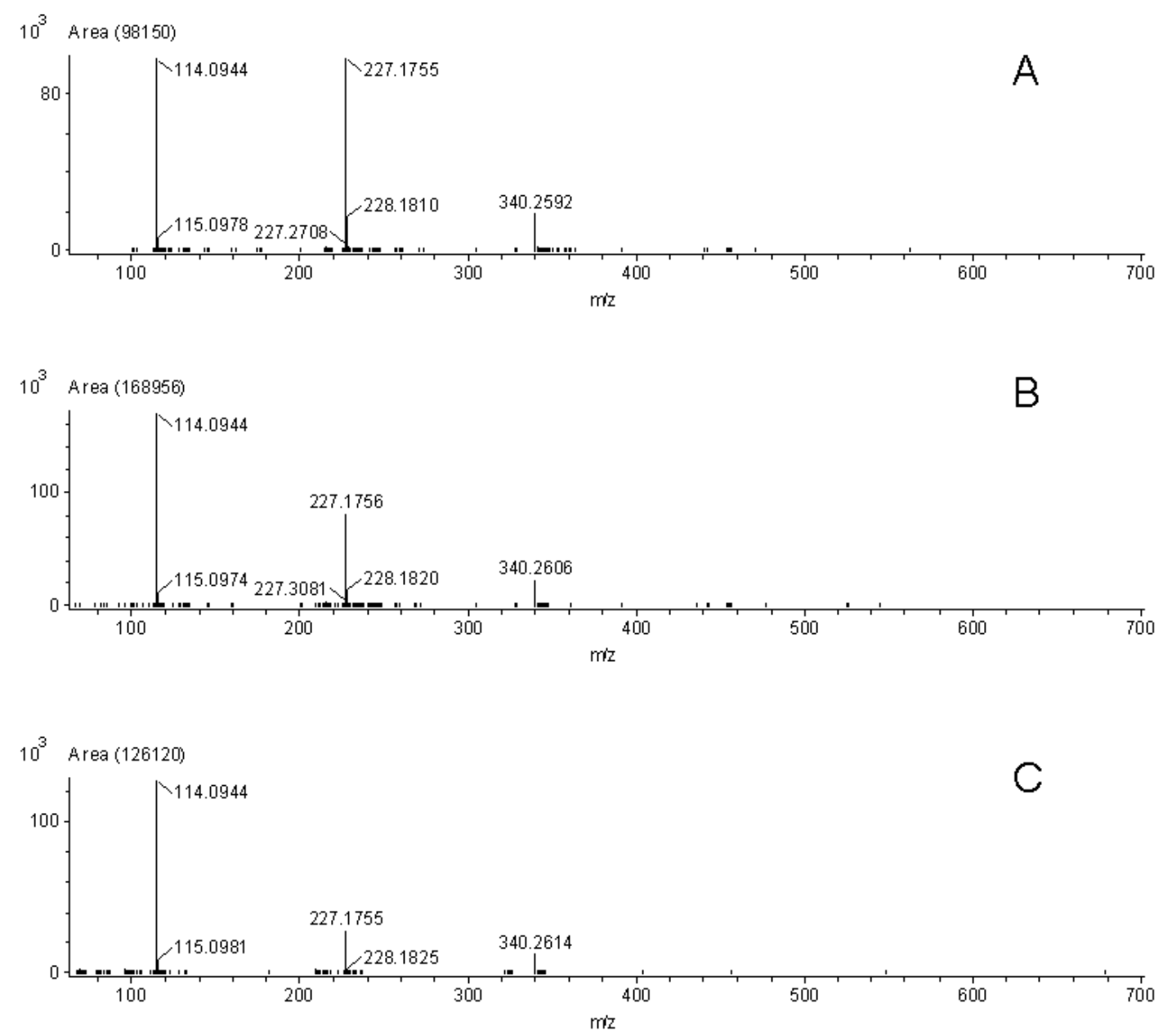

Figure 25 - DART-AccuTOF ${ }^{\mathrm{TM}}$ mass spectra for nylon 6 at orifice voltage of (a) 20 volts, (b) 30 volts and (c) 60 volts. 

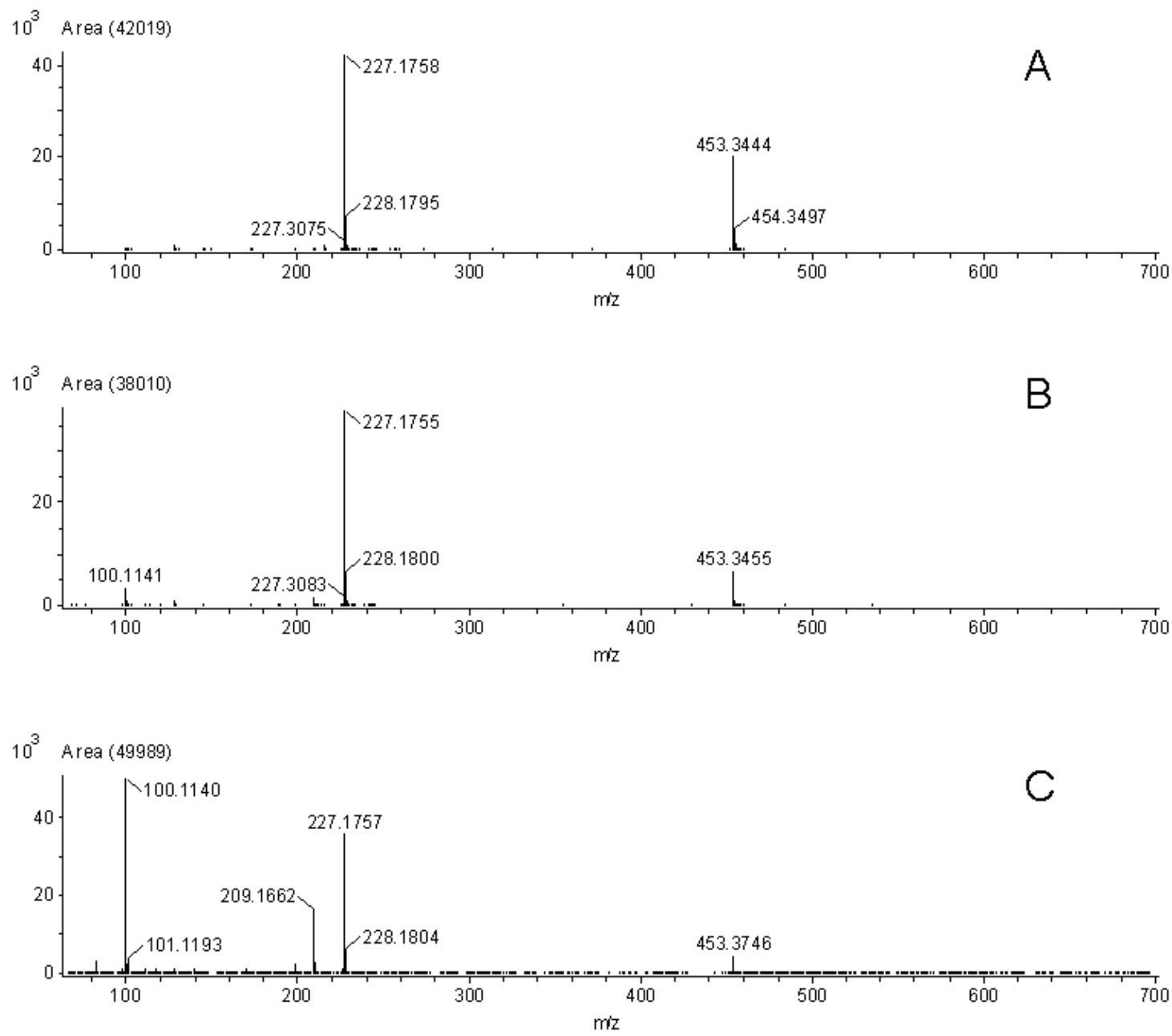

Figure 26 - DART-AccuTOF ${ }^{\mathrm{TM}}$ mass spectra for nylon 6/6 at orifice voltage of (a) 20 volts, (b) 30 volts and (c) 60 volts.

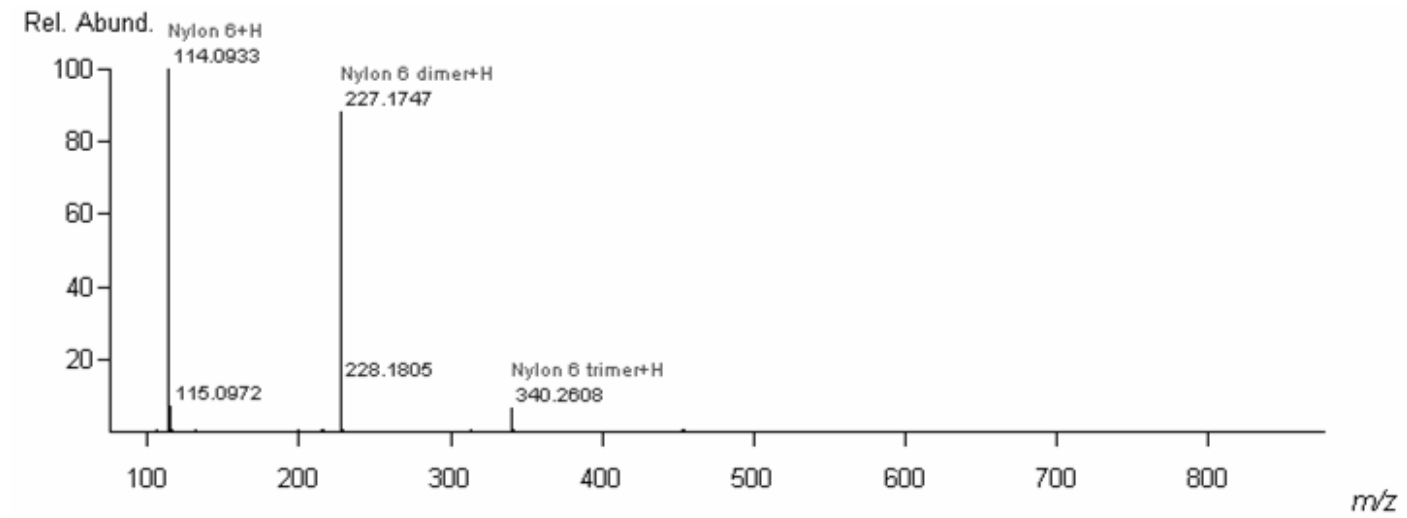

Figure 27 - DART-AccuTOF ${ }^{\text {TM }}$ mass spectra for standard nylon 6 at 20 volts with correct peaks identified using SearchFromList software. 


\subsection{Research Objectives}

The major objectives the present research attempts to expand upon is in regards to the reasons why canines often alert at objects or areas that do not contain any illicit substance that would warrant such an alert. Two underlying phenomena that are hypothesized to play a role in those unconfirmed alerts are (1) the transport of odorants from said illicit substances to areas that do not contain those drugs or explosives in a relatively short amount of time, and (2) residual odors that remain in an area after an illicit substance was briefly present in that location.

By using DART-AccuTOF, these hypotheses can be tested as a result of the instrument's ability to sample headspace and in real time, without the need for preconcentration or prior sample preparation. By first optimizing parameters for the instrument, the odorants that are known to emit from commonly encountered illicit substances in law enforcement are tested. Two explosive odorants and two narcotic odorants will be used for this research. The odorants will be selected during the first task involving parameter optimization.

The second task focused on the transport of odorants by measuring the amount of time it takes for the instrument to detect the volatile compounds from the moment they were exposed, and repeating this process at various distances. If it was determined that these odors were floating around in relatively short amounts of time and at detectable levels to the instrument, it would help explain why canines, through no fault of their own, would falsely alert to an object that did not contain a substance of interest, but instead was in close proximity to a box or object that did contain that substance. 
The third task's objective was to explore the amount of time residual odors needed to persist on various substrate types. If they do in fact persist in relatively short amounts of time, it should be addressed in canine certification guidelines to prevent or reduce the possibility of unconfirmed alerts. By imploring different substrate types, it could also help improve guidelines by either avoiding substrates that easily attract and hold onto these odorants, while also promoting substrate types that are found to do the opposite.

The IFRI training aid kits (COMPS) were tested externally to determine whether any cross-contamination between training aids could occur during storage. Because of the DART's ability to detect in real time, it could theoretically detect odorants escaping the training aids. If cross-contamination is observed, that would mean IFRI would need to either modify the storage material or the manner in which they are stored.

Finally, a survey was to be conducted to obtain information and opinions from canine handlers about what they themselves believe are factors that could affect unconfirmed alerts. By also asking them their opinions on other factors pertaining to canine certification, their responses could help either adjust guidelines or rewrite them.

Overall, by researching why canines sometimes falsely alert to objects or areas, it could help the canine handling and training community improve the quality of their work by eliminating or substantially reducing the number of unconfirmed alerts. The findings of this research could also help prevent canines and their handlers failing their certifications through no fault of their own, but simply due to a flaw in how the certification was set-up or conducted. 


\section{MATERIALS AND METHODS:}

\subsection{Materials}

\subsubsection{Chemicals}

- Methyl Benzoate

- Sigma Aldrich M29908 - 500g. Lot \# STBF7480V

- Benzaldehyde

○ Sigma Aldrich 418099 - 100mL. Lot \# SHBG1438V

- 2-ethyl-1-hexanol

○ Sigma Aldrich 538051 - 500mL. Lot \# MKBP6498V

- 2,4-dinitrotoluene

○ Sigma Aldrich D200603, Lot \# 19189TA

- Diphenylamine

○ Sigma Aldrich 112763 - 100g. Lot \# MKBV3210V

- Diethyl Phthalate

○ Sigma Aldrich 524972 - 100mL Lot \# MKBJ3578V

- Dibutyl Phthalate

○ Sigma Aldrich 524980 - 100mL. Lot \# MKBR4111V

- Ethyl Centralite (1,3-Diethyl-1,3-diphenyl-urea)

○ Sigma Aldrich 372889 - 100g. Lot \# 05107LFV

- Methyl Centralite

○ Sigma Aldrich 372889-100g. Lot \# 05107LFV 
- Piperonal

○ Sigma Aldrich P49104-100G, Lot \# MKBS7607V

- 2,3-Dimethyl-2,3-Dinitrobutane

○ Sigma 156345 - 50g. Lot \#MKBT8831V

- Polyethylene glycol (PEG 600)

○ Ultra Scientific 025322-68-3 10mL, Lot \# RM00390

\subsubsection{IFRI COMPS}

- Explosive Mimics

- Blank Explosive Training Aid Mimic \#1 (Lot \# 101116-01)

- Blank Explosive Training Aid Mimic \#2 (Lot \# 101116-01)

- Nitroglycerin Explosive Training Aid Mimic (Lot \#10112016-01)

- TNT Explosive Training Aid Mimic (Lot \# 101116-01)

- Plasticized Explosive Training Aid Mimic (Lot \# 101116-01)

- Smokeless Powder Training Aid Mimic \#1 (Lot \# 101116-01)

- Smokeless Powder Training Aid Mimic \#2 (Lot \# 101116-01)

- Narcotic Mimics

- Cocaine Training Aid (Lot \# VSF101116-01)

- Methamphetamine Training Aid (Lot \# VSF101116-01)

○ MDMA Training Aid (Lot \# VSF101116-01)

\subsubsection{Other Supplies}

- Analytical balance

○ Melter Toledo XP205 
- Top-loading balance

- Melter Toledo SB 32000

- Heat seal

○ Midwest Pacific

- Disposable weight boats (2.5”, and 4")

○ Fischer 08-732-117

- Cellulose

- Sigma Aldrich 310697-1KG, Lot \# MKBX5118V

- Alumina Powder

○ Fischer A540-500, Lot \# 162621

- $100 \mathrm{~mL}$ beaker

○ Pyrex Inc ${ }^{\circledR}$

- $20 \mathrm{~mL}$ Disposable Scintillation Vials

○ Article\#: FS740504-20, Lot \#: 3-4A-18

- Mortar \& pestle

- Ball Canning Jars

- Publix, 8oz regular mouth 2 piece lid

- Parafilm

- Bemis Flexible Packaging PM992

- Barrier foil ziplock bags (6" x 5.5" and 8" x 10.5" x 3.5")

- Ted Pella, Inc. 139-310 and 139-313

- Low-density polyethylene (LDPE) Polyethylene bags 3" x 3" (38- 2mil LDPE) 
○ \#ITLD355, 3x3 2mil clear LDPE 2000/cs

- Gauze pads (20-2” x 2" 8 ply sterile sponges)

○ 2"x2" 8 ply, 50 pack/box

- Disposable Nitrile Exam Gloves:

○ Size XL

○ Lot No: 508DD224

- Winchester 760 Smokeless Powder

○ Winchester Repeating Arms Company, New Haven, CT

- Hodgdon H 4895 Smokeless Powder

○ Hodgdon Powder Company, Shawnee, KS

- Laser measure

○ Bosch GLM 40

○ C Robert Bosch Tool Corporation Lincolnton, NC

- Wood Toothpicks:

○ YourHome Brand, Harris Teeter

- Metal paperclips

- WOW OfficeWorks, Inter-American Products, Cincinnati, OH

- Glass Capillary Tubes

○ Pyrex $®$ Part No: 9530-02

○ $\quad 0.8-1.1 \times 100 \mathrm{~mm}$

- Fisherbrand ${ }^{\circledR}$ Petri Dishes:

- Fisher Scientific

o Cat No.: 09-720-500 
○ Lot No: F5JA15386

- Sterilite ${ }^{\circledR}$ Plastic Container with Lid:

○ C 2014 Sterilite Corporation, Townsend, MA 01469

○ $\quad 62.5 \mathrm{~cm} \mathrm{~L} \mathrm{x} 47.6 \mathrm{~cm} \mathrm{~W} \mathrm{x} 17.5 \mathrm{~cm} \mathrm{H}$

- Gladware ${ }^{\circledR}$ Plastic Containers with Interlocking Lid:

○ (C) The Glad Products Company, Oakland, CA

○ $17.5 \mathrm{~cm} \times 17.3 \mathrm{~cm} \times 12.2 \mathrm{~cm} ; 7.2$ ounces

- Elite Aura Ductless Fume Hood:

- Mystaire $^{\mathrm{TM}}$, Creedmoor, NC

- Model \#: MY-AU42

○ Serial \#: MYAU421-254

\subsection{Instrumentation}

\subsubsection{Direct Analysis in Real Time - Accurate Time of Flight Mass Spectrometer}

- DART - AccuTOFTM (JEOL USA, Inc., Peabody, MA, USA)

- Analyzer Method

- Ion Guide Peak Voltage: 400V

- Ion Guide Bias Voltage: 27V

- Focus Voltage: $-120 \mathrm{~V}$

- Condenser Lens Voltage: 10V

- Quadrupole Lens Voltage: 20V

- Right/Left Lens Voltage: $-4 \mathrm{~V}$

- Top/Bottom Lens Voltage: $-0.1 \mathrm{~V}$ 
- Pusher Bias Voltage: $-0.35 \mathrm{~V}$

- Reflectron Voltage: 900V

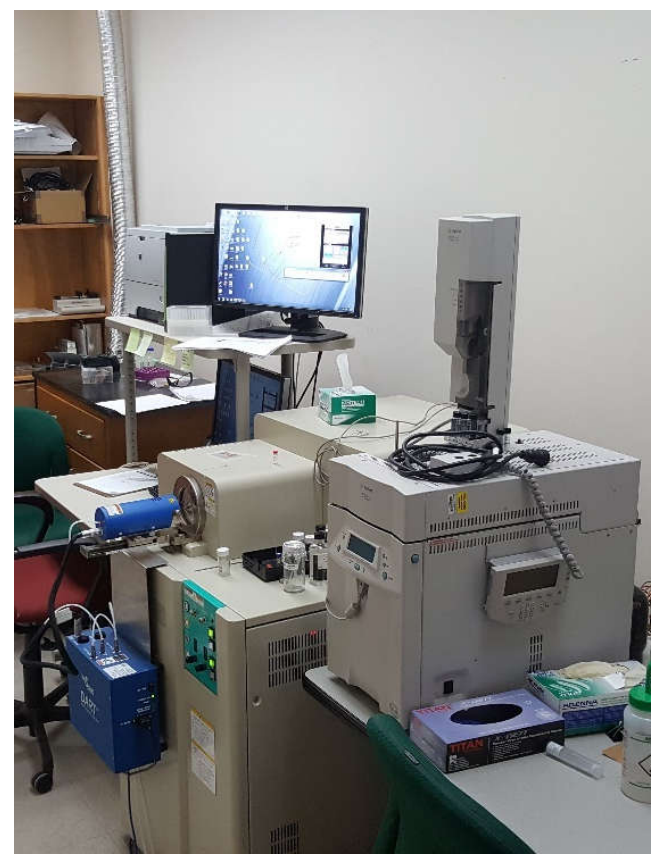

Figure 28 - DART-AccuTOFTM at the Virginia Commonwealth University laboratory.

- Analyzer (Advanced) Method

- Pusher Voltage: $778 \mathrm{~V}$

- Pulling Voltage: $-778 \mathrm{~V}$

- Suppress Voltage: $0.00 \mathrm{~V}$

- Flight Tube Voltage: -7000

○ Detector Method:

- Detector Voltage: $2000 \mathrm{~V}$

○ Ion Source Method

- Needle Voltage: 2000V

- Peaks Voltage: 2500V 
- Orifice 1 Voltage: 20, 30, 60 and 90V used.

- Orifice 2 Voltage: $5 \mathrm{~V}$

- Ring Lens Voltage: 10

- Orifice 1 Temp: $80^{\circ} \mathrm{C}$

○ Spectrum Monitoring

- Acquisition Range: $40-1000$ m/z

- Spectrum Recording Interval: $1.0 \mathrm{~s}$

- Wait Time: 0.030s

- Data Sampling Interval: 0.5ns

- Update Interval: 1.0s

- X-axis Unit of Chart: $\mathrm{m} / \mathrm{z}$

- Calculation Method of Peak Position: FWHM

- Calculation of Peak Position: Top

- Correct Baseline: No

- Perform Smoothing: No

- DART Parameters:

- Gas Temperature: $150^{\circ} \mathrm{C}-500^{\circ} \mathrm{C}$

- Grid Voltage: 200V (Positive mode)

- Standby Gas: Nitrogen

- Run Gas: Helium

- Sample Gap: 10 mm 
- Software:

- DART software:

- DART SVP:

- Software version: 4.5.5

- Firmware version: 2.3.3

○ MassCenter Main:

- Version 1.3.0.1

- C JEOL Ltd.

- MassCenter System:

- Version 1.5.0k

- C JEOL Ltd.

○ MSTune Manager:

- Version: 1.3.0.0

- TSS Pro:

- Version 3.0

- (C) Shrader Analytical and Consulting Laboratories, Inc.

○ Mass Mountaineer:

- RBC Software. Mass spectral interpretation tools.

- C R.B. Cody 2012

- Version 3.0 
Data are collected and analyzed using JEOL MassCenter software version 1.3.0.1. All data are collected in profile or continuous data mode. The mass spectra are “centroided", calibrated, then saved as a text delimited JEOL-DX File (*.jsp).

In addition to this software, another program was developed called Mass Mountaineer by R.B. Cody, which helps by searching against a spreadsheet containing the neutral mass composition of various chemicals. By selecting the appropriate adduct, which in most cases is a proton, the software calculates the exact mass of each protonated molecule in the spectrum and matches it with the corresponding molecule with an exact mass within $5 \mathrm{mDa}$.

\subsection{Methodology}

\subsubsection{Task 1: Optimizing DART Parameters and Identification of Chemical Standards to be Used.}

The first task involved optimizing instrumental parameters. The vast majority of parameters were untouched and the values used followed the manufacturers recommendation as shown in Section 3.1.1. The two parameters that require samplespecific or method-specific adjustments are (1) temperature of the DART stream and (2) the orifice 1 voltage of the mass spectrometer. Beginning with the orifice 1 voltage, the manufacturer recommends using lower voltages $(20-30 \mathrm{~V})$ as it will be sufficient for most needs. However, there were instances where higher voltages were used to promote collision induced dissociation to help create fragment ions that could help further and it will be discussed in the Results section. Changing the orifice 1 voltage is done using MS Tune Manager, under the settings pane as shown in Figure 29. 


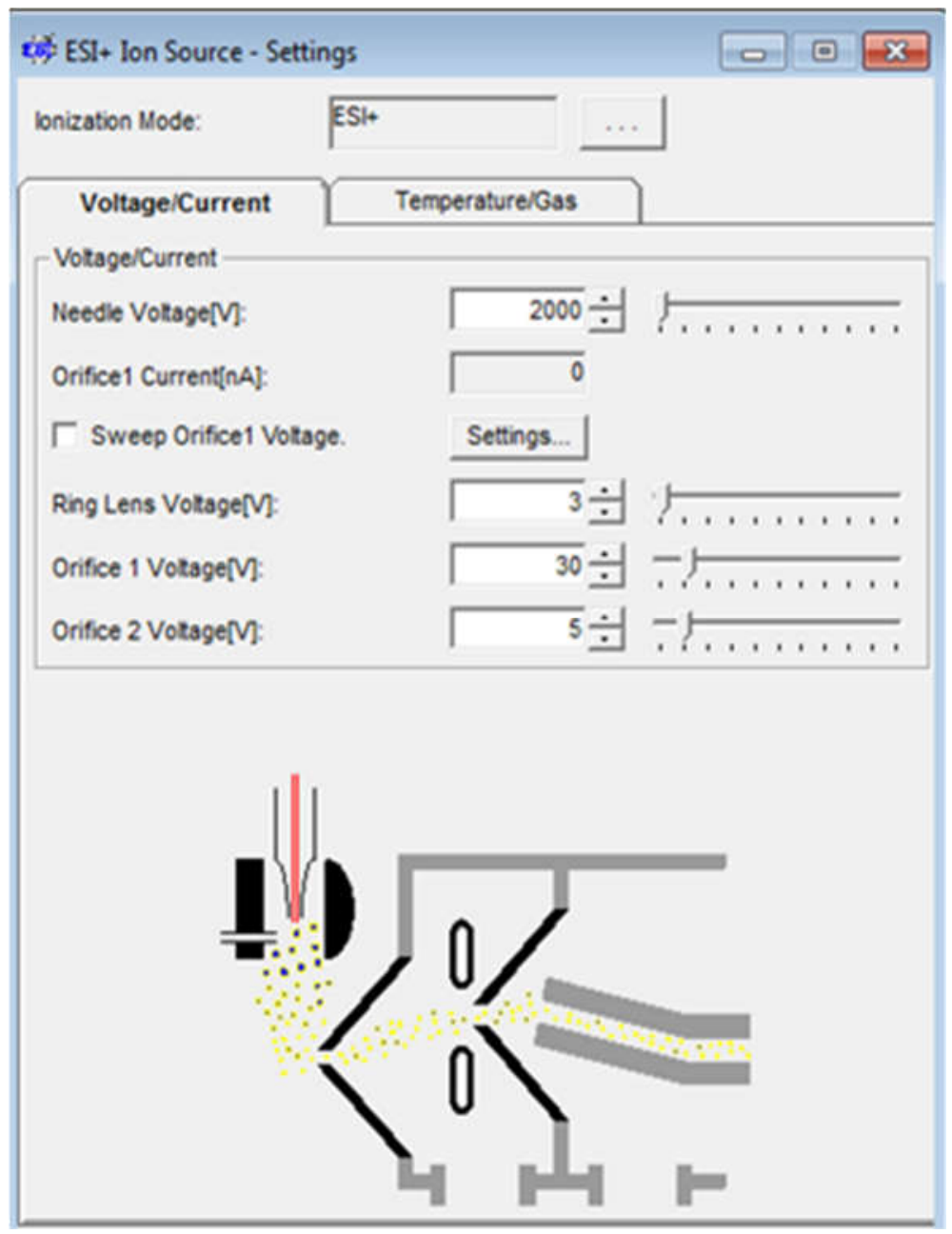

Figure 29 - Settings pane in MS Tune Manager, where orifice 1 voltage, among other parameters, can be chosen.

For temperature, the chemical standards were tested at temperatures from $150^{\circ} \mathrm{C}$ to $500^{\circ} \mathrm{C}$ at $50^{\circ}$ intervals. In order for the calibrant (PEG 600) to be analyzed properly, each run must begin at a temperature of either $300^{\circ} \mathrm{C}$ or $350^{\circ} \mathrm{C}$, and the sampling of the PEG calibrant is done in triplicate. Therefore, when the run starts at $300^{\circ} \mathrm{C}$, the temperature is ramped down $50^{\circ} \mathrm{C}$ for the next sample, until $150^{\circ} \mathrm{C}$, and when it starts at $350^{\circ} \mathrm{C}$, it is ramped up $50^{\circ} \mathrm{C}$ for the following sample, until it is sampled at $500^{\circ} \mathrm{C}$. Temperature of the DART stream is controlled using the DART SVP software. 


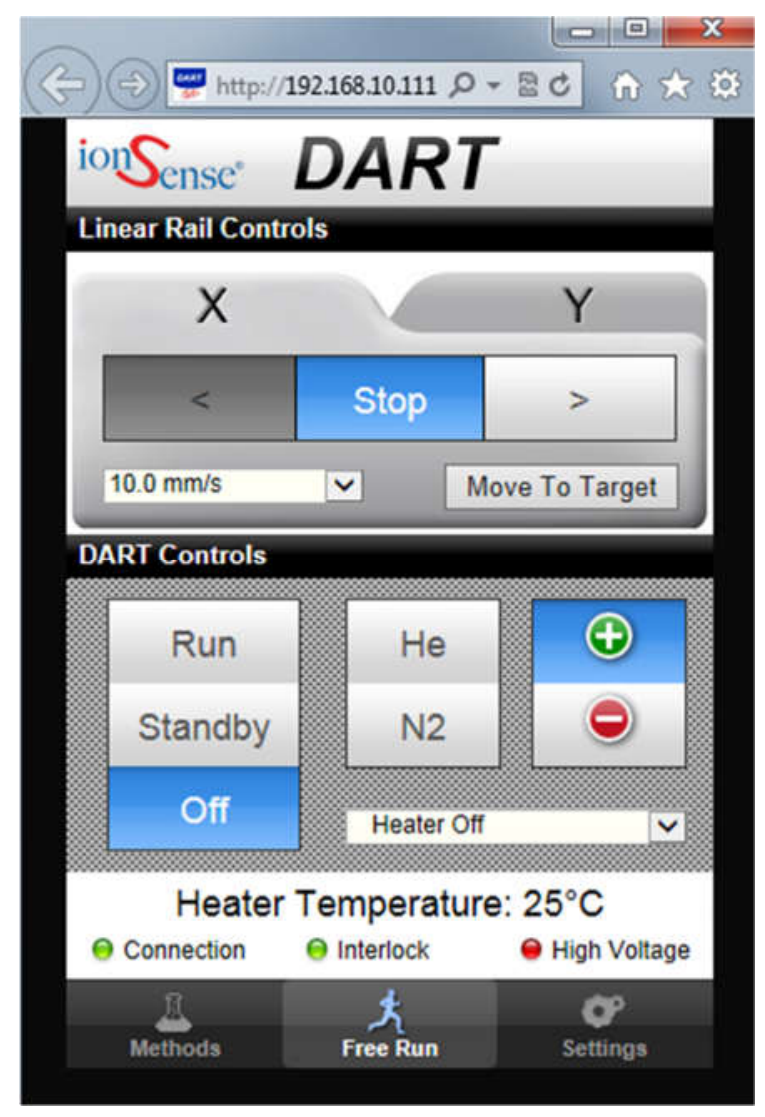

Figure 30 - DART SVP software. Temperature can be chosen in $50^{\circ} \mathrm{C}$ intervals from the dropdown menu where "Heater Off" is located.

Approximately $5 \mathrm{ml}$ of each chemical standard used in this task was stored in a $20 \mathrm{~mL}$ disposable scintillation vial. When sampling, the cap is removed and the headspace is introduced near the DART stream for ionization (Figure 31). For each standard, the headspace of each chemical was sampled in triplicate at each temperature interval. This creates 15 samples per run (First 3 are PEG at temperature $X$, then 3 samples of the standard at temperature $\mathrm{X}$, then 3 samples of the standard at temperature $\mathrm{X}+/-50^{\circ} \mathrm{C}$, etc.). Not only was each sample done in triplicate, but each run was done in triplicate as well. This creates 9 data points for each chemical standard at each temperature interval. 


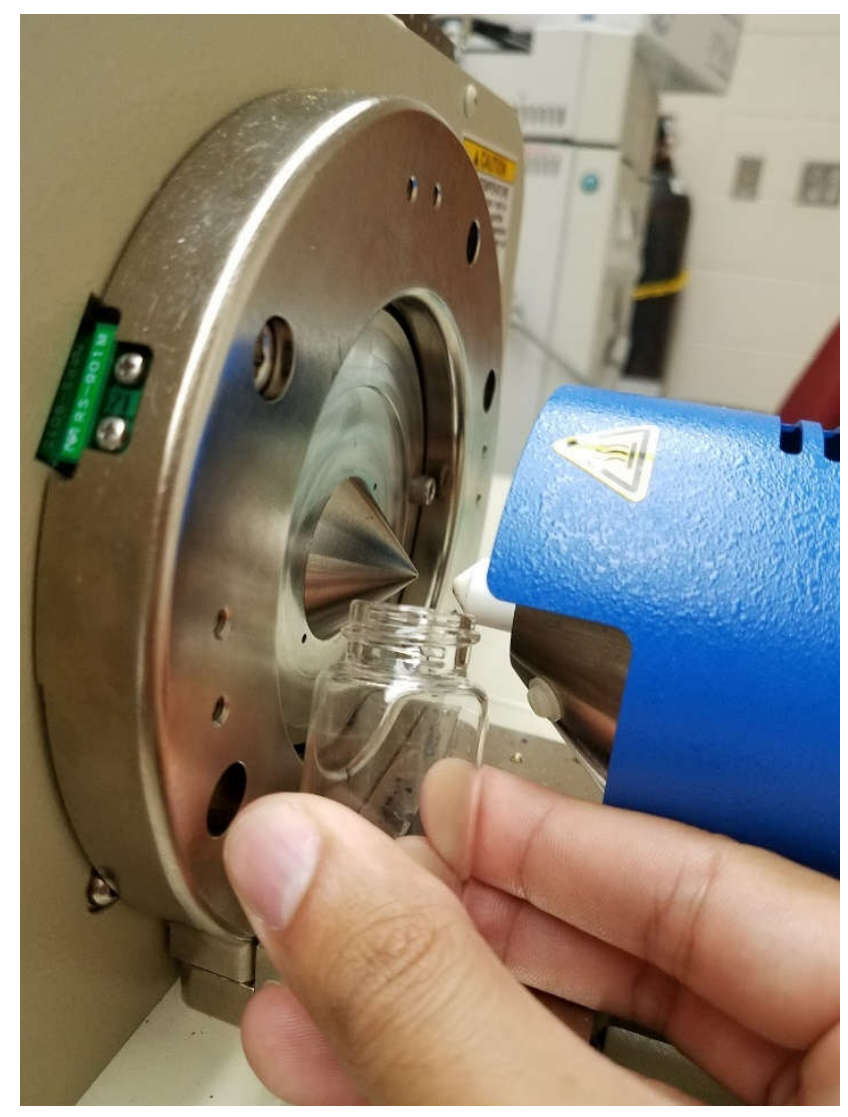

Figure 31 - Manner in which headspace of liquid samples are analyzed using the DART.

Eight chemical standards (methyl benzoate, benzaldehyde, 2,4-dinitrotoluene, 2ethyl-1-hexanol, diphenylamine, diethyl phthalate, dibutyl phthalate, 1,3-Diethyl-1,3diphenyl-urea), were tested during this task, and the objective was to narrow it down to four standards for the remainder of the research (two narcotic odors and two explosive). The choices are explained in the Results section.

Data analysis begins first with 'translating' the data file in TSS Pro 3.0. Before calibration, the area in the RIC prior to the PEG peaks are selected using the right mouse click, and 'perform background subtraction' is chosen. Next, there is a button right above the RIC that will perform 'CODA' (Component Detection Algorithm), which is a method that extracts high quality RIC profiles from more complex data sets[91] Next, the PEG 
peaks are averaged and is then used to calibrate the entire data file. Following calibration, each peak in the RIC is averaged to create a mass spectrum and the intensity of each peak was recorded in a spreadsheet. This process is repeated for every run and every sample. For the software to correctly identify each peak in the mass spectrum, the $\mathrm{m} / \mathrm{z}$ must be within $\pm 5 \mathrm{mDa}$ and the peak itself must be at a minimum of $5 \%$ of the relative intensity. Anything below 5\% would be omitted, and anything outside the $\pm 5 \mathrm{mDa}$ range would not be used.

\subsubsection{Task 2: Evaluating the Speed of VOC Transport}

Every run in this task used the parameters identified as being optimal from Task 1 for analysis. Also, the four chemical standards identified following the completion of Task 1 were used to complete this experiment.

Each of the four chemical standards chosen were sampled at five various distances $(0.5 \mathrm{~m}, 1 \mathrm{~m}, 1.5 \mathrm{~m}, 2 \mathrm{~m}, 3 \mathrm{~m})$. In Figure 32 , the layout of the room this experiment was done in is drawn to illustrate where the samples were held relative to the DART source. The sampling was done along the red line at the aforementioned distances. The objective is to determine how long it takes for the VOCs of each sample to be picked up by the instrument at these various distances. Therefore, this involved the use of a timer to mark the point in which the cap of the vial was opened during each run. 


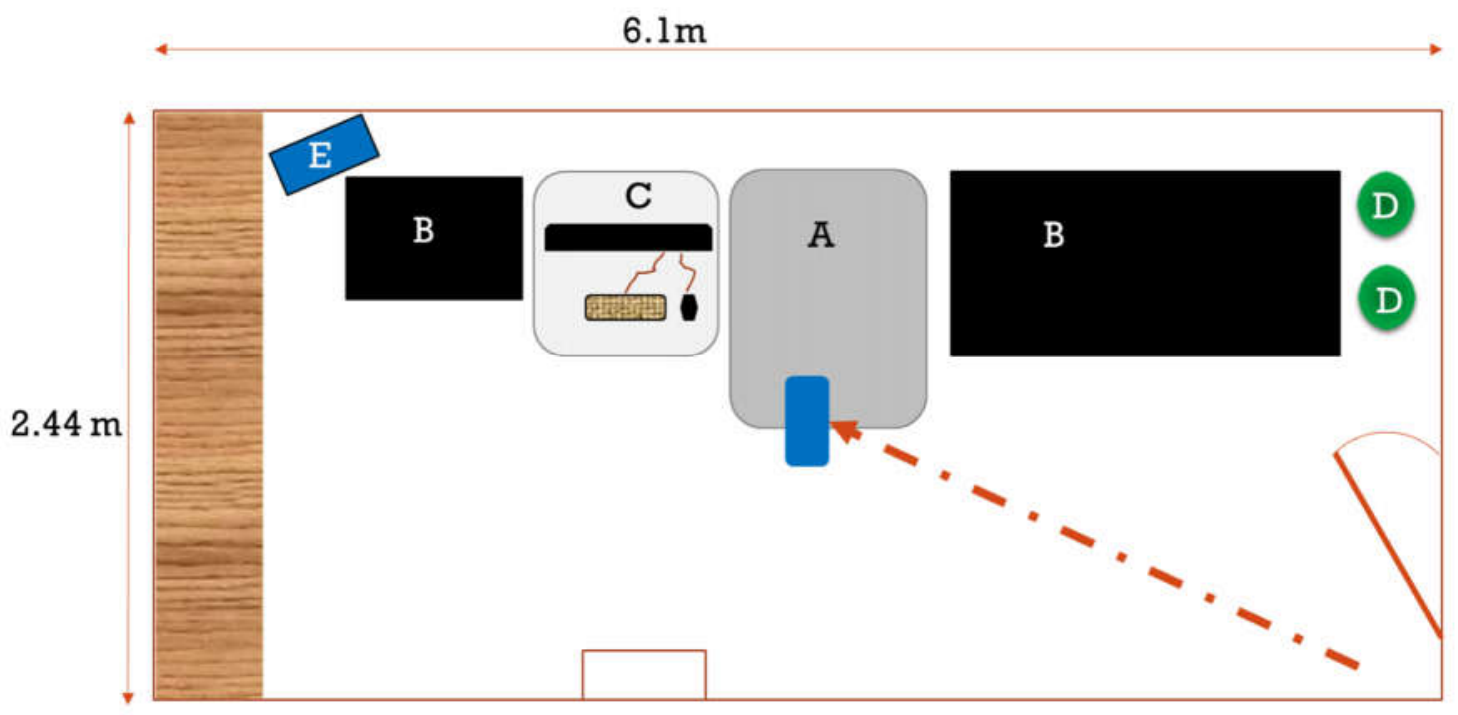

Figure 32 - Layout of the room where the DART-AccuTOFTM (A) is located. Where B are desks, $\mathrm{C}$ is the computer desk, $\mathrm{D}$ are the tanks, and $\mathrm{E}$ is an air conditioning unit. (Drawing not to scale)

A typical run would be done as follows:

1. Begin Sample Run + turn timer on simultaneously.

2. Sample PEG calibrant three times.

3. Open vial + press lap on timer to record the moment the vial cap was removed.

4. Keep vial open for 2 minutes.

5. Close vial + press lap to record the moment vial was closed.

6. Open vial once again to sample headspace directly next to DART stream.

7. End run

This was repeated two more times for a total of three runs for each sample at each distance, creating 60 different data points. Step 6 is done to have a positive standard.

When analyzing the data, the same steps done in Task 1 are to be followed until calibration was achieved. Following calibration, the positive standard peak (last peak) in 
the RIC is averaged to create a mass spectrum that will show the molecular ion peak. By right clicking on that peak, the software gives an option to 'create chromatogram(s)', which essentially creates an RIC where only the areas where that peak's $\mathrm{m} / \mathrm{z}$ was identified (Figure 33).

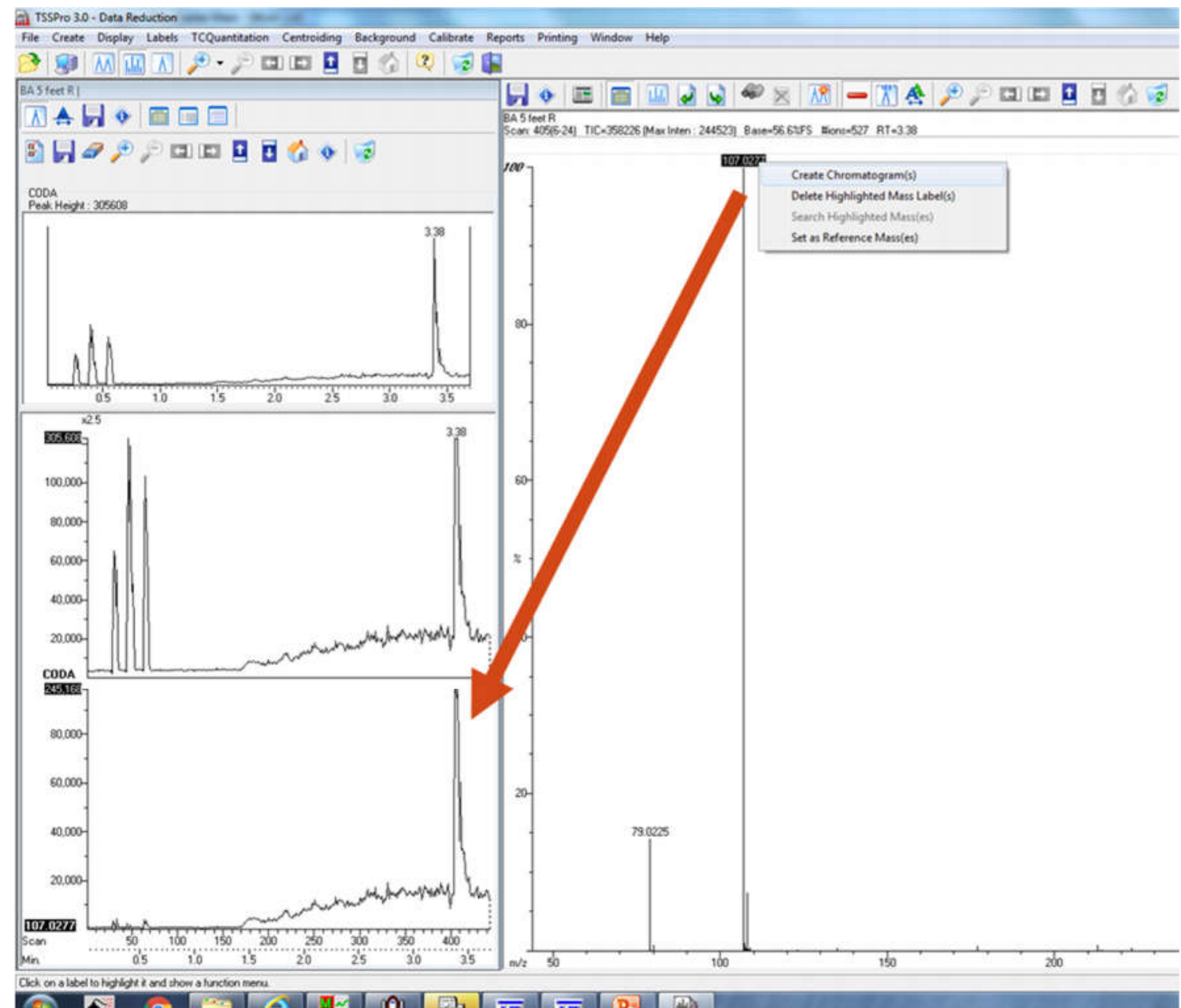

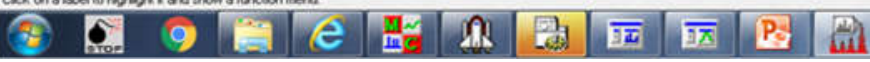

Figure 33 - Example showing the different RIC's. Top RIC shows what the RIC looks like after CODA is performed, while the middle RIC is the before CODA, and the bottom is the RIC created using only the $\mathrm{m} / \mathrm{z}$ information selected from the mass spectrum (right). In this case, the bottom RIC shows the responses where only $107 \mathrm{~m} / \mathrm{z}$ was identified.

Next, the peaks are averaged in the RIC (Figure 34) to create a mass spectrum to try and find the precise moment when the instrument detects the VOC. Once it is properly 
identified, the 'retention time' (RT) shown in the mass spectrum is recorded. It is important to note that RT in this software uses minutes but does not show seconds. Therefore, when each $\mathrm{RT}$ is recorded, it is also converted to seconds in a separate spreadsheet (i.e. $1.75 \mathrm{~m}=$ $105 \mathrm{~s})$.

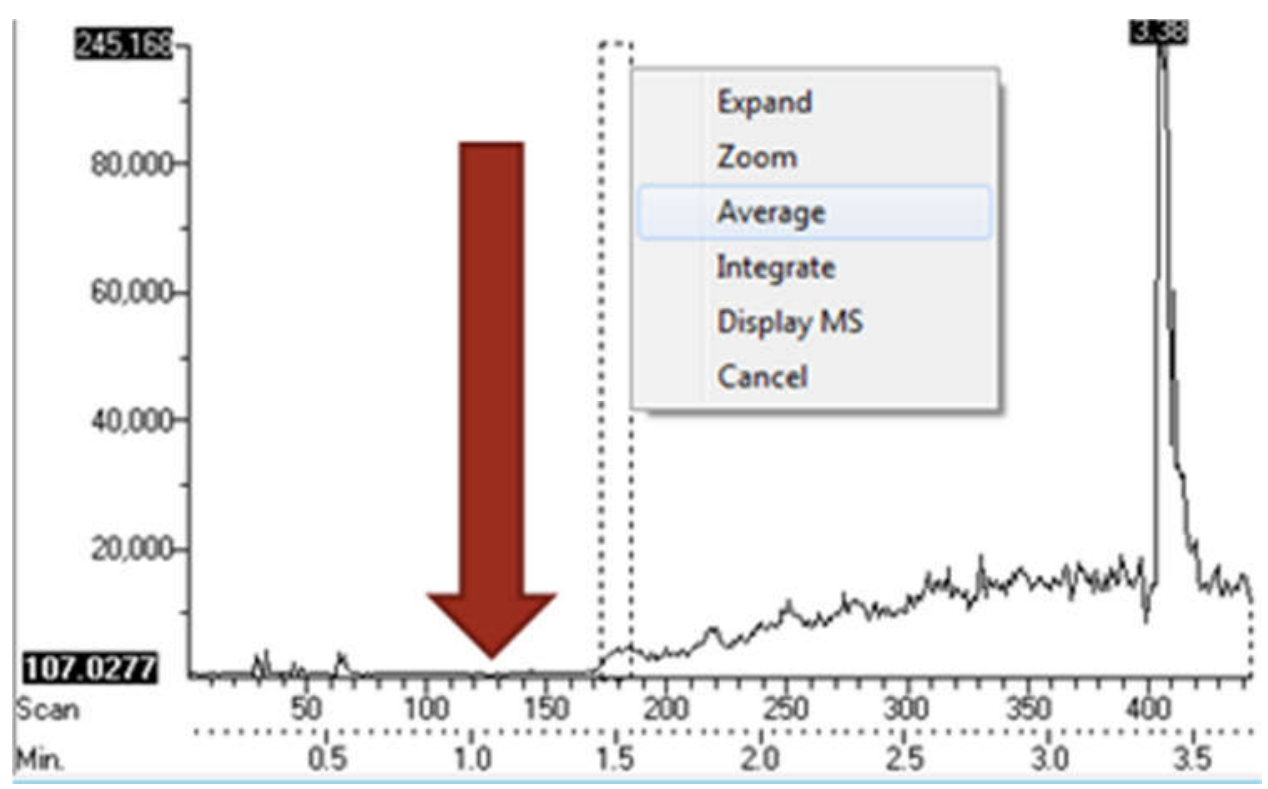

Figure 34 - RIC of $107 \mathrm{~m} / \mathrm{z}$. Red arrow was added by user to indicate the time when the vial was opened. Selected area is the first peak that identified detection of benzaldehyde.

After all the data have been recorded, the amount of time it takes for the odorants to transport at the various distances was calculated (Time VOC was detected minus time vial was opened). For samples that were not successfully detected within that time frame, the exposure time was increased to 5 minutes.

\subsubsection{Task 3: Residual Odor Study}

The third task tries to determine the amount of time needed for VOCs to adhere to a substrate via adsorption, absorption, or other method. The main concern being addressed is whether it is possible for these odors to transport and attach themselves to nearby 
substrates in a relatively short amount of time. The experiments for this task evolved throughout the process, and each of the processes will be described and their results discussed.

First, the substrates used in these experiments were chosen to represent various types of substrates usually encountered at the crime scene. Toothpicks were chosen to replicate wood and clothing, and because of the ease of sampling within the DART stream gap. Glass capillary tubes were chosen to replicate the glass found at crime scenes. Finally, metal paper clips were chosen to replicate the various metals that could be found in a crime scene. It is important to note that the DART does not ionize metals, and, in fact, creates a ‘negative' peak when sampled (Figure 35).

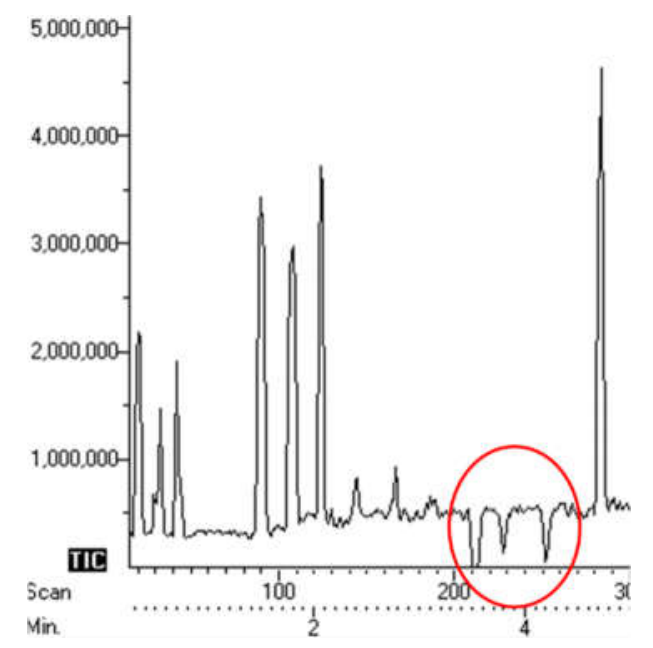

Figure 35 - Example of RIC where metal is ionized, creating a 'negative' peak, which artificially raises the baseline.

\subsubsection{Task 3: Part A}

The first experiment involved the use of a large $(62.5 \mathrm{~cm}$ x $47.6 \mathrm{~cm} \times 17.5 \mathrm{~cm})$ plastic box with a removable lid (Figure 36). The different substrates are placed inside the box and are arranged in a way so that all substrates are at similar distances from the center 
of the box. Next, an empty petri dish is placed in the middle of the box. With the use of a timer, approximately $200 \mu \mathrm{L}$ of the sample of interest (either methyl benzoate, benzaldehyde, 1-ethyl-2-hexanol or diethyl phthalate) is added onto the petri dish, the lid covered and the timer started. Then, the substrates are removed after particular exposure times $(10 \mathrm{~s}, 30 \mathrm{~s}, 1 \mathrm{~m}, 2 \mathrm{~m}, 5 \mathrm{~m}, 10 \mathrm{~m}, 15 \mathrm{~m}, 30 \mathrm{~m}$, and $1 \mathrm{~h})$ and tested immediately on the DART. Each substrate at each exposure time was tested in triplicate. Therefore, there were 108 toothpicks, 108 glass capillary tubes, and 108 metal paper clips used in this experiment for each chemical standard used and for each exposure time.

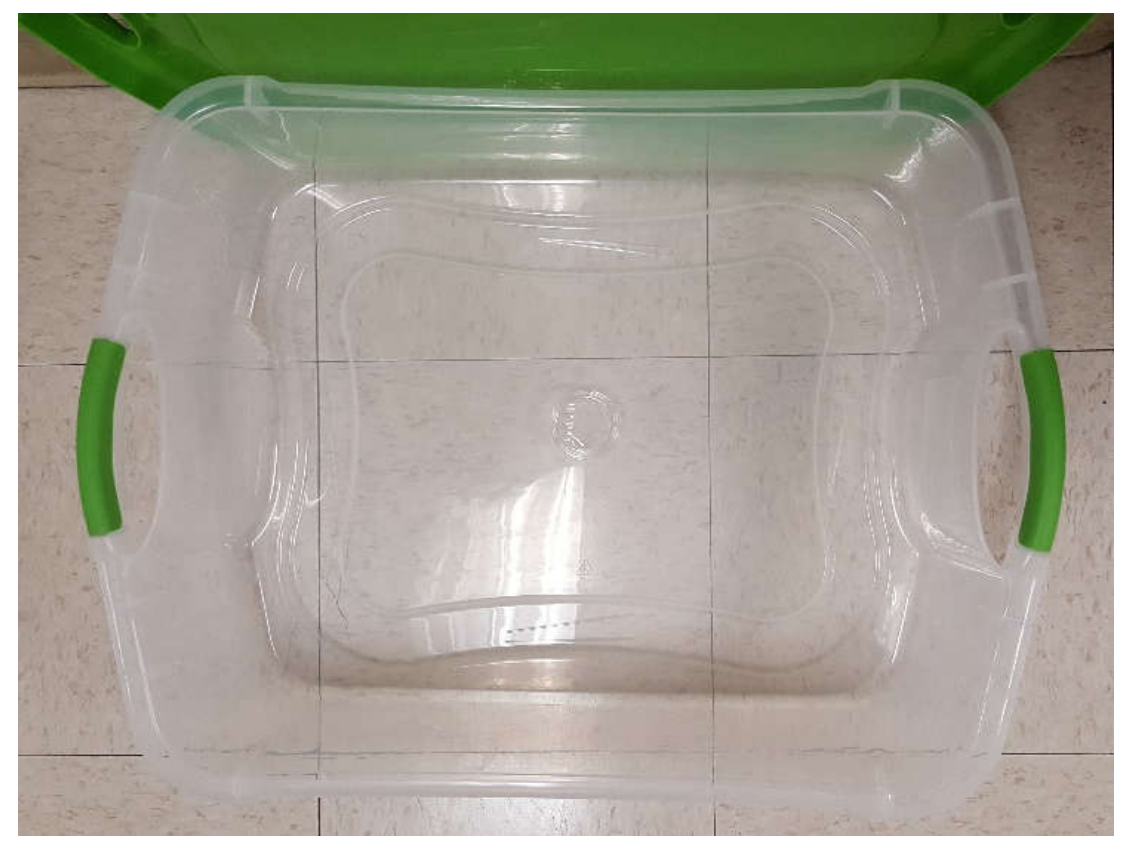

Figure 36 - Picture of the large plastic container used in experiment Task 3, Part A. In the middle, an empty petri dish.

After the testing of the substrates for a given particular chemical standard, that same standard is sampled directly to the DART stream via headspace analysis. This serves the purpose of a positive control, which could later help identify low intensities using the software. 


\subsubsection{Task 3: Part B}

Part B of this task involved a completely different approach. Smaller plastic containers $(17.5 \mathrm{~cm} \times 17.3 \mathrm{~cm} \times 12.2 \mathrm{~cm})$ were used instead, and the method differs as well. For each chemical standard, four plastic containers were used. Within each container, a petri dish containing $200 \mu \mathrm{L}$ of the standard was placed in the middle, and the lids were sealed and the time recorded. Instead of adding the substrates in immediately, the containers were sealed to allow for the area inside to be completely saturated. To ensure saturation, these containers were left untouched overnight.

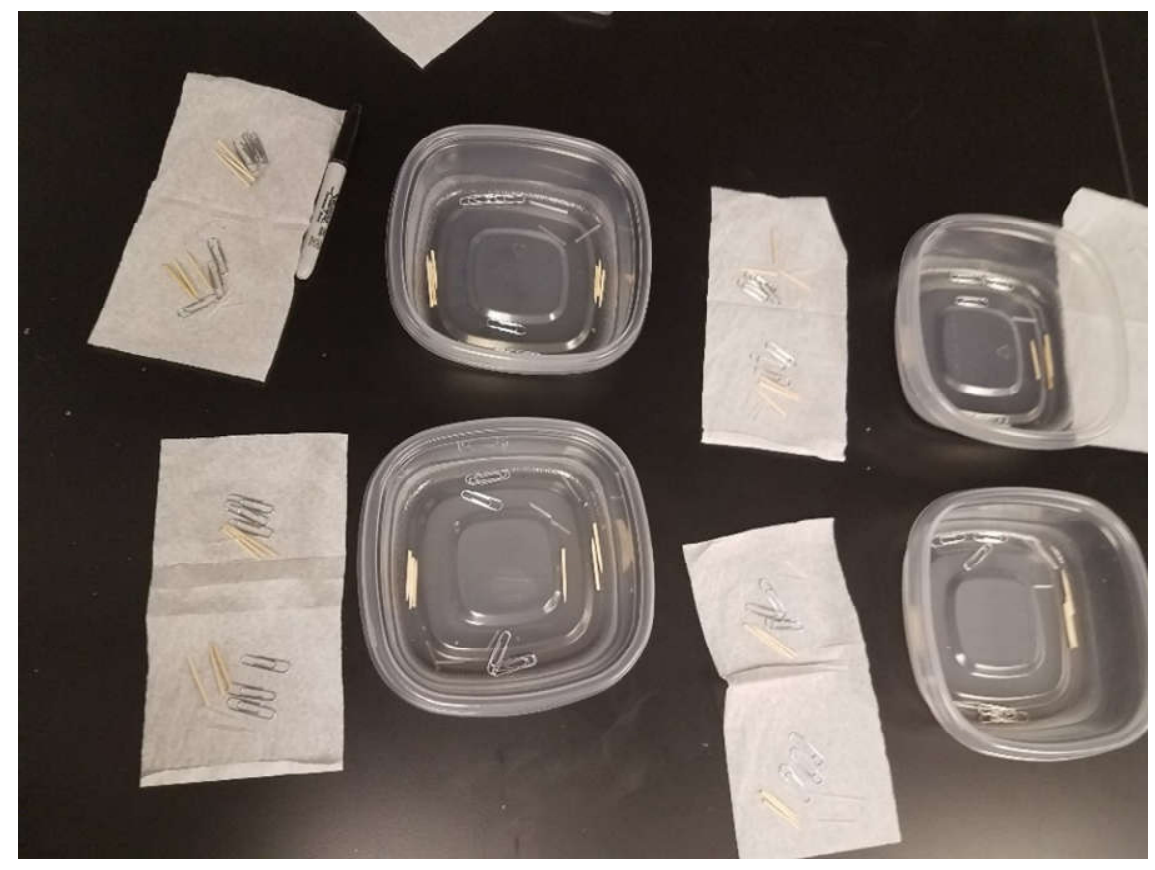

Figure 37 - Example set up for Task 3, Part C. 


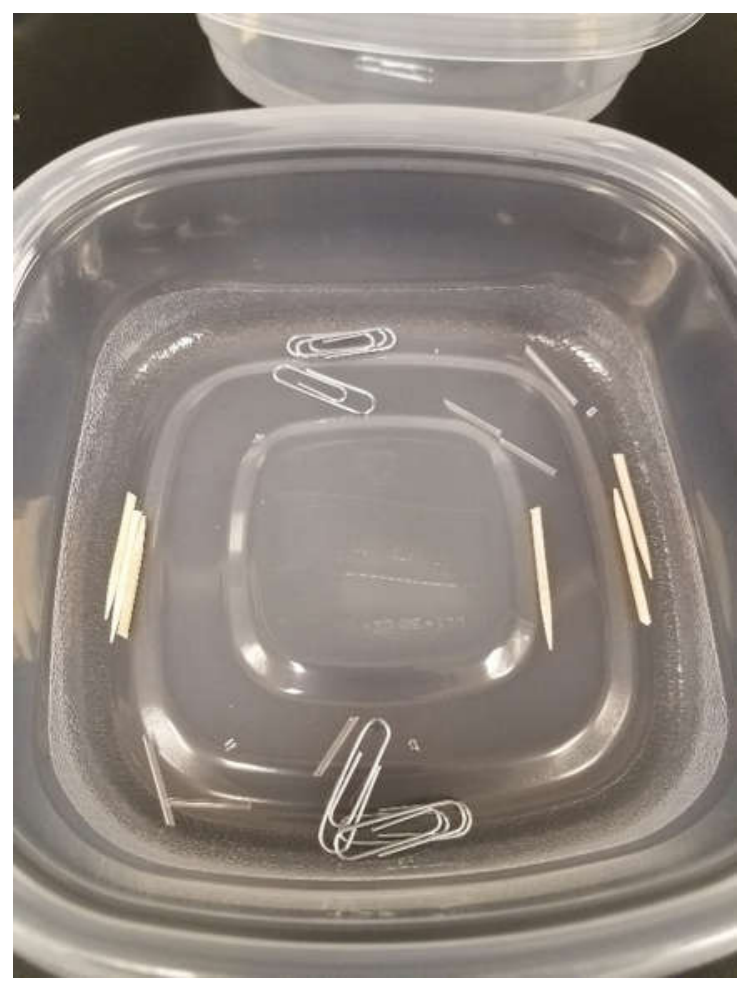

Figure 38 - Example of one of the Glad ${ }^{\circledR}$ plastic containers with 6 toothpicks, 6 glass capillary tubes (broken) and 6 metal clips. The number of substrates indicate that 2 exposure times will be tested using this container ( 3 samples of each substrate for each exposure time).

Following saturation, the lids get slightly opened to allow for the placement of the different substrates within the container. Again, each substrate was tested in triplicate. The four containers were used for four exposure times simultaneously (5m, 15m, 30m and $1 \mathrm{~h})$. After the substrates were exposed to the chemical standard for their corresponding times, the containers were opened and the substrates were tested immediately. In total, 48 toothpicks were used in this experiment, in addition to 48 glass capillary tubes and 48 metal paper clips.

Again, each chemical standard's headspace was analyzed at the end of each of their corresponding runs. By having a positive standard that can be identified at a large intensity, 
the software is capable of locating any area in the RIC that has an $\mathrm{m} / \mathrm{z}$ identical to the ion peak selected.

\subsubsection{Task 3: Part C}

The last experiment for Task 3 involves the same material as Part B. However, the methodology and exposure times differ significantly. Instead of allowing the air in the container to get saturated, the exposure of the chemical standard would begin as soon as the sample is placed onto the petri dish and the lid closed, similar to how it was done originally in Part A. Each container will have a certain number of substrates depending on the exposure times determined for that container. A table describing when the exposure times began, which containers were used, and when each container was tested is shown in Table 10.

Every substrate at each exposure time was tested in triplicate, so at any given exposure time, there are 3 toothpicks, 3 glass capillary tubes and 3 metal paper clips. There could be up to nine samples for each substrate ( 27 total) within a container if there are three exposure times being measured. For example, Box 1A indicates exposure time for the first group (1) and the letter corresponds to the chemical standard used (A: methyl benzoate, B: benzaldehyde, C: 2-ethyl-1-hexanol, D: diethyl phthalate). In total, 20 containers were used and 108 toothpicks, 108 glass capillary tubes (broken), and 108 metal clips were used.

Following each set for a chemical standard (i.e. after sampling the 9 substrate samples for methyl benzoate at 6 hour exposure time), the headspace of the standard was introduced to the DART stream to serve as a positive control. 
Table 10 - This table describes how the experiment for Task 3, Part $\mathrm{C}$ was planned. $(\mathrm{nd})=$ next day.

\begin{tabular}{|c|c|c|c|}
\hline $\begin{array}{c}\text { Time } \\
\text { Exposed }\end{array}$ & $\begin{array}{c}\text { Total Exposure } \\
\text { Times }\end{array}$ & Box \# & Time Tested \\
\hline 1:00 p.m. & 2 Hours & $1 \mathrm{~A}, 1 \mathrm{~B}, 1 \mathrm{C}, 1 \mathrm{D}$ & 3:00 p.m. \\
\hline 1:00 p.m. & 4 Hours & $1 \mathrm{~A}, 1 \mathrm{~B}, 1 \mathrm{C}, 1 \mathrm{D}$ & 5:00 p.m. \\
\hline 1:00 p.m. & 10 Hours & $1 \mathrm{~A}, 1 \mathrm{~B}, 1 \mathrm{C}, 1 \mathrm{D}$ & 11:00 p.m. \\
\hline 2:00 p.m. & 8 Hours & $2 \mathrm{~A}, 2 \mathrm{~B}, 2 \mathrm{C}, 2 \mathrm{D}$ & 10:00 p.m. \\
\hline 3:30 p.m. & 6 Hours & $2 \mathrm{~A}, 2 \mathrm{~B}, 2 \mathrm{C}, 2 \mathrm{D}$ & 9:30 p.m. \\
\hline 4:00 p.m. & 20 Hours & $3 \mathrm{~A}, 3 \mathrm{~B}, 3 \mathrm{C}, 3 \mathrm{D}$ & 12:00 p.m. (nd) \\
\hline 4:00 p.m. & 22 Hours & $3 \mathrm{~A}, 3 \mathrm{~B}, 3 \mathrm{C}, 3 \mathrm{D}$ & 2:00 p.m. (nd) \\
\hline 4:00 p.m. & 24 Hours & $3 \mathrm{~A}, 3 \mathrm{~B}, 3 \mathrm{C}, 3 \mathrm{D}$ & 4:00 p.m. (nd) \\
\hline 10:30 p.m. & 14 Hours & $4 \mathrm{~A}, 4 \mathrm{~B}, 4 \mathrm{C}, 4 \mathrm{D}$ & 12:30 p.m. (nd) \\
\hline 10:30 p.m. & 18 Hours & $4 \mathrm{~A}, 4 \mathrm{~B}, 4 \mathrm{C}, 4 \mathrm{D}$ & 4:30 p.m. (nd) \\
\hline 11:30 p.m. & 12 Hours & $5 \mathrm{~A}, 5 \mathrm{~B}, 5 \mathrm{C}, 5 \mathrm{D}$ & $11: 30$ a.m. (nd) \\
\hline 11:30 p.m. & 16 Hours & $5 \mathrm{~A}, 5 \mathrm{~B}, 5 \mathrm{C}, 5 \mathrm{D}$ & 3:30 p.m. (nd) \\
\hline
\end{tabular}

\subsubsection{Task 4: Testing COMPS' Potential for Cross-Contamination}

The fourth task involves the testing of Controlled Odor Mimic Permeation Systems (COMPS) for the possibility of cross-contamination between the training aids. The kit was made using the standard operating procedure (SOP) set forth by IFRI. Narcotic mimics differ in their method and containment to explosive mimics. The methodology for making these kits is as follows:

\section{Explosive training aid kit}

The work area was cleared, cleaned and dried. Butcher paper was laid on the work area, and replaced between each time a different mimic was made. Proper protective equipment (PPE) of gloves, dust mask and goggles were worn, and the gloves were replaced between each mimic was made as well. The LDPE bags were then prepared and 2 mil LDPE bags to be used for each explosive training aid mimic. Below the initial heat seal, each bag was labeled with the type of training aid, lot \# and number of mimic. Next, 
the bags are placed into their corresponding jar and prior to each preparation, two additional heat seals slightly above the original heat seal were added.

The blanks were prepared first. The first blank involves no material, whereas the second black is made up of two 2" x 2" 8 ply gauze pads in each LDPE bag. For the remaining mimics, the analytical balance was used to weigh the material on a weight boat (except for the liquid 2-ethyl-1-hexanol, which was weighed in a $100 \mathrm{~mL}$ beaker). The TNT mimic was made using $25 \mathrm{~g}$ of Hodgson $\mathrm{H} 4895$ Smokeless Powder in each LDPE bag. The nitroglycerin mimic was made using $25 \mathrm{~g}$ of Winchester 760 Smokeless Powder in each bag. The tagged mimic was made using $3 \mathrm{~g}$ of 2,3-dimethyl-2,3-dintrobutane in each bag. The smokeless powder \#1 mimic was made using 3g of 1,3-diethyl-1,3-diphenylurea in each bag. The smokeless powder \#2 mimic was made using $3 \mathrm{~g}$ of diphenylamine in each bag. Finally, the plasticized mimic was made using 2-ethyl-1-hexanol.

After each bag is filled, three heat seals are added at the top of the bags (no more than $3 \mathrm{~cm}$ from the top). Once sealed, the bags are added back into the mason jars and securely closed.

\section{Drug training aid kit}

Similarly, the work area is first cleared, cleaned and dried prior to starting and sheets of butcher paper were then placed on the work area. Gloves, dust masks and goggles (PPE) were worn and gloves were replaced between the making of each mimic.

Blank mimics were then made for cocaine, methamphetamine and MDMA. For both cocaine and methamphetamine blanks, 30g of cellulose ( 20 micron powder) were weighed using a top loading balance on a 4" weigh boat. For the MDMA blank, 30 grams 
of alumina powder (80-200 mesh) were weighed out. The powder is then placed into a 6" x 5.5" Barrier foil ziplock bag that was labeled prior. Next, three heat seals are added above the tear notch. This heat-sealed bag is then placed into the outer 8 " 10.5 " 13.5 " Barrier foil ziplock bag that was also labeled prior. Finally, three heat seals are added to the top of the bag above the tear notch.

For the drug mimics, the procedure is identical but the contents differ. Each training aid is mixed in a separate mixing jar prior to placing in the bag. For both the cocaine and methamphetamine mimics, $100 \mathrm{~g}$ of cellulose ( 20 micron powder) is used whereas $90 \mathrm{~g}$ of alumina powder is used for the MDMA mimic. In the cocaine mimic mixing jar, $150 \mu \mathrm{L}$ methyl benzoate is added to the cellulose. The methamphetamine training mimic uses $0.75 \mathrm{~g}$ of benzaldehyde was added to the cellulose. The MDMA training aid uses $10 \mathrm{~g}$ of piperonal were added to the $90 \mathrm{~g}$ of alumina powder.

Each mixing jar is then covered and wrapped with parafilm and shaken for five minutes every half hour for a minimum of two hours. A new weigh boat is placed onto a top loading balance and each mixture was weighed out and then added into the corresponding 6" x 5.5" Barrier foil ziplock bag. Three heat seals are then added above the tear notch, and then this sealed bag is placed into the outer, labeled 8 " $10.5 " \times 3.5$ " Barrier foil ziplock bags.

\section{Testing for Possibility of Cross-Contamination Using DART}

Prior to opening any of the training aids, the exterior of the jar (for explosive mimics) and outer Barrier foil ziplock bag (for narcotic mimics) were tested directly using the DART. The sample gap between the DART and the orifice is $10 \mathrm{~cm}$ and the mason jars 
are much wider, so they were placed right by the DART (Figure 39) and the experiment was run for 5 minutes for each sample. The extended time is to ensure that no chemical or volatile compound is slowly leaking out of the sealed jars. This was not an issue for the Barrier foil ziplock bags, as the edges were analyzed directly into the DART stream.

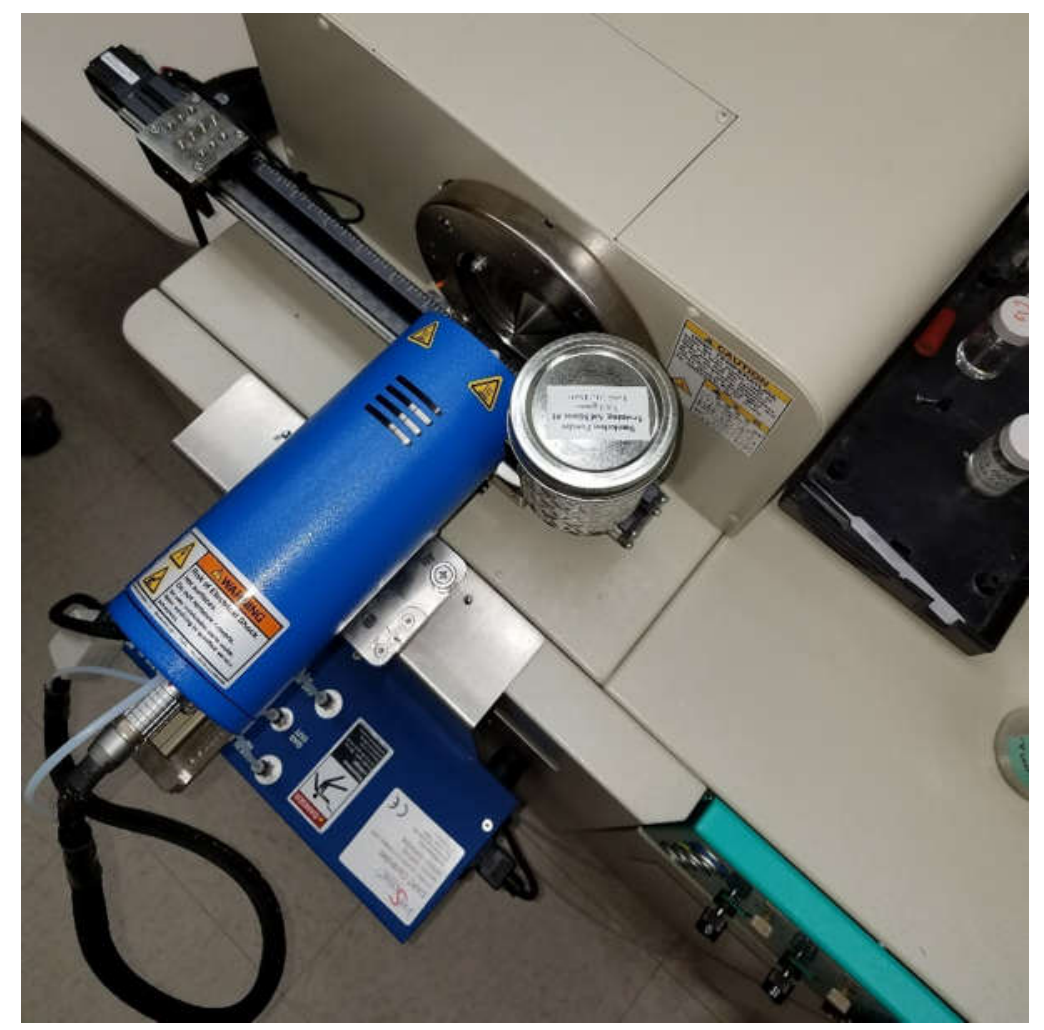

Figure 39 - Sampling the exterior of the mason jars.

Following the sampling of the exteriors of the secondary containers (outer bag and jar), they were opened and their contents were sampled directly using the DART. For the narcotic mimic, this involved the use of the primary, smaller, Barrier foil ziplock bag (unopened) and for the explosive mimics, this involved the permeable LDPE bags containing the mimics. For the explosive mimics, the LDPE bags were sampled next to the DART stream, and care was taken to not have the bag come in direct contact with the 
DART stream, as this would result in the LDPE bag melting and its contents would in turn be directly exposed (Figure 41). This was not an issue for the smaller Barrier foil bag as it was unopened and is not permeable. The exterior of the smaller bag was then sampled directly prior to opening and after opening the bag (while ziplock sealed), as well as sampling the headspace of the contents of the small bag itself.

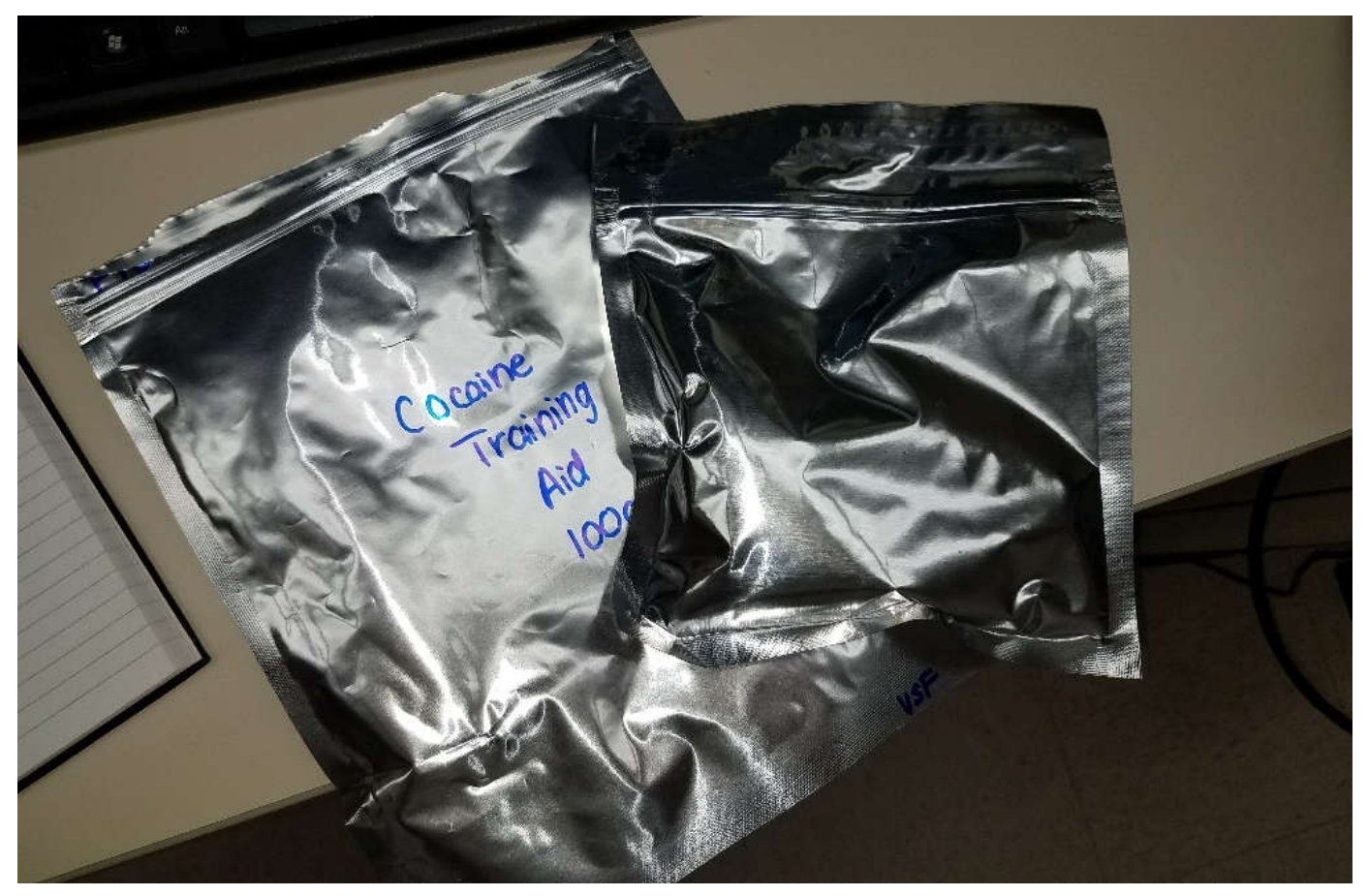

Figure 40 - Picture of the primary (right) and secondary (left) containers used for the narcotic mimics. 


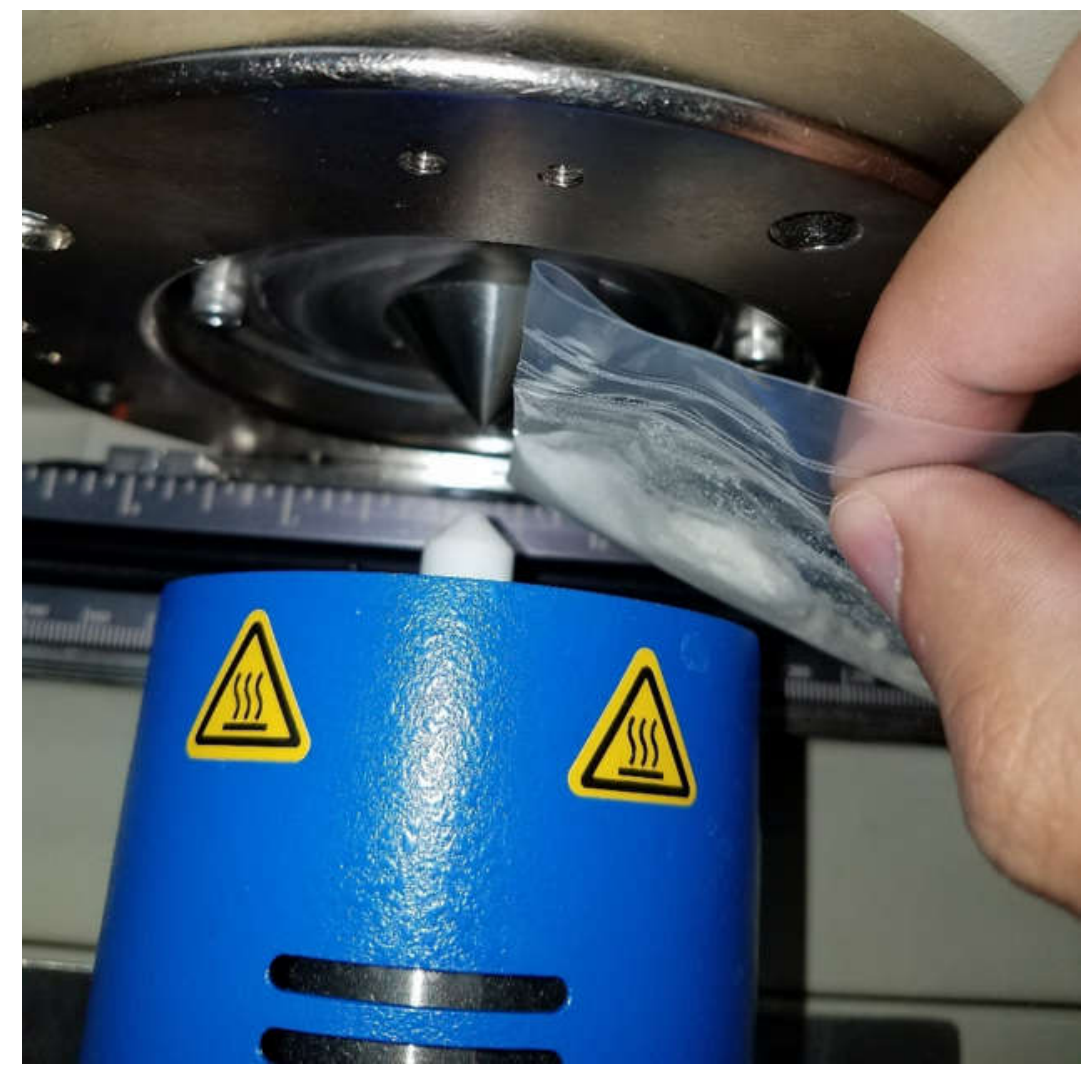

Figure 41 - Sampling of the LDPE bags. This needed to be carefully done so that the DART stream does not melt the bag and expose its contents.

\subsubsection{Task 5: Survey of Narcotic and Explosive Detecting Canine Handlers}

The fifth and final task involved the use of a survey. The questionnaire is to be answered by narcotic and explosive canine handlers at various agencies (federal, state, county, etc.). The survey was created using Google Forms (https://docs.google.com/forms/u/0/). Some questions were multiple choice and others required a short answer. The questions and the layout are as follows: 
1. Do you work for a County, State or Federal Agency?
a. County
b. State
c. Federal
d. Other

2. Do you use commercially available training aids, in-house prepared pseudo scent training aids, or actual drugs/explosives to train your canine teams?
a. Commercially available training aids
b. In-house prepared pseudo scent training aids
c. Actual drug/explosive

3. In your opinion, does one type or brand of training aid work better than any other Please briefly explain your response.

a. (Short answer text)

4. Have you observed unconfirmed alerts that can be attributed to the close proximity of targets to illicit substances?
a. Yes
b. No
c. Maybe

5. Have you observed unconfirmed alerts that can be attributed to potential storage issues?
a. Yes
b. No
c. Maybe 
6. Do you believe unconfirmed alerts can be attributed to an illicit substance recently being present in that particular location?
a. Yes
b. No
c. Maybe

7. Would you be willing to participate in a scientific study testing new training aids for effectiveness?
a. Yes
b. No

8. How are the training aids contained or stored between training?
a. Glass Jars
b. Plastic Jars
c. Metal Cans
d. Plastic Bags
e. Other

9. What is the shelf life of the training aids used to train the canines?
a. (Short answer text)

10. How often are your training aids changed to new aids?
a. Monthly
b. Seasonally
c. Every 6 months
d. Yearly
e. Other 
11. What alert percentage do you think is appropriate to certify a canine for the detection of illicit substances?
a. $100 \%$
b. Above $90 \%$
c. Above $75 \%$
d. Above 50\%
e. Other

12. How often do you train the canines?
a. Daily
b. Weekly
c. Monthly
d. Other

13. Are you familiar with SWGDOG?
a. Yes
b. No

14. Are you familiar with OSAC?
a. Yes
b. No

15. Are your canine teams certified and under what standard?

a. (Short answer text)

16. Are there any suggestions you would like SWGDOG or OSAC to consider?

a. (Long answer text) 


\section{RESULTS:}

\subsection{Task 1: Optimizing DART Parameters}

When it comes to the various parameters than can be adjusted with the DART and mass spectrometer (as described in the materials and methods section), there are two major parameters that are adjusted by the user based on the sample type: (1) DART gas stream temperature and (2) orifice 1 voltage.

With respect to the orifice voltage, the range recommended by the manufacturer is $20-90$ volts. The higher the voltage, the higher the fragmentation. This research did not require the use of more fragmentation due to the fact that known standards were used, and the creation of more fragment ions in the spectra can make for a messier result. Therefore, the choice was between 20 and 30 volts.

During function switching, 20 volts serves as the function with little-to-none fragmentation. At 30 volts, the user will get a little more fragmentation, and is often the voltage of choice when a single voltage is used instead of function switching. For these reasons, an orifice voltage of 30 volts was chosen for the rest of this research.

As for temperature, each sample has a different boiling point and ionization energy from the other. Therefore, the goal is to find a temperature suitable for all samples, in which they can be identified when analyzed via headspace. To do this, each sample was tested at temperatures ranging from $150^{\circ} \mathrm{C}-500^{\circ} \mathrm{C}$ at $50^{\circ} \mathrm{C}$ intervals.

To ensure that the calibrant, PEG 600, is tested properly, each run was started at $300^{\circ} \mathrm{C}$ or $350^{\circ}$, as it is more difficult to see higher PEG 600 mass peaks at lower temperatures and is inversely problematic at higher temperatures. 
Each of the four standards were analyzed separately, with the first run ranging in temperature from $300^{\circ} \mathrm{C}$ to $150^{\circ} \mathrm{C}$. At each interval, each standard was tested in triplicate (Figure 42-Figure 49.

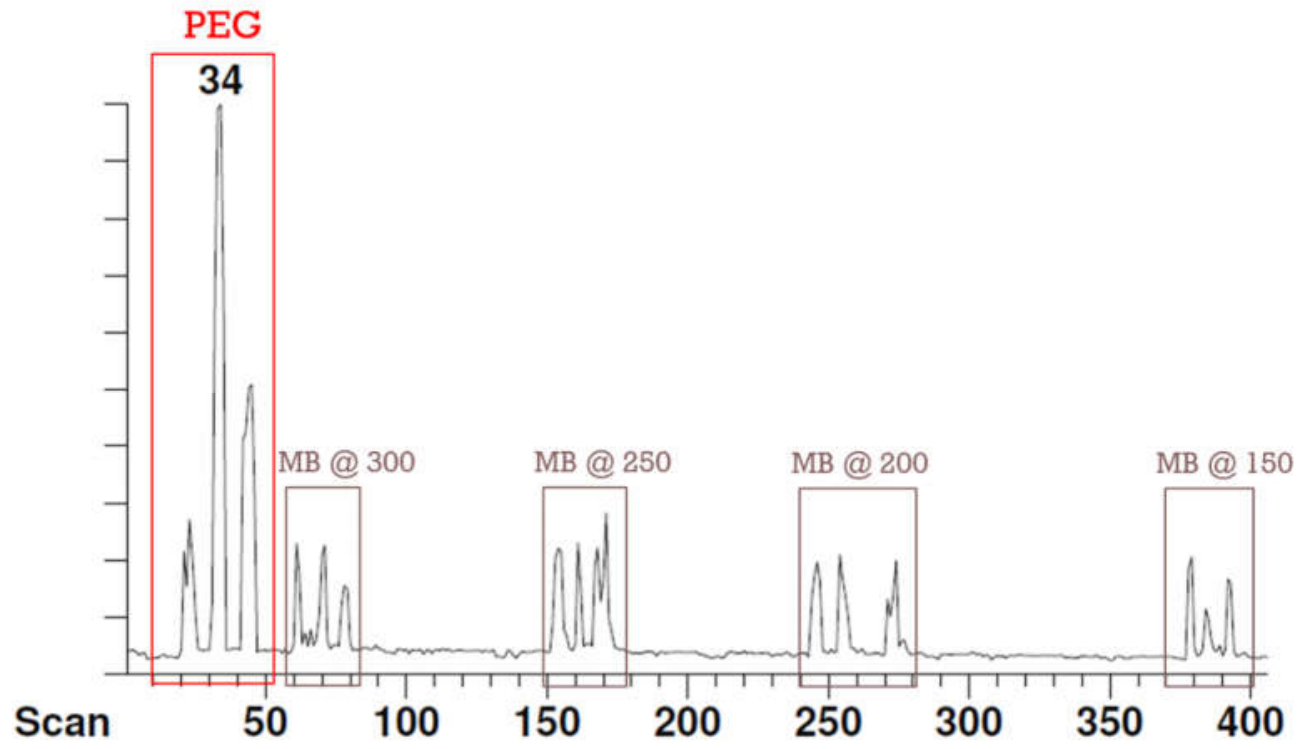

Figure 42 - RIC of methyl benzoate $300^{\circ} \mathrm{C}-150^{\circ} \mathrm{C}$.

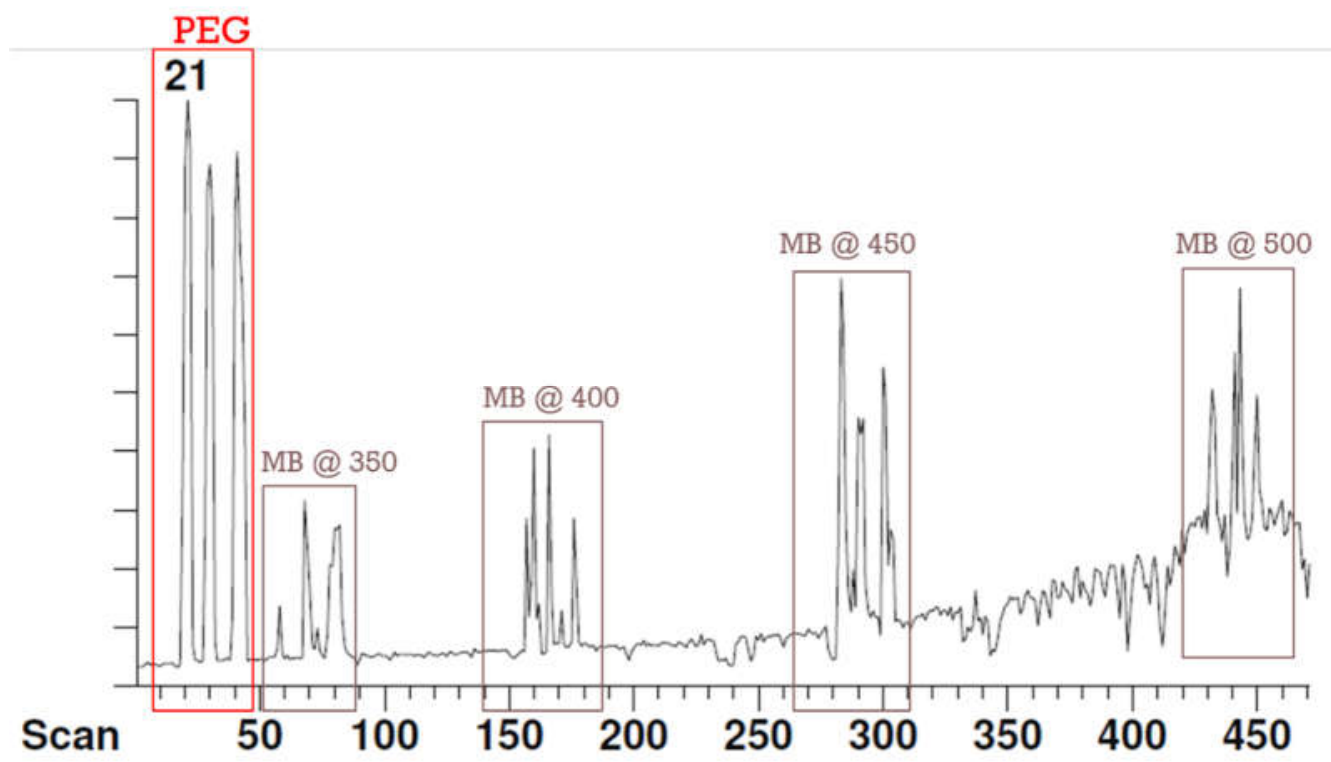


Figure 43 - RIC of methyl benzoate $350^{\circ} \mathrm{C}-500^{\circ} \mathrm{C}$

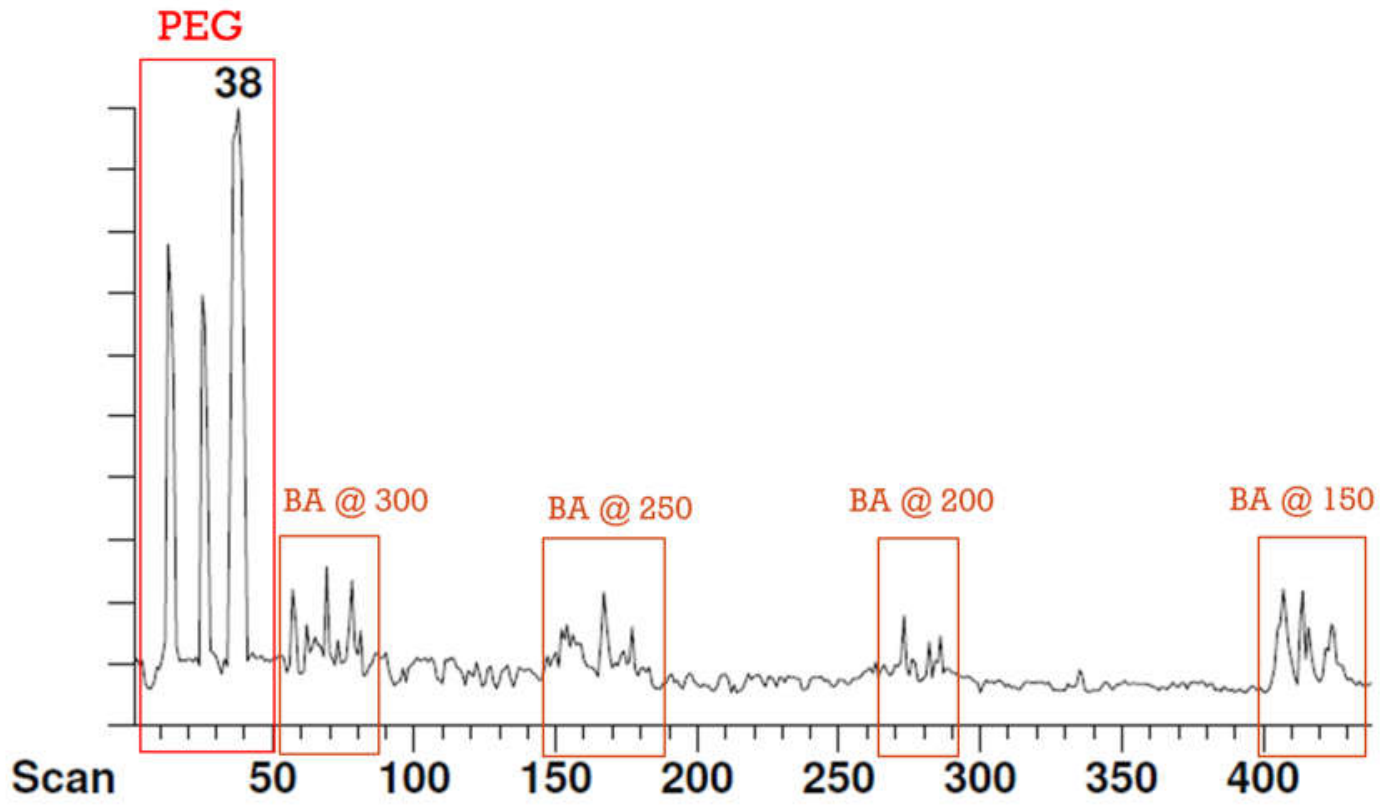

Figure 44 - RIC of benzaldehyde $300^{\circ} \mathrm{C}-150^{\circ} \mathrm{C}$

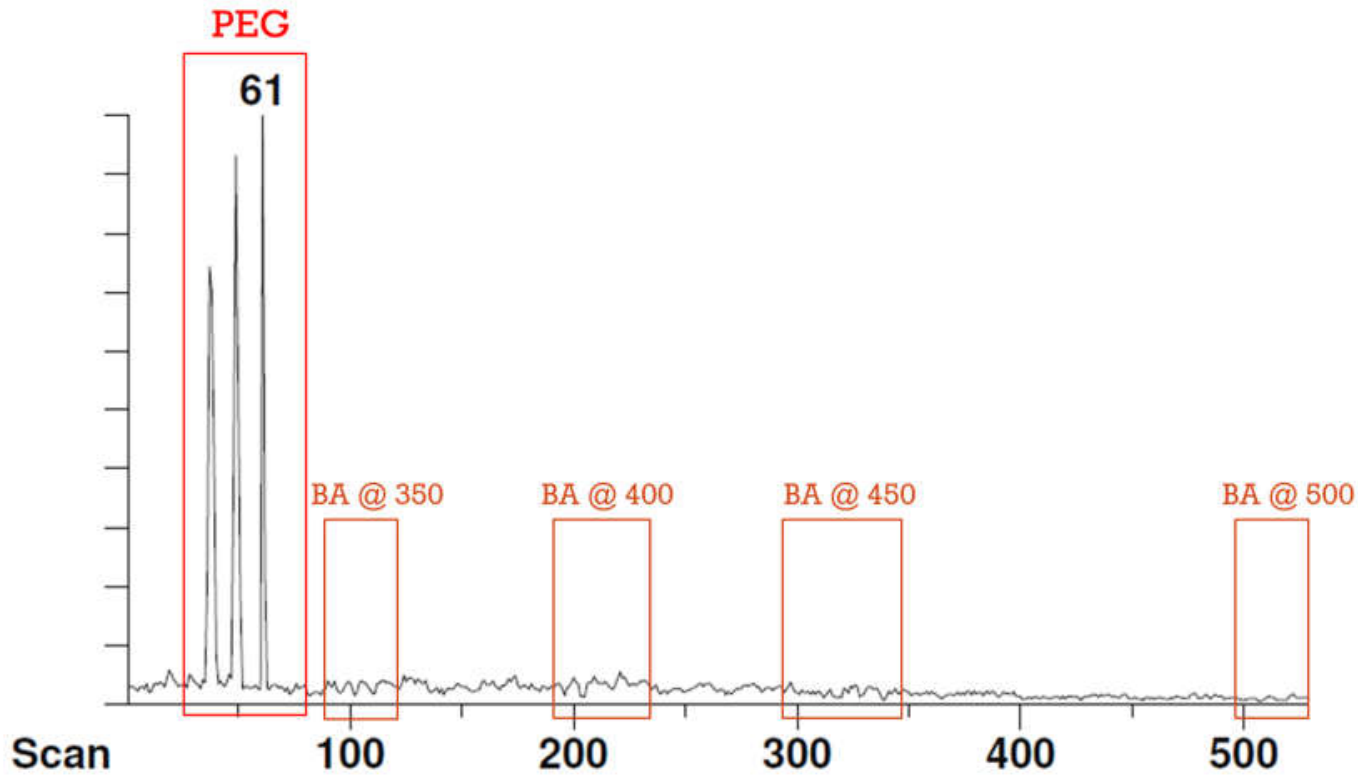

Figure $45-$ RIC of benzaldehyde $350^{\circ} \mathrm{C}-500^{\circ} \mathrm{C}$. 


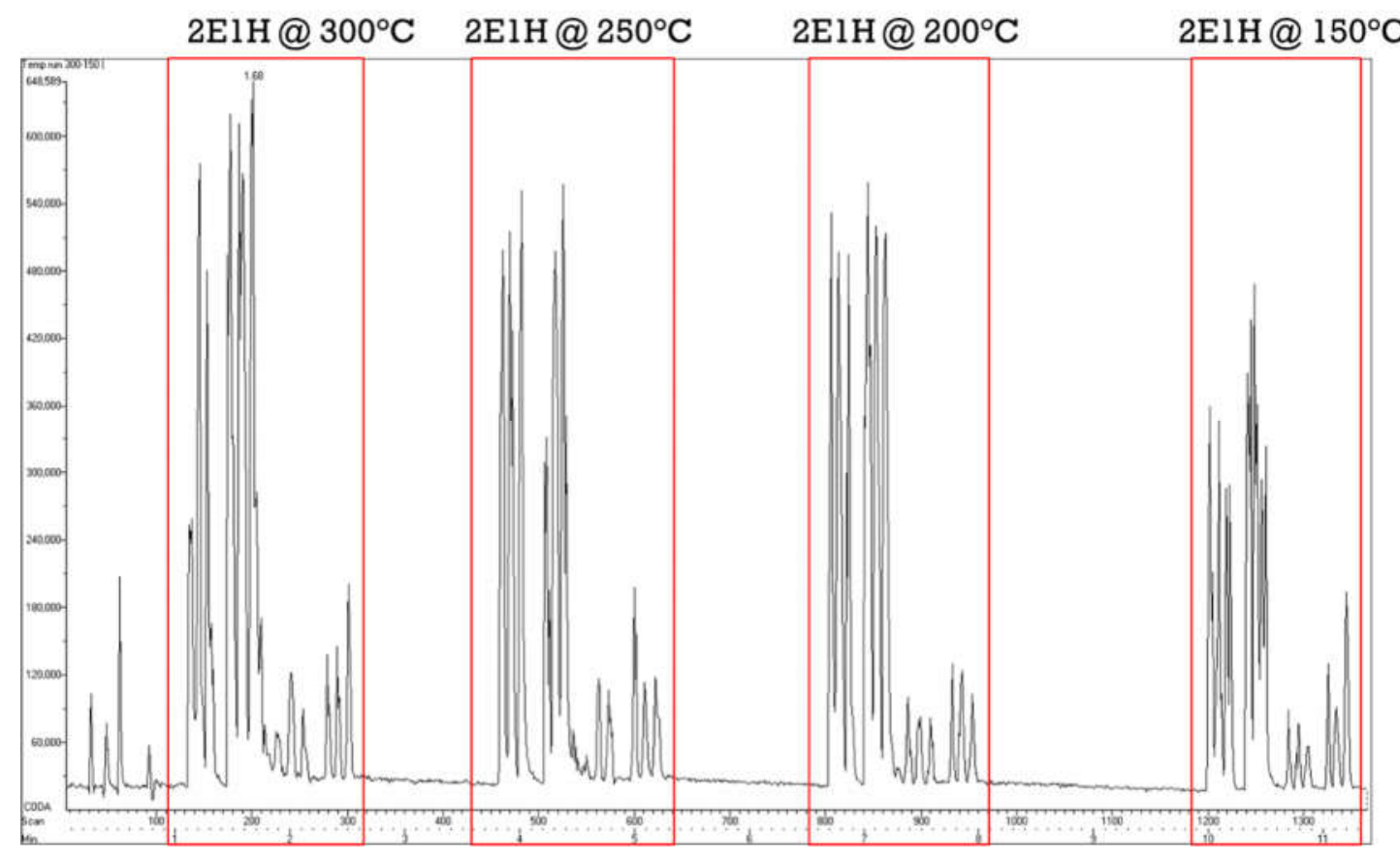

Figure 46 - RIC of 2-ethyl-1-hexanol $300^{\circ} \mathrm{C}-150^{\circ} \mathrm{C}$.

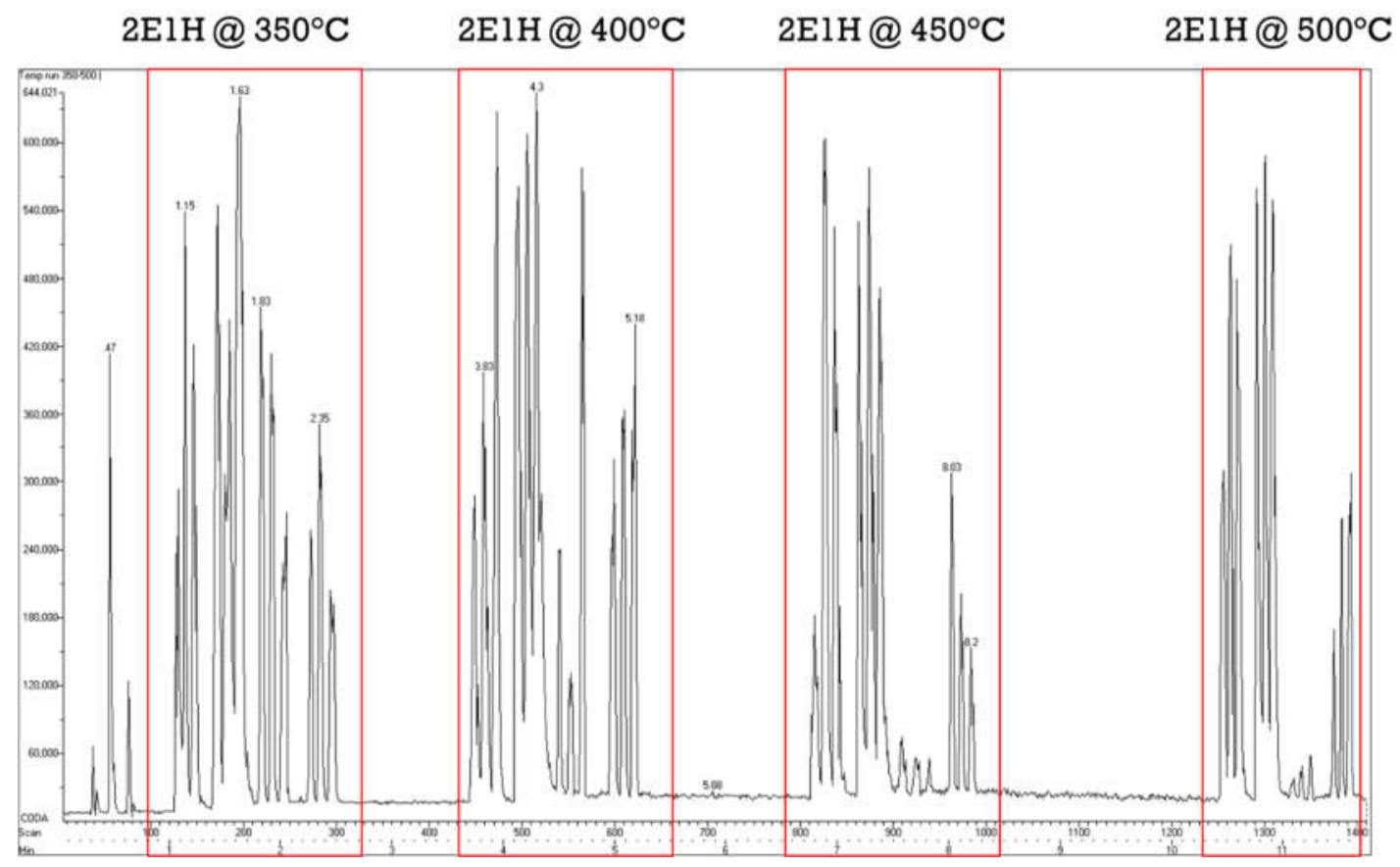

Figure 47 - RIC of 2-ethyl-1-hexanol $350^{\circ} \mathrm{C}-500^{\circ} \mathrm{C}$. 


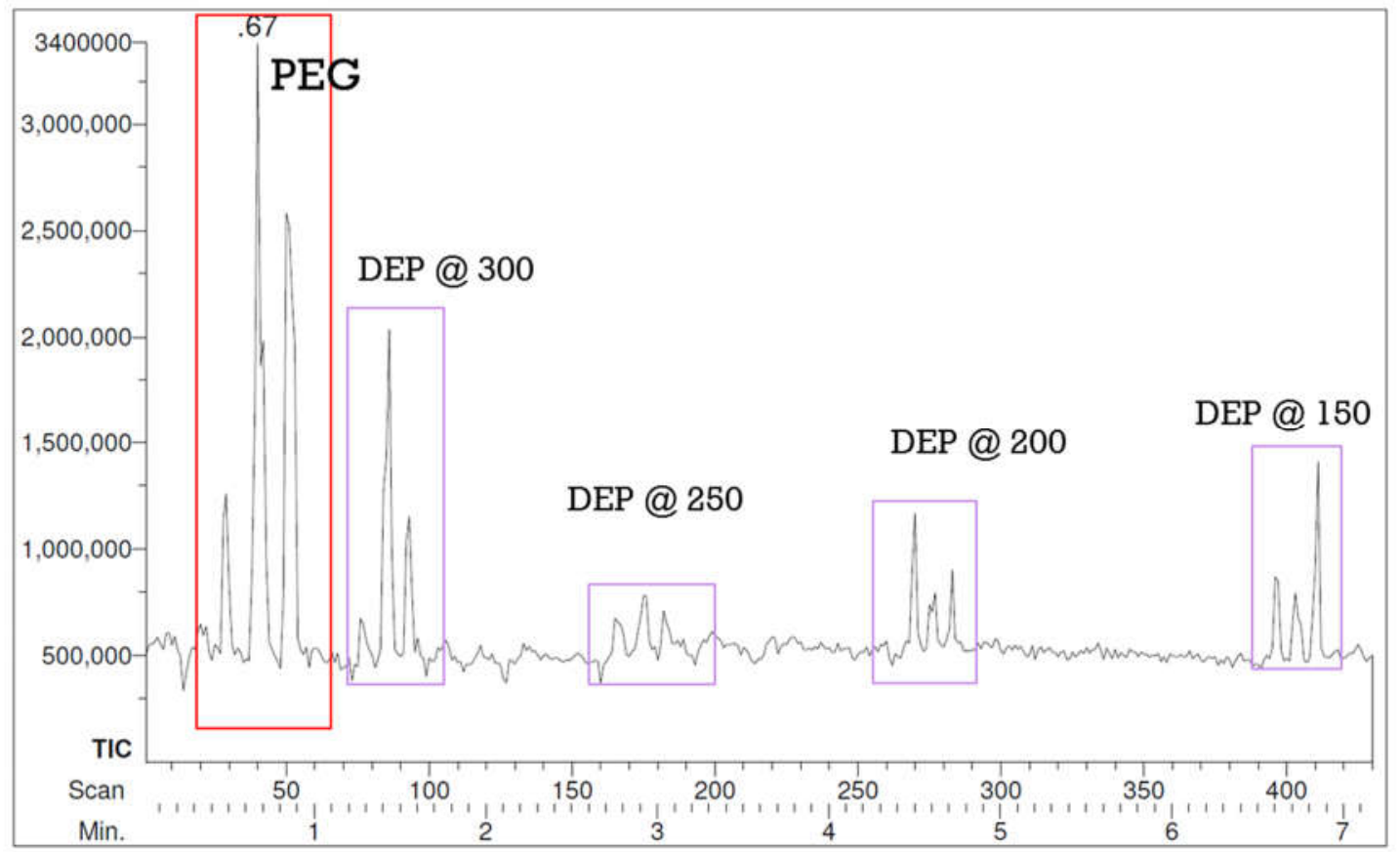

Figure 48 - RIC of diethyl phthalate $300^{\circ} \mathrm{C}-150^{\circ} \mathrm{C}$.

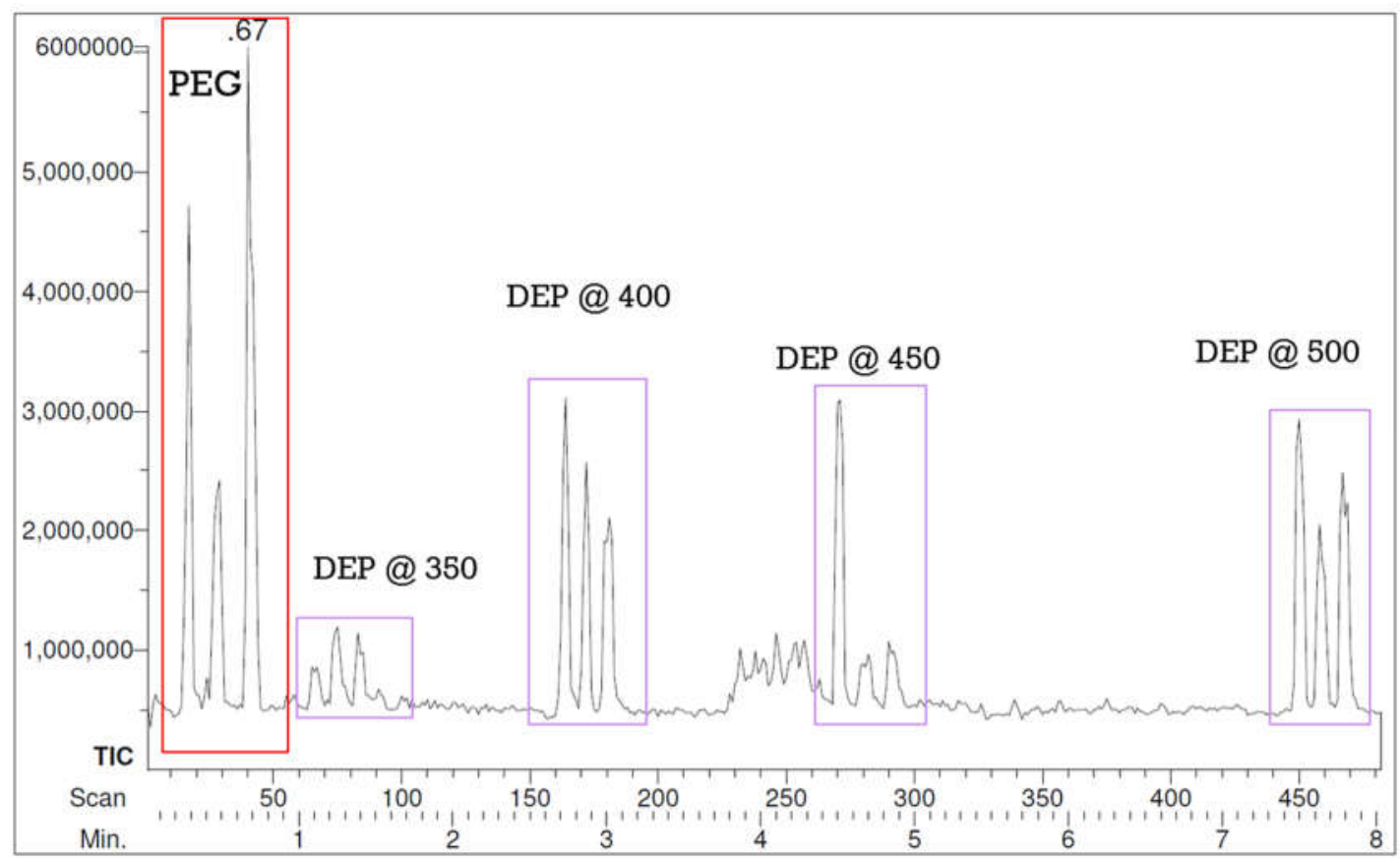

Figure $49-$ RIC of diethyl phthalate $350^{\circ} \mathrm{C}-500^{\circ} \mathrm{C}$. 
The figures above show RIC's for one of the three runs, as each test was done in triplicate. Within each run, every sample was tested in triplicate as well, which can be identified by the three peaks in each temperature range. Each peak in the RIC was 'averaged' to create a mass spectrum. Examples for the mass spectra at each temperature for methyl benzoate and benzaldehyde are shown in Figure 50-Figure 53. Again, only one mass spectrum of the three collected is shown in those figures. Of those three collected, the median intensity was chosen and used to determine the optimal temperature. 


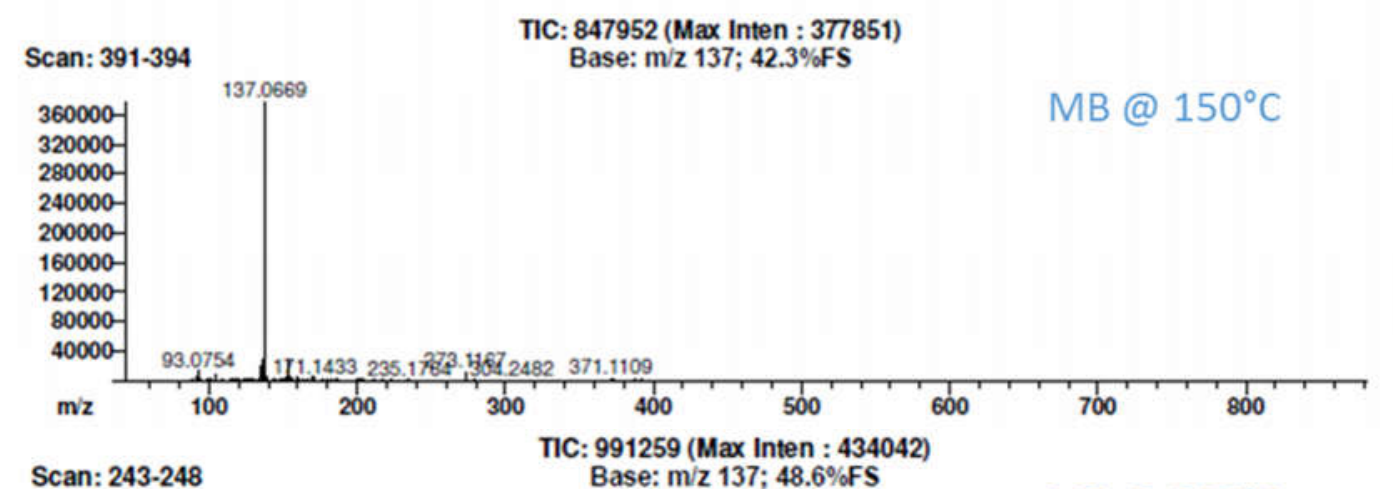

Scan: $243-248$

Base: $\mathrm{m} / \mathrm{z} 137 ; 48.6 \% \mathrm{FS}$
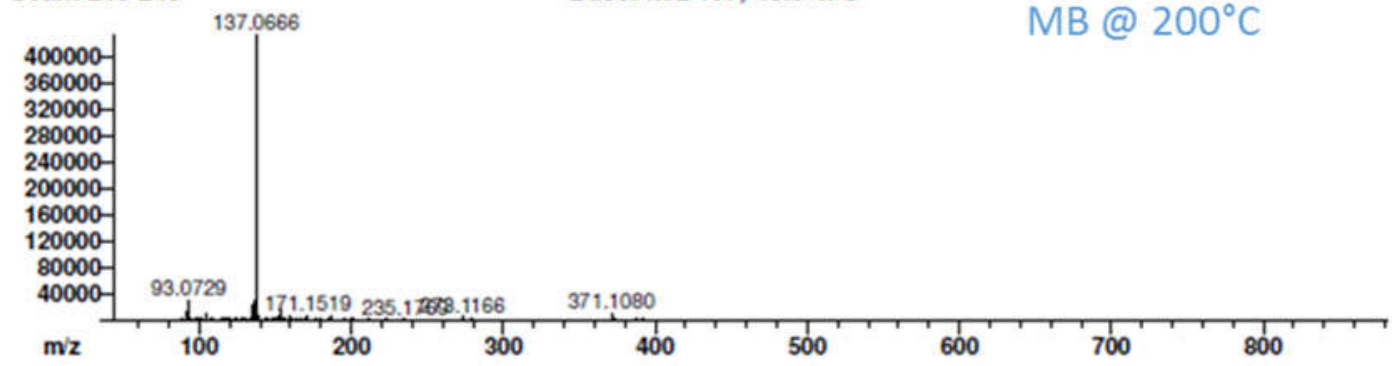

m/z

TIC: 1132817 (Max Inten : 509562)

Scan: $166-174$

Base: $\mathrm{m} / \mathrm{z} 137 ; 57.1 \% \mathrm{FS}$

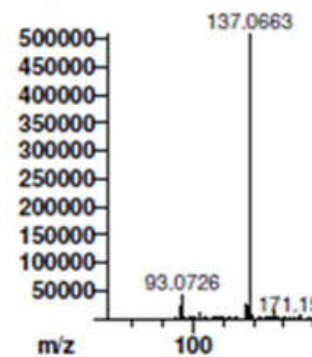

$\mathrm{m} / \mathbf{z}$

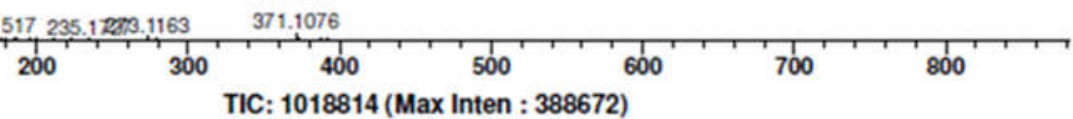

Scan: $68-72$

Base: $\mathrm{m} / \mathrm{z} 137 ; 43.6 \% \mathrm{FS}$

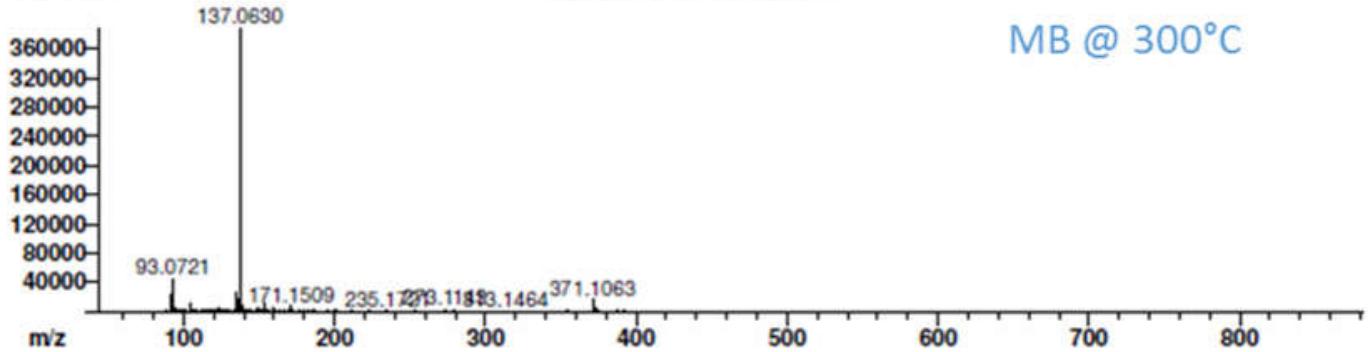

Figure 50 - Mass spectra of methyl benzoate at various temperatures $\left(150^{\circ} \mathrm{C}-300^{\circ}\right)$. 


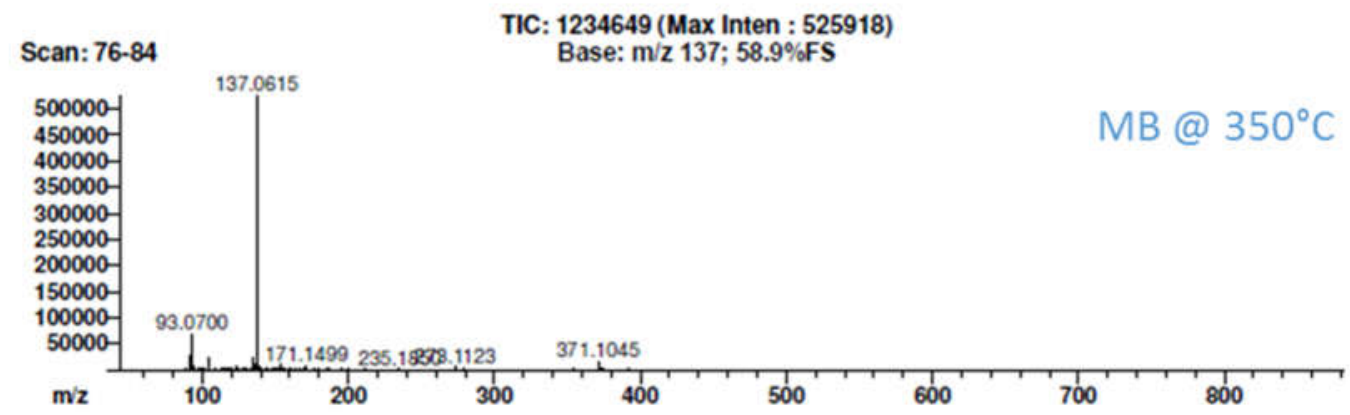

TIC: 1235701 (Max Inten : 428616)

Scan: 156-163

Base: $\mathrm{m} / \mathrm{z} 137 ; 48 \% \mathrm{FS}$

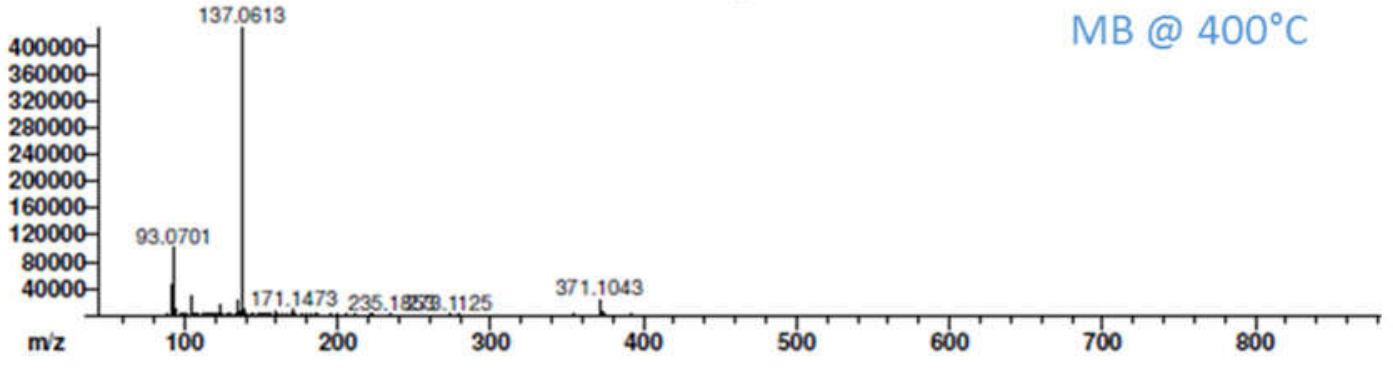

TIC: 2158914 (Max Inten : 611582)

Scan: $289-293$

Base: $\mathrm{m} / \mathrm{z} 137 ; 68.5 \% \mathrm{FS}$

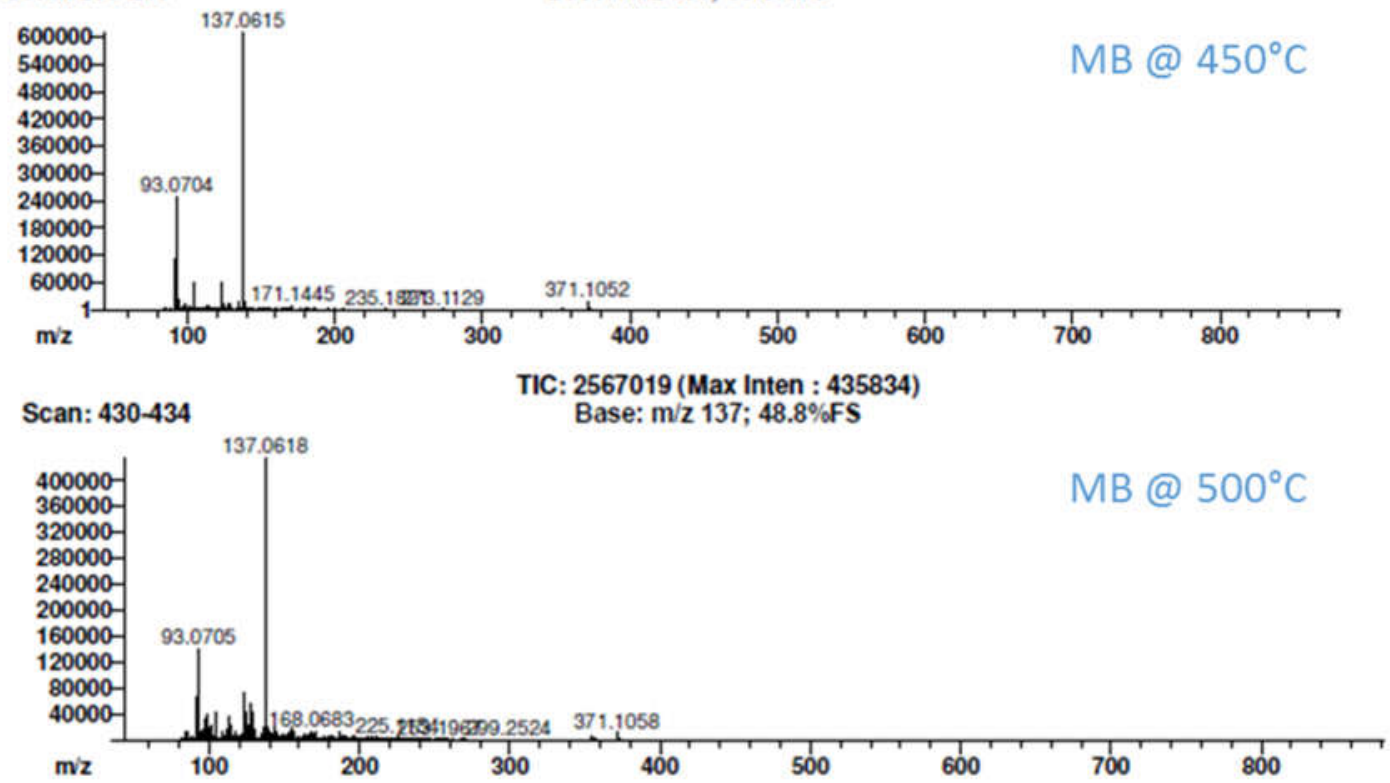

Figure 51 - Mass spectra of methyl benzoate at various temperatures $\left(350^{\circ} \mathrm{C}-500^{\circ}\right)$. 

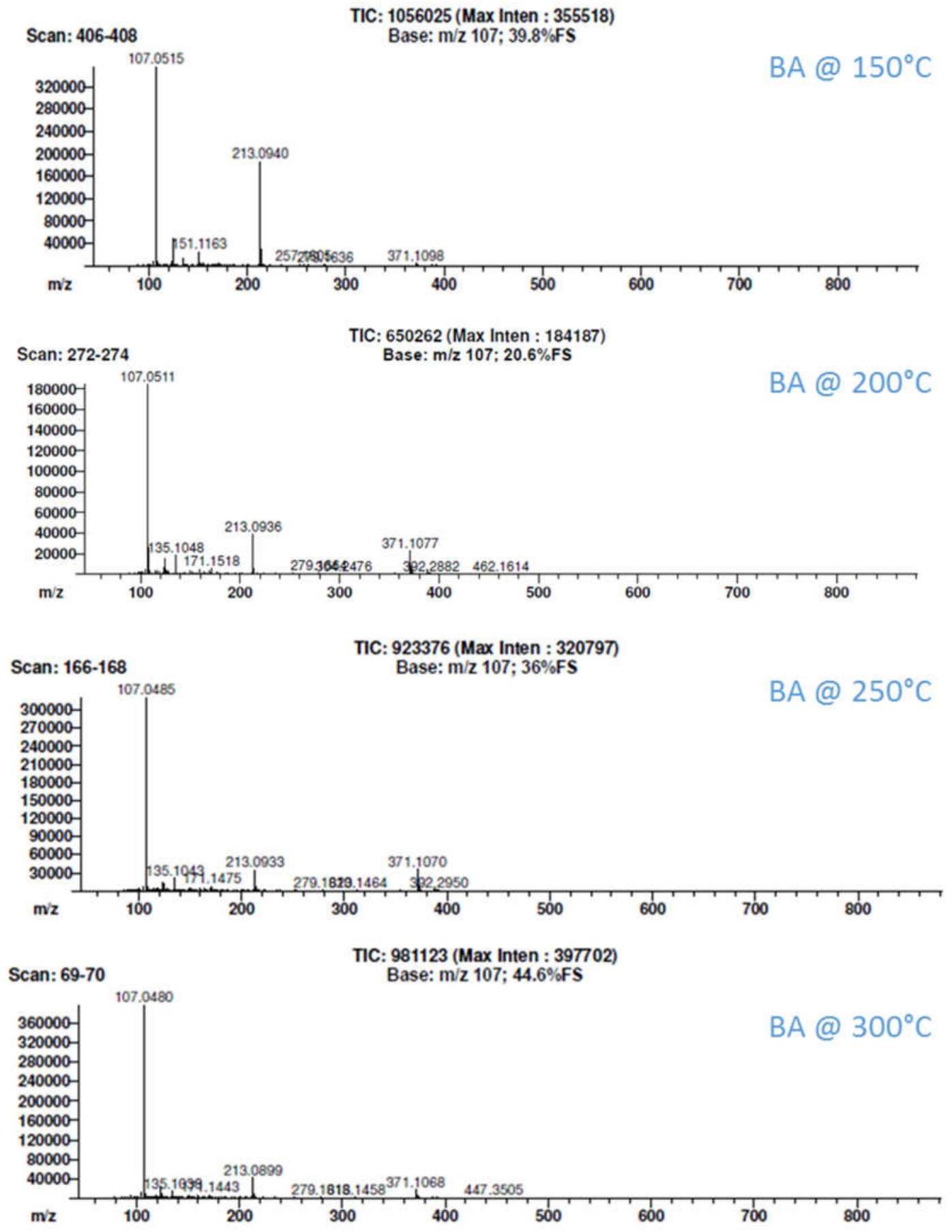

Figure 52 - Mass spectra of benzaldehyde at various temperatures $\left(150^{\circ} \mathrm{C}-300^{\circ}\right)$. 

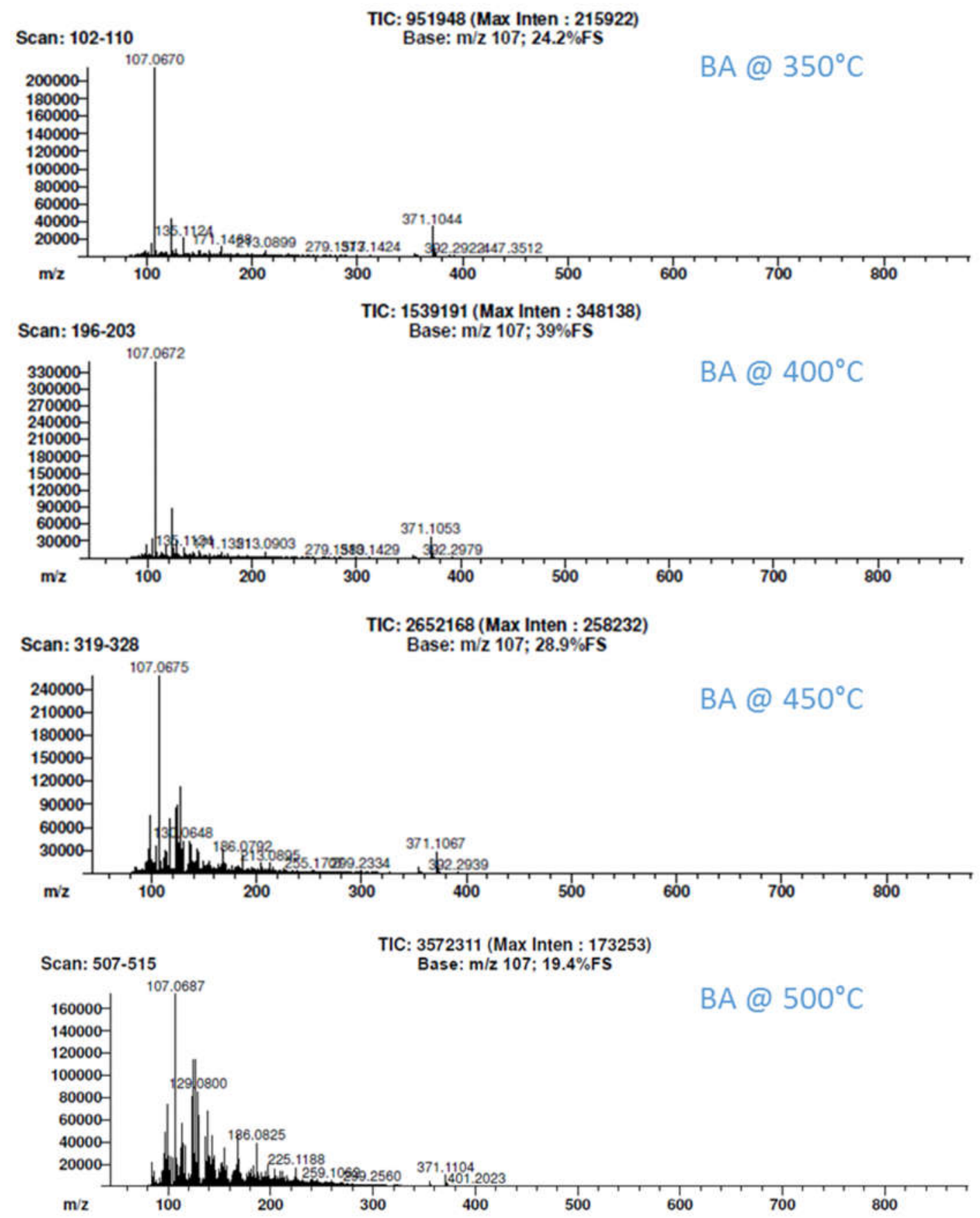

Figure 53 - Mass spectra of benzaldehyde at the various temperatures $\left(350^{\circ} \mathrm{C}-500^{\circ}\right)$.

When looking at Figure 50Figure 53 at the higher temperature ranges, the spectra contains more ions simply due to pyrolysis. It can also be seen that the parent ion is indeed the molecular ion. This is not the case with 2-ethyl-1-hexanol, which was initially 
problematic, since the molecular ion peak $([\mathrm{M}+\mathrm{H}]=131.238 \mathrm{~m} / \mathrm{z})$ could not be seen or identified at any of the temperatures (Figure 54).

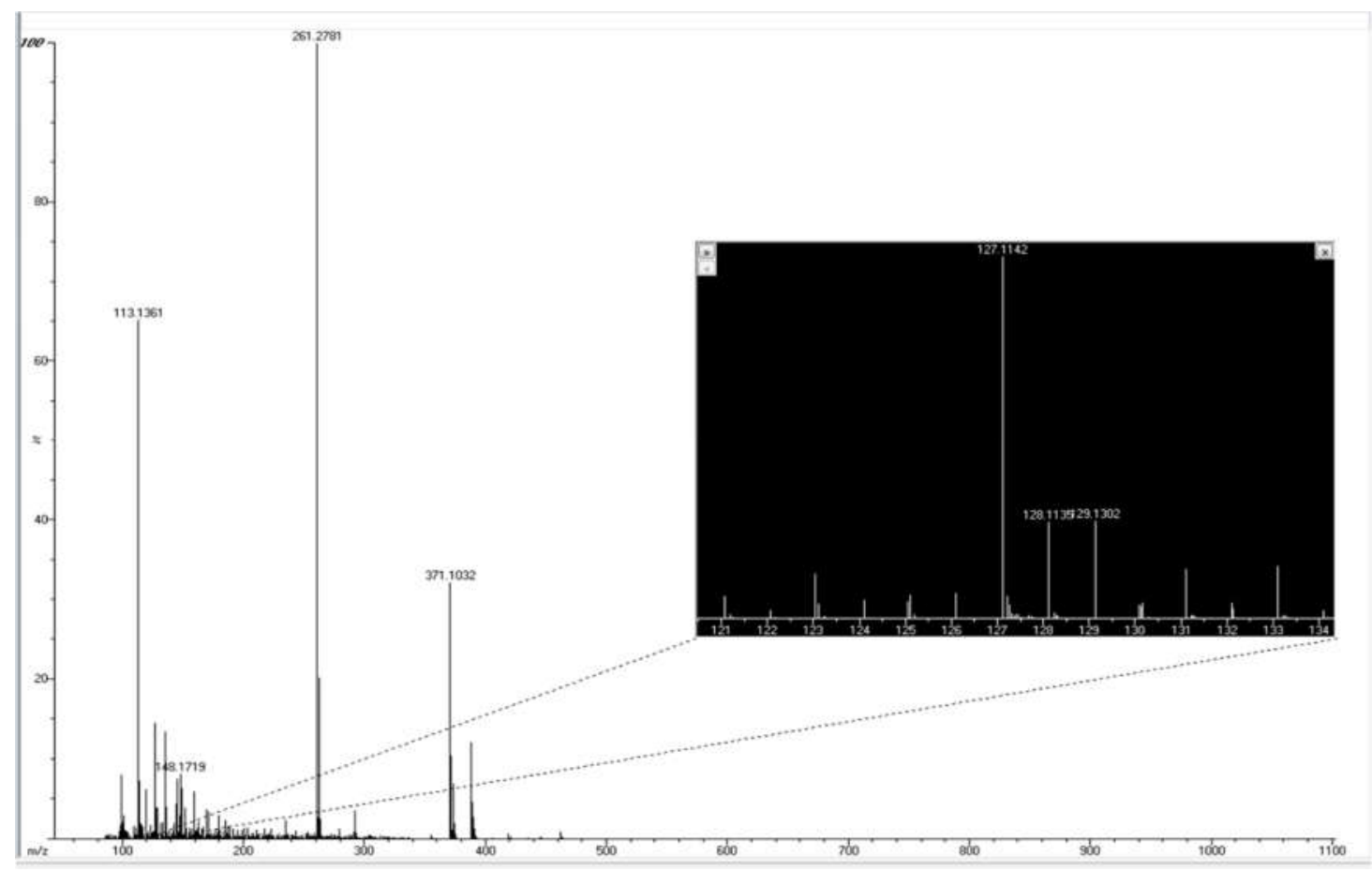

Figure 54 - Mass spectrum of 2-ethyl-1-hexanol at $150^{\circ} \mathrm{C}(30 \mathrm{~V})$.

Instead, the protonated dimer $([2 \mathrm{M}+\mathrm{H}]=261.461 \mathrm{~m} / \mathrm{z})$ was consistently observed. The next step was to determine whether a higher orifice voltage could produce $\mathrm{M}+\mathrm{H}$ via collision induced dissociation. That, too, was not successful. In fact, the $2 \mathrm{M}+\mathrm{H}$ dimer was not observed at all (Figure 56). The black box in the mass spectrum is magnifying an approximate $50 \mathrm{~m} / \mathrm{z}$ range to see if $\mathrm{M}+\mathrm{H}$ could be observed.

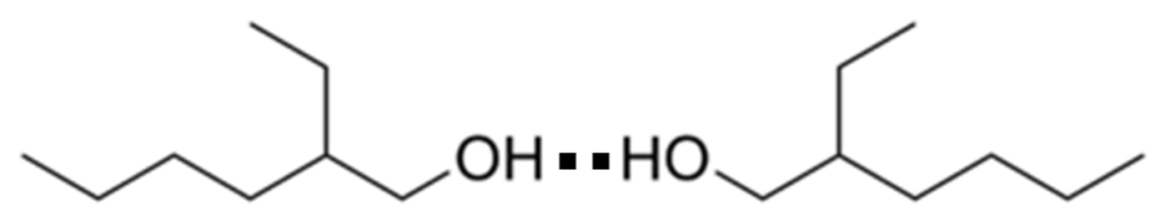

Figure 55 - 2-ethyl-1-hexanol dimer. 


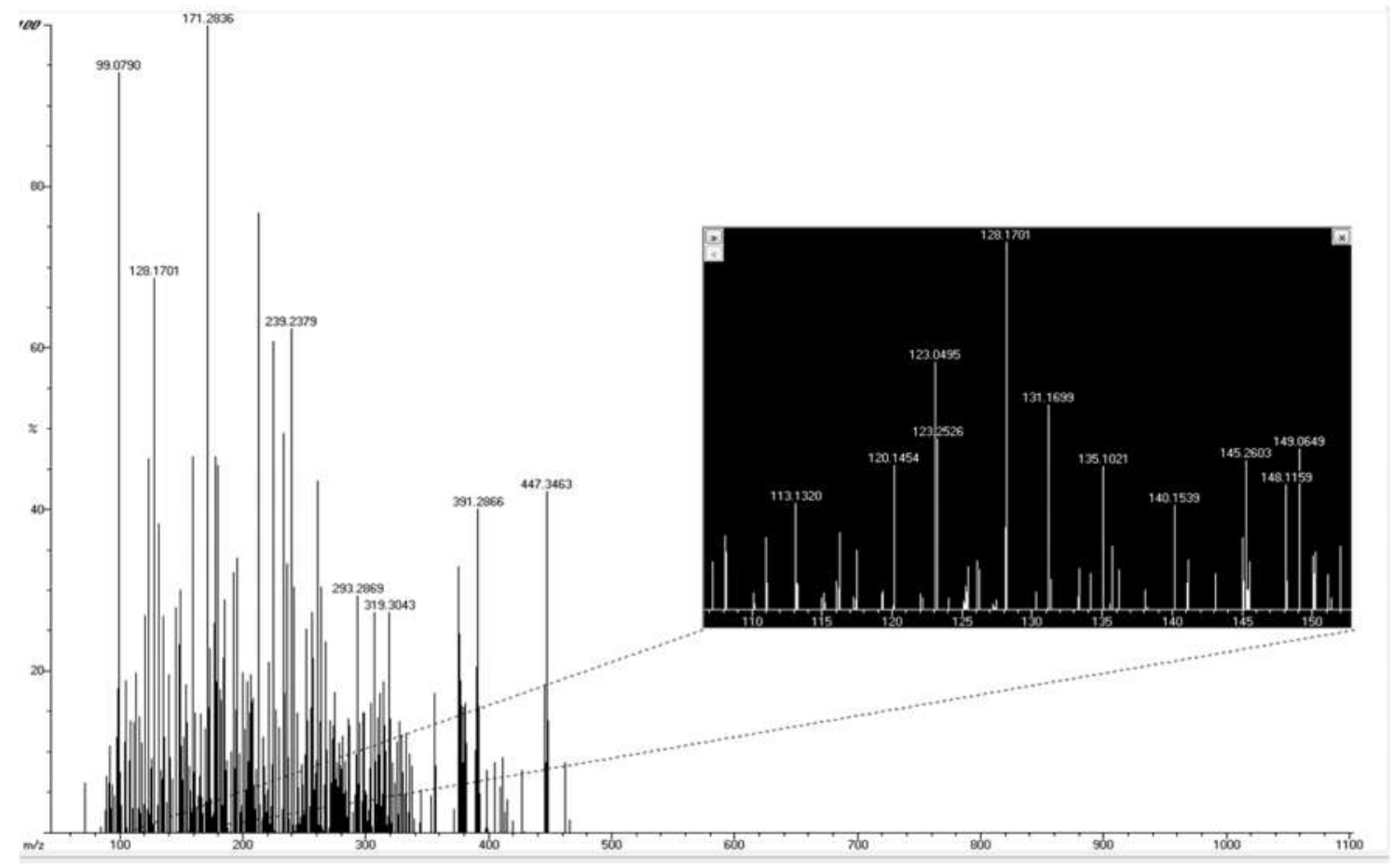

Figure 56 - Mass spectrum of 2-ethyl-1-hexanol at $350^{\circ} \mathrm{C}(90 \mathrm{~V})$.

Despite there being a peak at $131.169 \mathrm{~m} / \mathrm{z}$, it would not be accurate to label as 2ethyl-1-hexanol for two reasons: (1) it does not fall within $\pm 5 \mathrm{mDa}$ of the calculated value for $\mathrm{M}+\mathrm{H}$ and (2) it is well below the $5 \%$ threshold for peak intensity. Since the dimer $2 \mathrm{M}+\mathrm{H}$ was identified throughout these experiments, this peak was chosen to identify 2ethyl-1-hexanol for the remainder of this study. 

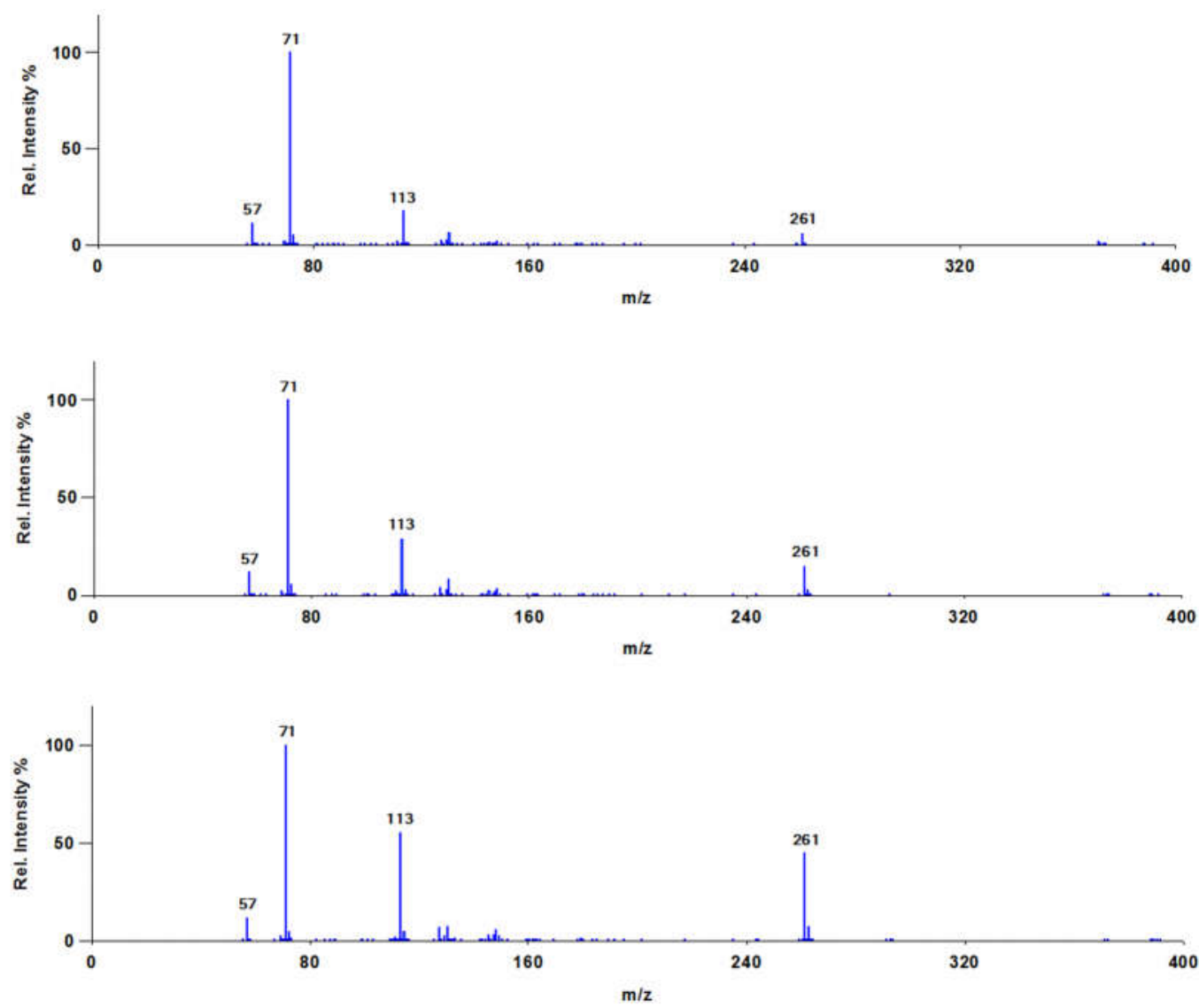

Figure 57 - Mass spectra of 2-ethyl-1-hexanol at temperatures of $200^{\circ} \mathrm{C}$ (top), $250^{\circ} \mathrm{C}$ (middle) and $300^{\circ} \mathrm{C}$ (bottom).

As stated prior, 2,4-ditrotoluene (2,4-DNT) was originally chosen as a sample in this study due to its prevalence as a primary odorant in TNT and cast explosives (Table 6). However, 2,4-DNT can only be analyzed in negative ion mode (Table 7). When attempting to analyze the sample in positive ion mode, there was no identifiable peak present that could be used for the remainder of this study (Figure 58). 


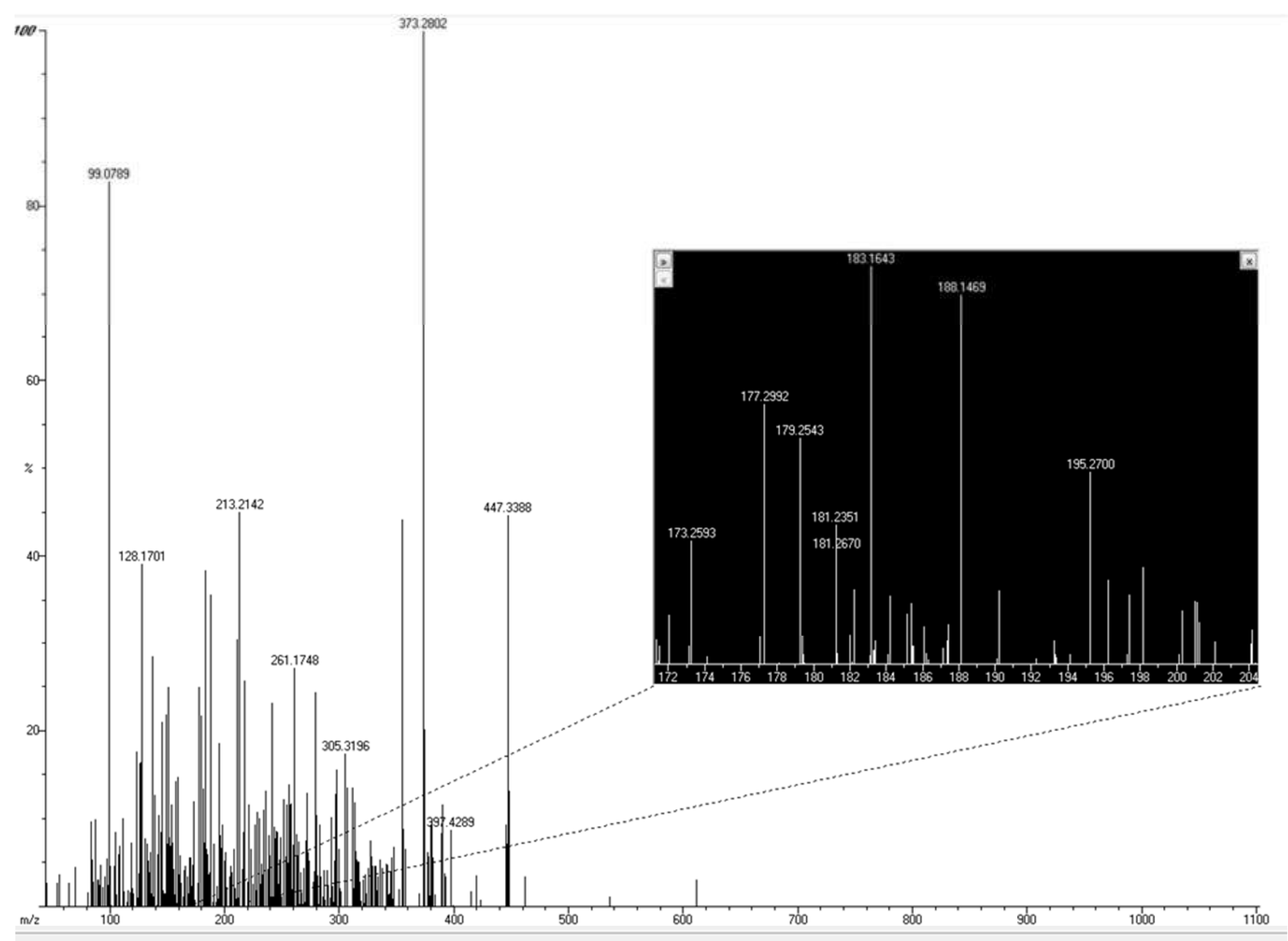

Figure 58 - Mass spectrum of 2,4-dinitrotoluene in positive ion mode at $350^{\circ} \mathrm{C}(30 \mathrm{~V})$.

When running 2,4-DNT in negative ion mode, it was equally problematic. 2,4-DNT has a molecular mass of $182.13 \mathrm{~g} / \mathrm{mol}([\mathrm{M}]=182.13 \mathrm{~m} / \mathrm{z})$. Negative ion mode deprotonates to create $\mathrm{M}-\mathrm{H}=181.12 \mathrm{~m} / \mathrm{z}$. However, when 2,4-DNT was sampled in negative ion mode, it resulted in a parent ion peak of $182.99 \mathrm{~m} / \mathrm{z}$ (Figure 59).

The instrument was calibrated properly in positive ion mode and the possibility of calibration drift was ruled out. After numerous trials, it was decided that 2,4-DNT would be omitted for this research and replaced with another explosive odorant. For this, odorants for smokeless powders were attempted based on research conducted by Joshi et. Al [54]. 


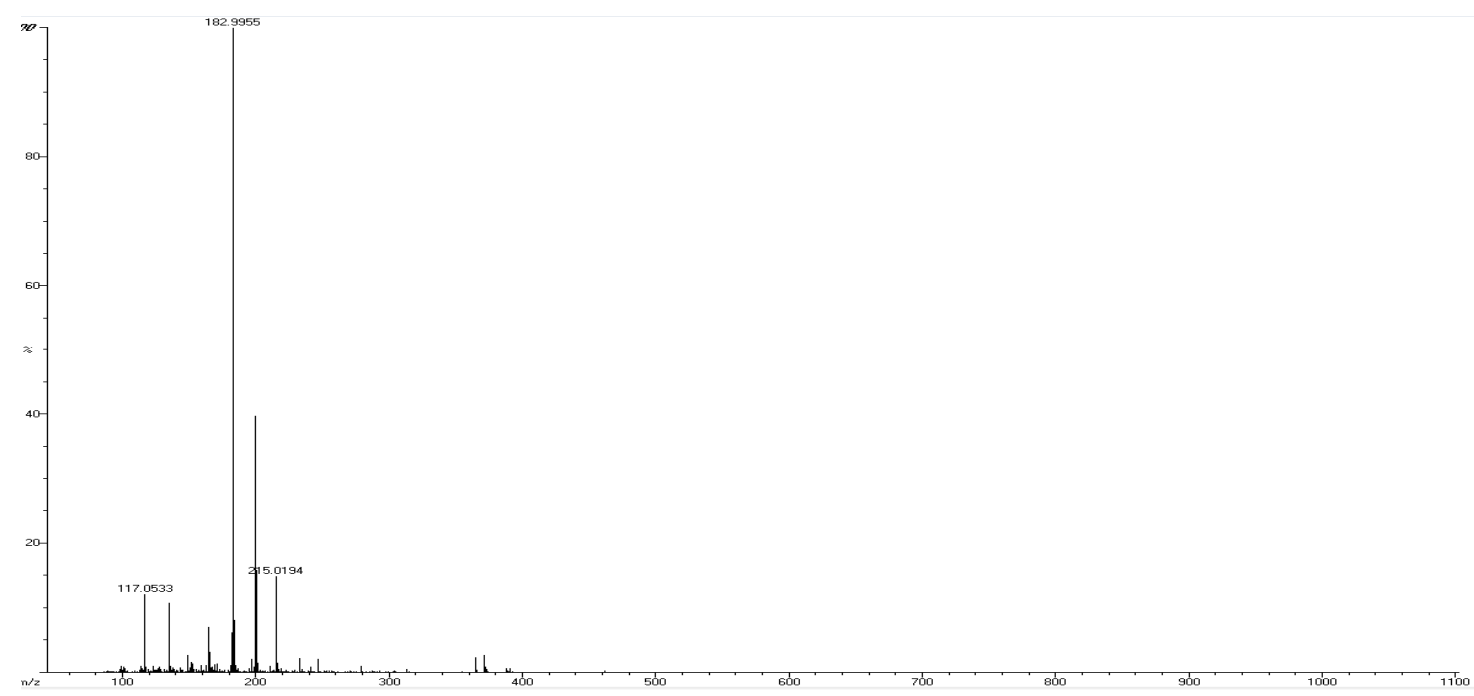

Figure 59 - Mass spectrum of 2,4-dinitrotoluene in negative ion mode at $350^{\circ} \mathrm{C}(30 \mathrm{~V})$.

As indicated in the paper, diphenylamine (DPA), ethyl centralite (EC), methyl centralite (MC), 2,4-dinitrotoluene (2,4-DNT), diethyl phthalate (DEP) and dibutyl phthalate (DBP) are consistently observed in the headspace of most smokeless powders. 2,4-DNT has already been discussed and omitted. Both ethyl and methyl centralite standards are in crystalline powder form, and in effort to keep things consistent with this study they, too, were omitted. Despite DPA being the most prevalent chemical in the headspace of smokeless powders, sampling its headspace using DART-AccuTOF did not produce reproducible results. Sampling the liquid directly did produce positive results, but this research is focused on headspace analysis.

This leaves DEP and DBP as the remaining candidates to replace 2,4-DNT. Although the headspace of both samples did produce results, DBP was relatively extremely weak in its response (Figure 60), and at lower temperatures it was not detected at all as shown in Figure 61 (Table 11). Therefore, diethyl phthalate was chosen for the remainder of this research. 
Table 11 - Average response of dibutyl phthalate (DBP) and diethyl phthalate (DEP) at temperatures ranging from $150^{\circ} \mathrm{C}-500^{\circ} \mathrm{C}$.

\begin{tabular}{|c|c|c|c|}
\hline \multicolumn{2}{|c|}{ DBP } & \multicolumn{2}{c|}{ DEP } \\
\hline Temperature & Response $(x 1000)$ & Temperature & Response $(x 1000)$ \\
\hline $150{ }^{\circ} \mathrm{C}$ & No response & $150{ }^{\circ} \mathrm{C}$ & 50.4 \\
\hline $200^{\circ} \mathrm{C}$ & No response & $200^{\circ} \mathrm{C}$ & 34.6 \\
\hline $250^{\circ} \mathrm{C}$ & 5.8 & $250{ }^{\circ} \mathrm{C}$ & 15.2 \\
\hline $300^{\circ} \mathrm{C}$ & 4.6 & $300^{\circ} \mathrm{C}$ & 89.5 \\
\hline $350^{\circ} \mathrm{C}$ & 5 & $350{ }^{\circ} \mathrm{C}$ & 37.4 \\
\hline $400^{\circ} \mathrm{C}$ & 3 & $400^{\circ} \mathrm{C}$ & 113.5 \\
\hline $450^{\circ} \mathrm{C}$ & 6 & $450^{\circ} \mathrm{C}$ & 150.1 \\
\hline $500^{\circ} \mathrm{C}$ & 14 & $500^{\circ} \mathrm{C}$ & 159.1 \\
\hline
\end{tabular}

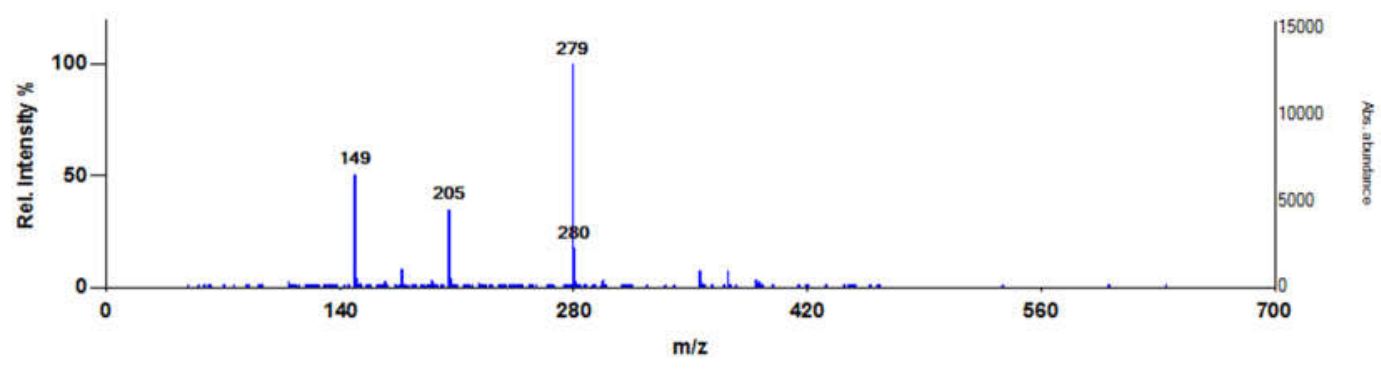

Figure 60 - Mass spectrum for dibutyl phthalate with molecular ion peak $(279 \mathrm{~m} / \mathrm{z})$ present but at a very low intensity $\left(\right.$ at $\left.500^{\circ} \mathrm{C}\right)$.

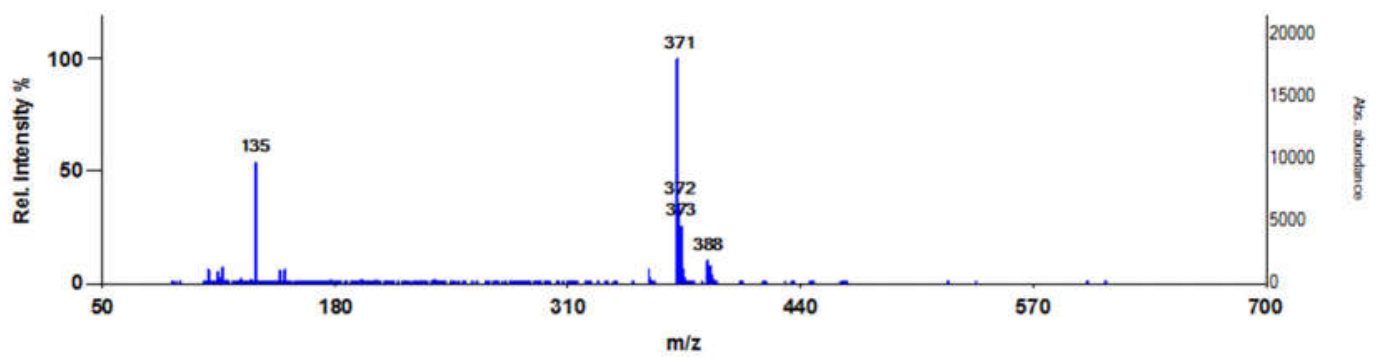

Figure 61 - Mass spectrum for dibutyl phthalate where the molecular ion peak $(279 \mathrm{~m} / \mathrm{z})$ was not observed (at $\left.200^{\circ} \mathrm{C}\right)$. 

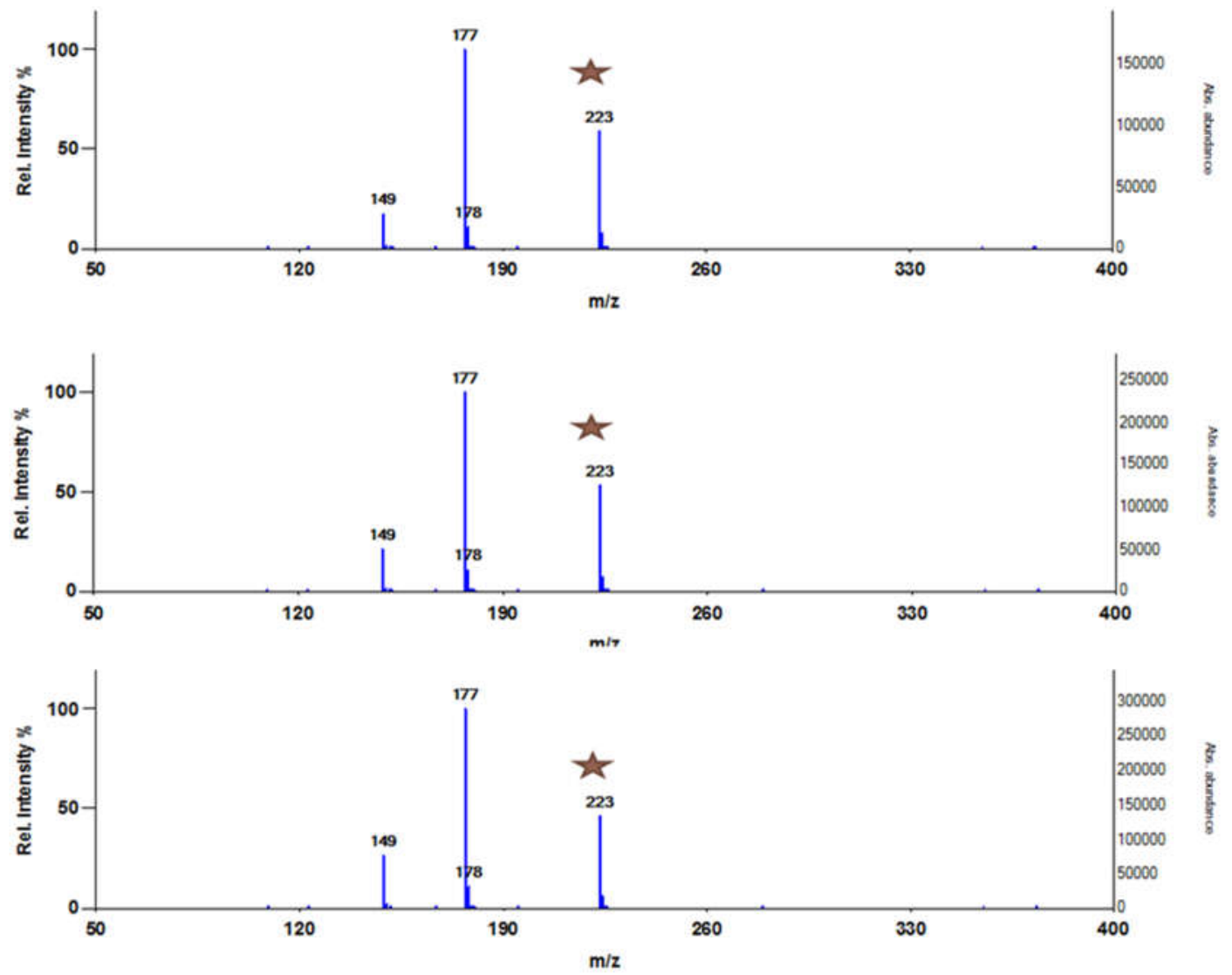

Figure 62 - Mass spectra of diethyl phthalate at $400^{\circ} \mathrm{C}$ (top), $450^{\circ} \mathrm{C}$ (middle) and $500^{\circ} \mathrm{C}$ (bottom).

After all the samples were analyzed at the various temperatures, the process was repeated two more times and the averages were calculated (Table 12). The purpose of this step is to determine the optimal temperature to be used for the remainder of this research. When plotting that data into a graph (Figure 63), it can be observed that $400^{\circ} \mathrm{C}$ produces the best results for 2-ethyl-1-hexanol, while still producing great results for the remaining three standards that are within the same order of magnitude. 
Table 12 - Averaged responses (x1000) for the four standards at temperatures $150^{\circ}-500^{\circ} \mathrm{C}$ at $50^{\circ} \mathrm{C}$ intervals.

\begin{tabular}{|cc|c|c|c|}
\hline & Methyl Benzoate & \multicolumn{1}{c}{ Benzaldehyde } & 2-ethyl-1-hexanol & Diethyl Pthalate \\
\hline $150^{\circ} \mathrm{C}$ & 399 & 339 & 72 & 279 \\
$200^{\circ} \mathrm{C}$ & 397 & 337 & 63 & 304 \\
$250^{\circ} \mathrm{C}$ & 392 & 287 & 60 & 344 \\
$300^{\circ} \mathrm{C}$ & 358 & 350 & 51 & 388 \\
\hline $350^{\circ} \mathrm{C}$ & 323 & 350 & 104 & 373 \\
$400^{\circ} \mathrm{C}$ & 395 & 377 & 138 & 392 \\
$450^{\circ} \mathrm{C}$ & 394 & 380 & 60 & 395 \\
$500^{\circ} \mathrm{C}$ & 332 & 323 & 45 & 330 \\
\hline
\end{tabular}

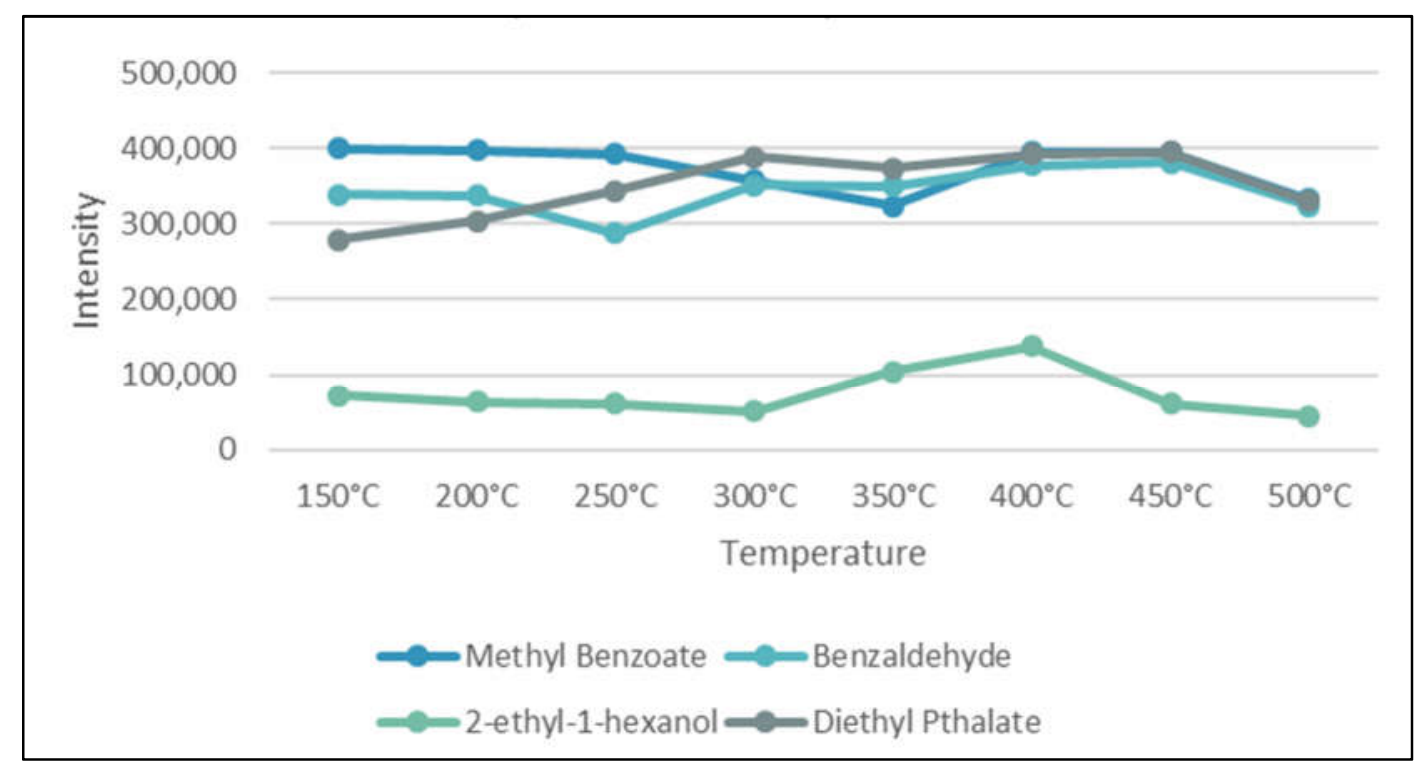

Figure 63 - Visual representation of the data from Table 12 - Averaged responses (x1000) for the four standards at temperatures $150^{\circ}-500^{\circ} \mathrm{C}$ at $50^{\circ} \mathrm{C}$ intervals.. 


\subsection{Task 2: Evaluating the Speed of VOC Transport}

Four chemical standards were tested at five varying distances $(0.5 \mathrm{~m}, 1 \mathrm{~m}, 1.5 \mathrm{~m}, 2 \mathrm{~m}$, and $2.5 \mathrm{~m}$ ) from the DART source. Each test was done in triplicate resulting in 60 data sets. The layout for the room can be seen in Figure 32. Calibration using PEG 600 was done for each run, and each run ends with the use of a positive control. For each run, three main graphs were collected when possible. The RIC of the total run (after performing CODA), RIC of the run after selecting the $m / z$ of choice, and the mass spectrum at the point in time at which the sample of interest is first identified.

\section{Methyl Benzoate}

Figure 64 shows the RIC (after performing CODA) for the first trial testing of methyl benzoate at a distance of $0.5 \mathrm{~m}$. The RIC shows the PEG peaks in the beginning of the run and the direct sampling of the methyl benzoate (positive control) towards the end. By selecting the m/z of 137 and creating a chromatogram (as shown in Figure 33) that will show a RIC where only m/z 137 was identified, the moment in which the instrument first detects the volatile organic compound can be determined (Figure 65).

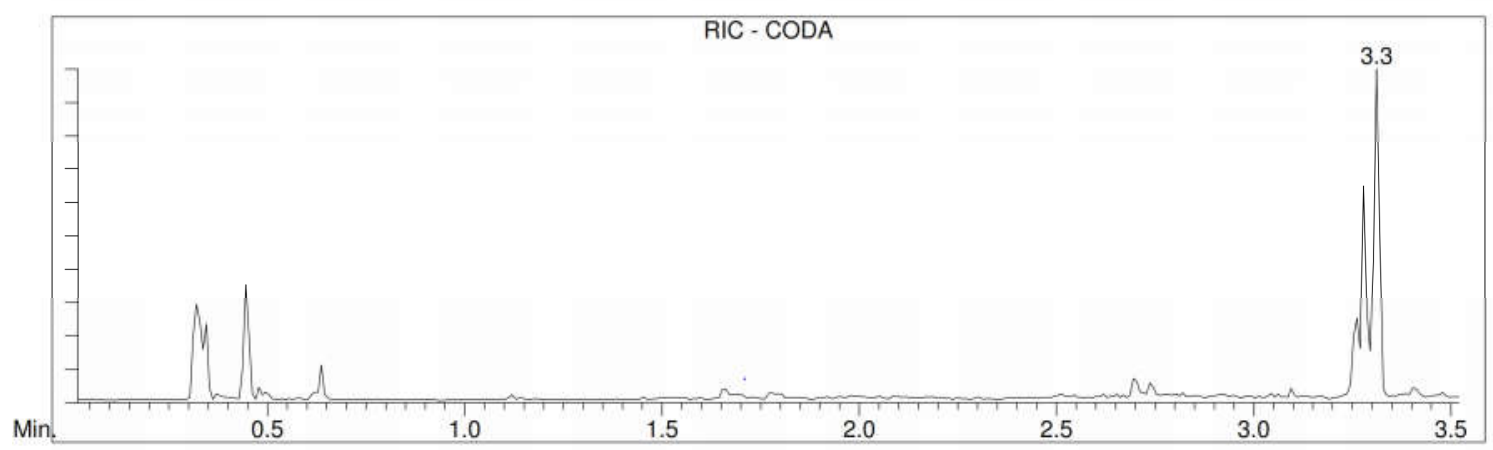

Figure 64 - RIC of methyl benzoate (trial \#1) at a distance of $0.5 \mathrm{~m}$. 


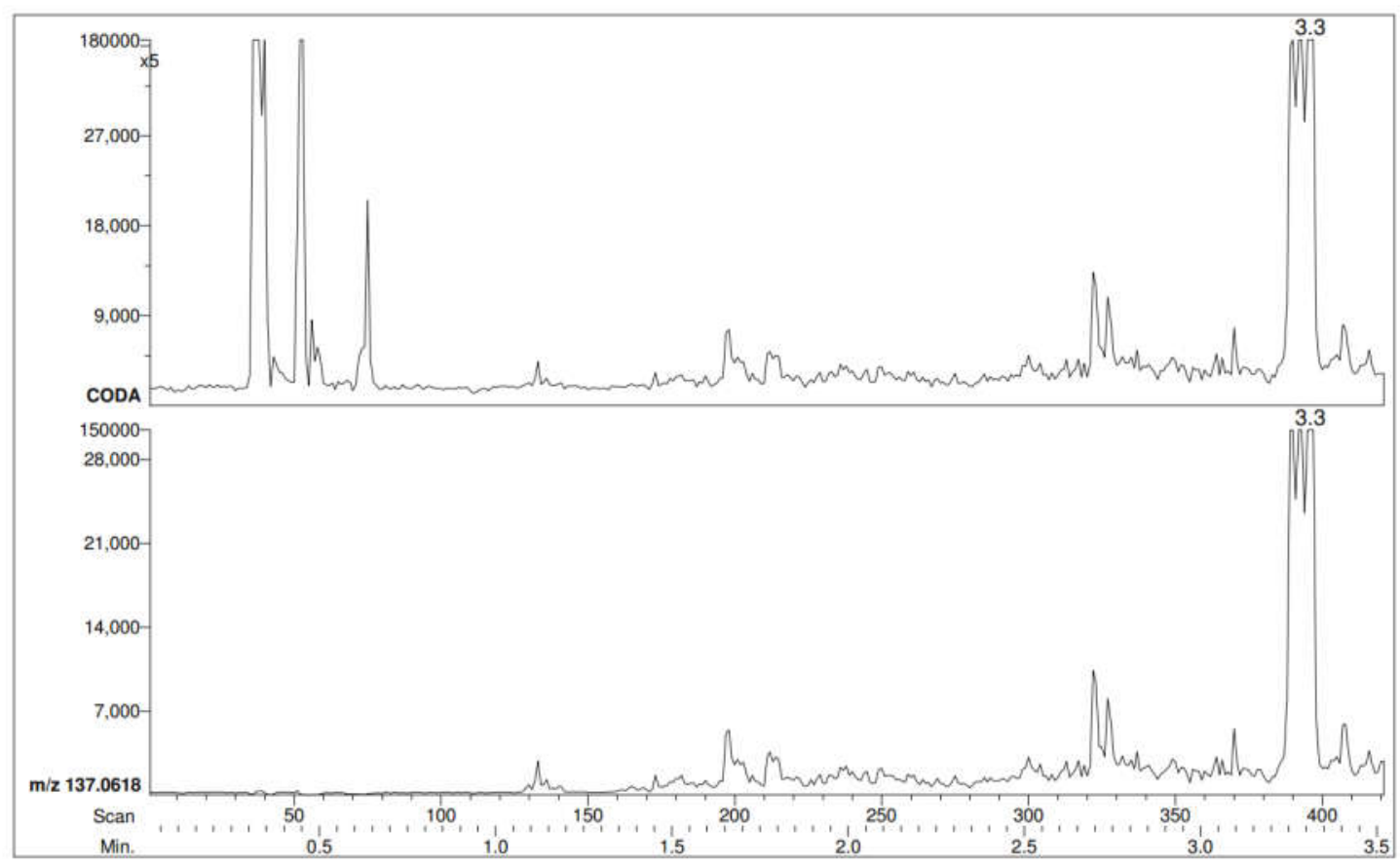

Figure 65 - RIC's of methyl benzoate at a distance of $0.5 \mathrm{~m}$ (Trial \#1). Top: RIC after performing CODA (zoomed in). Bottom: RIC showing only peaks where $137 \mathrm{~m} / \mathrm{z}$ was detected. Cap opened at 1:02 minutes.

When looking at the RIC created for $137 \mathrm{~m} / \mathrm{z}$, the next step is to identify the moment where the sample was first detected, keeping in mind that the molar mass identified must fall within $5 \mathrm{mDa}$ of the calculated $\mathrm{m} / \mathrm{z}$ of the sample, as well as the molecular ion peak being above $5 \%$ in relative intensity.

Figure 66 shows the mass spectrum and time (RT = 'retention time') where methyl benzoate was first detected. It is important to note that since this is not a chromatographic technique, retention time is technically incorrect, but it is what the software uses to identify the moment in which a sample is detected. For trial \#1, the vial containing methyl benzoate was opened at 1:02 (1 minute and 2 seconds) from when the run began and was first identified 5 seconds later (at 1:07 or 1.12). 


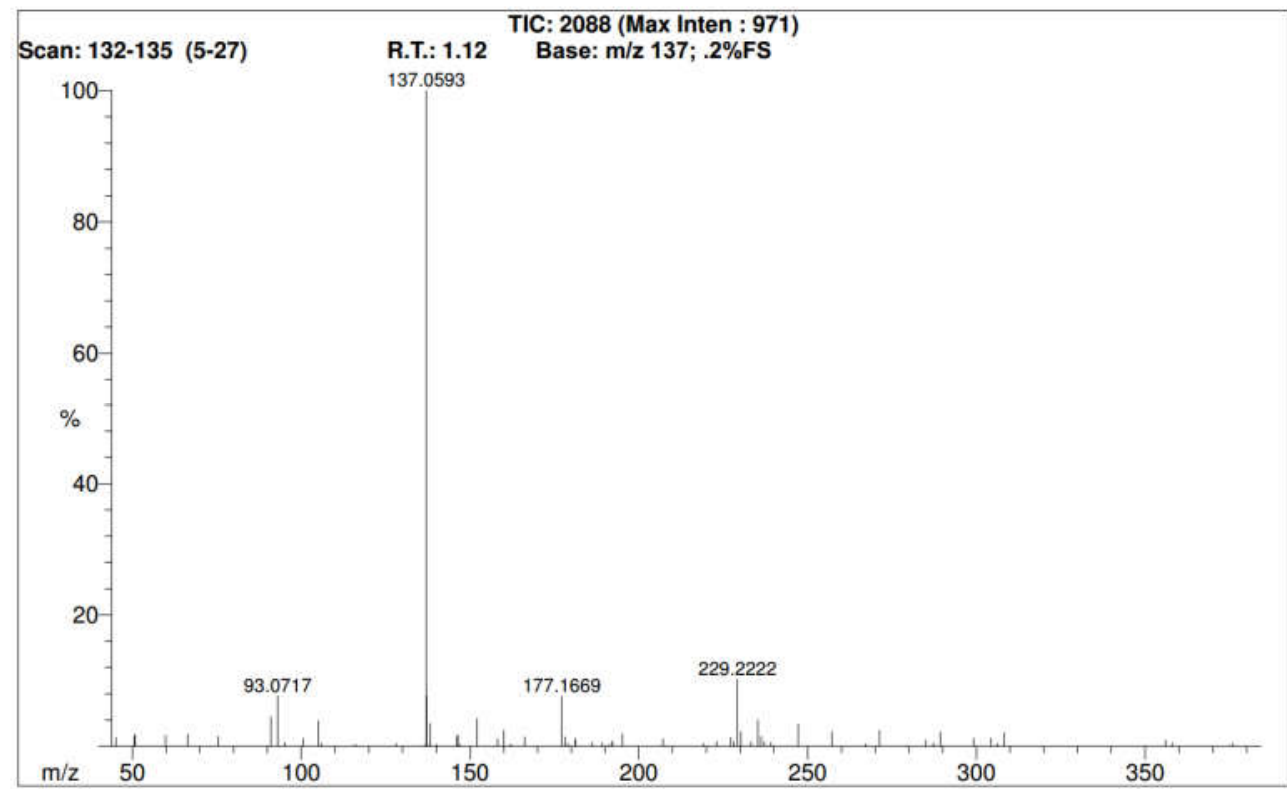

Figure 66 - Mass spectrum of methyl benzoate (trial \#1) at distance $0.5 \mathrm{~m}$, first detected at 1.12 minutes.

In trial \#2, the RIC's are almost identical (Figure 67), and while the cap was opened at the same time (1:02min), methyl benzoate was first detected 8 seconds later (1:10 or $1.18 \mathrm{~min}$ ) as shown in the mass spectrum in Figure 68. 


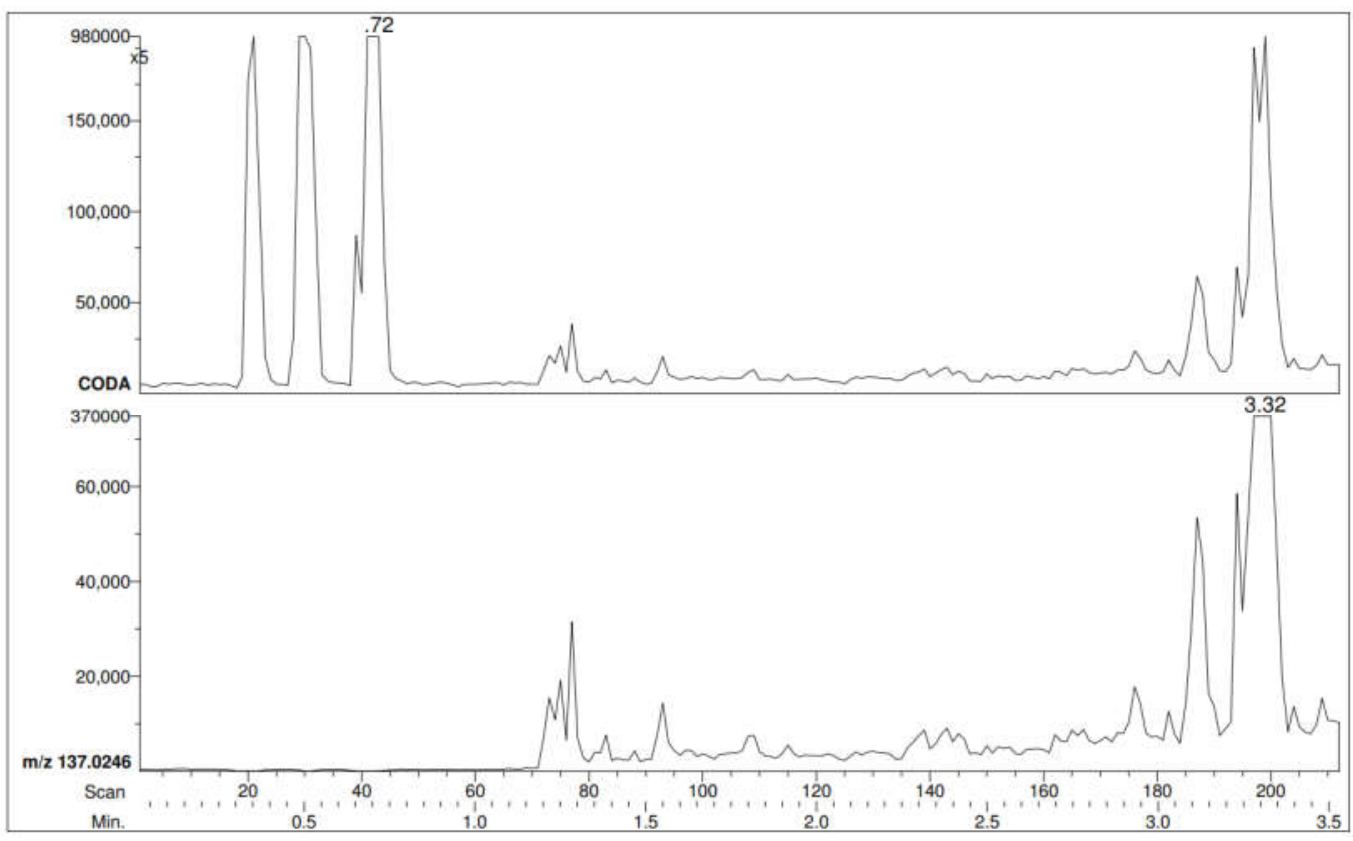

Figure 67 - RIC's of methyl benzoate at a distance of $0.5 \mathrm{~m}$ (Trial \#2). Top: RIC after performing CODA (zoomed in). Bottom: RIC showing only peaks where $137 \mathrm{~m} / \mathrm{z}$ was detected. Cap opened at 1:02 minutes.

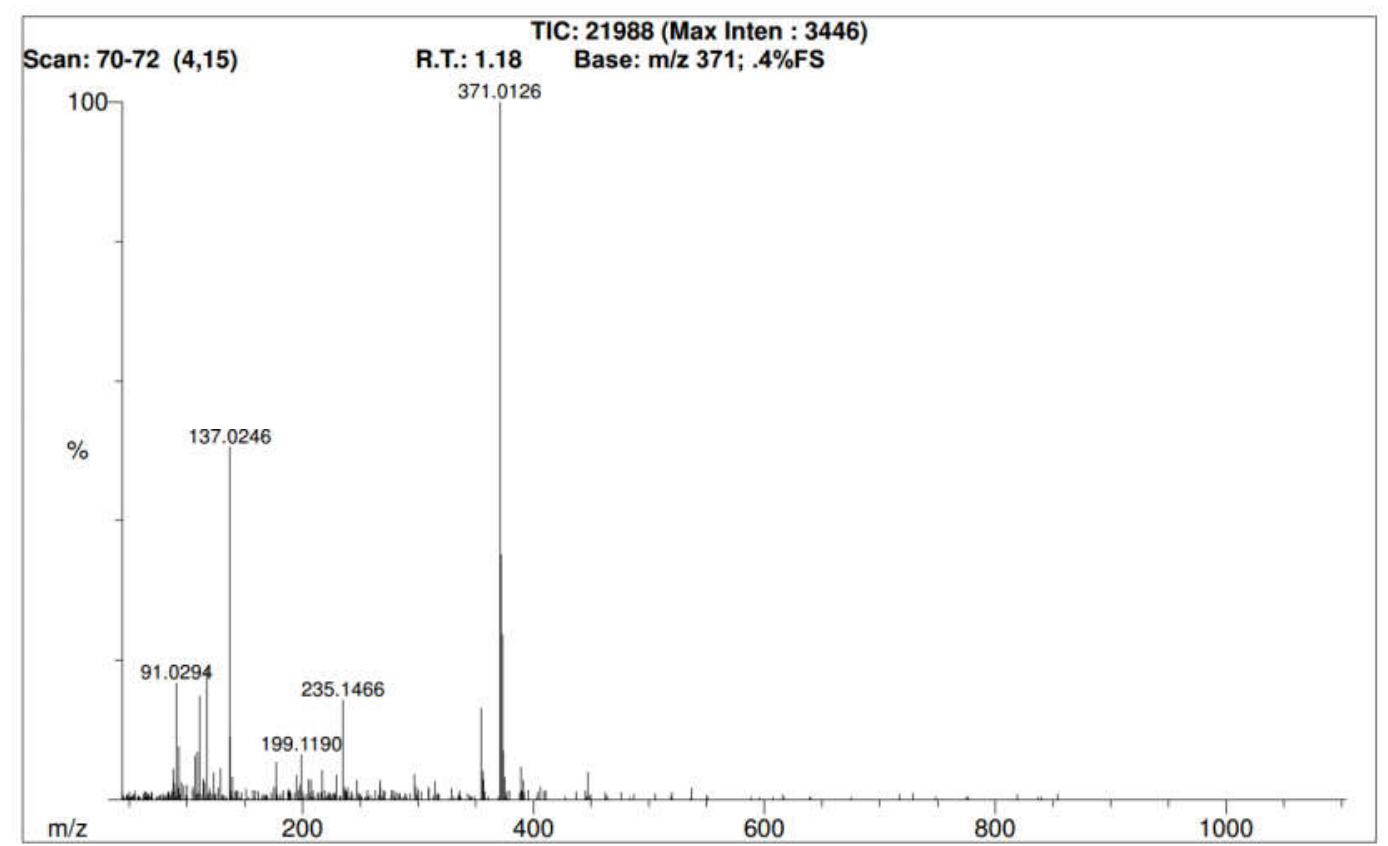

Figure 68 - Mass spectrum of methyl benzoate (trial \#2) at distance $0.5 \mathrm{~m}$, first detected at $1.18(1: 10)$ minutes. 
For the third trial at this distance for methyl benzoate, the cap was opened at 1:03 and was identified five seconds later at 1:08 (or 1.13) minutes (Figure 69 and Figure 70). Table 13 summarizes the results for the three trials.

Table 13 - Summary of results for methyl benzoate at $0.5 \mathrm{~m}$.

\begin{tabular}{|c|c|c|c|c|}
\hline \multicolumn{5}{|c|}{ Methyl Benzoate at $0.5 m\left(400^{\circ} \mathrm{C}\right)$} \\
\hline Trial & Vial Open & Vial Closed & Detected & Time (s) \\
\hline$\# 1$ & $1: 02$ & $3: 08$ & $1: 07$ & 5 \\
\hline$\# 2$ & $1: 02$ & $3: 05$ & $1: 10$ & 8 \\
\hline$\# 3$ & $1: 03$ & $3: 06$ & $1: 08$ & 5 \\
\hline
\end{tabular}

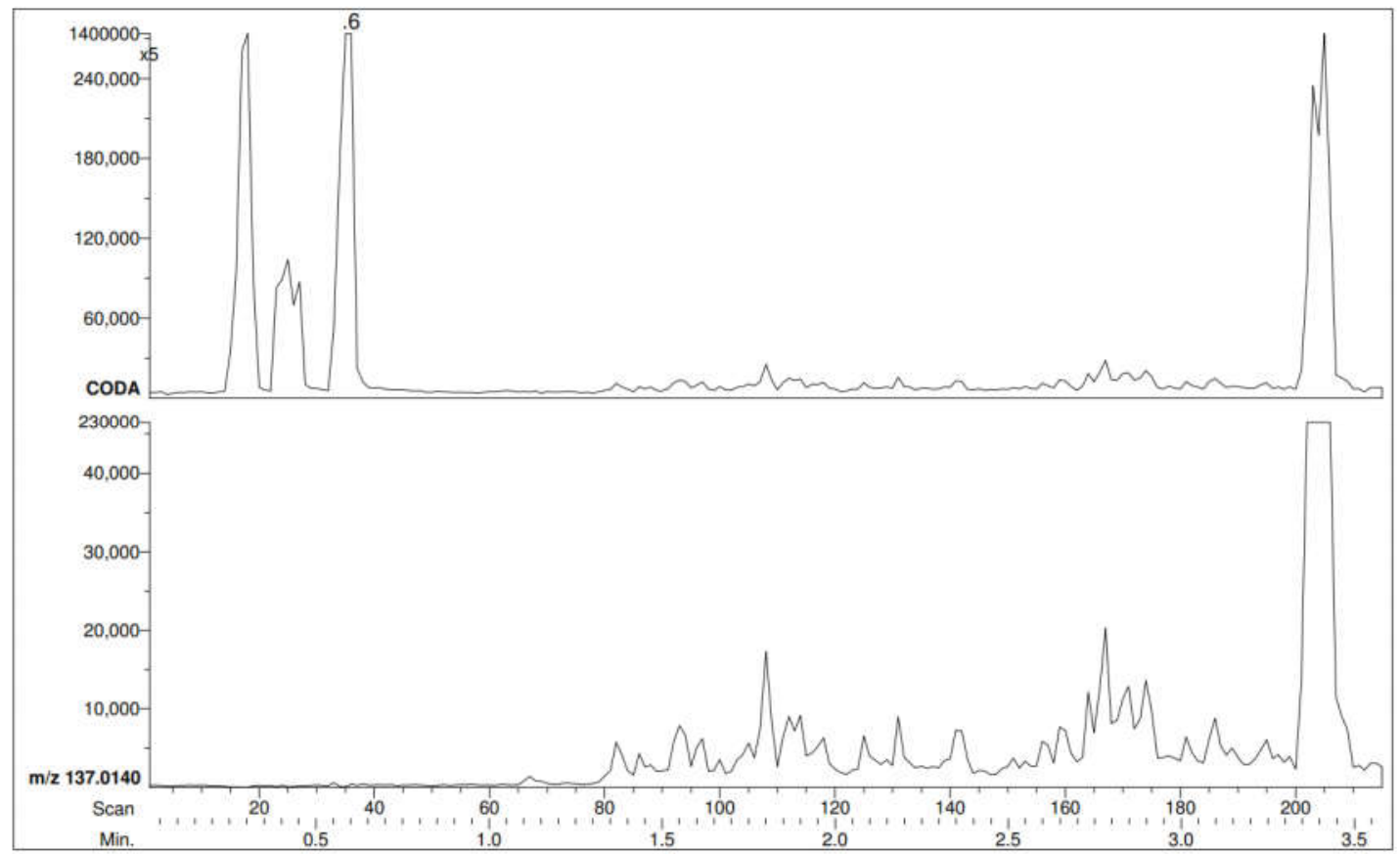

Figure 69 - RIC's of methyl benzoate at a distance of $0.5 \mathrm{~m}$ (Trial \#2). Top: RIC after performing CODA (zoomed in). Bottom: RIC showing only peaks where $137 \mathrm{~m} / \mathrm{z}$ was detected. Cap opened at 1:03 minutes. 


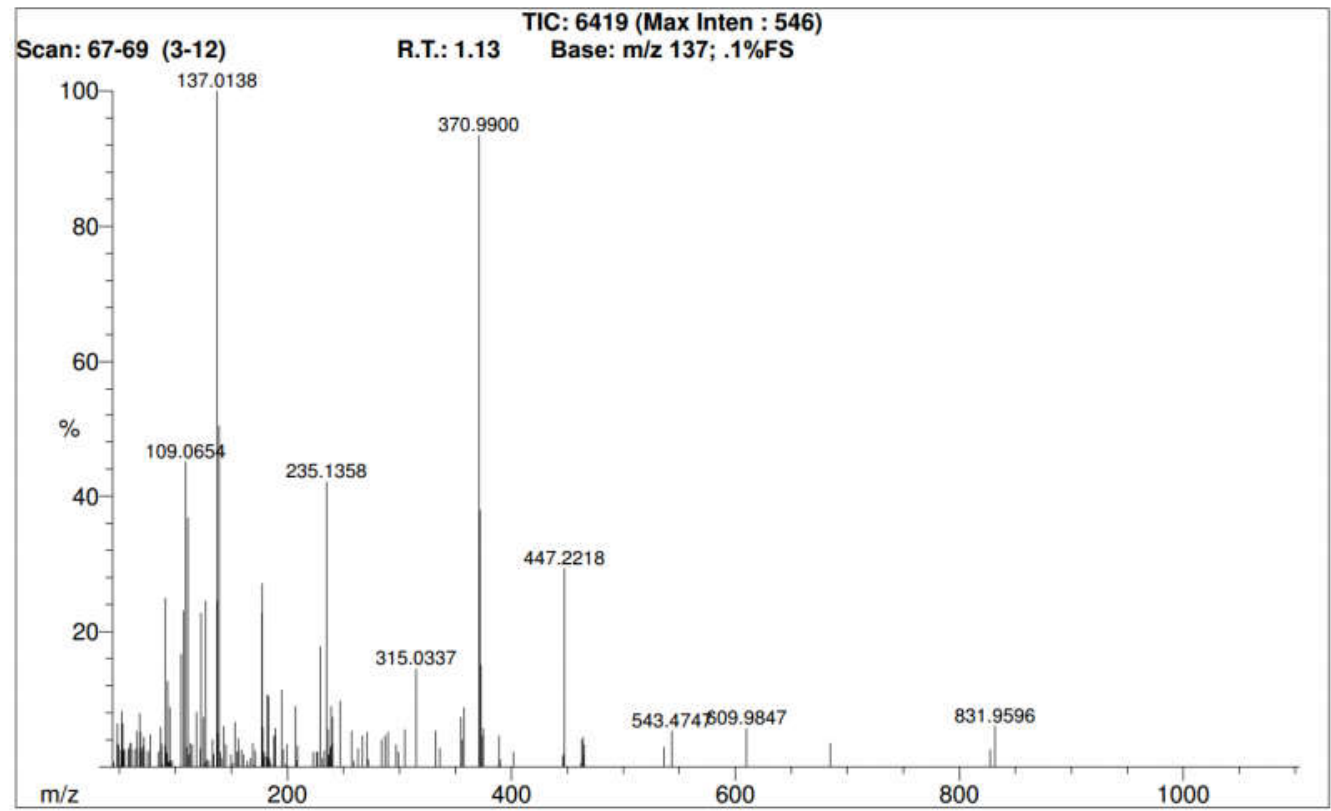

Figure 70 - Mass spectrum of methyl benzoate (trial \#3) at distance $0.5 \mathrm{~m}$, first detected at $1.13(1: 08)$ minutes.

Next, methyl benzoate was tested at a distance of $1 \mathrm{~m}$. For the first trial, the vial cap was opened 56 seconds after the run was started (Figure 71). For the second trial, the cap was opened after 1 minute (Figure 73), while for the third trial, it was opened after 1 minute and 1 second (Figure 75). The mass spectra for those trials are shown in Figure 72, Figure 74, and Figure 76. The summary of these runs are shown in Table 14 - Summary of results for methyl benzoate at $1 \mathrm{~m}$.

Table 14 - Summary of results for methyl benzoate at $1 \mathrm{~m}$.

\begin{tabular}{|c|c|c|c|c|}
\hline \multicolumn{5}{|c|}{ Methyl Benzoate at $1 \mathrm{~m}\left(400^{\circ} \mathrm{C}\right)$} \\
\hline Trial & Vial Open & Vial Closed & Detected & Time (s) \\
\hline$\# 1$ & $0: 56$ & $3: 01$ & $1: 11$ & 15 \\
\hline$\# 2$ & $1: 00$ & $3: 03$ & $1: 22$ & 22 \\
\hline$\# 3$ & $1: 01$ & $3: 04$ & $1: 24$ & 23 \\
\hline
\end{tabular}




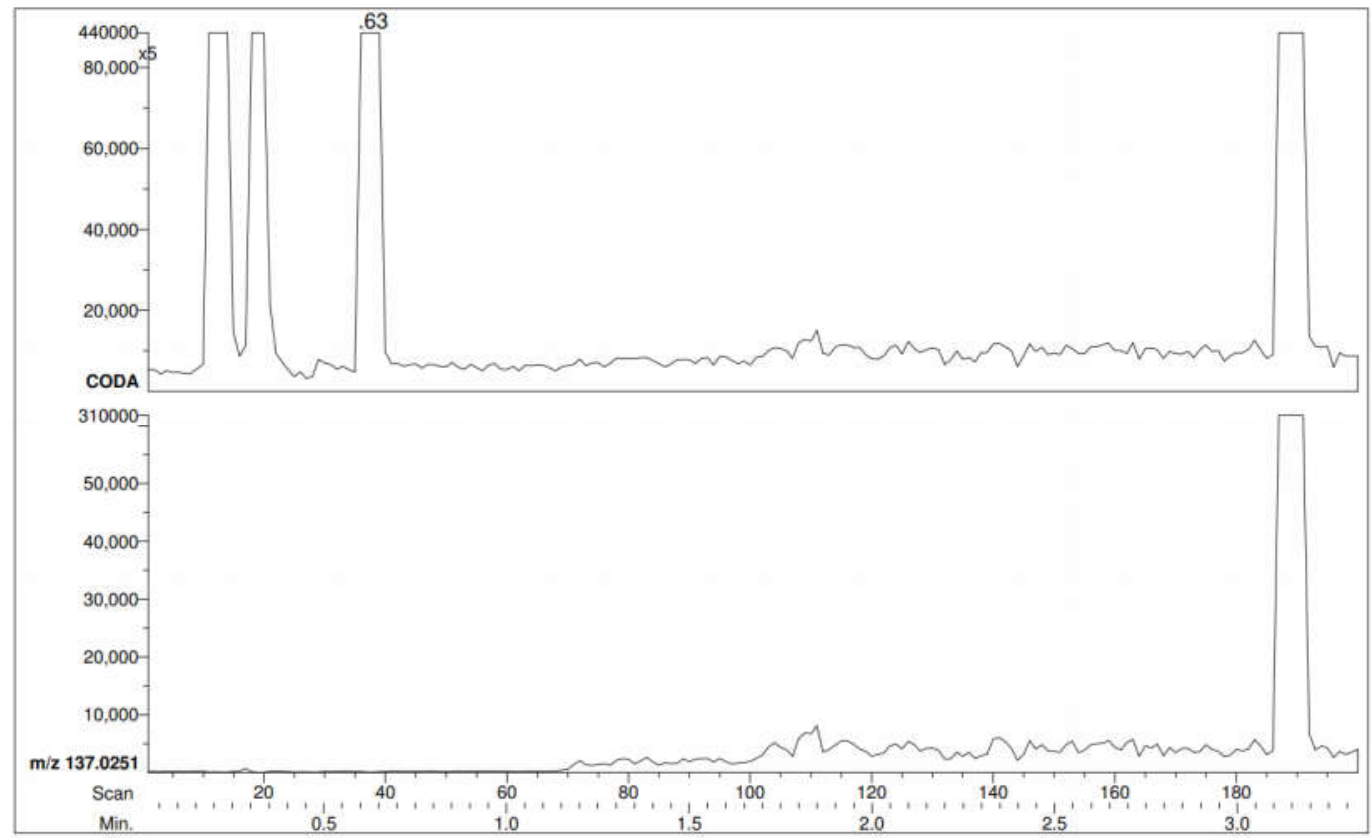

Figure 71 - RIC's of methyl benzoate at a distance of $1 \mathrm{~m}$ (Trial \#1). Top: RIC after performing CODA (zoomed in). Bottom: RIC showing only peaks where $137 \mathrm{~m} / \mathrm{z}$ was detected. Cap opened at 56 seconds.

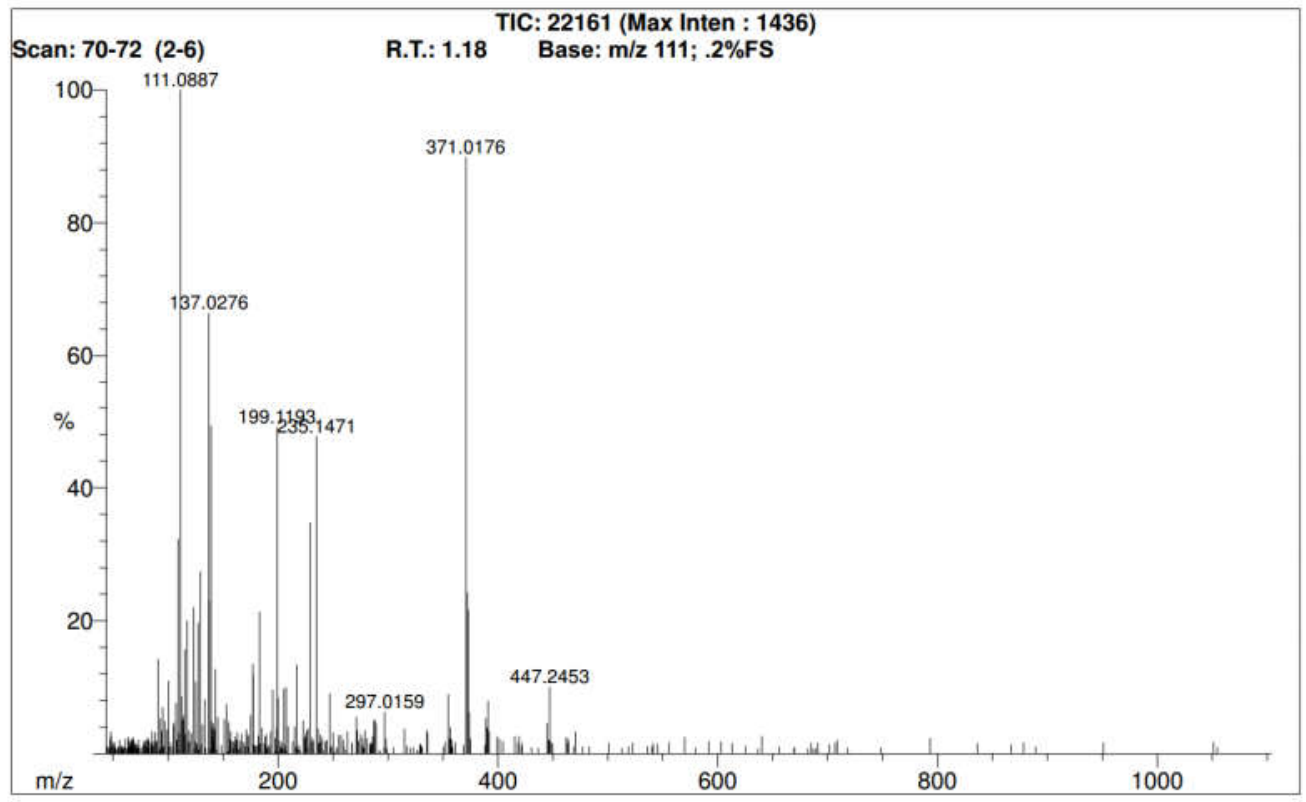

Figure 72 - Mass spectrum of methyl benzoate (trial \#1) at distance 1m, first detected at $1.18(1: 11)$ minutes. 


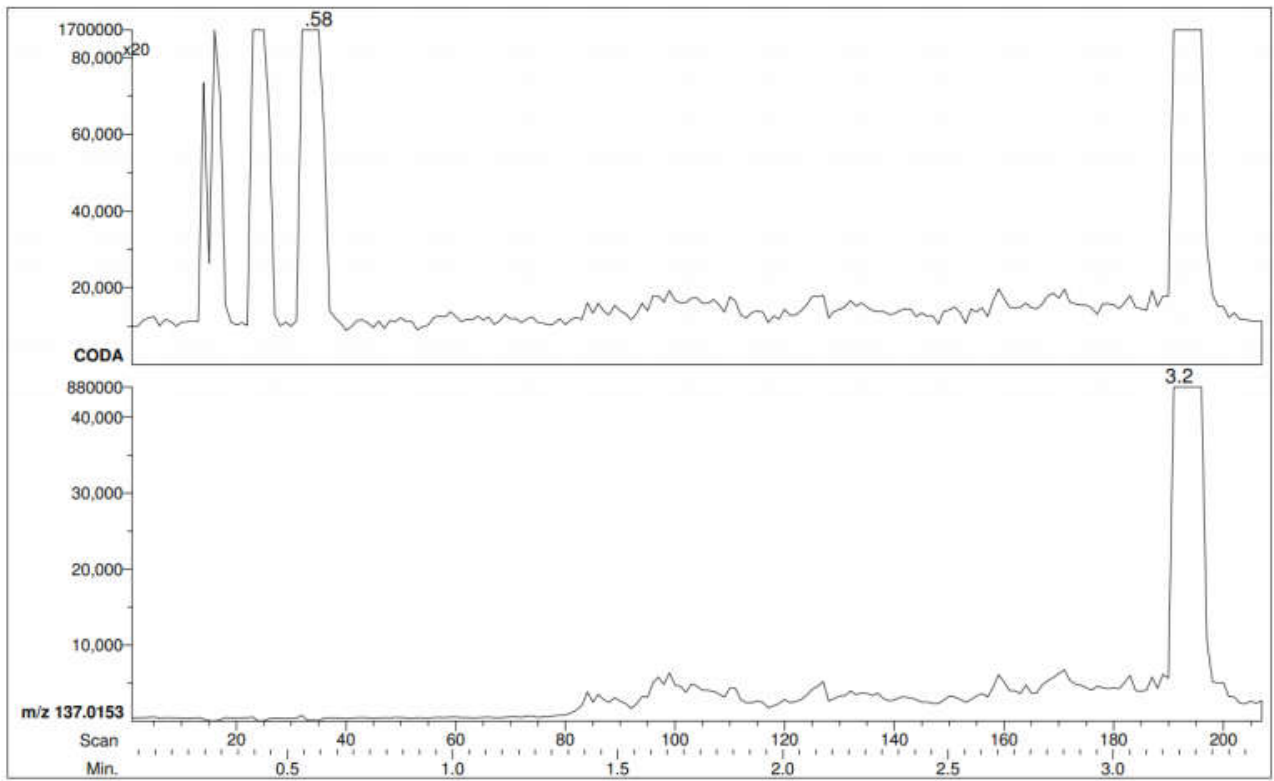

Figure 73 - RIC's of methyl benzoate at a distance of 1m (Trial \#2). Top: RIC after performing CODA (zoomed in). Bottom: RIC showing only peaks where $137 \mathrm{~m} / \mathrm{z}$ was detected. Cap opened at 1 minute.

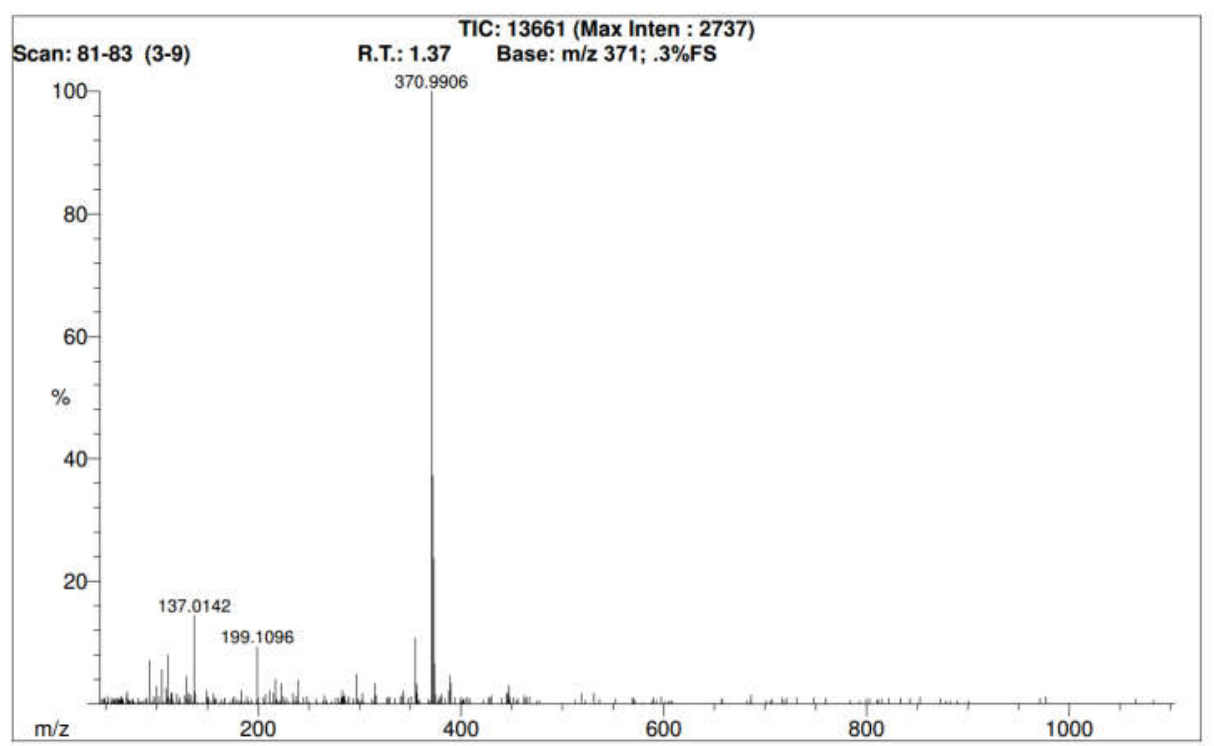

Figure 74 - Mass spectrum of methyl benzoate (trial \#2) at distance 1m, first detected at $1.37(1: 22)$ minutes. 


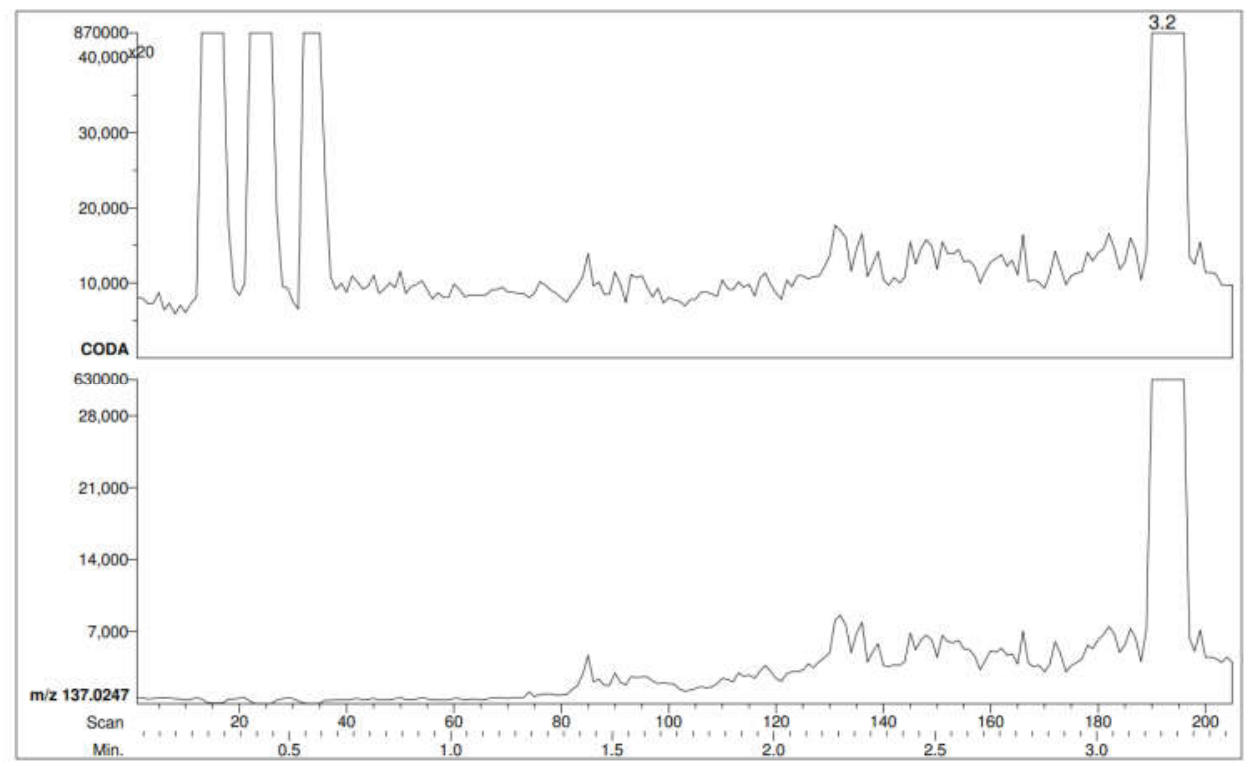

Figure 75 - RIC's of methyl benzoate at a distance of $1 \mathrm{~m}$ (Trial \#3). Top: RIC after performing CODA (zoomed in). Bottom: RIC showing only peaks where $137 \mathrm{~m} / \mathrm{z}$ was detected. Cap opened at 1 minute and 1 second (1:01).

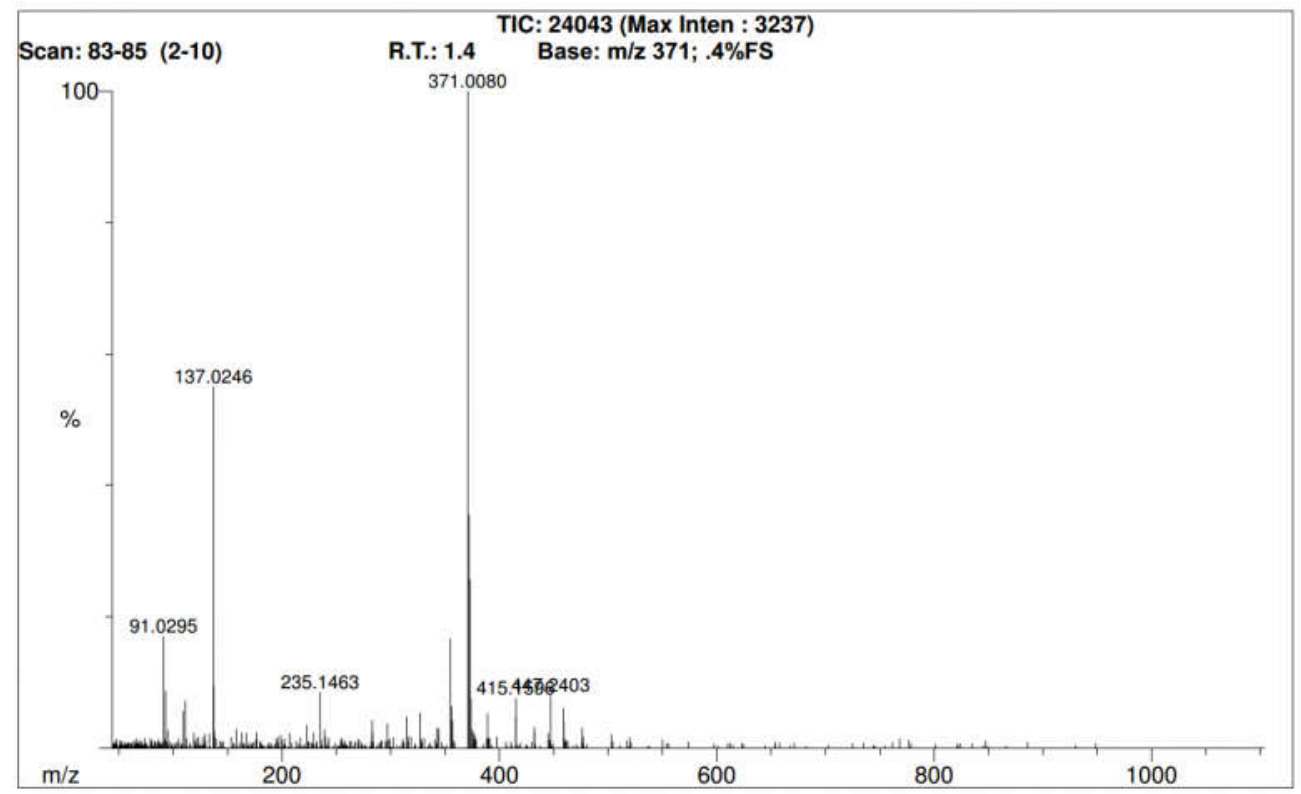

Figure 76 - Mass spectrum of methyl benzoate (trial \#3) at distance 1m, first detected at 1.4 $(1: 24)$ minutes.

Results for methyl benzoate tested at distances $1.5 \mathrm{~m}, 2 \mathrm{~m}$ and $3 \mathrm{~m}$ are summarized in Table 15, Table 16 and Table 17 respectively. 
Table 15 - Summary of results for methyl benzoate at a distance of $1.5 \mathrm{~m}$.

\begin{tabular}{|c|c|c|c|c|}
\hline \multicolumn{5}{|c|}{ Methyl Benzoate at $1.5 m\left(400^{\circ} \mathrm{C}\right)$} \\
\hline Trial & Vial Open & Vial Closed & Detected & Time (s) \\
\hline$\# 1$ & $1: 04$ & $3: 08$ & $1: 26$ & 22 \\
\hline$\# 2$ & $0: 50$ & $2: 54$ & $1: 14$ & 24 \\
\hline$\# 3$ & $1: 32$ & $3: 17$ & $1: 56$ & 24 \\
\hline
\end{tabular}

Table 16 - Summary of results for methyl benzoate at a distance of $2 \mathrm{~m}$.

\begin{tabular}{|c|c|c|c|c|}
\hline \multicolumn{5}{|c|}{ Methyl Benzoate at $2 \mathrm{~m}\left(400^{\circ} \mathrm{C}\right)$} \\
\hline Trial & Vial Open & Vial Closed & Detected & Time (s) \\
\hline$\# 1$ & $1: 10$ & $3: 12$ & $1: 41$ & 31 \\
\hline$\# 2$ & $1: 01$ & $3: 04$ & $1: 30$ & 29 \\
\hline$\# 3$ & $1: 10$ & $3: 15$ & $1: 43$ & 33 \\
\hline
\end{tabular}

Table 17 - Summary of results for methyl benzoate at a distance of $3 \mathrm{~m}$.

\begin{tabular}{|c|c|c|c|c|}
\hline \multicolumn{5}{|c|}{ Methyl Benzoate at $3 \mathrm{~m}\left(400^{\circ} \mathrm{C}\right)$} \\
\hline Trial & Vial Open & Vial Closed & Detected & Time (s) \\
\hline$\# 1$ & $1: 16$ & $3: 20$ & $1: 53$ & 37 \\
\hline$\# 2$ & $1: 31$ & $3: 33$ & $2: 09$ & 38 \\
\hline$\# 3$ & $1: 09$ & $3: 12$ & $1: 52$ & 43 \\
\hline
\end{tabular}

To illustrate the results for methyl benzoate at these various distances, the averages of all trials were plotted and their standard deviations accounted for in the form of error bars (Figure 77). From this graph, it can be seen that it takes longer to detect the sample as the distance from the source increases. 


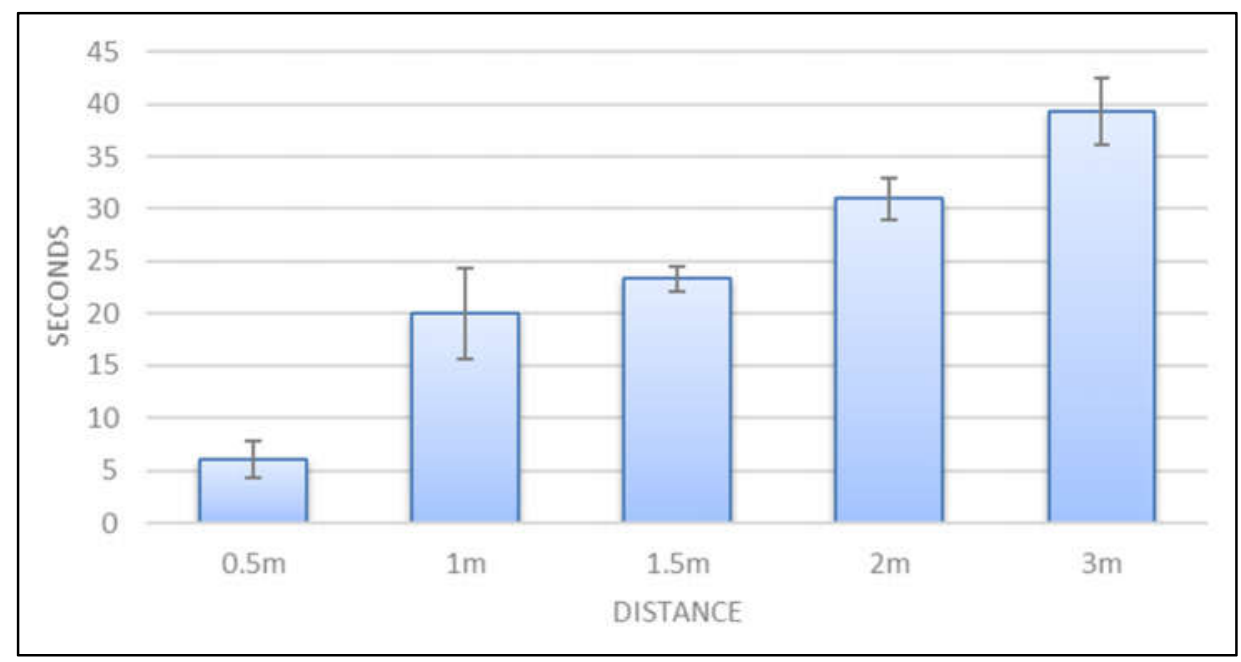

Figure 77 - Averaged results of task 2 study for methyl benzoate at the various distances.

\section{Benzaldehyde}

With benzaldehyde, the results were similar in that it was detected at each distance for all trials. Table 18 shows all the data for benzaldehyde at distances $0.5 \mathrm{~m}$ through $3 \mathrm{~m}$. Figure 78 and Figure 79 shows an example RIC and mass spectrum, respectively, for the second trial of benzaldehyde at a distance of $2 \mathrm{~m}$. Again, Figure 78 illustrates the manner in which the volatiles detected increase in intensity as the time increases. In this particular example, it took 44 seconds from the moment the vial cap was opened for benzaldehyde to be detected initially. 


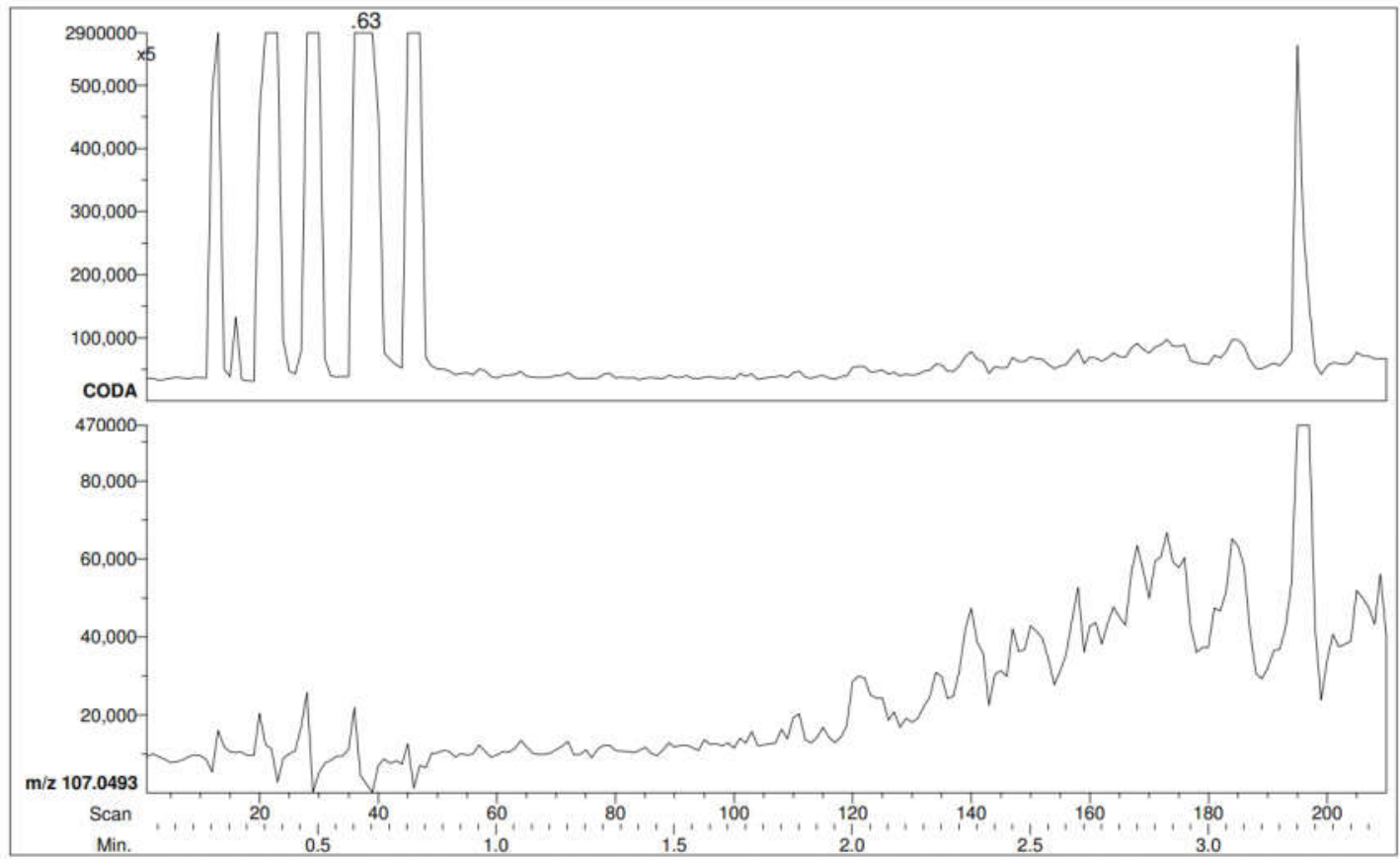

Figure 78 - RIC's of benzaldehyde at a distance of $2 \mathrm{~m}$ (Trial \#2). Top: RIC after performing CODA (zoomed in). Bottom: RIC showing only peaks where $107 \mathrm{~m} / \mathrm{z}$ was detected. Cap opened at 1 minute and 6 seconds (1:06 min).

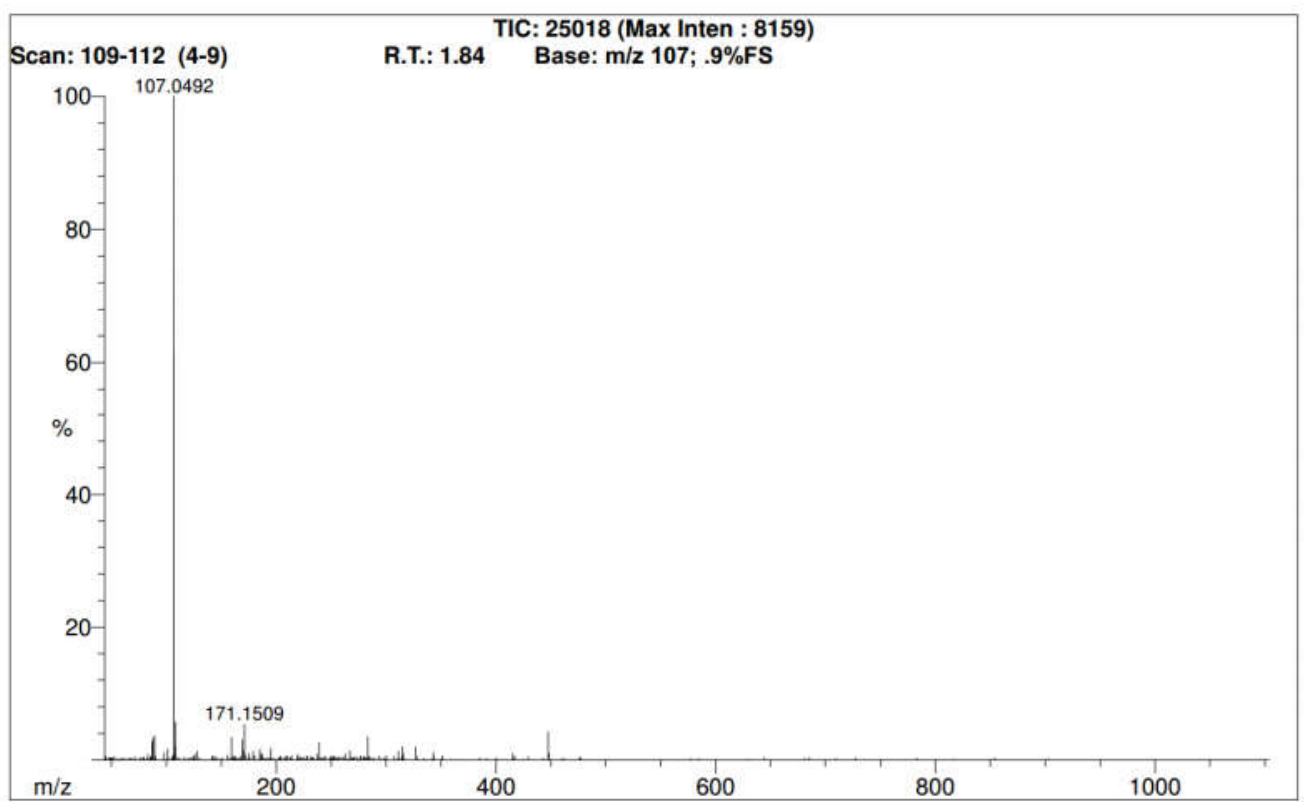

Figure 79 - Mass spectrum of benzaldehyde (trial \#2) at distance 2m, first detected at 1.84 $(1: 50)$ minutes. 
Table 18 - Summary of results for Benzaldehyde at all distances.

\begin{tabular}{|c|c|c|c|c|}
\hline \multicolumn{5}{|c|}{ Benzaldehyde at $0.5 \mathrm{~m}\left(400^{\circ} \mathrm{C}\right)$} \\
\hline Trial & Vial Open & Vial Closed & Detected & Time (s) \\
\hline$\# 1$ & 0:57 & 3:00 & 1:00 & 3 \\
\hline$\# 2$ & $1: 20$ & $3: 23$ & $1: 30$ & 10 \\
\hline$\# 3$ & $0: 49$ & $2: 52$ & $0: 58$ & 9 \\
\hline \multicolumn{5}{|c|}{ Benzaldehyde at $1 \mathrm{~m}\left(400^{\circ} \mathrm{C}\right)$} \\
\hline Trial & Vial Open & Vial Closed & Detected & Time (s) \\
\hline$\# 1$ & $0: 58$ & 3:03 & $1: 18$ & 20 \\
\hline$\# 2$ & 1:24 & 3:30 & 1:41 & 17 \\
\hline$\# \mathbf{3}$ & $0: 56$ & $2: 59$ & $1: 20$ & 24 \\
\hline \multicolumn{5}{|c|}{ Benzaldehyde at $1.5 \mathrm{~m}\left(400^{\circ} \mathrm{C}\right)$} \\
\hline Trial & Vial Open & Vial Closed & Detected & Time (s) \\
\hline$\# 1$ & 1:01 & 3:09 & 1:31 & 30 \\
\hline$\# 2$ & $1: 15$ & $3: 19$ & $1: 46$ & 31 \\
\hline \#3 & $1: 21$ & $3: 17$ & 1:51 & 30 \\
\hline \multicolumn{5}{|c|}{ Benzaldehyde at $2 \mathrm{~m}\left(400^{\circ} \mathrm{C}\right)$} \\
\hline Trial & Vial Open & Vial Closed & Detected & Time (s) \\
\hline$\# 1$ & 1:06 & 3:08 & $1: 42$ & 36 \\
\hline$\# 2$ & 1:06 & 3:08 & $1: 50$ & 44 \\
\hline \#3 & 1:07 & $3: 15$ & $1: 48$ & 41 \\
\hline \multicolumn{5}{|c|}{ Benzaldehyde at $3 \mathrm{~m}\left(400^{\circ} \mathrm{C}\right)$} \\
\hline Trial & Vial Open & Vial Closed & Detected & Time (s) \\
\hline$\# 1$ & $1: 13$ & $3: 17$ & $1: 57$ & 44 \\
\hline$\# 2$ & 1:16 & 3:19 & $1: 59$ & 43 \\
\hline$\# \mathbf{3}$ & 1:16 & $3: 19$ & 1:59 & 43 \\
\hline
\end{tabular}

The data from Table 18 was averaged at each distance and the standard deviation calculated. At $0.5 \mathrm{~m}$, it took $7.3 \pm 3.8$ seconds for benzaldehyde to be detected initially. At $1 \mathrm{~m}$, it took $20.3 \pm 3.5$ seconds. At $1.5 \mathrm{~m}$, it took $30.3 \pm 0.6$ seconds. At $2 \mathrm{~m}$, it took $40.3 \pm$ 4.0 seconds. Lastly, at $3 \mathrm{~m}$, it took $43.3 \pm 0.6$ seconds. These results were plotted and are shown in Figure 80. 


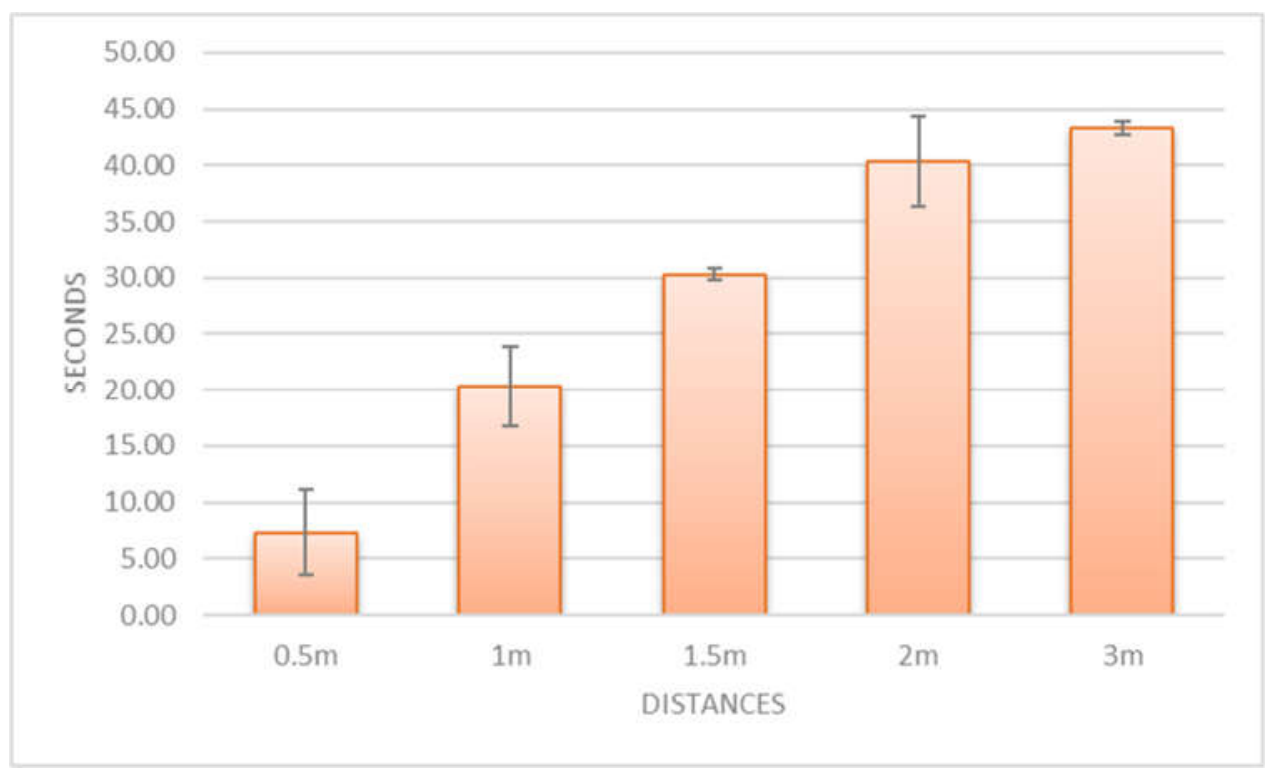

Figure 80 - Averaged results of task 2 study for benzaldehyde at the various distances.

\section{2-ethyl-1-hexanol}

For 2-ethyl-1-hexanol, there wasn't a single data set at any distance or trial that successfully detected the standard. Figure 81 shows the RIC where only $261 \mathrm{~m} / \mathrm{z}$ is detected, and as shown, there is no slow progression of detectable analytes present. The three peaks in the beginning are from PEG. Again, every run ends with a direct sampling of the standard to serve as a positive control. In all runs, the positive control was correctly detected and identified (Figure 82). Other examples are shown in Figure 83 and Figure 84 that show RIC's for 2-ethyl-1-hexanol at $2 \mathrm{~m}$ and $3 \mathrm{~m}$ respectively.

To ensure that 2-ethyl-1-hexanol does not require longer exposure time away from the DART source, it was tested at $0.5 \mathrm{~m}$ again while keeping the vial open for 10 minutes as opposed to the 2 minutes done for the other samples. Again, no detectable amount of 2ethyl-1-hexanol was identified except for the positive control. 


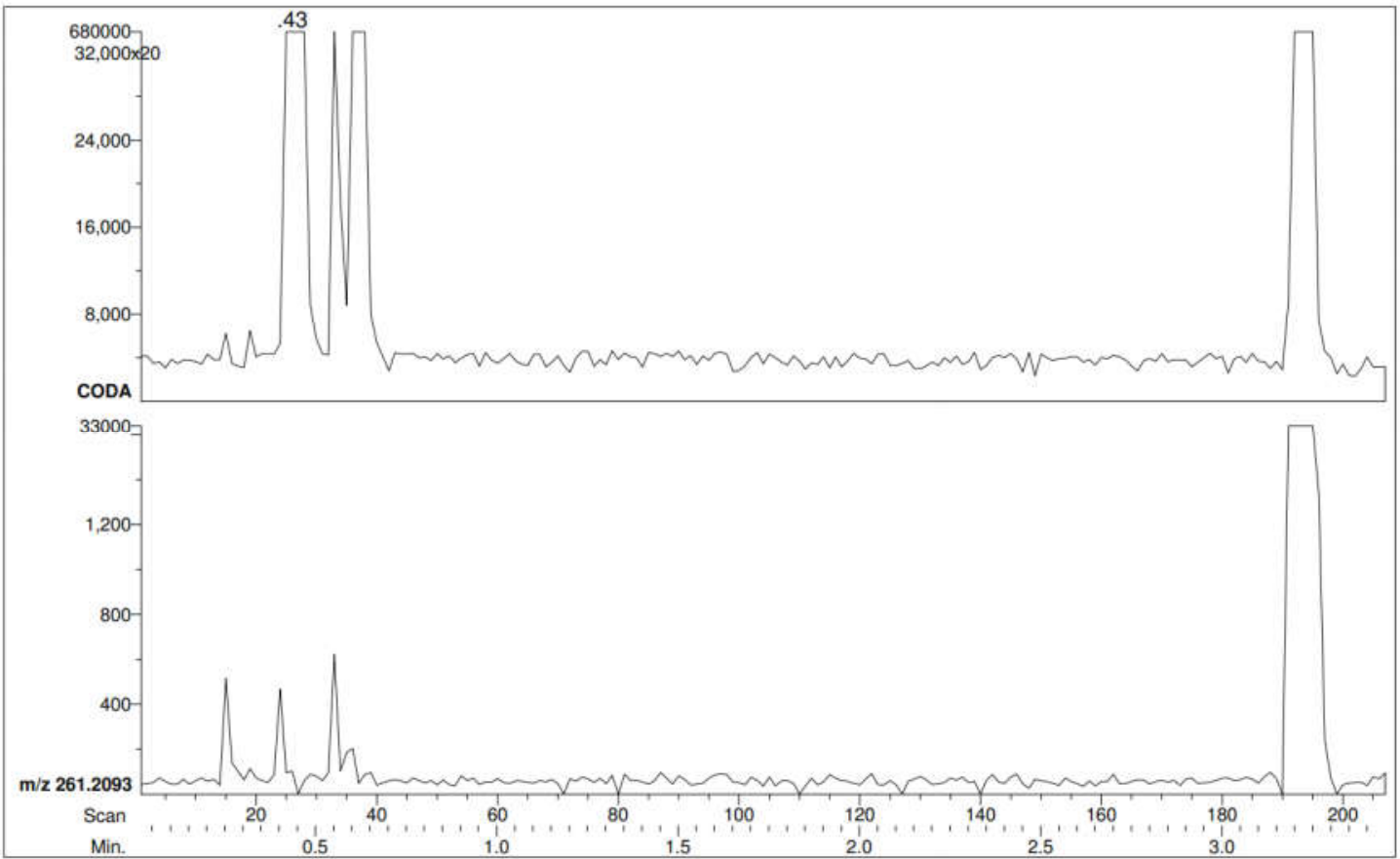

Figure 81 - RIC's of 2-ethyl-1-hexanol at a distance of $0.5 \mathrm{~m}$ (Trial \#3). Top: RIC after performing CODA (zoomed in). Bottom: RIC showing only peaks where $261 \mathrm{~m} / \mathrm{z}$ was detected. Cap opened at 58 seconds (0:58 min).

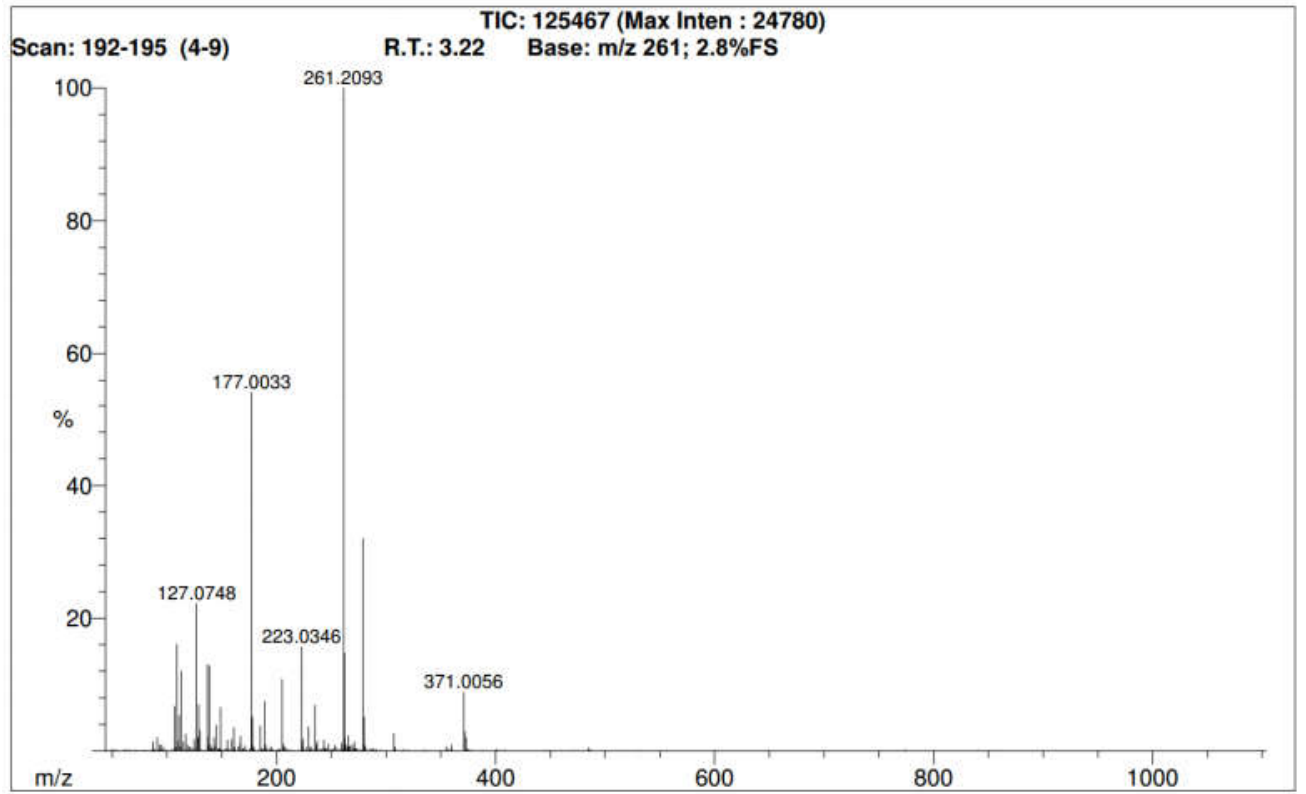

Figure 82 - Mass spectrum of 2-ethyl-1-hexanol positive control. 


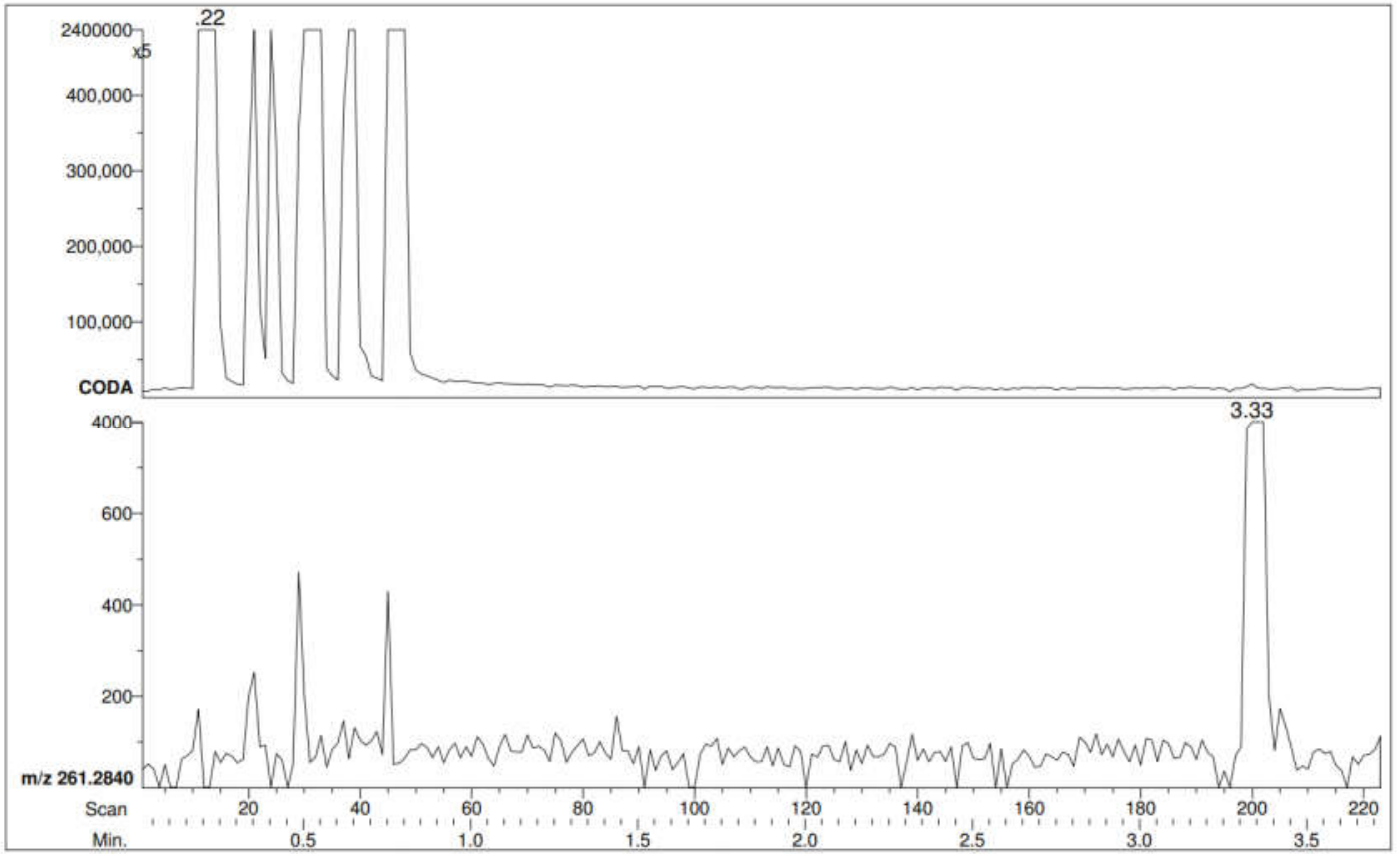

Figure 83 - RIC's of 2-ethyl-1-hexanol at a distance of $2 \mathrm{~m}$ (Trial \#1). Top: RIC after performing CODA (zoomed in). Bottom: RIC showing only peaks where $261 \mathrm{~m} / \mathrm{z}$ was detected. Cap opened at 1 minute and 5 seconds (1:05 min).

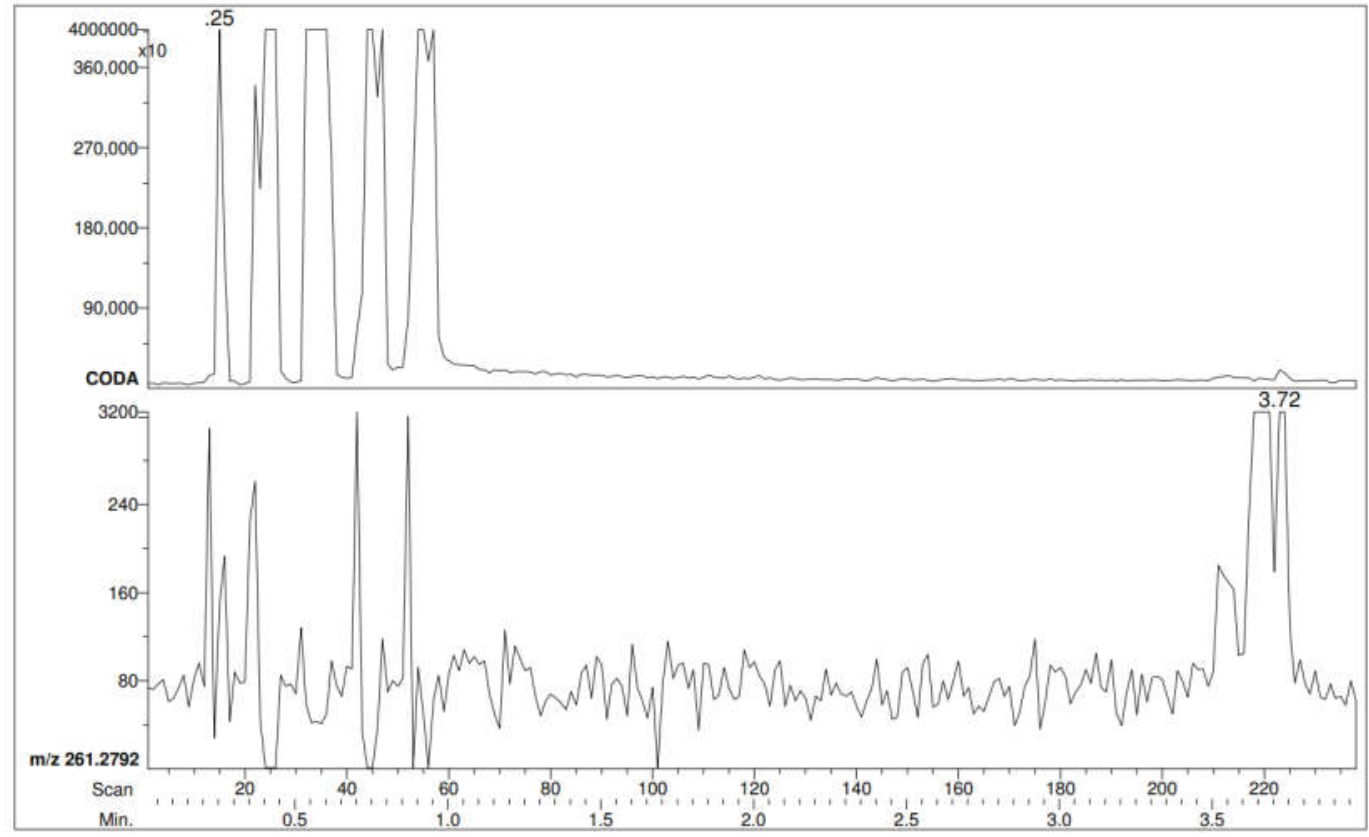

Figure 84 - RIC's of 2-ethyl-1-hexanol at a distance of 3m (Trial \#3). Top: RIC after performing CODA (zoomed in). Bottom: RIC showing only peaks where $261 \mathrm{~m} / \mathrm{z}$ was detected. Cap opened at 1 minute and 18 seconds (1:18 min). 
Table 19 - Summary of results for 2-ethyl-1-hexanol at all distances. (DND = Did not detect, N/A = Not applicable)

\begin{tabular}{|c|c|c|c|c|}
\hline \multicolumn{5}{|c|}{ 2-ethyl-1-hexanol at $0.5 \mathrm{~m}\left(400^{\circ} \mathrm{C}\right)$} \\
\hline Trial & Vial Open & Vial Closed & Detected & Time (s) \\
\hline$\# 1$ & $1: 09$ & $3: 12$ & DND & N/A \\
\hline$\# 2$ & 0:59 & 3:03 & DND & $\mathbf{N} / \mathbf{A}$ \\
\hline$\# \mathbf{3}$ & $0: 58$ & 3:04 & DND & $\mathbf{N} / \mathbf{A}$ \\
\hline \multicolumn{5}{|c|}{ 2-ethyl-1-hexanol at $1 \mathrm{~m}\left(400^{\circ} \mathrm{C}\right)$} \\
\hline Trial & Vial Open & Vial Closed & Detected & Time (s) \\
\hline$\# 1$ & $0: 51$ & $2: 54$ & DND & $\mathbf{N} / \mathbf{A}$ \\
\hline$\# 2$ & $1: 13$ & $3: 16$ & DND & $\mathbf{N} / \mathbf{A}$ \\
\hline$\# \mathbf{3}$ & $0: 59$ & $3: 02$ & DND & $\mathbf{N} / \mathbf{A}$ \\
\hline \multicolumn{5}{|c|}{ 2-ethyl-1-hexanol at $1.5 \mathrm{~m}\left(400^{\circ} \mathrm{C}\right)$} \\
\hline Trial & Vial Open & Vial Closed & Detected & Time (s) \\
\hline$\# 1$ & $0: 54$ & $2: 58$ & DND & N/A \\
\hline$\# 2$ & $0: 47$ & $2: 49$ & DND & $\mathbf{N} / \mathbf{A}$ \\
\hline$\# \mathbf{3}$ & $1: 14$ & $3: 25$ & DND & $\mathbf{N} / \mathbf{A}$ \\
\hline \multicolumn{5}{|c|}{ 2-ethyl-1-hexanol at $2 \mathrm{~m}\left(400^{\circ} \mathrm{C}\right)$} \\
\hline Trial & Vial Open & Vial Closed & Detected & Time (s) \\
\hline$\# 1$ & $1: 05$ & $3: 08$ & DND & N/A \\
\hline$\# 2$ & $1: 05$ & $3: 15$ & DND & N/A \\
\hline$\# 3$ & $1: 08$ & $3: 14$ & DND & $\mathbf{N} / \mathbf{A}$ \\
\hline \multicolumn{5}{|c|}{ 2-ethyl-1-hexanol at $3 \mathrm{~m}\left(400^{\circ} \mathrm{C}\right)$} \\
\hline Trial & Vial Open & Vial Closed & Detected & Time (s) \\
\hline$\# 1$ & $1: 14$ & $3: 18$ & DND & N/A \\
\hline$\# 2$ & $1: 21$ & $3: 24$ & DND & $\mathbf{N} / \mathbf{A}$ \\
\hline$\# 3$ & $1: 18$ & $3: 21$ & DND & $\mathbf{N} / \mathbf{A}$ \\
\hline
\end{tabular}

This data suggests that 2-ethyl-1-hexanol as a VOC does not travel in the air and spread via advection in a similar fashion to methyl benzoate and benzaldehyde. Instead, it seems that these VOC's volatilize and stay localized around the area in which it was introduced. 


\section{Diethyl Phthalate}

Diethyl phthalate in this experiment has rather unique results. The data for all trials at each distance is shown in Table 20. While good results were obtained at distances $0.5 \mathrm{~m}$, $1 \mathrm{~m}$ and $1.5 \mathrm{~m}$, diethyl phthalate was not detected during any trial at distances of $2 \mathrm{~m}$ and $3 \mathrm{~m}$. Also, unlike methyl benzoate and benzaldehyde, the RIC's for these diethyl phthalate tests do not show a slow progression of the standard as time goes on (Figure 85 and Figure 86). In fact, in many instances for diethyl phthalate, the sample was detected in areas that are not obvious in their visibility in the RIC. Interestingly, Figure 85 shows instances of higher intensity peaks (at $\sim 1.2$ min and $\sim 2$ min mark) which could indicate the presence of eddies (as discussed in section 2.3.7 and illustrated in Figure 16) from within the odor plume.

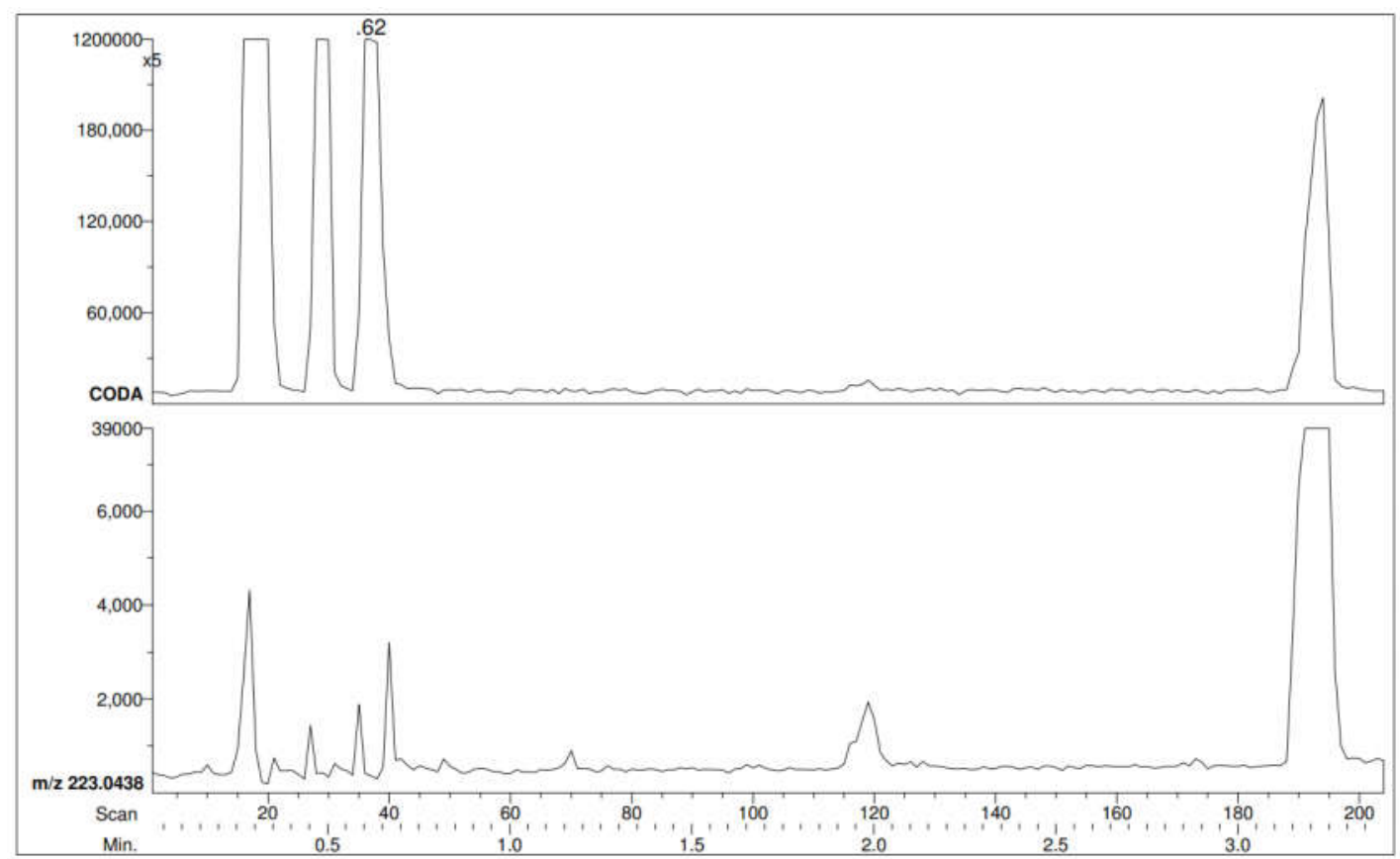

Figure 85 - RIC's of diethyl phthalate at a distance of $0.5 \mathrm{~m}$ (Trial \#2). Top: RIC after performing CODA (zoomed in). Bottom: RIC showing only peaks where $223 \mathrm{~m} / \mathrm{z}$ was detected. Cap opened at 57 seconds (0:57 min). 


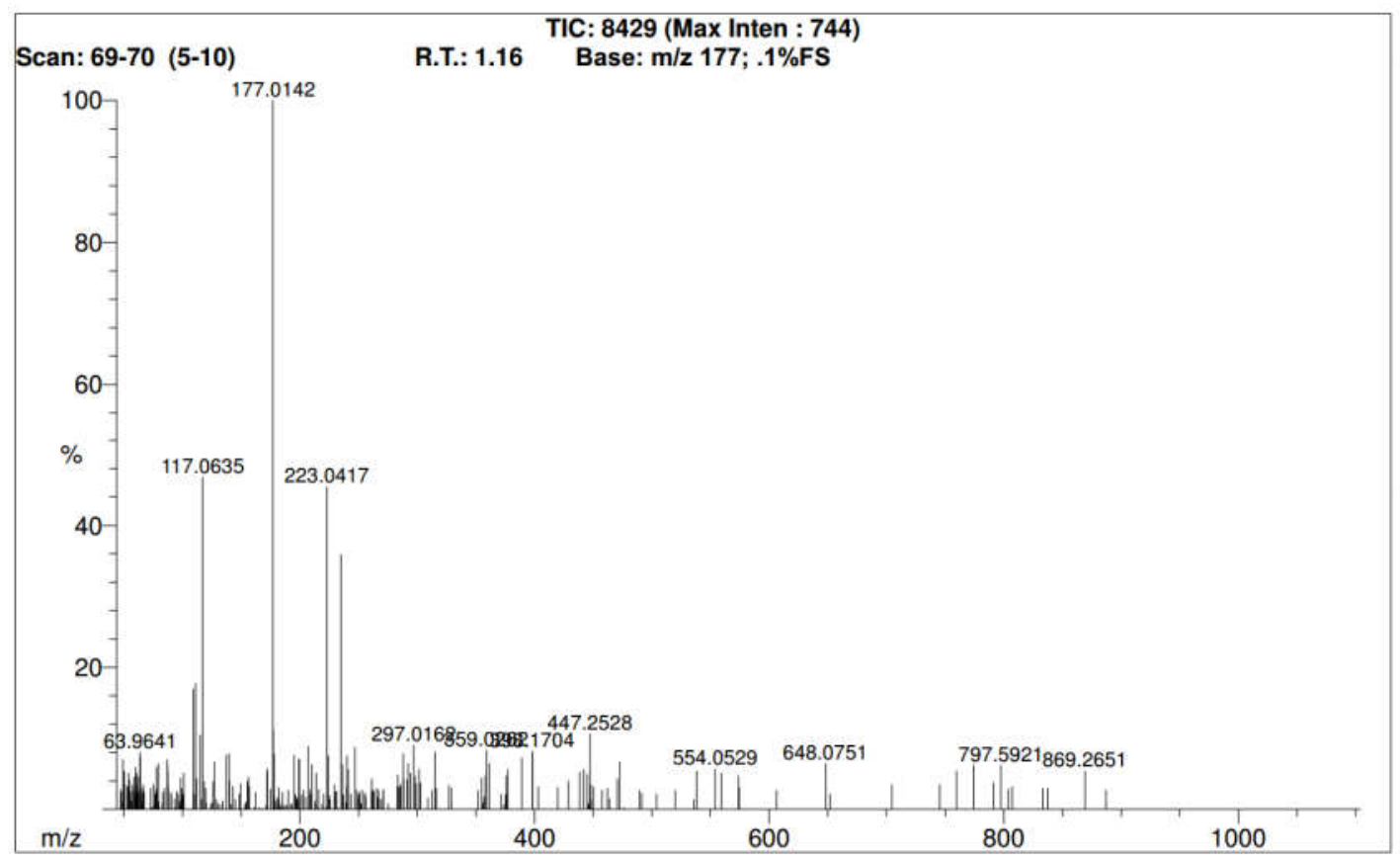

Figure 86 - Mass spectrum of diethyl phthalate (trial \#2) at distance $0.5 \mathrm{tm}$, first detected at $1.16(1: 09)$ minutes.

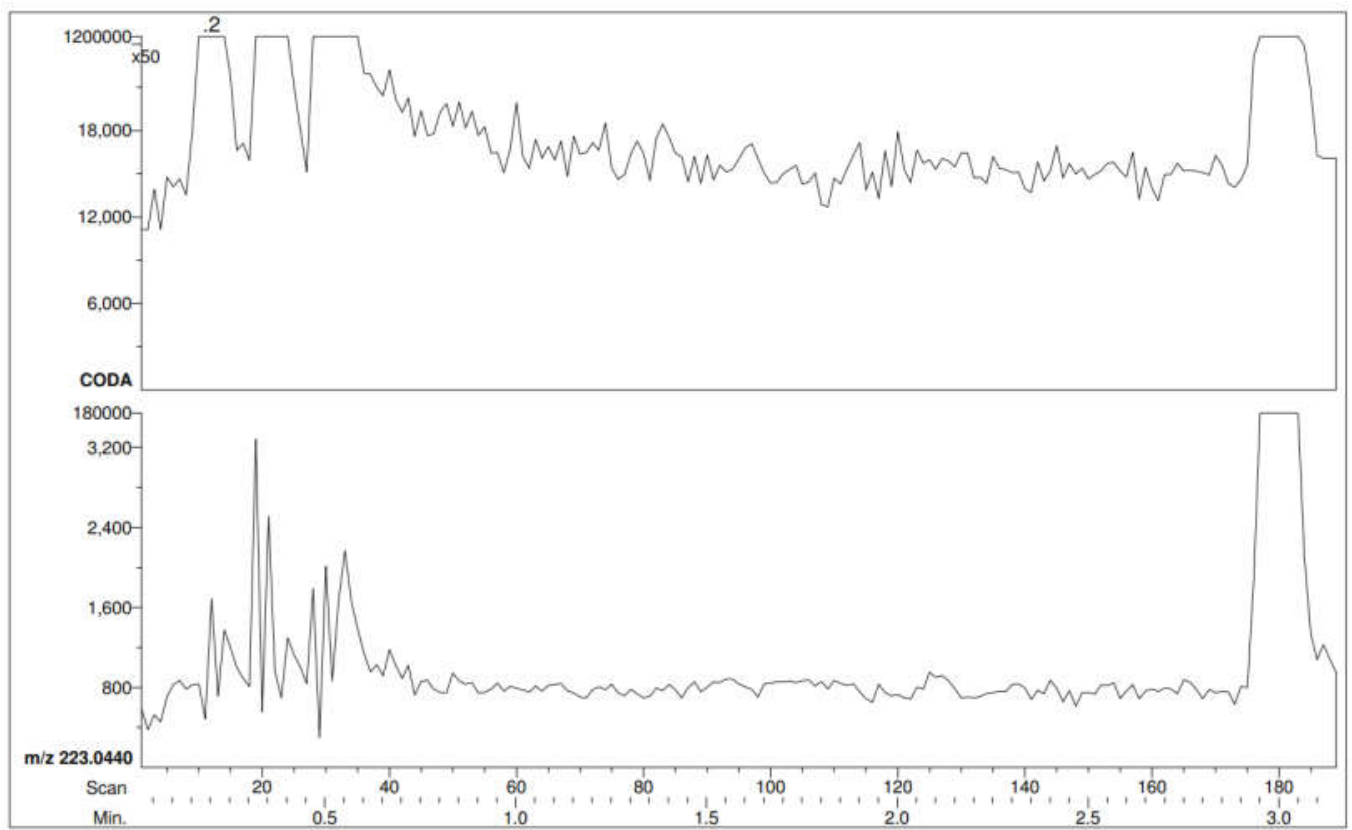

Figure 87 - RIC's of diethyl phthalate at a distance of 1.5m (Trial \#2). Top: RIC after performing CODA (zoomed in). Bottom: RIC showing only peaks where $223 \mathrm{~m} / \mathrm{z}$ was detected. Cap opened at 48 seconds $(0: 48 \mathrm{~min})$. 


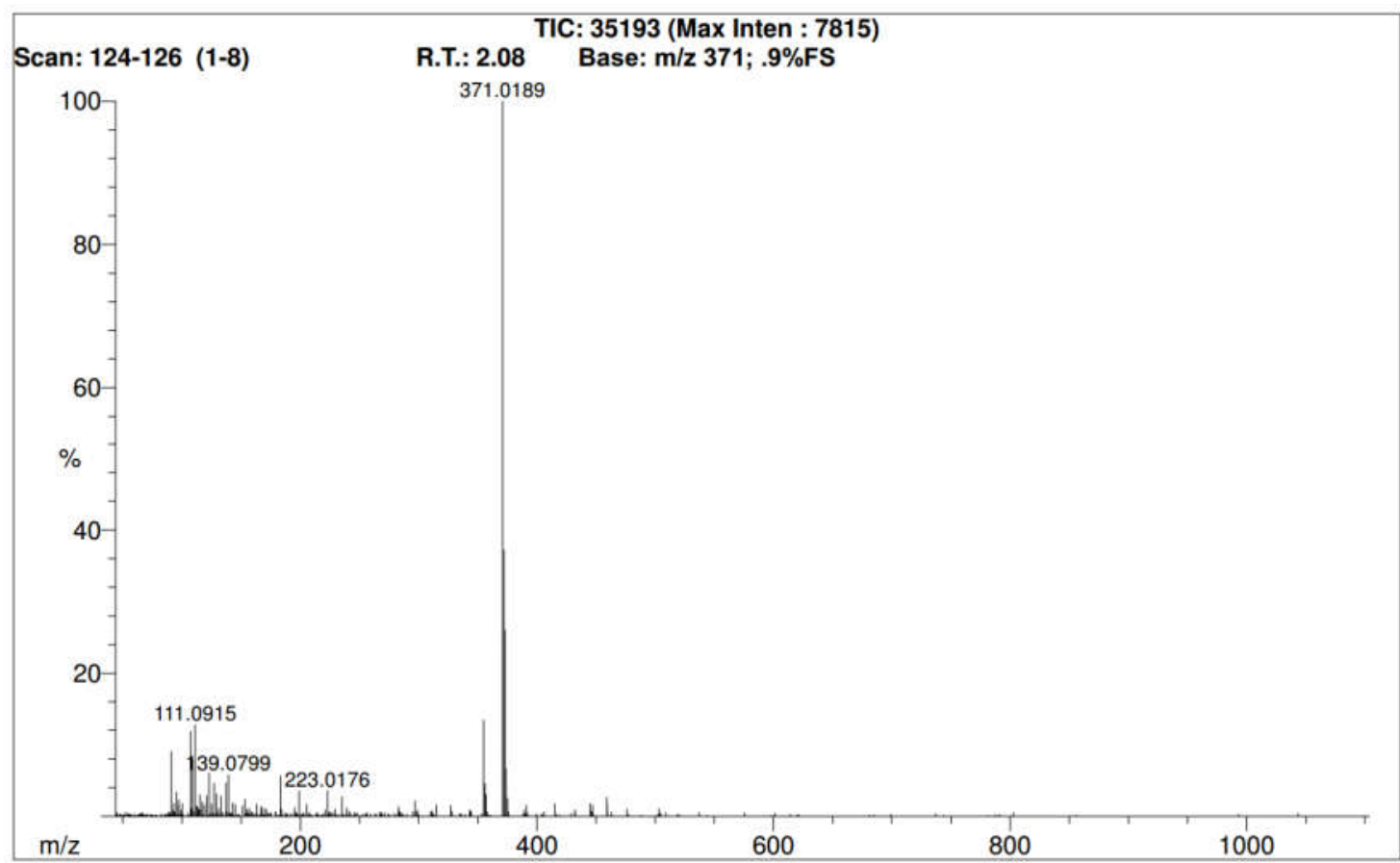

Figure 88 - Mass spectrum of diethyl phthalate (trial \#2) at distance 1.5tm, first detected at $2.08(2: 05)$ minutes. Intensity for $223 \mathrm{~m} / \mathrm{z}$ was above $5 \%$ relative intensity.

The data suggests that diethyl phthalate is not as volatile in its movement as methyl benzoate and benzaldehyde, yet more volatile than 2-ethyl-1-hexanol. As was done with 2ethyl-1-hexanol, the exposure time was extended to 10 minutes at distances of 2 and 3 meters to no avail. The advection process that moves the odor plume for diethyl phthalate seems to dilute in its concentration to levels not detectable by the instrument somewhere between $1.5 \mathrm{~m}-2 \mathrm{~m}$.

For purposes of uniformity, Table 20 shows the data for two-minute exposure times. For diethyl phthalate, the average time it took for the DART to detect the sample at a distance of $0.5 \mathrm{~m}$ was $7.3 \pm 2.6$ seconds. At a distance of $1 \mathrm{~m}$, it took $20.3 \pm 2.0$ seconds and at $1.5 \mathrm{~m}$, it took $30 \pm 4.0$ seconds on average. Again, diethyl phthalate was not detected at distances of $2 \mathrm{~m}$ and $3 \mathrm{~m}$. 
Table 20 - Summary of results for diethyl phthalate at all distances. (DND $=$ Did not detect, N/A = Not applicable)

\begin{tabular}{|c|c|c|c|c|}
\hline \multicolumn{5}{|c|}{ Diethyl Phthalate at $0.5 \mathrm{~m}\left(400^{\circ} \mathrm{C}\right)$} \\
\hline Trial & Vial Open & Vial Closed & Detected & Time (s) \\
\hline$\# 1$ & $0: 45$ & $2: 47$ & $0: 52$ & 7 \\
\hline$\# 2$ & 0:57 & 3:04 & 1:09 & 12 \\
\hline$\# 3$ & 1:16 & $3: 19$ & $1: 24$ & 8 \\
\hline \multicolumn{5}{|c|}{ Diethyl Phthalate at $1 \mathrm{~m}\left(400^{\circ} \mathrm{C}\right)$} \\
\hline Trial & Vial Open & Vial Closed & Detected & Time (s) \\
\hline$\# 1$ & $1: 11$ & $3: 14$ & $1: 48$ & 37 \\
\hline$\# 2$ & 1:01 & 3:04 & $1: 40$ & 39 \\
\hline$\# \mathbf{3}$ & 1:07 & $3: 10$ & $1: 48$ & 41 \\
\hline \multicolumn{5}{|c|}{ Diethyl Phthalate at $1.5 \mathrm{~m}\left(400^{\circ} \mathrm{C}\right)$} \\
\hline Trial & Vial Open & Vial Closed & Detected & Time (s) \\
\hline$\# 1$ & $1: 12$ & $3: 14$ & $2: 22$ & 70 \\
\hline$\# 2$ & $0: 48$ & $2: 50$ & 2:05 & 78 \\
\hline$\# \mathbf{3}$ & $1: 13$ & $3: 35$ & $2: 28$ & 74 \\
\hline \multicolumn{5}{|c|}{ Diethyl Phthalate at $2 \mathrm{~m}\left(400^{\circ} \mathrm{C}\right)$} \\
\hline Trial & Vial Open & Vial Closed & Detected & Time (s) \\
\hline$\# 1$ & $1: 18$ & $3: 21$ & DND & N/A \\
\hline$\# 2$ & 1:18 & $3: 10$ & DND & $\mathbf{N} / \mathbf{A}$ \\
\hline$\# \mathbf{3}$ & $1: 14$ & $3: 20$ & DND & $\mathbf{N} / \mathbf{A}$ \\
\hline \multicolumn{5}{|c|}{ Diethyl Phthalate at $3 \mathrm{~m}\left(400^{\circ} \mathrm{C}\right)$} \\
\hline Trial & Vial Open & Vial Closed & Detected & Time (s) \\
\hline$\# 1$ & $1: 12$ & $3: 16$ & DND & N/A \\
\hline$\# 2$ & 1:11 & $3: 14$ & DND & N/A \\
\hline$\# \mathbf{3}$ & $1: 16$ & 3:19 & DND & N/A \\
\hline
\end{tabular}

Figure 89 shows the results of the data in a visual chart. Unlike methyl benzoate and benzaldehyde, diethyl phthalate's results show a clear distinction in time at the various distances. With methyl benzoate, the results at $1 \mathrm{~m}$ and $1.5 \mathrm{~m}$ are virtually indistinguishable, and for benzaldehyde, the results at $2 \mathrm{~m}$ and $3 \mathrm{~m}$ are practically identical. 


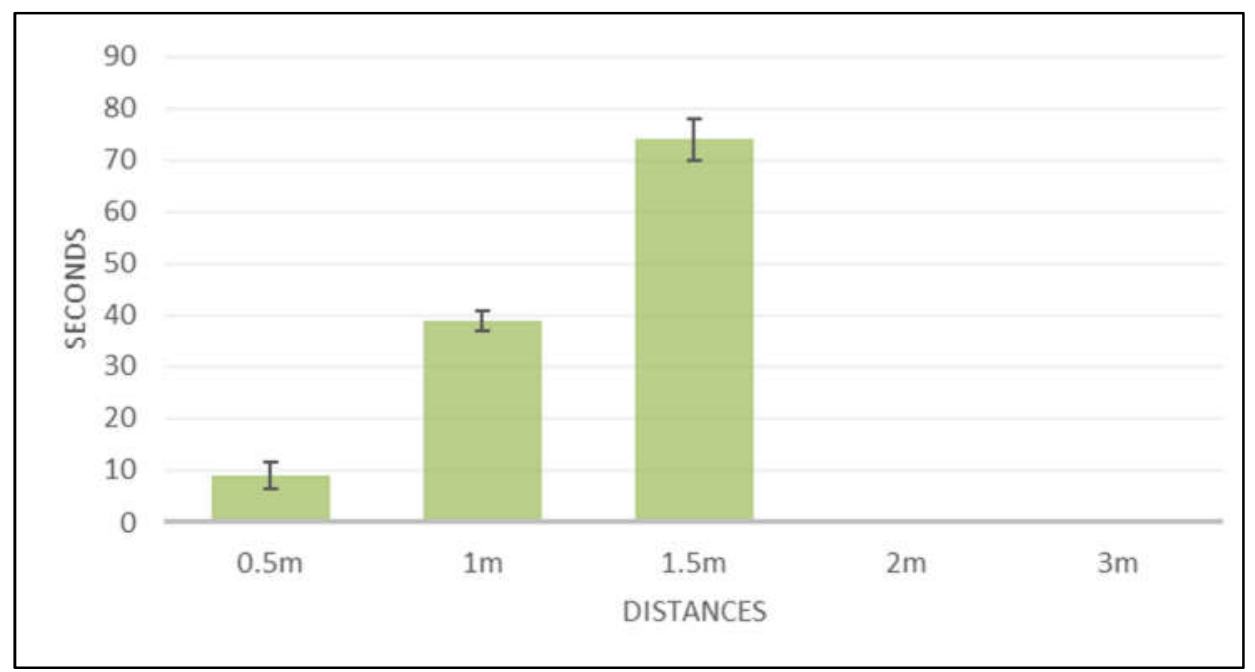

Figure 89 - Averaged results of task 2 study for diethyl phthalate at the various distances. No results obtained at distances $2 \mathrm{~m}$ and $3 \mathrm{~m}$.

In conclusion, the data suggests that the odors emitting from illicit substances do travel in relatively short periods of time, and that could explain why canines sometimes alert at objects that do not contain an illicit substance. This could be one of the several reasons why there are unconfirmed alerts during canine trials. The summary of the data for the four chemical standards are shown in Table 21 and Figure 90.

Table 21 - Summary of averaged times for all four chemical standards.

\begin{tabular}{|c|c|c|c|c|c|}
\hline & $0.5 \mathrm{~m}$ & $1 \mathrm{~m}$ & $1.5 \mathrm{~m}$ & $2 \mathrm{~m}$ & $3 \mathrm{~m}$ \\
\hline Sample & \multicolumn{4}{|c|}{ Time needed for initial detection (s) } \\
\hline Methyl Benzoate & $\mathbf{6 . 0} \pm 1.7$ & $\mathbf{2 0 . 0} \pm 4.4$ & $\mathbf{2 3 . 3} \pm 1.2$ & $\mathbf{3 1 . 0} \pm \mathbf{2 . 0}$ & $\mathbf{3 9 . 3} \pm \mathbf{3 . 2}$ \\
\hline Benzaldehyde & $7.3 \pm 3.8$ & $\mathbf{2 0 . 3} \pm 3.5$ & $\mathbf{3 0 . 3} \pm \mathbf{0 . 6}$ & $\mathbf{4 0 . 3} \pm 4.0$ & $\mathbf{4 3 . 3} \pm \mathbf{0 . 6}$ \\
\hline 2-ethyl-1-hexanol & DND & DND & DND & DND & DND \\
\hline Diethyl Phthalate & $\mathbf{9 . 0} \pm \mathbf{2 . 6}$ & $\mathbf{3 9 . 0} \pm \mathbf{2 . 0}$ & $\mathbf{7 4 . 0} \pm 4.0$ & DND & DND \\
\hline
\end{tabular}




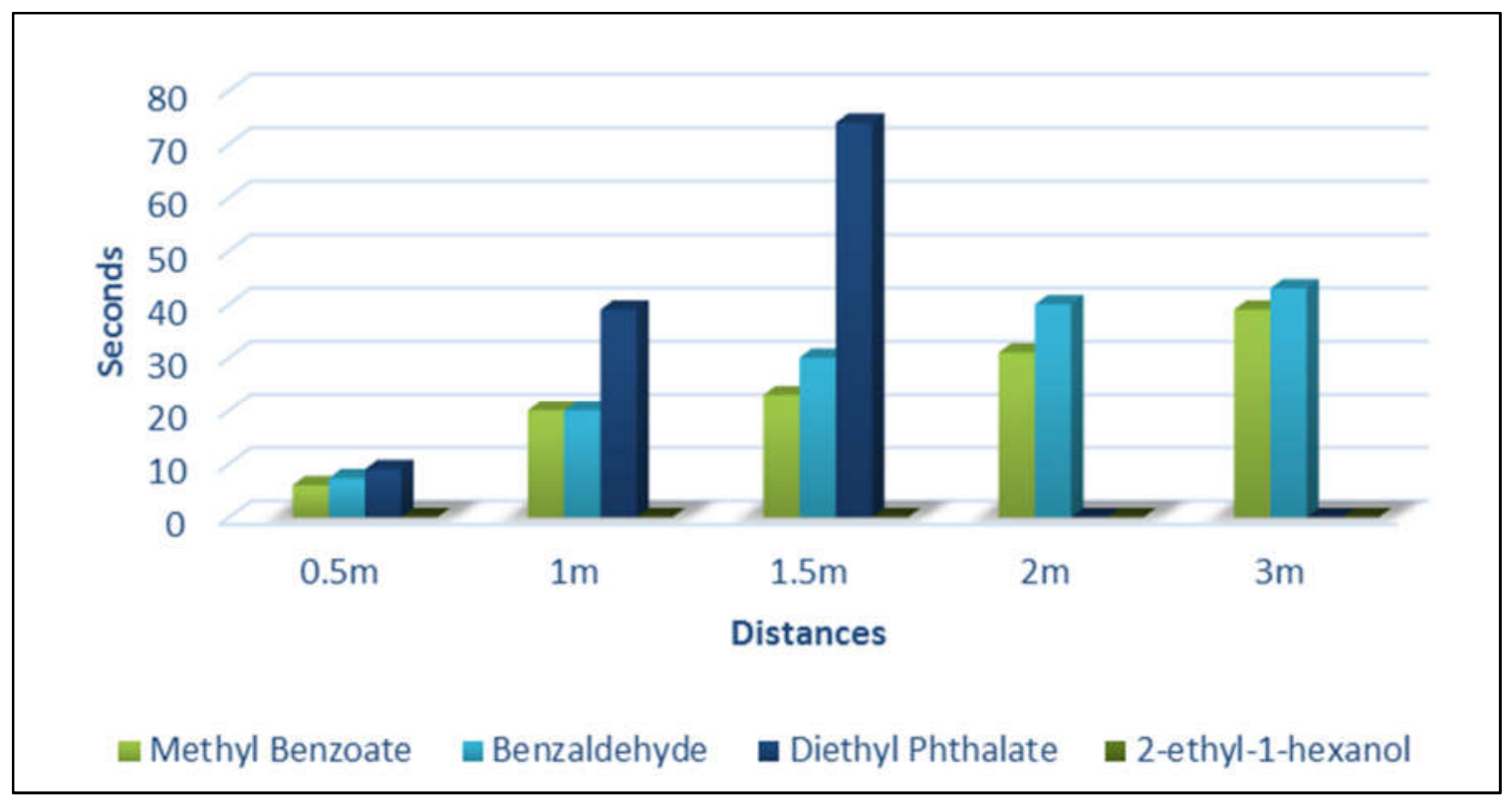

Figure 90 - Graph comparing the averaged results for the different standards at the different distances

\subsection{Task 3: Residual Odor Study}

While Task 2 attempted to study the amount of time it took for odorants to be detected at various distances, Task 3 attempts to answer the question about how long it takes for these same odors to persist on substrates typically encountered at a crime scene. For this task, wooden toothpicks, glass capillary tubes and metal paper clips were used as the substrates.

Part $A$

Originally, this task began with the use of a large $(62.5 \mathrm{~cm} \mathrm{~L} \mathrm{x} 47.6 \mathrm{~cm} \mathrm{~W} \mathrm{x} 17.5$ $\mathrm{cm} \mathrm{H})$ Sterilite ${ }^{\circledR}$ plastic container with lid. The substrates were tested in triplicate, meaning there were 3 pieces of each substrate for each exposure time. The exposure times were 10s, 30s, 1min, $2 \mathrm{~min}, 5 \mathrm{~min}, 10 \mathrm{~min}, 15 \mathrm{~min}, 30 \mathrm{~min}$ and 1 hour. As soon as the chemical standards are placed in the petri dish (placed in the middle of the container) and the lid is 
closed, the timer starts. After the appropriate exposure times have elapsed, the lid is removed and the substrates are sampled on the DART immediately. Seeing that the exposure times are rather short in time, only one substrate was sampled at a time. Care was taken to have all samples placed in areas of similar location relative to the petri dish.

Unfortunately, none of the standards were detected on any of the substrates at any of the exposure times (Table 22). These results, or lack thereof, could be a result of low exposure times overall, or due to the distances the samples are placed away from the petri dish. The lack of tangible results led to exploring this Task using a different approach for Part B.

Table 22 - Results for Task 3, Part A using a large plastic container. (DND = Did not detect)

\begin{tabular}{|c|c|c|c|c|c|}
\hline & Sample & $\begin{array}{c}\text { Methyl } \\
\text { Benzoate }\end{array}$ & Benzaldehyde & $\begin{array}{c}\text { 2-ethyl-1- } \\
\text { hexanol }\end{array}$ & $\begin{array}{c}\text { Diethyl } \\
\text { Phthalate }\end{array}$ \\
\hline \multirow{9}{*}{$\begin{array}{c}\text { Exposure } \\
\text { Time }\end{array}$} & $10 \mathrm{~s}$ & DND & DND & DND & DND \\
\hline & $30 \mathrm{~s}$ & DND & DND & DND & DND \\
\hline & $1 \mathrm{~min}$ & DND & DND & DND & DND \\
\hline & $2 \min$ & DND & DND & DND & DND \\
\hline & $5 \mathrm{~min}$ & DND & DND & DND & DND \\
\hline & $10 \mathrm{~min}$ & DND & DND & DND & DND \\
\hline & $15 \mathrm{~min}$ & DND & DND & DND & DND \\
\hline & $30 \mathrm{~min}$ & DND & DND & DND & DND \\
\hline & 1 hour & DND & DND & DND & DND \\
\hline
\end{tabular}

Part B

For Part B, the approach was to first allow the volatiles to saturate the area, by allowing it to be exposed over-night, followed by the placement of the substrate samples in the container for the appropriate amount of exposure time. The theory is that the odors would most likely persist after the volatiles have already heavily saturated the headspace 
of the container. For better control of this experiment, smaller plastic containers $(\mathrm{Glad} \AA$ $17.5 \mathrm{~cm} \times 17.3 \mathrm{~cm} \times 12.2 \mathrm{~cm}$ ) were used instead. Again, due to the relatively short amount of exposure time for some of these tests, one substrate was tested at a time.

This experiment did not produce the results expected, and in fact, similarly to Part A, none of the chemical standards were detected on any of the substrates for any of the exposure times. While these results could have been expected for the lower exposure times, it was surprising to see that it didn't produce any results at the 30 minute or 1 hour exposure times. The worry at this point was to try and determine if it was even possible to have these odors persist at any exposure time, or determine if the issue was an experimental flaw or potentially a result of human error.

From the literature, it was shown that cocaine can get detected on currency (using the DART) as a result of the money circulating with bank notes used by drug users[92]. However, the mass spectrum does not show a peak at $\mathrm{m} / \mathrm{z} 137$ that would indicate the presence of methyl benzoate. Thus, the theory then becomes that this may be a limitation of the instrument. However, methyl benzoate would only be present on the dollar bill if it were in recent contact, or close proximity, to cocaine[93]. In an effort to determine whether or not the goals of this experiment are limited as a result of the instrument, a simple experiment of exposing a dollar bill to methyl benzoate for 24 hours was done.

Since the interest is only whether methyl benzoate could be detected or not, the dollar bills were rinsed with ethanol and dried prior to its exposure to methyl benzoate. To increase the surface area of the bill, the bill was taped in the middle to the bottom of a mason jar lid (Figure 91 - Picture of the mason jar with the dollar bill exposed to methyl 
benzoate, placed in a petri dish at the bottom of the jar.). The result was that methyl benzoate was detected in abundance on the dollar bills, proving that the issues are not related to the instrument, but rather in the experimental design or implementation.

Therefore, this task was redirected in its approach to first determine if these chemicals can be detected on the three substrates chosen at longer exposure times, and second, to see if the intensity of the peaks (if present) can be correlated to concentration or exposure times.

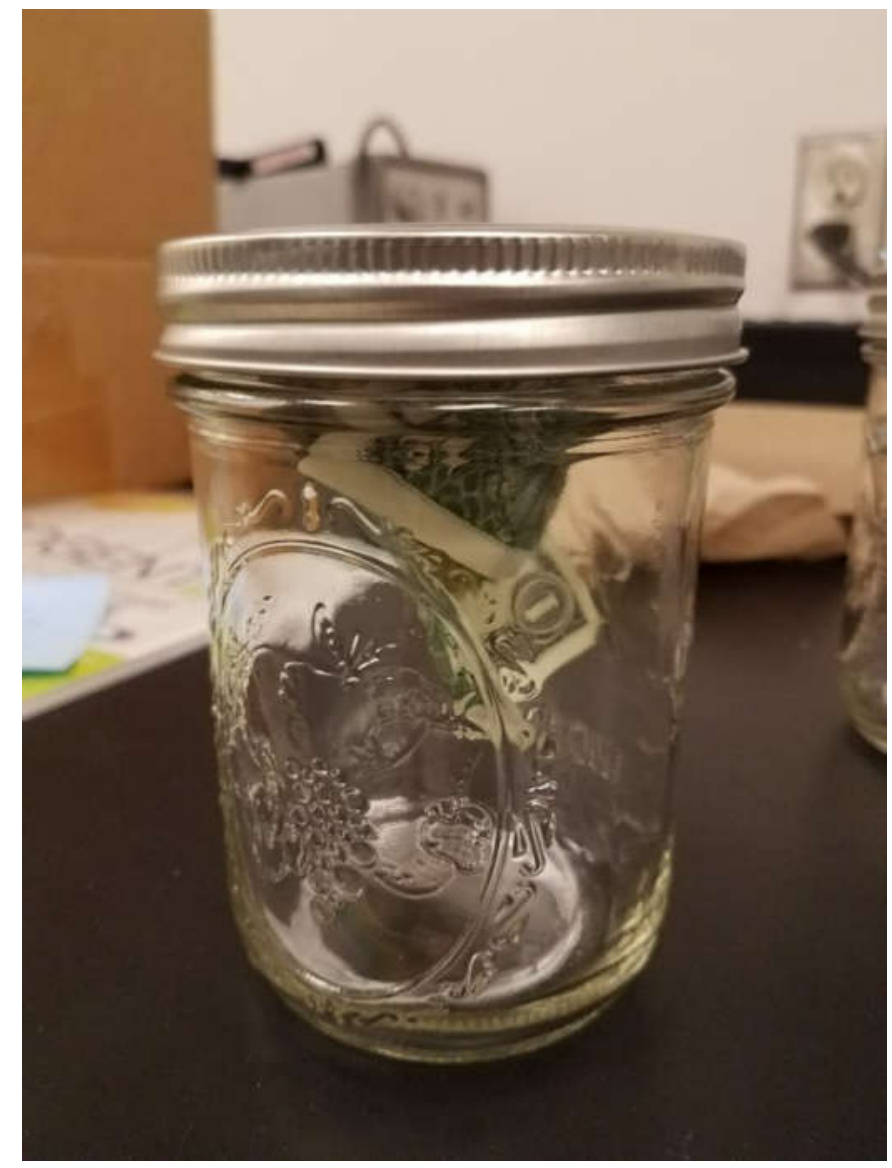

Figure 91 - Picture of the mason jar with the dollar bill exposed to methyl benzoate, placed in a petri dish at the bottom of the jar. 
Part C

The exposure times chosen for this portion of Task 3 were 2 hour intervals between 2 hours to 24 hours (for a total of 12 different exposure times). For each exposure time, four small Glad ${ }^{\circledR}$ plastic containers were used, containing 3 samples of each substrate (total 9 samples) in each box. The four boxes are for the four different chemical standards. A detailed explanation of this experiment is shown under methods in section 3.3.3.3.

A sample RIC for this test is shown in Figure 92. The sequence of sampling for this experiment at each exposure time is as follows:

\section{PEG $\times 3$}

2. Toothpicks exposed to methyl benzoate $\mathrm{x} 3$

3. Glass capillary tubes exposed to methyl benzoate $\mathrm{x} 3$

4. Metal clips exposed to methyl benzoate $\mathrm{x} 3$

5. Headspace of methyl benzoate standard (positive control)

6. Toothpicks exposed to benzaldehyde x 3

7. Glass capillary tubes exposed to benzaldehyde x 3

8. Metal clips exposed to benzaldehyde x 3

9. Headspace of benzaldehyde (positive control)

10. Toothpicks exposed to 2-ethyl-1-hexanol x 3

11. Glass capillary tubes exposed to 2-ethyl-1-hexanol x 3

12. Metal clips exposed to 2-ethyl-1-hexanol x 3

13. Headspace of 2-ethyl-1-hexanol (positive control)

14. Toothpicks exposed to diethyl phthalate $\mathrm{x} 3$ 
15. Glass capillary tubes exposed to diethyl phthalate $\mathrm{x} 3$

16. Metal clips exposed to diethyl phthalate $\mathrm{x} 3$

17. Headspace of diethyl phthalate (positive control)

After a two-hour exposure time, all 4 chemical standards were detected on the wooden toothpicks, but only benzaldehyde and diethyl phthalate were detected on the glass capillary tubes. None of the standards were detected on the metal clips. So far, these results indicate that the issues experienced in prior experiments may very well be down to low exposure times. The RIC's for all 12 exposure times are shown in Figure 92-Figure 103. 


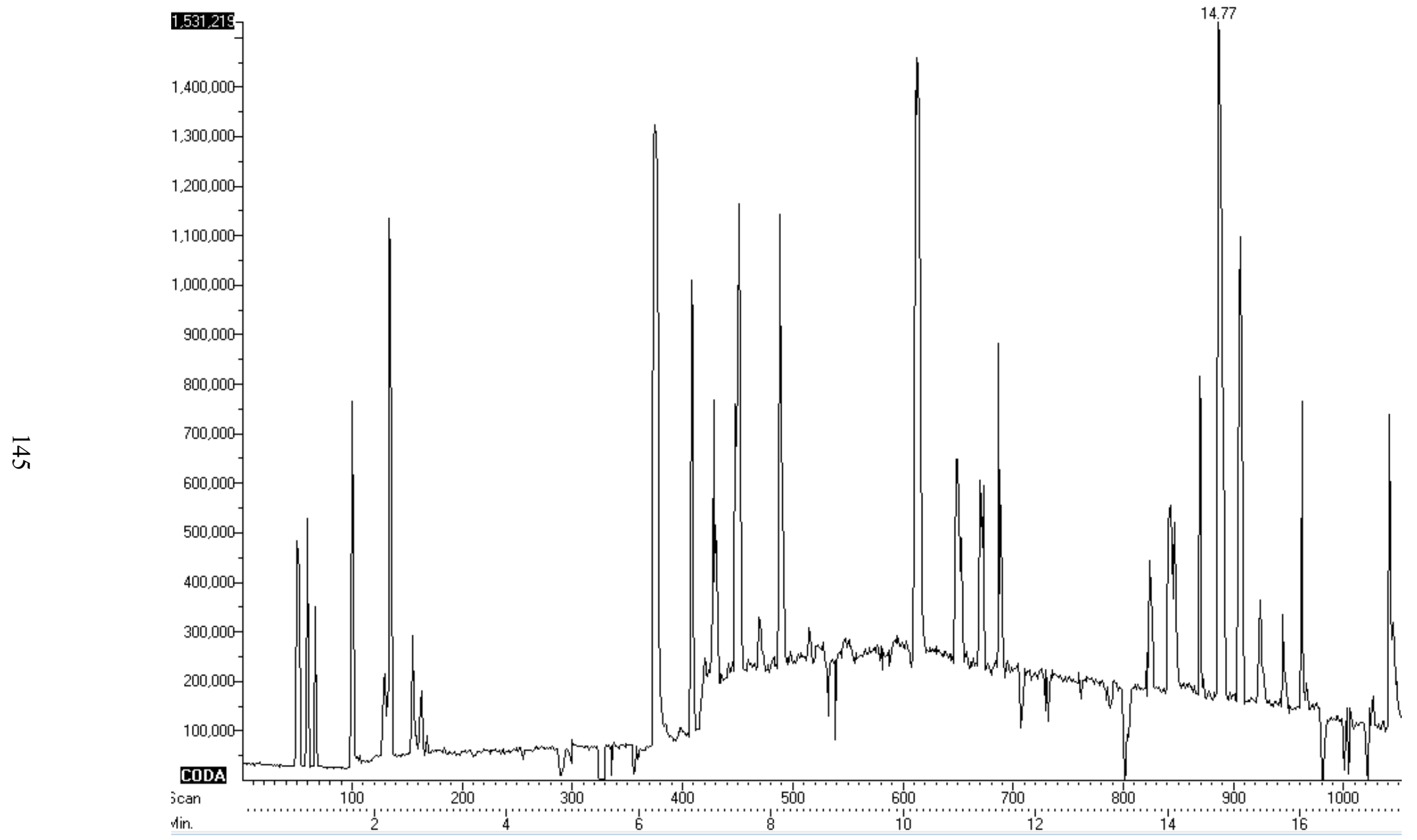

Figure 92 - RIC 2 hours 


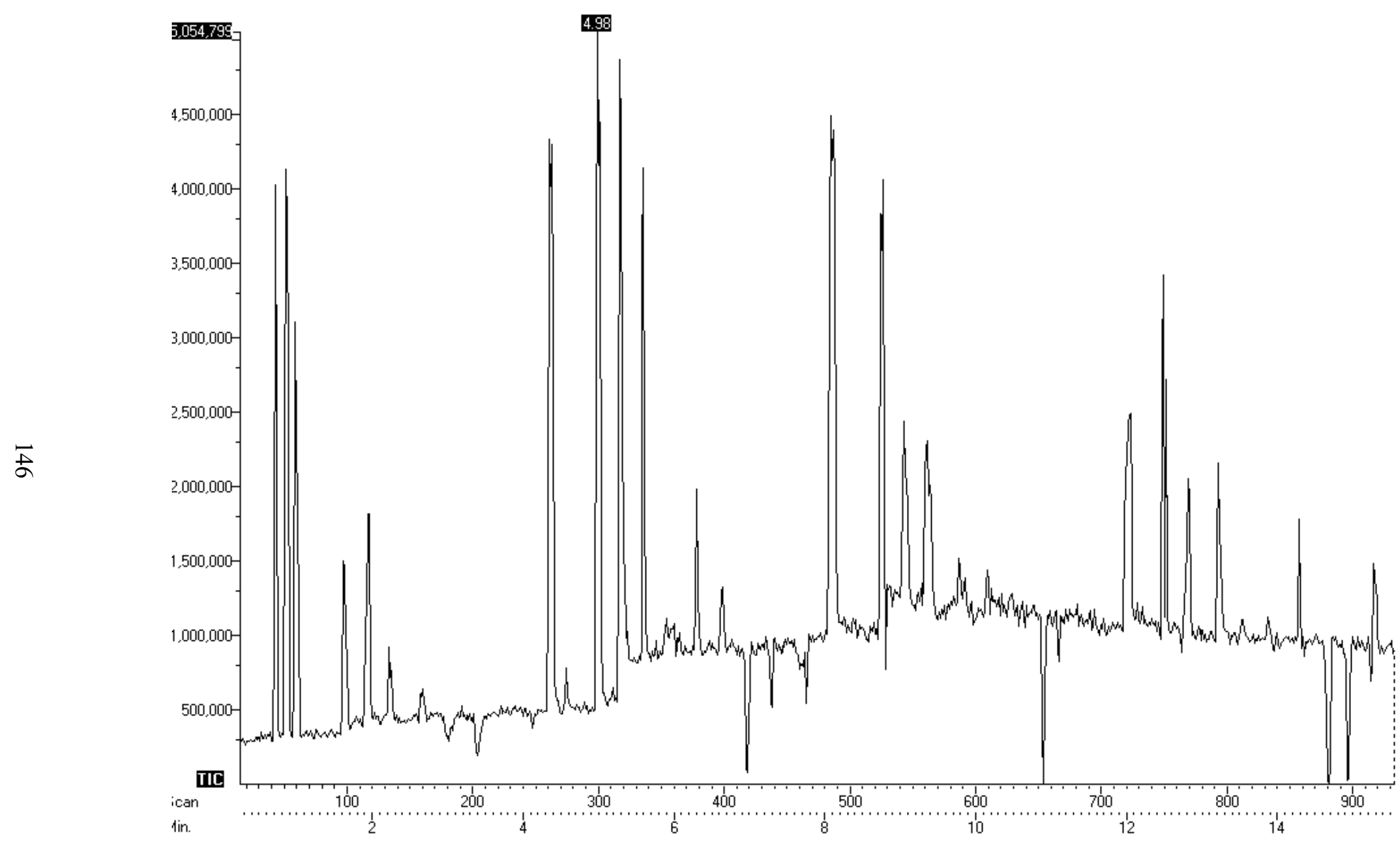

Figure 93 - RIC 4 hours 


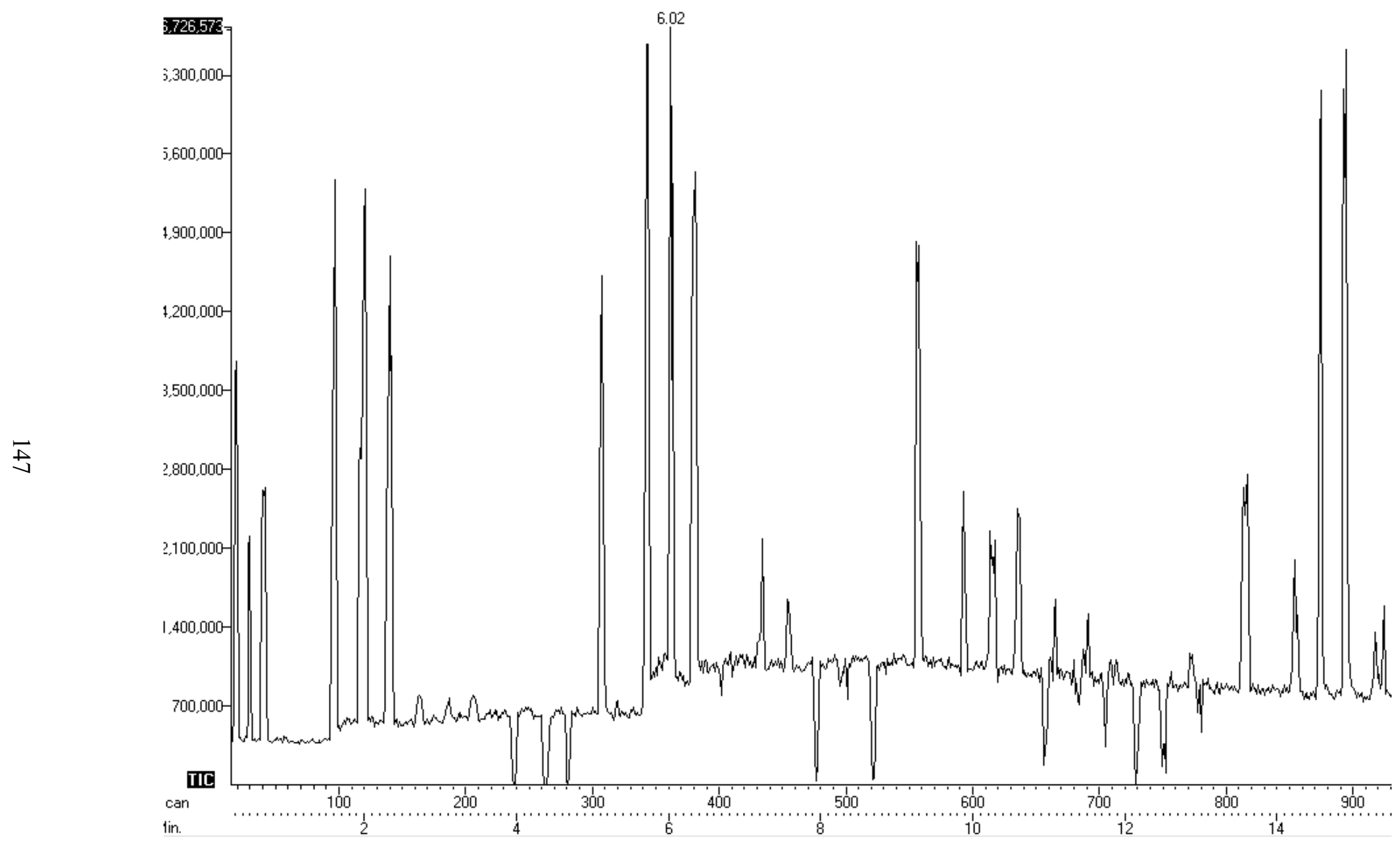

Figure 94 - RIC 6 hours 


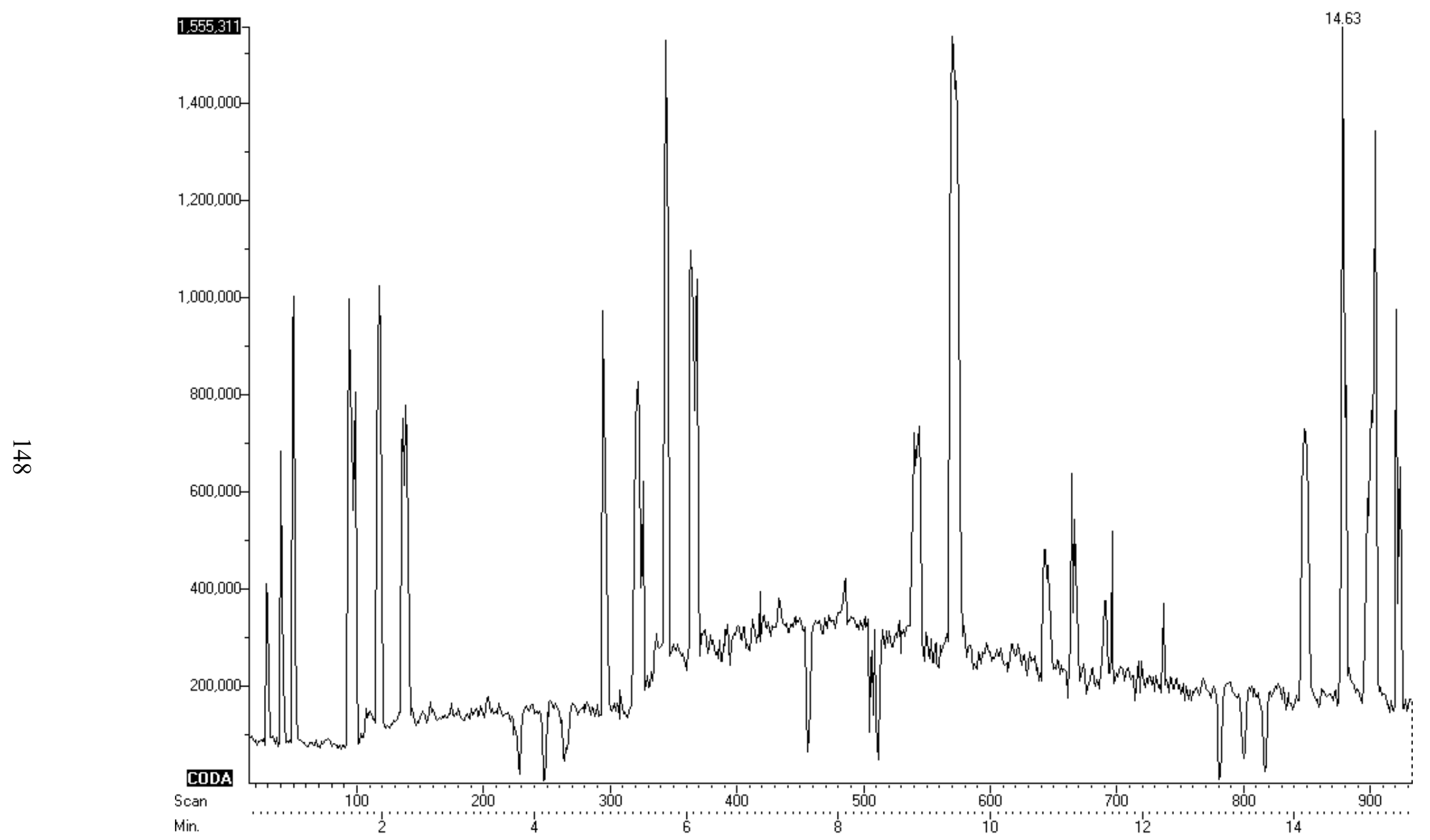

Figure 95 - RIC 8 hours 


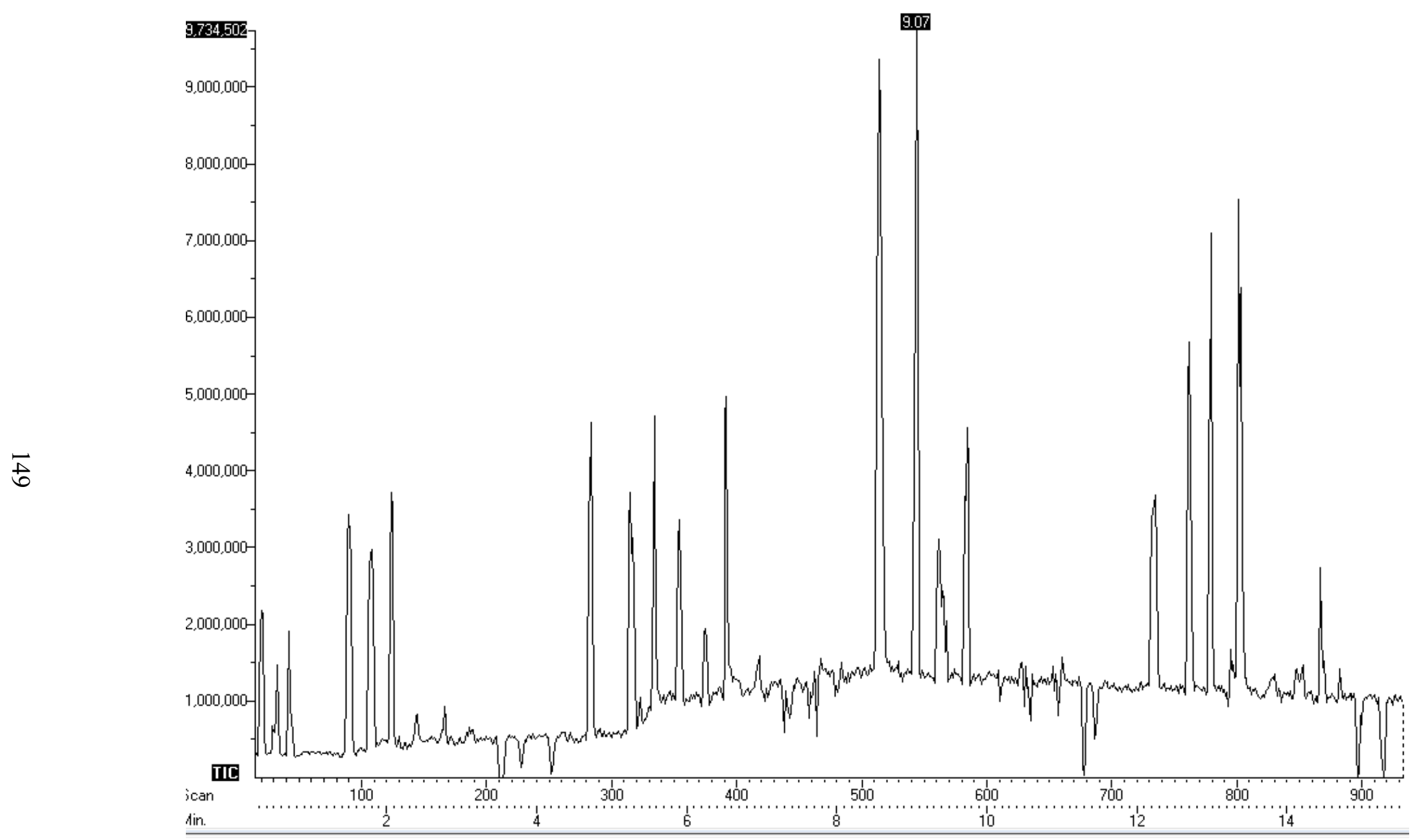

Figure 96 - RIC 10 hours 


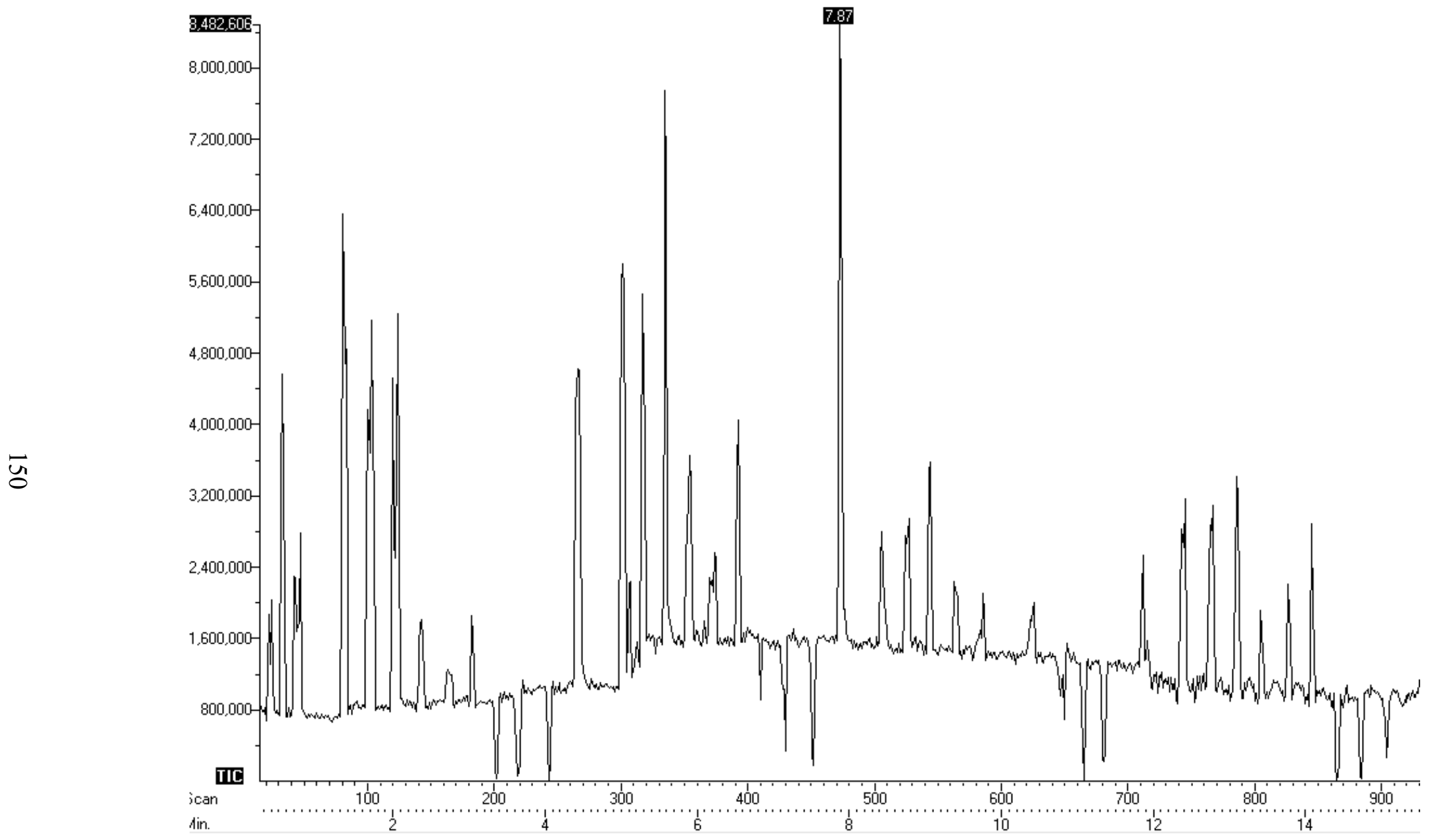

Figure 97 - PIC 12 hours 


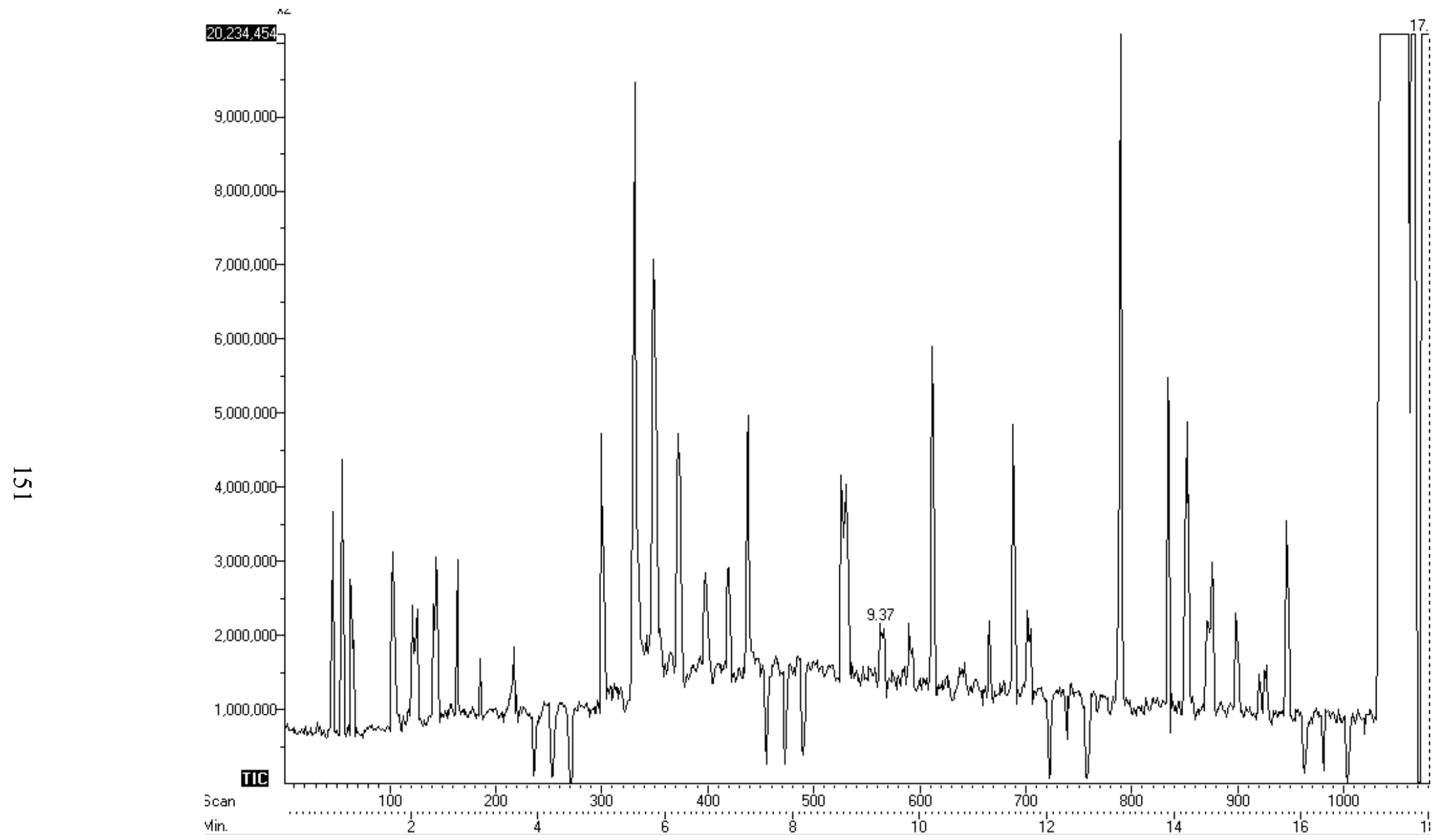

Figure 98 - RIC 14 hours 


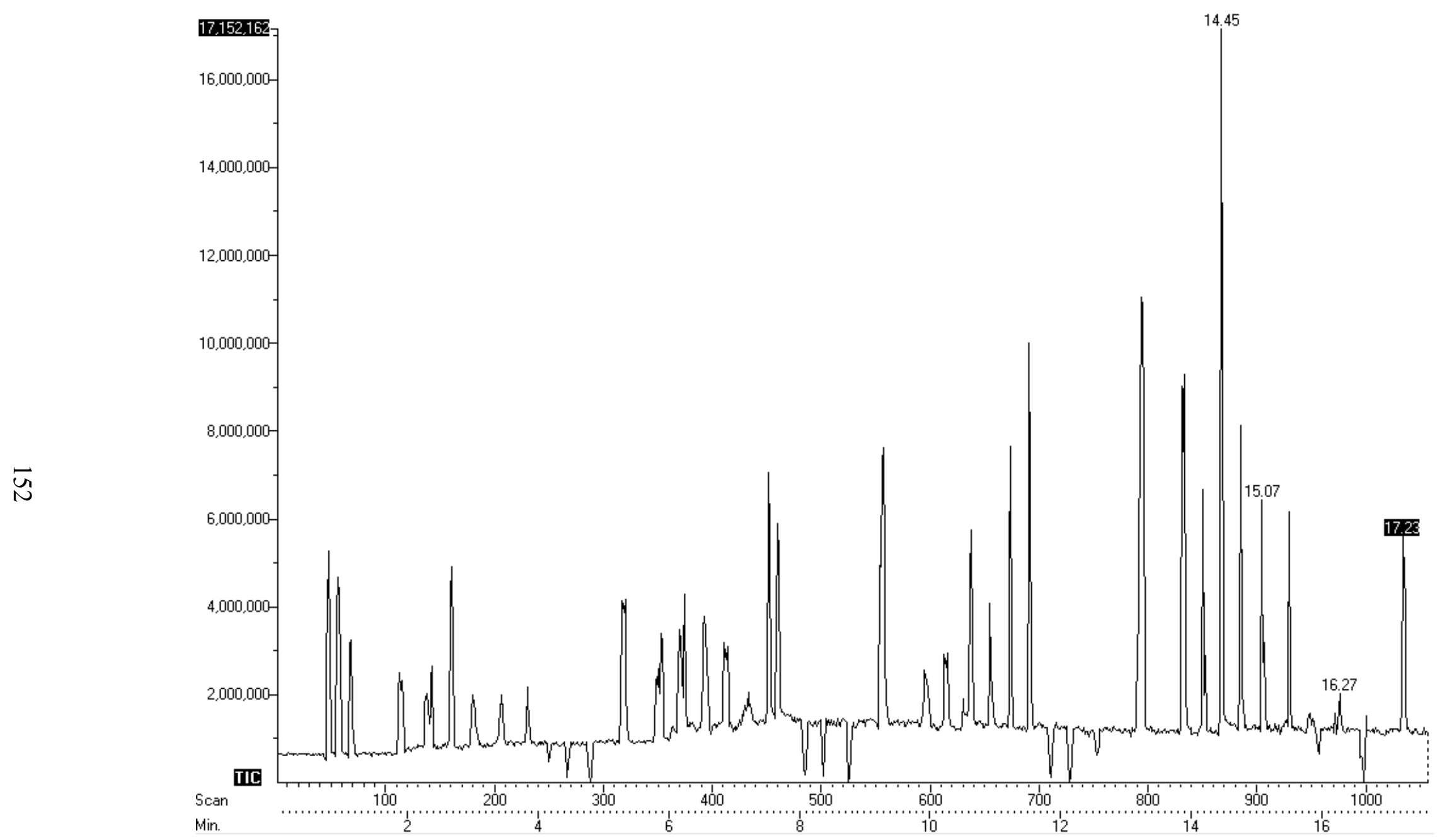

Figure 99 - RIC 16 hours 


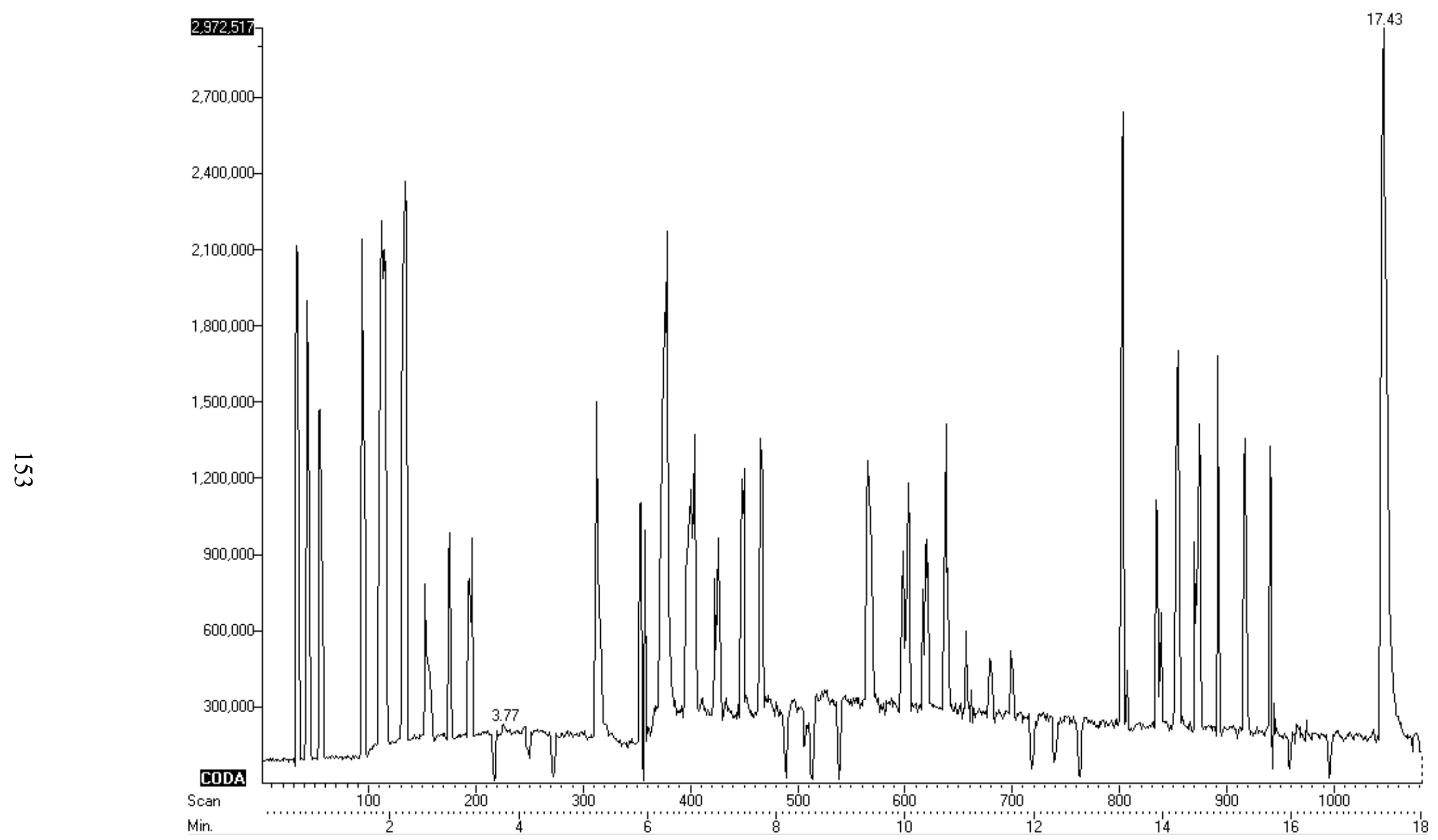

Figure 100 - RIC 18 hours 


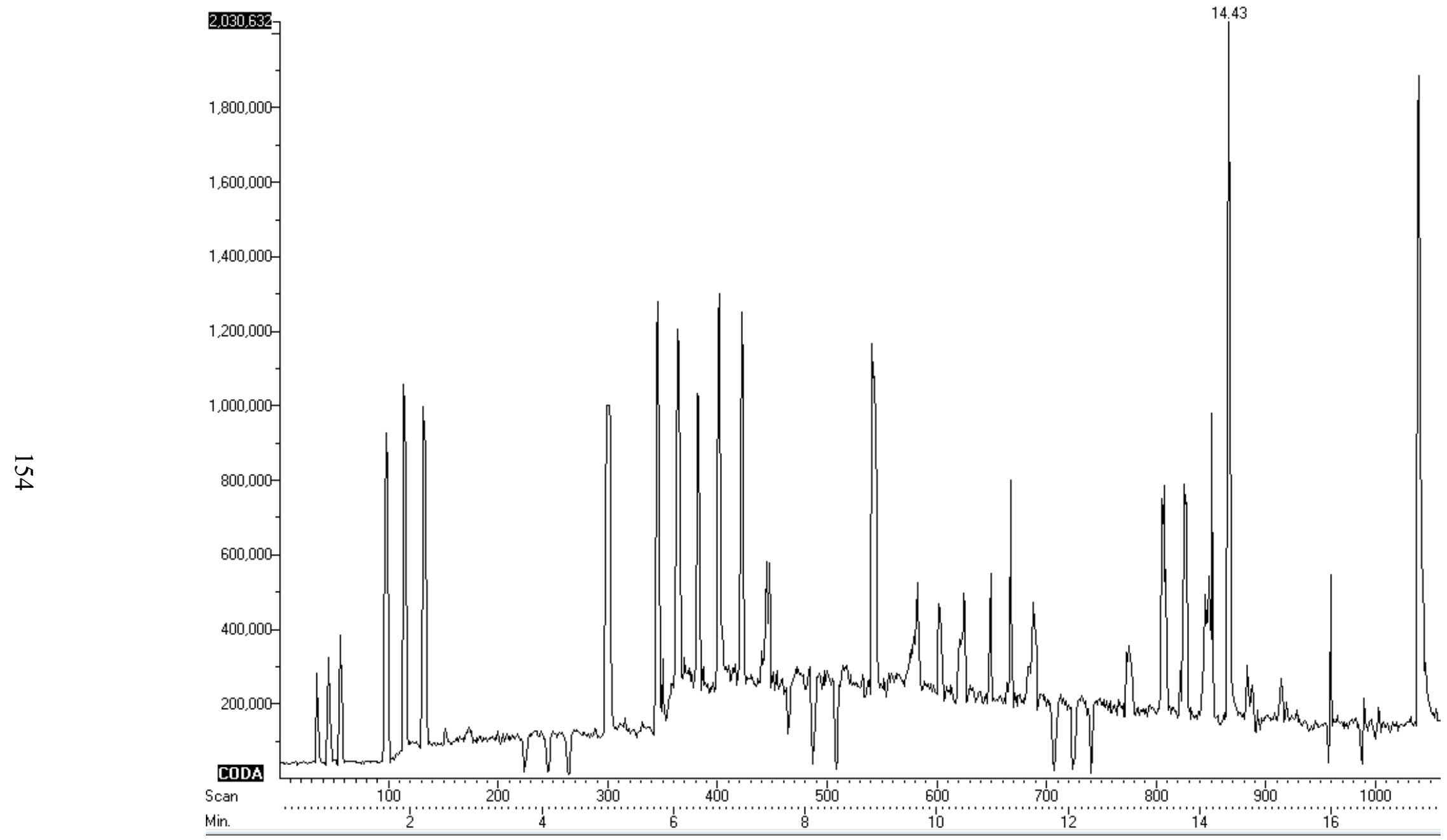

Figure 101 - RIC 20 hours. 


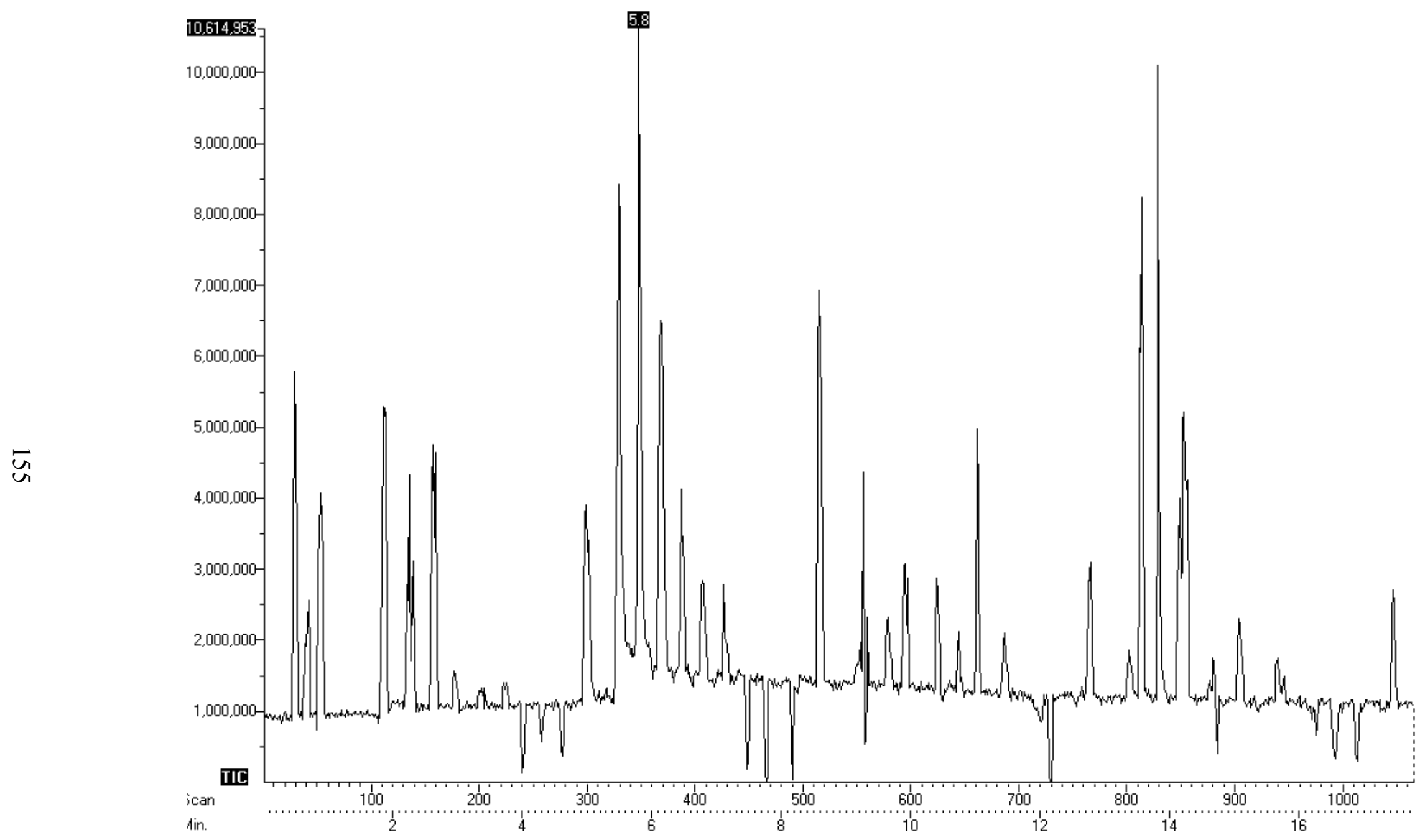

Figure 102 - RIC 22 hours. 


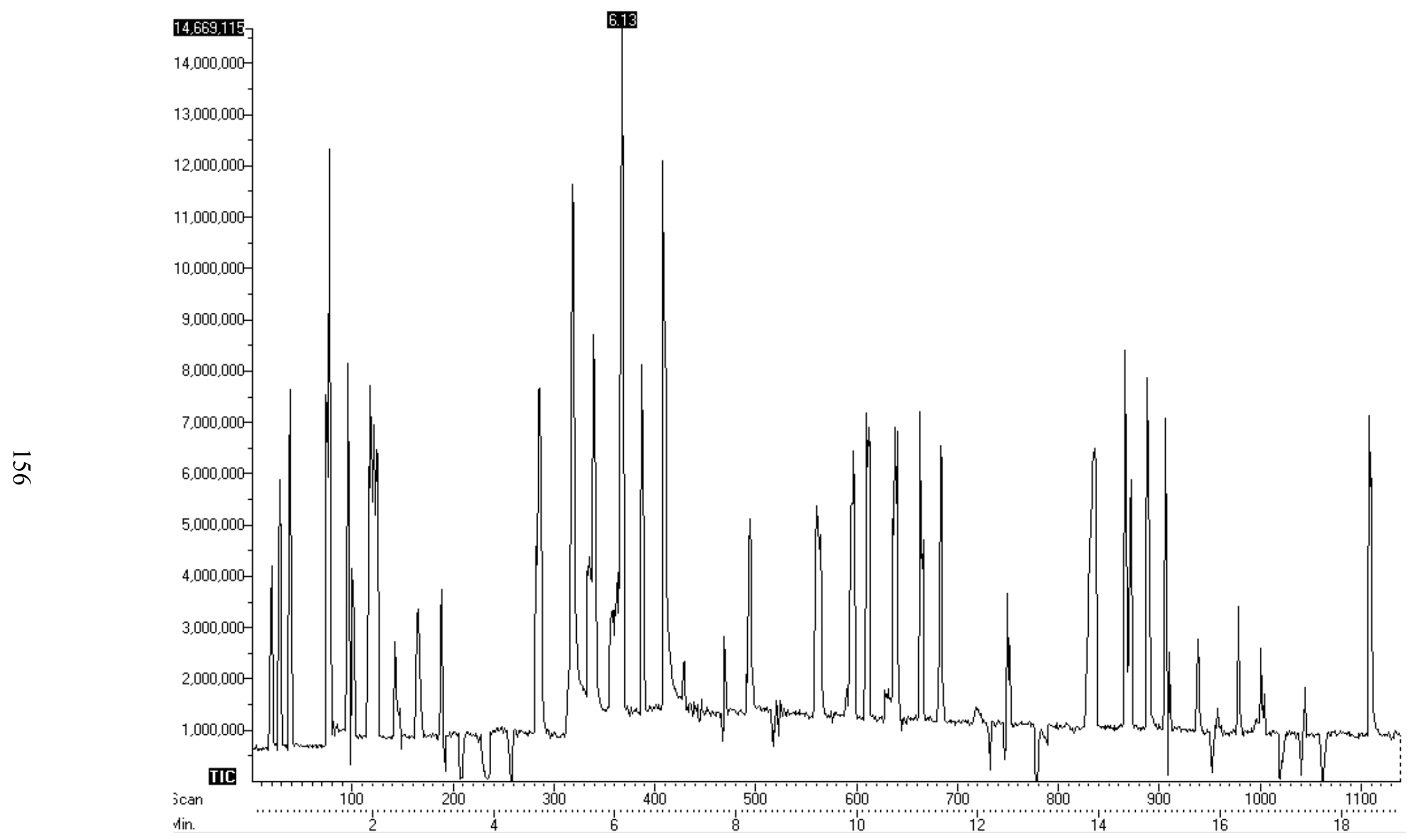

Figure 103 - RIC 24 hours 


\section{Methyl Benzoate}

Methyl benzoate was detected on toothpicks in all the exposure times. It was also only detected on glass capillary tubes after 6 hours of exposure, all the way to 24 hours. It was not detected in any instance at any exposure time on the metal paper clips. For each sample, the intensity was recorded in a spreadsheet (Table 23) and represented visually in Figure 104. The intensity is calculated when a peak is averaged and is shown in the mass spectrum (Figure 105).

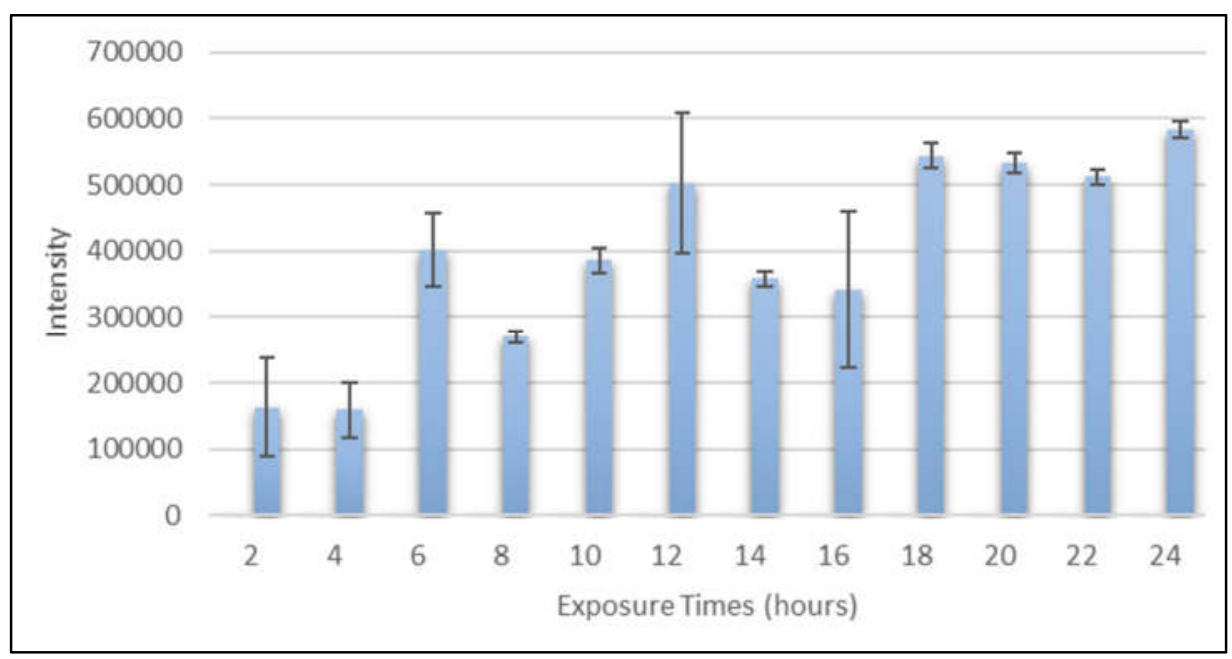

Figure 104 - Visual representation of data from Table 23. 
Table 23 - Intensities for samples tested for methyl benzoate toothpick experiment. Along with the calculated average intensity at each 2 hour interval and its standard deviation.

\begin{tabular}{|c|c|c|c|c|c|}
\hline \multicolumn{5}{|c|}{ Hour } & \multicolumn{3}{c|}{ Intensity } & Average & Std. Dev. \\
\hline 2 & 163,093 & 238,846 & 88,618 & 163,519 & 75,115 \\
\hline 4 & 182,856 & 184,169 & 111,152 & 159,392 & 41,783 \\
\hline 6 & 419,768 & 446,688 & 338,921 & 401,792 & 56,087 \\
\hline 8 & 266,474 & 280,581 & 264,404 & 270,486 & 8,803 \\
\hline 10 & 403,135 & 364,247 & 390,204 & 385,862 & 19,804 \\
\hline 12 & 595,797 & 524,604 & 386,304 & 502,235 & 106,523 \\
\hline 14 & 365,461 & 346,021 & 362,357 & 357,946 & 10,444 \\
\hline 16 & 266,905 & 279,289 & 478,295 & 341,496 & 118,633 \\
\hline 18 & 553,224 & 557,432 & 521,384 & 544,013 & 19,710 \\
\hline 20 & 514,341 & 540,507 & 543,428 & 532,759 & 16,017 \\
\hline 22 & 509,772 & 522,997 & 502,575 & 511,781 & 10,358 \\
\hline 24 & 581,282 & 596,400 & 573,197 & 583,626 & 11,778 \\
\hline
\end{tabular}




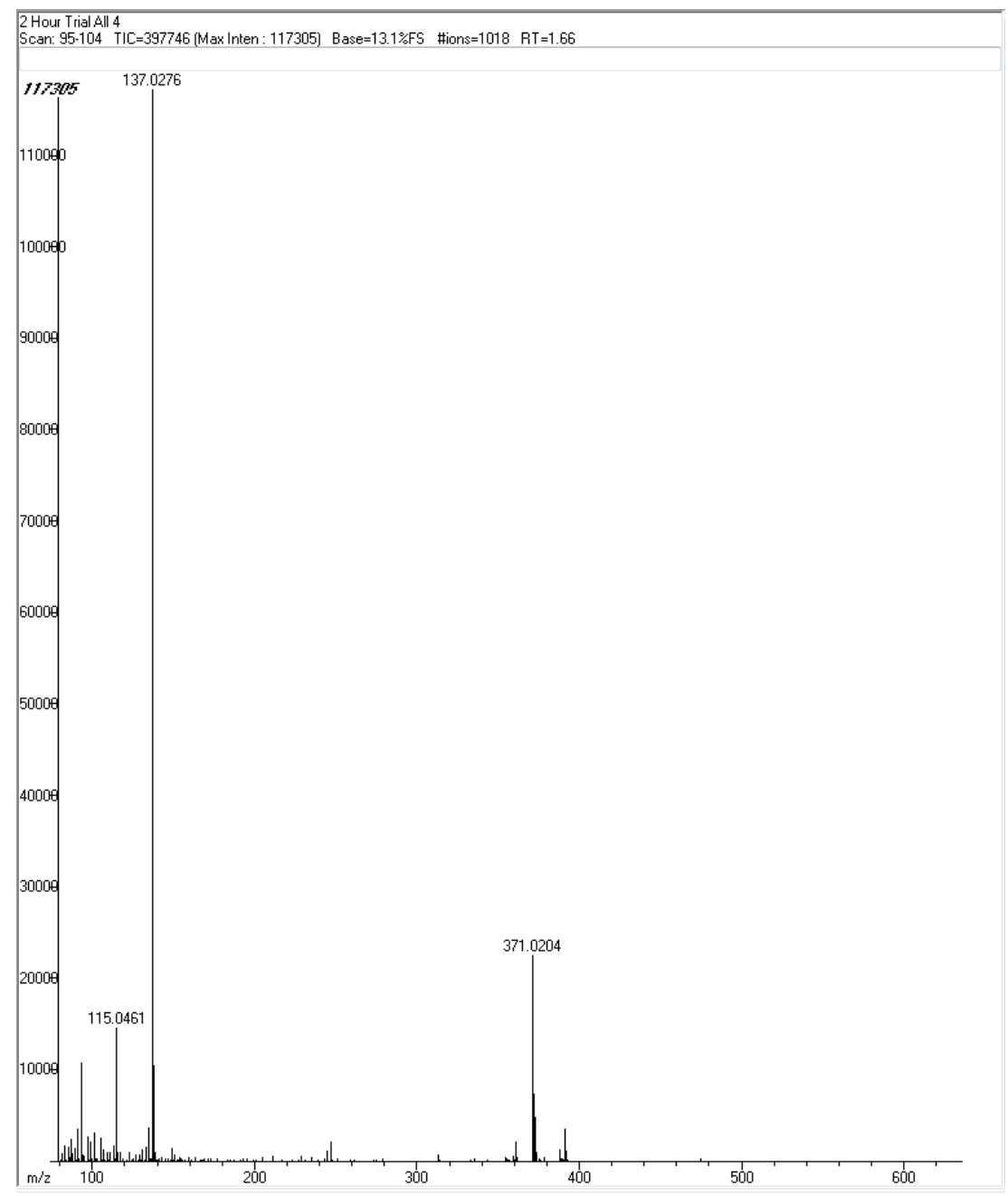

Figure 105 - Mass spectrum of methyl benzoate (m/z 137) toothpick sample \#1 (From RIC shown in Figure 92, $4^{\text {th }}$ peak from the left). The intensity is shown at the top (Max Inten)

Results for methyl benzoate and capillary glass tubes are shown in Table 24. Again, none of the trials prior to 6 hours of exposure time produced any results. But it consistently produced results at all intervals between 6 and 24 hours. Unfortunately, none of the 36 trials using metal paper clips produced any positive results showing the presence of methyl benzoate. 
Table 24 - Intensities for samples tested for methyl benzoate glass experiment. Along with the calculated average intensity at each 2 hour interval and its standard deviation.

\begin{tabular}{|c|c|c|c|c|c|}
\hline Hour & $\# 1$ & $\begin{array}{c}\text { nsensity } \\
\# 2\end{array}$ & $\# 3$ & Average & Std. Dev. \\
\hline 2 & DND & DND & DND & N/A & N/A \\
\hline 4 & DND & DND & DND & $\mathrm{N} / \mathrm{A}$ & N/A \\
\hline 6 & 47,086 & 56,078 & 64,622 & 55,929 & 8,769 \\
\hline 8 & 58,186 & 58,531 & 65,162 & 60,626 & 3,932 \\
\hline 10 & 52,673 & 45,671 & 47,742 & 48,695 & 3,597 \\
\hline 12 & 62,272 & 66,924 & 46,000 & 58,399 & 10,987 \\
\hline 14 & 76,927 & 67,824 & 56,902 & 67,218 & 10,026 \\
\hline 16 & 51,469 & 39,968 & 55,323 & 48,920 & 7,989 \\
\hline 18 & 55,942 & 52,207 & 34,293 & 47,481 & 11,573 \\
\hline 20 & 43,681 & 49,553 & DND & 46,617 & 27,074 \\
\hline 22 & 37,712 & 35,880 & 47,106 & 40,233 & 6,023 \\
\hline 24 & 49,008 & 42,611 & 42,735 & 44,785 & 3,658 \\
\hline
\end{tabular}

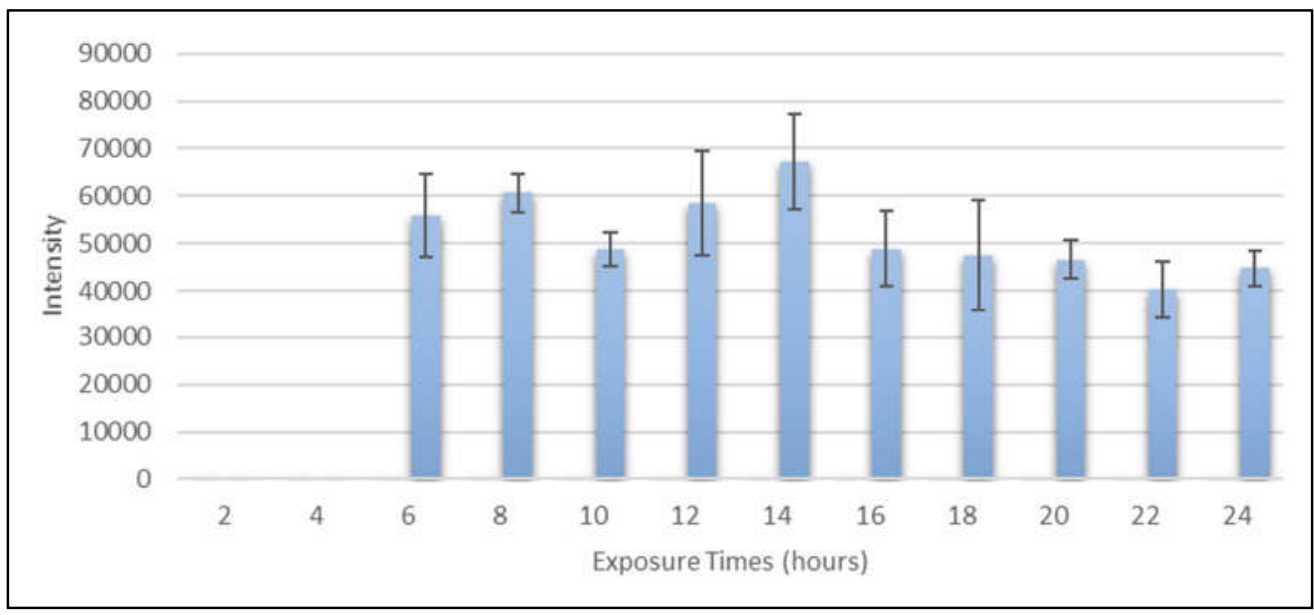

Figure 106 - Visual representation of data from Table 24.

Benzaldehyde

Similarly to methyl benzoate, all toothpick samples exposed to benzaldehyde were identified to contain benzaldehyde when analyzed (Table 25). The average intensities are plotted in Figure 107. But unlike methyl benzoate, benzaldehyde was detected on the capillary glass tubes at each of the 12 time intervals at least once (Table 26), albeit at much 
lower intensities. A sample mass spectrum for benzaldehyde detected on glass is shown in Figure 109.

Table 25 - Intensities for samples tested for benzaldehyde toothpick experiment. Along with the calculated average intensity at each $\mathbf{2}$ hour interval and its standard deviation.

\begin{tabular}{|c|c|c|c|c|r|}
\hline \multirow{2}{*}{ Hour } & 1 & 2 & 3 & Average & \multicolumn{1}{c|}{ Std. Dev. } \\
\hline 2 & 171,282 & 204,732 & 368,167 & 248,060 & 105,351 \\
\hline 4 & 338,752 & 363,035 & 218,160 & 306,649 & 77,590 \\
\hline 6 & 484,125 & 511,047 & 609,024 & 534,732 & 65,732 \\
\hline 8 & 269,111 & 427,640 & 520,783 & 405,845 & 127,244 \\
\hline 10 & 394,247 & 373,090 & 358,675 & 375,337 & 17,892 \\
\hline 12 & 491,039 & 470,903 & 482,298 & 481,413 & 10,097 \\
\hline 14 & 450,141 & 461,857 & 438,287 & 450,095 & 11,785 \\
\hline 16 & 249,713 & 325,268 & 447,782 & 340,921 & 99,958 \\
\hline 18 & 361,293 & 533,460 & 592,700 & 495,818 & 120,208 \\
\hline 20 & 445,448 & 439,899 & 405,385 & 430,244 & 21,707 \\
\hline 22 & 533,721 & 488,554 & 582,312 & 534,862 & 46,889 \\
\hline 24 & 523,536 & 613,418 & 649,265 & 595,406 & 64,771 \\
\hline
\end{tabular}

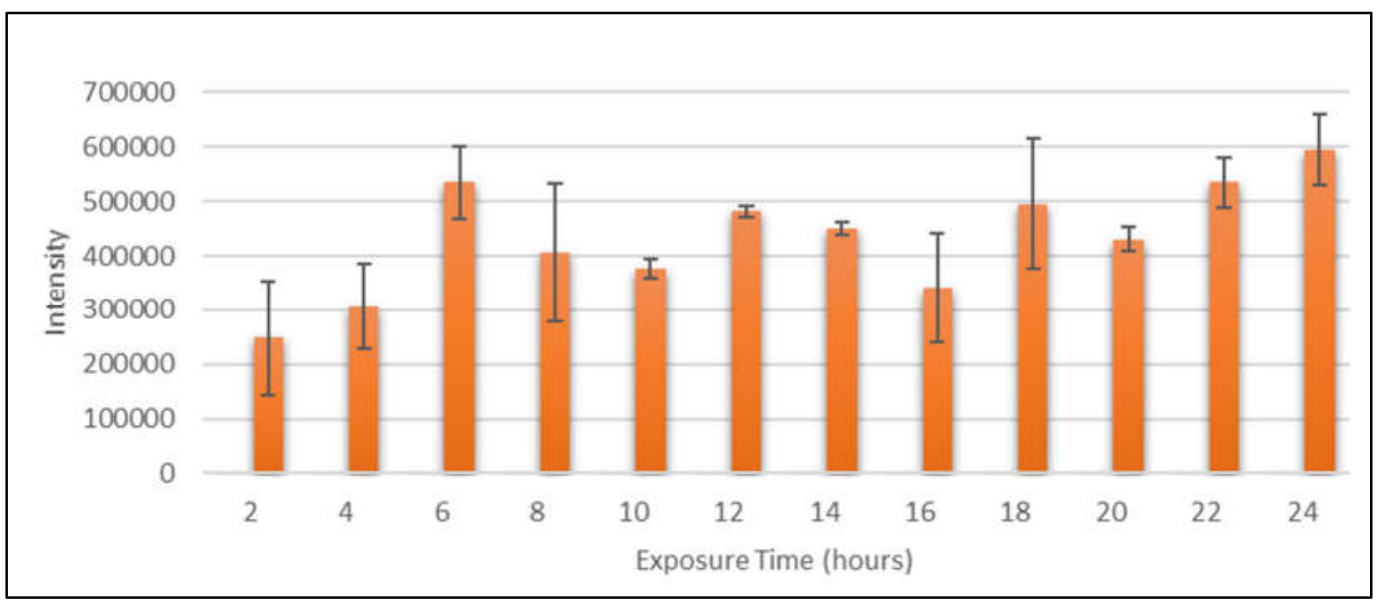

Figure 107 - Visual representation of data from Table 25. 
Table 26 - Intensities for samples tested for benzaldehyde capillary glass experiment. Along with the calculated average intensity at each $\mathbf{2}$ hour interval and its standard deviation.

\begin{tabular}{|c|c|c|c|c|r|}
\hline \multirow{2}{*}{ Hour } & 1 & 2 & 3 & Average & Std. Dev. \\
\hline 2 & 142,668 & 266,235 & 155,913 & 188,272 & 67,842 \\
\hline 4 & 123,846 & DND & 424,793 & 274,320 & 218,463 \\
\hline 6 & 136,687 & 128,962 & DND & 132,825 & 76,783 \\
\hline 8 & 93,454 & 91,517 & DND & 92,486 & 53,405 \\
\hline 10 & 124,742 & 319,326 & 137,795 & 193,954 & 108,771 \\
\hline 12 & 369,276 & 162,770 & 136,690 & 222,912 & 127,424 \\
\hline 14 & 117,827 & 123,299 & 382,995 & 208,040 & 151,540 \\
\hline 16 & 390,084 & DND & DND & 390,084 & N/A \\
\hline 18 & 53,441 & 51,000 & 78,990 & 61,144 & 15,503 \\
\hline 20 & 185,085 & 132,985 & 117,722 & 145,264 & 35,320 \\
\hline 22 & 91,678 & 91,418 & 92,526 & 91,874 & 579 \\
\hline 24 & 74,199 & 115,583 & 141,262 & 110,348 & 33,837 \\
\hline
\end{tabular}

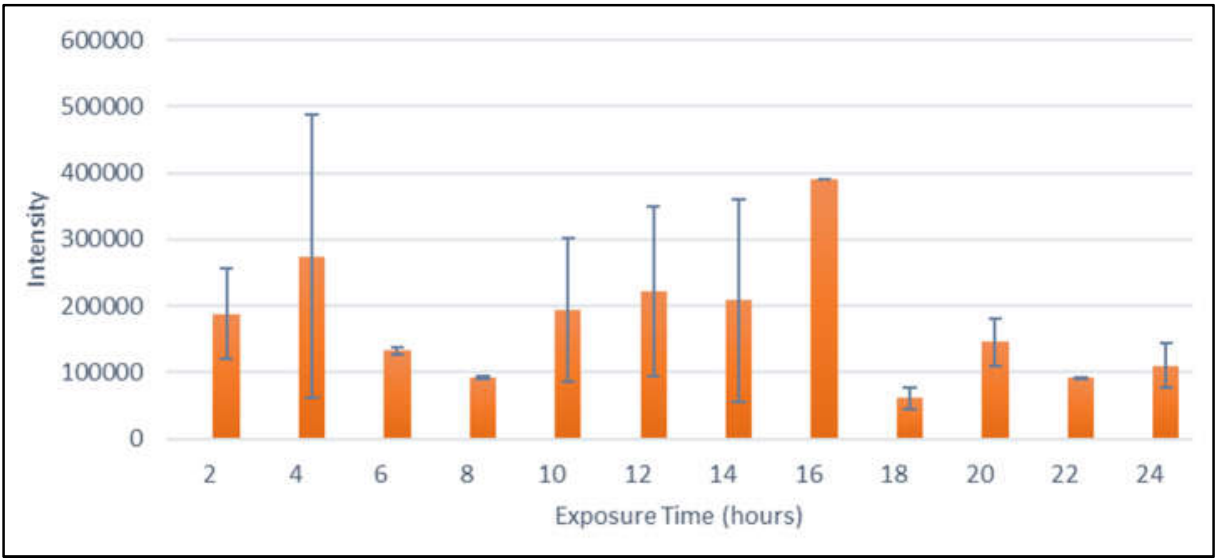

Figure 108 - Visual representation of data from Table 26. 


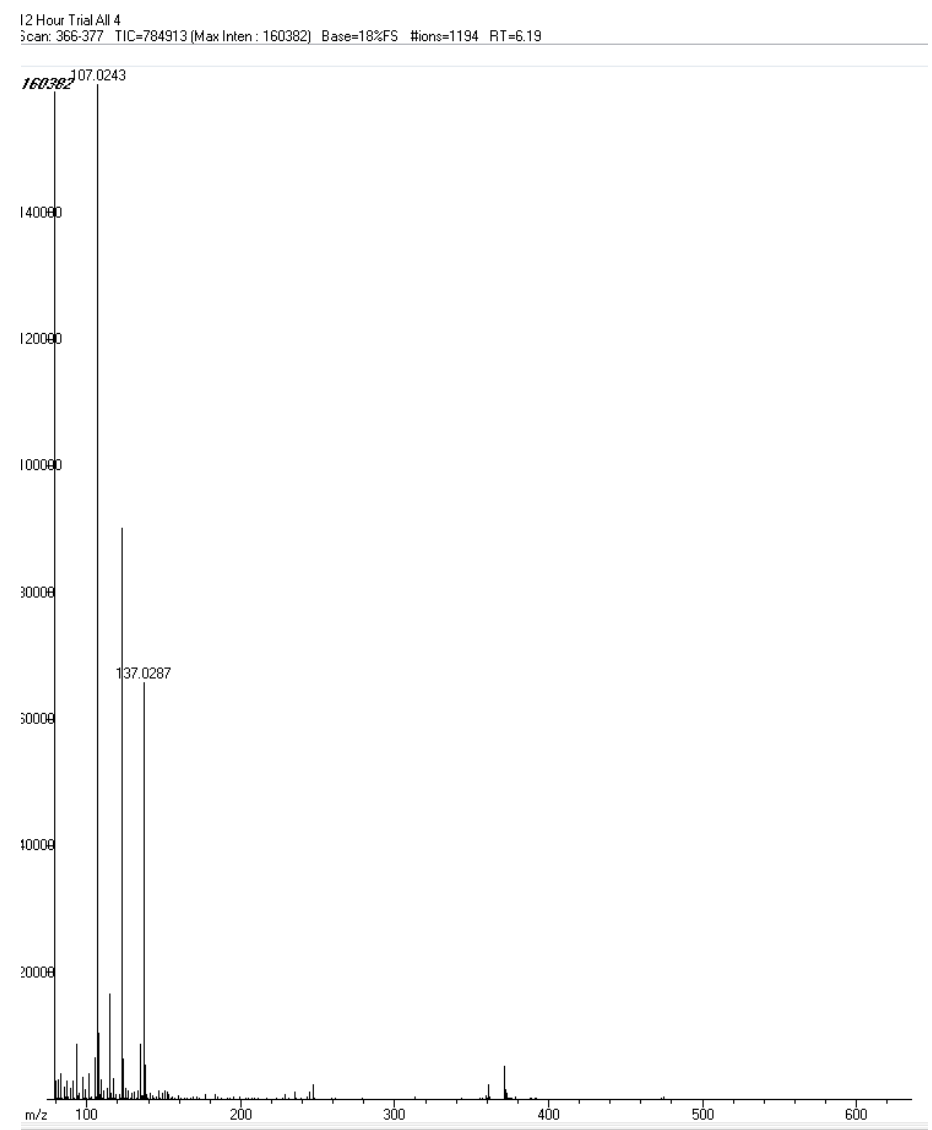

Figure 109 - Mass spectrum of the glass sample \#2 exposed to benzaldehyde (m/z 107). (From RIC shown in Figure 97, $18^{\text {th }}$ peak from the left).

Metal paper clips produced results at only one time interval, the final 24 hour mark.

Two of the three samples were identified to contain benzaldehyde when analyzed. The intensities were 168,611 and 233,256 respectively (average 200,934 $\pm 45,710$ ). For perspective, a chart was plotted to compare the averaged intensities of all three substrates (Figure 110). 


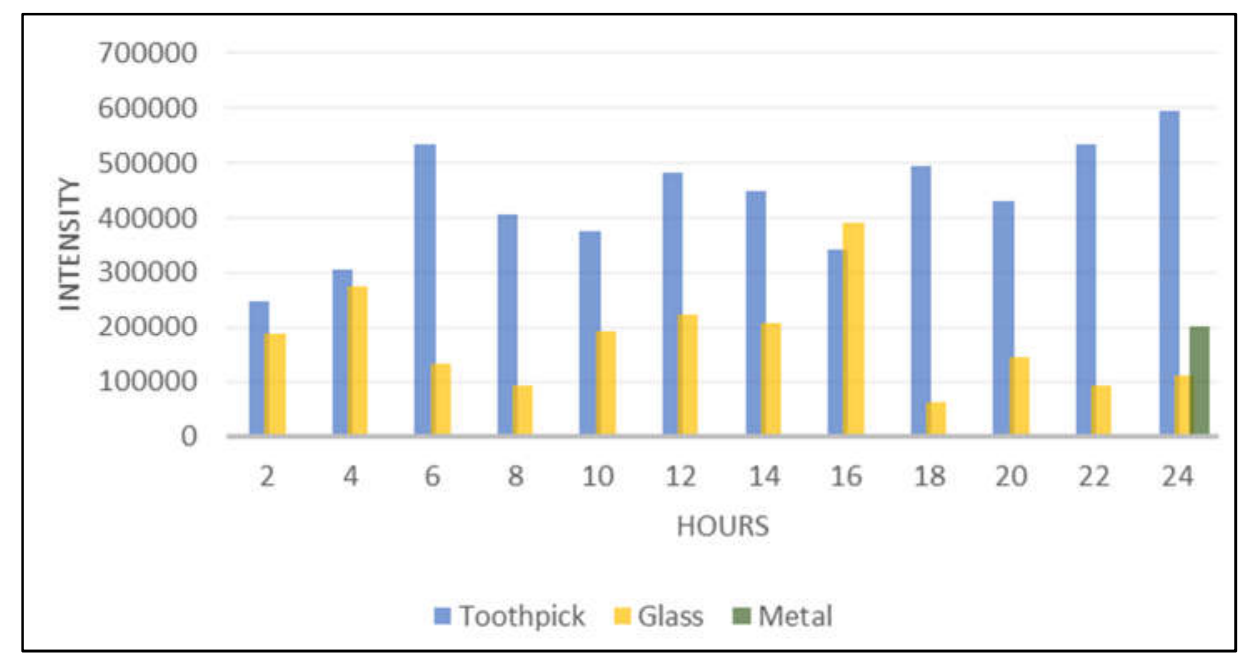

Figure 110 - Chart comparing the averaged intensities for toothpick, glass and metal clip data (benzaldehyde).

\section{2-ethyl-1-hexanol}

With toothpicks, 2-ethyl-hexanol was detected on all but one trial (Sample \#1 @ 10 hour exposure time (Table 27)). Of all the results, all three samples exposed for 12 hours stands out as potentially being an outlier, as the intensities are orders of magnitude higher than the rest of the results. What's unexpected, is that the intensities are relatively similar between the three samples. When retested, it was found that the samples must have been contaminated, since intensities that high only occur when the liquid is directly sampled into the DART stream, as direct sampling the headspace directly was not enough to generate intensities that high either. The updated results are shown in Table 27 - Intensities for samples tested for 2-ethyl-1-hexanol toothpick experiment. Along with the calculated average intensity at each 2 hour interval and its standard deviation.. 
Table 27 - Intensities for samples tested for 2-ethyl-1-hexanol toothpick experiment. Along with the calculated average intensity at each 2 hour interval and its standard deviation.

\begin{tabular}{|c|c|c|c|c|r|}
\hline \multirow{2}{*}{ Hour } & 1 & 2 & 3 & Average & Std. Dev. \\
\hline 2 & 15,633 & 5,701 & 17,038 & 12,791 & 6,180 \\
\hline 4 & 85,546 & 16,171 & 13,741 & 38,486 & 40,773 \\
\hline 6 & 9,584 & 10,124 & 10,311 & 10,006 & 378 \\
\hline 8 & 19,327 & 25,895 & 8,474 & 17,899 & 8,798 \\
\hline 10 & DND & 28,545 & 49,410 & 38,978 & 24,804 \\
\hline 12 & 200,799 & 220,338 & 239,941 & 220,359 & 19,571 \\
\hline 14 & 14,317 & 8,597 & 12,944 & 11,953 & 2,986 \\
\hline 16 & 15,248 & 18,704 & 18,654 & 17,535 & 1,981 \\
\hline 18 & 77,929 & 22,973 & 52,462 & 51,121 & 27,503 \\
\hline 20 & 13,560 & 13,443 & 14,931 & 13,978 & 827 \\
\hline 22 & 60,493 & 12,886 & 24,731 & 32,703 & 24,785 \\
\hline 24 & 28,498 & 8,304 & 48,105 & 28,302 & 19,901 \\
\hline
\end{tabular}

With glass, 2-ethyl-1-hexanol was detected on four occasions. Once after 8 hours of exposure (9,153 relative intensity), once after 22 hours of exposure (17,386 relative intensity), and twice after 24 hours of exposure (3,513 and 68,964 relative intensity). Whereas with the metal clips were not detected at any trial during any of the exposure times. For comparison purposes, the averaged intensities for all 2-ethyl-1-hexanol samples were plotted in Figure 111. When looking at that chart, the discrepancy of the 12 hour toothpick results become more obvious. Yet even if those results were to be omitted, the remaining results do not show a pattern of slow progression in intensity as expected. 


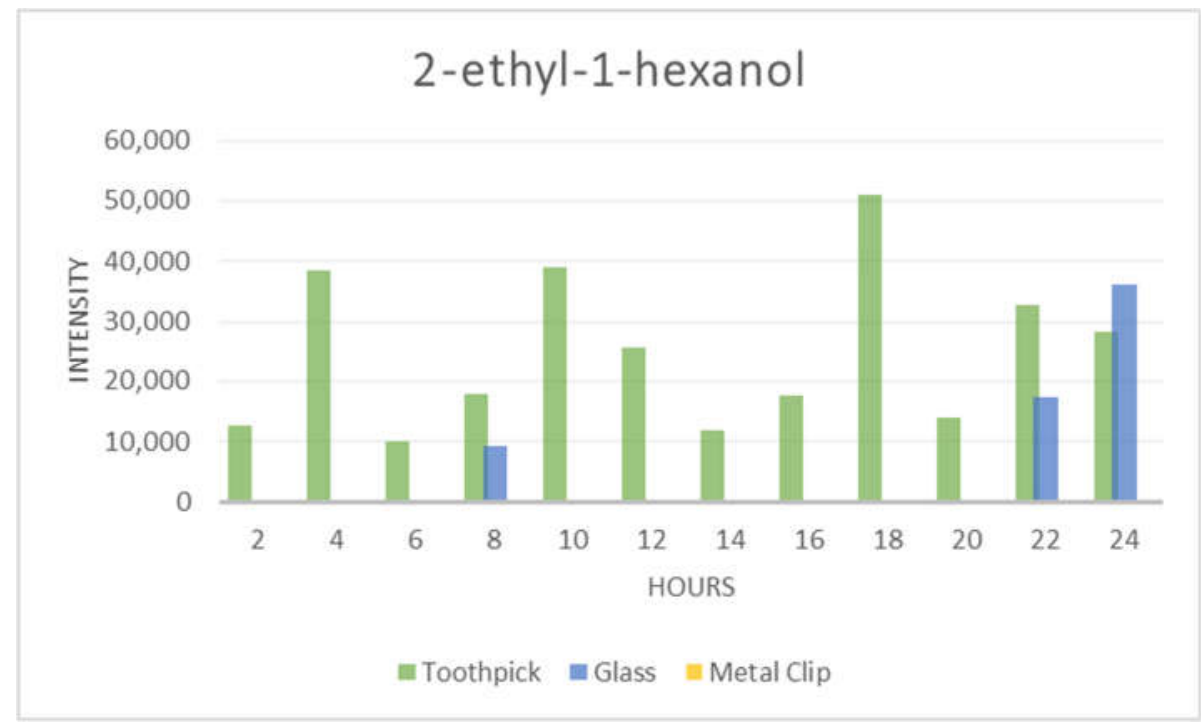

Figure 111 - Chart comparing results for toothpick, glass and metal clips exposed to 2ethyl-1-hexanol for 24 hours at 2 hour intervals.

Unlike methyl benzoate and benzaldehyde, when 2-ethyl-1-hexanol is identified, its dimer peak is never the parent ion peak. Therefore, determining the intensity isn't as straight forward as the other two chemicals. Once the peak from the RIC is averaged and $\mathrm{m} / \mathrm{z} 261$ is observed, the relative intensity is determined by looking for that specific $\mathrm{m} / \mathrm{z}$ on the right side of the mass spectrum, as shown in Figure 112. 


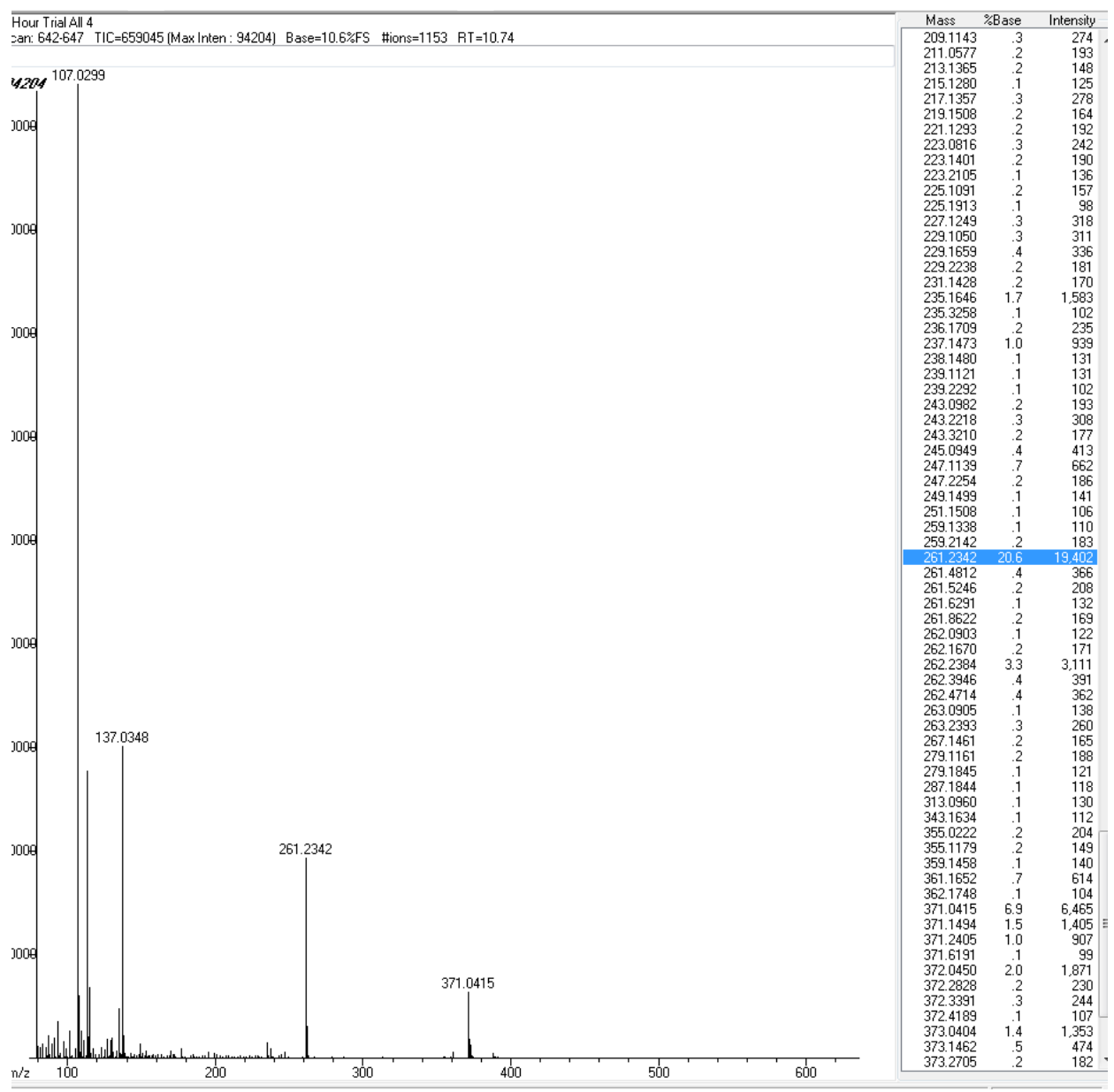

Figure 112 - Mass spectrum of the toothpick sample \#1 exposed to 2-ethyl-1-hexanol for 8 hours. (From RIC shown in Figure 95, $4^{\text {th }}$ peak from the left). Since $\mathrm{m} / \mathrm{z} 261$ is not the parent ion peak in this mass spectrum, its relative intensity is shown on the table to the right of the mass spectrum.

\section{Diethyl Pthalate}

Of the four chemical standards, diethyl phthalate has been the most successful in terms of results. In the toothpick experiment, diethyl phthalate was detected in every trial for every time interval in this study (Table 28), similarly to methyl benzoate and benzaldehyde. It was also detected in every trial for every time interval with glass ( 
Table 29), which was not observed with any of the other chemical standards. Yet unlike the other chemicals, diethyl phthalate produced tangible results when testing with the metal paper clips.

Table 28 - Intensities for samples tested for diethyl phthalate toothpick experiment. Along with the calculated average intensity at each 2 hour interval and its standard deviation.

\begin{tabular}{|r|r|r|r|r|r|}
\hline \multirow{5}{*}{ Hour } & \multicolumn{1}{c}{ Sample \# } & \multicolumn{1}{c|}{ Average } & Std. Dev. \\
\hline 2 & 44,431 & 237,583 & 114,603 & 132,206 & 97,772 \\
\hline 4 & 108,467 & 40,520 & 49,131 & 66,039 & 36,995 \\
\hline 6 & 34,347 & 343,393 & 343,423 & 240,388 & 178,436 \\
\hline 8 & 195,403 & 187,475 & 129,714 & 170,864 & 35,857 \\
\hline 10 & 237,896 & 365,512 & 355,057 & 319,488 & 70,854 \\
\hline 12 & 96,151 & 96,736 & 124,198 & 105,695 & 16,027 \\
\hline 14 & 53,433 & 160,872 & 103,366 & 105,890 & 53,764 \\
\hline 16 & 318,561 & 166,695 & 260,454 & 248,570 & 76,627 \\
\hline 18 & 98,505 & 230,936 & 91,337 & 140,259 & 78,610 \\
\hline 20 & 95,779 & 90,830 & 71,919 & 86,176 & 12,592 \\
\hline 22 & 306,397 & 425,374 & 289,122 & 340,298 & 74,183 \\
\hline 24 & 238,537 & 249,486 & 173,249 & 220,424 & 41,220 \\
\hline
\end{tabular}

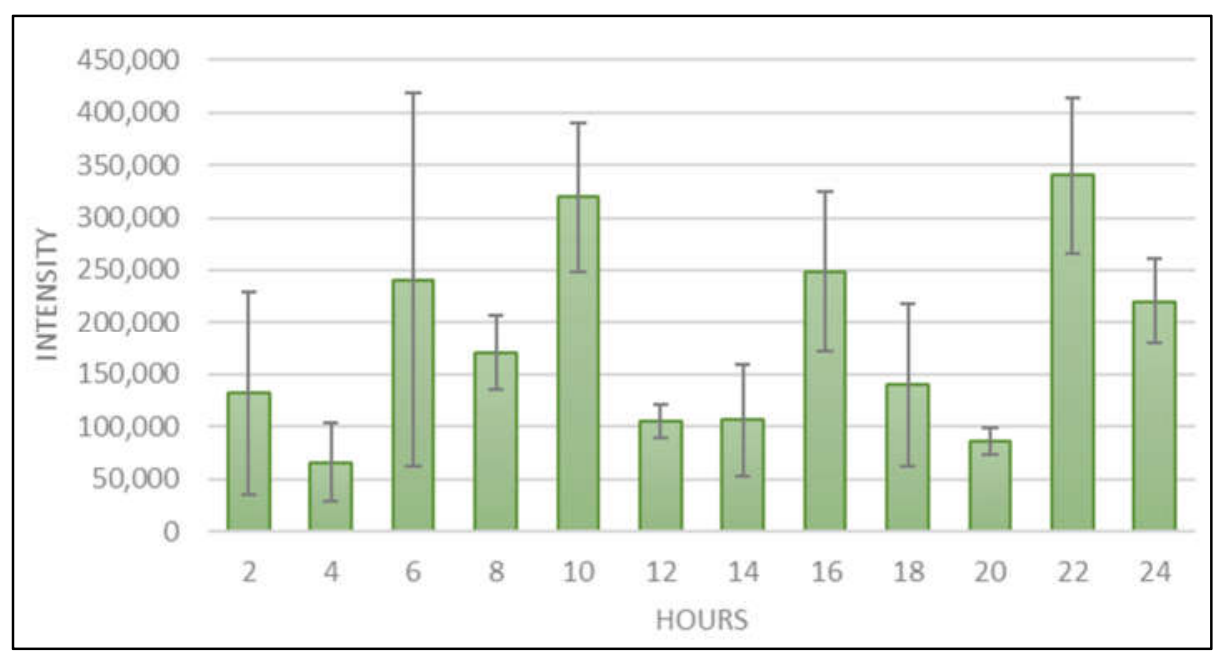

Figure 113 - Visual representation of Table 28

As was the case with 2-ethyl-1-hexanol, the identifying peak in the mass spectrum for diethyl phthalate (which is $[\mathrm{M}+\mathrm{H}]=233$ ) is not the parent ion peak. Therefore, the 
relative intensity needs to be obtained from the table next to the mass spectrum in the software, as highlighted in Figure 114.

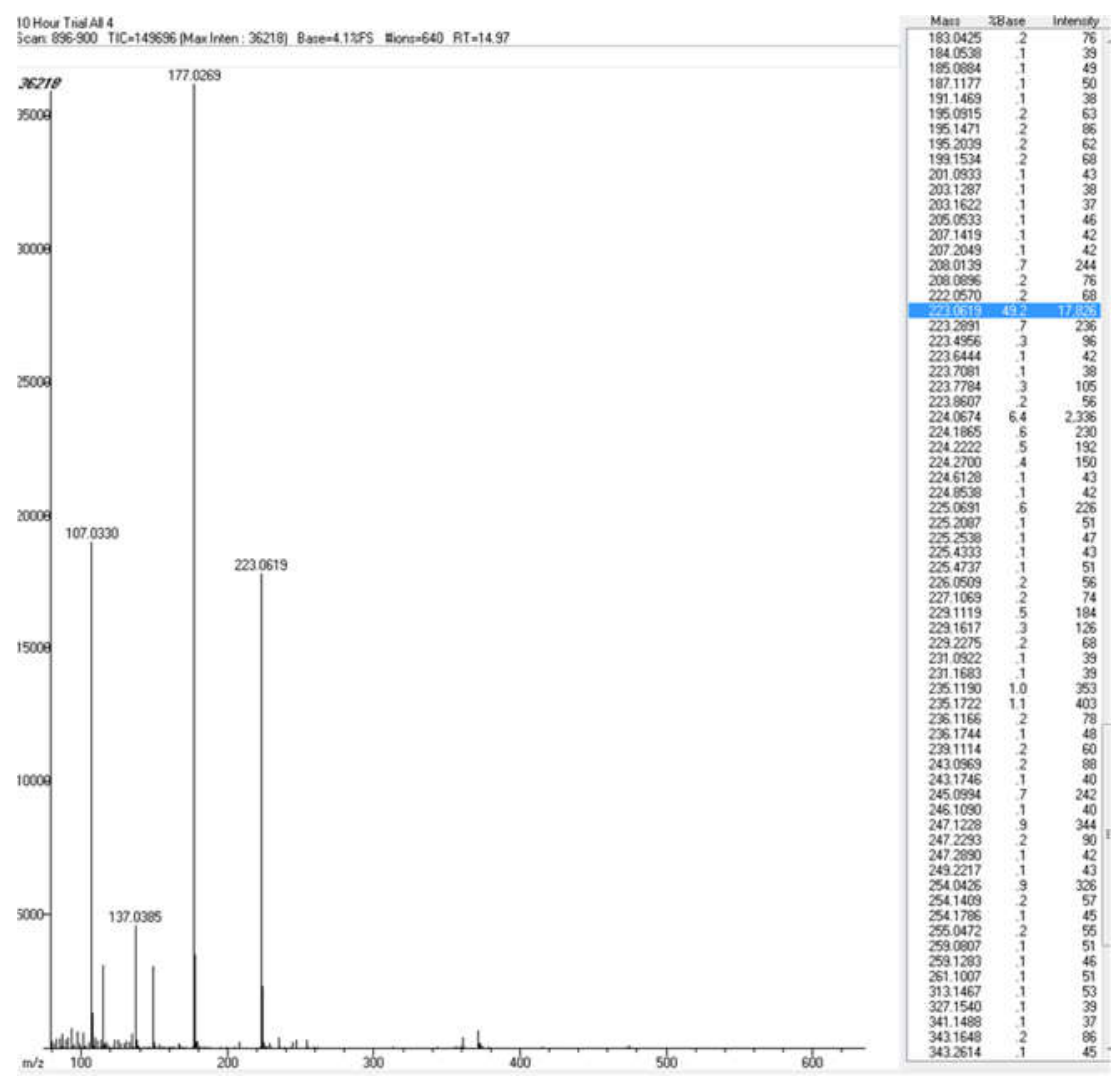

Figure 114 - Mass spectrum of the toothpick sample \#1 exposed to diethyl phthalate for 10 hours. (From RIC shown in Figure 96, $4^{\text {th }}$ peak from the right). Since $\mathrm{m} / \mathrm{z} 233$ is not the parent ion peak in this mass spectrum, its relative intensity is shown on the table to the right of the mass spectrum. 
Table 29 - Intensities for samples tested for diethyl phthalate glass experiment. Along with the calculated average intensity at each $\mathbf{2}$ hour interval and its standard deviation.

\begin{tabular}{|c|c|c|c|c|r|}
\hline \multirow{2}{*}{ Hour } & 1 & 2 & 3 & Average & Std. Dev. \\
\hline 2 & 6,987 & 10,117 & 35,258 & 17,454 & 15,498 \\
\hline 4 & 5,329 & 10,875 & 40,323 & 18,842 & 18,808 \\
\hline 6 & 114,104 & 154,459 & 65,071 & 111,211 & 44,764 \\
\hline 8 & 25,941 & 5,331 & 30,549 & 20,607 & 13,429 \\
\hline 10 & 10,832 & 29,280 & 56,206 & 32,106 & 22,819 \\
\hline 12 & 13,943 & 41,614 & 55,642 & 37,066 & 21,218 \\
\hline 14 & 27,459 & 22,004 & 42,553 & 30,672 & 10,645 \\
\hline 16 & 77,987 & 87,047 & 73,126 & 79,387 & 7,065 \\
\hline 18 & 81,884 & 39,228 & 126,690 & 82,601 & 43,735 \\
\hline 20 & 182,386 & 12,287 & 13,640 & 69,438 & 97,818 \\
\hline 22 & 30,971 & 39,690 & 22,699 & 31,120 & 8,496 \\
\hline 24 & 48,301 & 49,890 & 9,064 & 35,752 & 23,126 \\
\hline
\end{tabular}

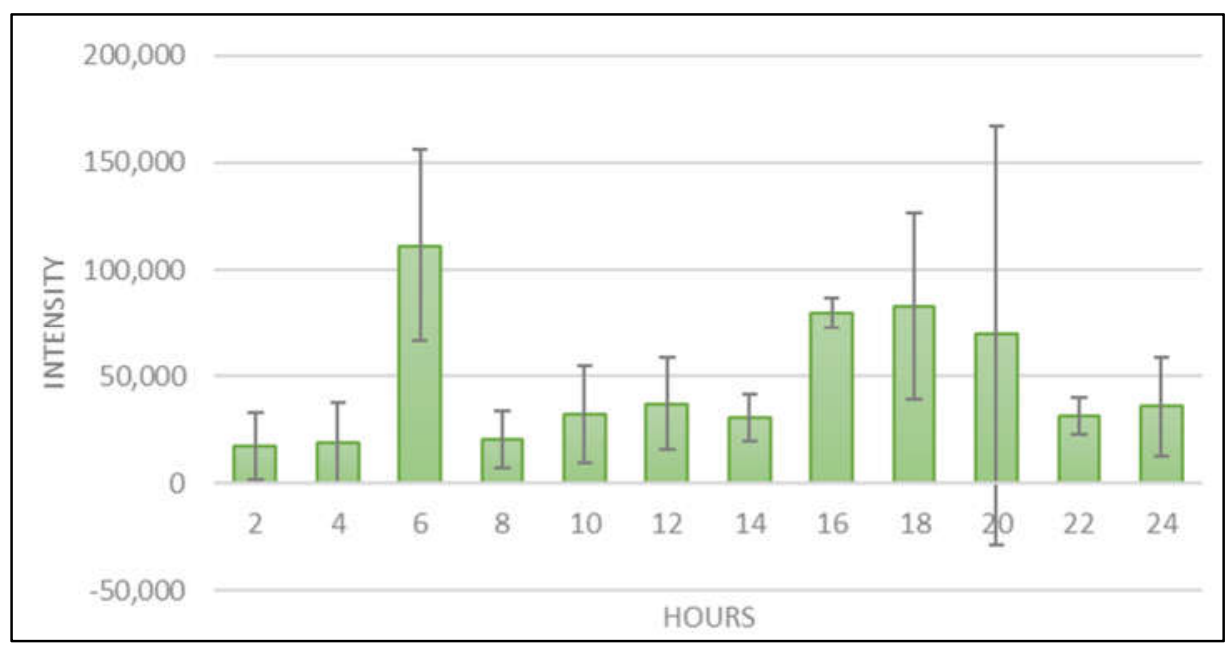

Figure 115 - Visual representation of Table 29.

With benzaldehyde and 2-ethyl-1-hexanol, the chemicals were identified on metal only at with the 24 hour exposure test, and not in one instance with methyl benzoate. However, with diethyl pthalate, it was identified with the metal paper clips at all but three time intervals $(12,14$, and 18 hours). The data for this experiment is shown in Table 30. 
Table 30 - Intensities for samples tested for diethyl phthalate metal experiment. Along with the calculated average intensity at each 2 hour interval and its standard deviation.

\begin{tabular}{|c|c|c|c|c|c|}
\hline Hour & 1 & ample \# & 3 & Average & Std. Dev. \\
\hline 2 & 5,904 & 6,406 & 12,203 & 8,171 & 3,501 \\
\hline 4 & DND & 5,005 & DND & 5,005 & $\mathrm{~N} / \mathrm{A}$ \\
\hline 6 & 32,928 & 36,947 & 89,605 & 53,160 & 31,626 \\
\hline 8 & 11,368 & 20,259 & 13,449 & 15,025 & 4,650 \\
\hline 10 & 17,948 & 5,317 & 7,166 & 10,144 & 6,822 \\
\hline 12 & DND & DND & DND & N/A & N/A \\
\hline 14 & DND & DND & DND & $\mathrm{N} / \mathrm{A}$ & N/A \\
\hline 16 & 10,373 & 21,091 & 17,486 & 16,317 & 5,454 \\
\hline 18 & DND & DND & DND & N/A & N/A \\
\hline 20 & 37,078 & 11,632 & 11,986 & 20,232 & 14,590 \\
\hline 22 & 9,053 & DND & DND & 9,053 & N/A \\
\hline 24 & 6,785 & 29,253 & 4,047 & 13,362 & 13,830 \\
\hline
\end{tabular}

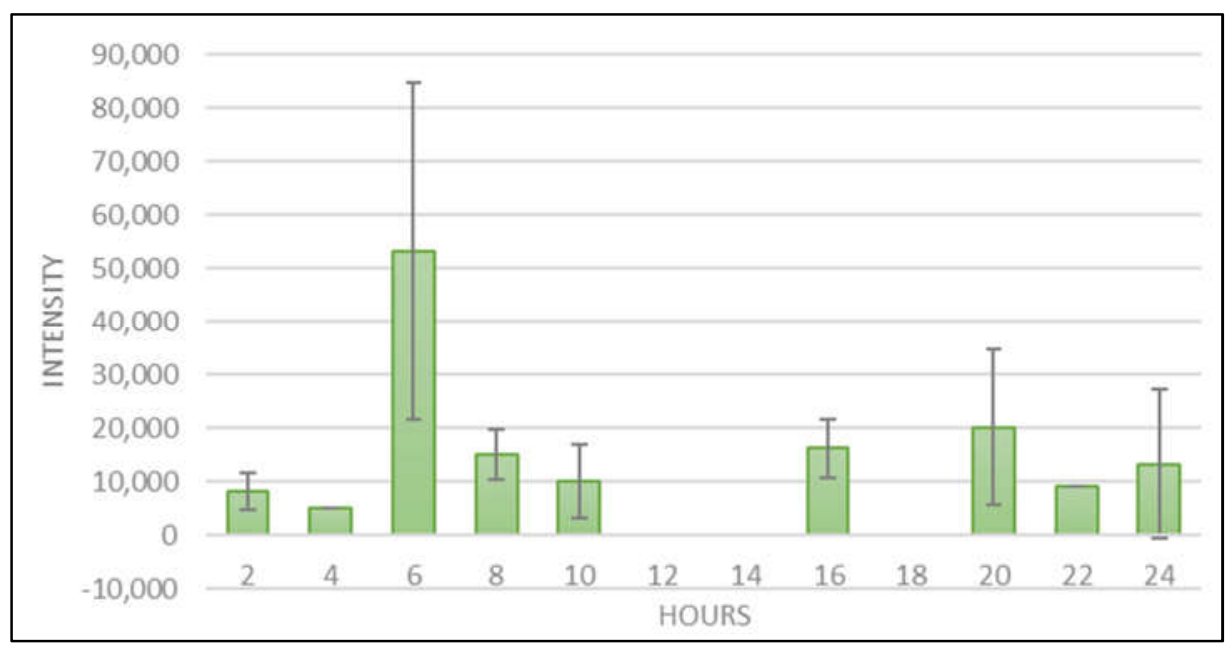

Figure 116 - Visual representation of Table 30.

Overall, these results highlight a major drawback when using the DART for this type of study. Although care was taken from the user's end to try and introduce each sample in the same manner, an attempt at reproducibility, the reality is there are several uncontrollable variables that limit the instrument's ability to produce reproducible quantitative results. These limitations are not only an issue on the hardware end, but on the 
software end as well. Figure 117 shows a zoomed-in area of a RIC, where the peak is highlighted by the user (indicated by the dotted lines), and Figure 118 is the resulting mass spectrum from averaging that area. When looking at the max intensity at the top of the mass spectrum, it shows as 131,668.

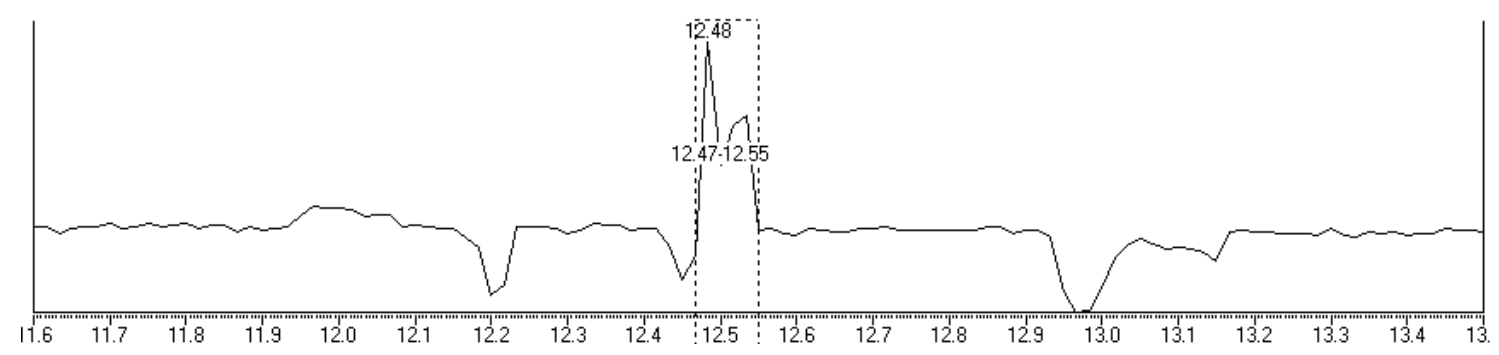

Figure 117 - Zoomed in RIC for 24 hour exposure experiment (full RIC shown in Figure 103). The dotted lines indicate the area selected by the user to average to create a mass spectrum. 


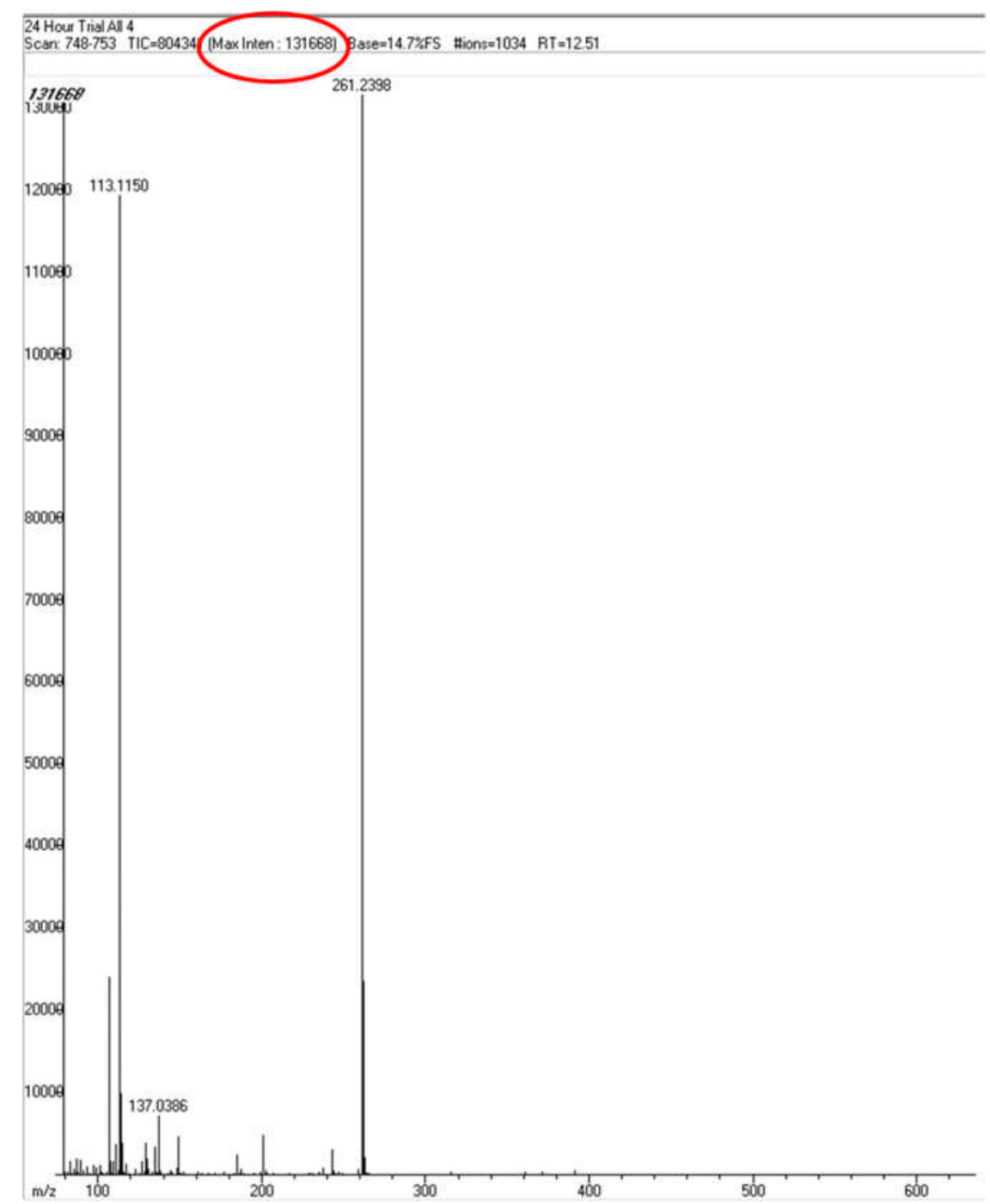

Figure 118 - Mass spectrum of the 2-ethyl-1-hexanol standard in the 24-hour exposure experiment (RIC shown in Figure 103). Max intensity of 131,668. 
However, when attempting to recreate a similar mass spectrum, by averaging the exact same peak, due to a slight difference in area selected as shown in Figure 119, it results in a completely different maximum intensity of 179,888 as shown in Figure 120.

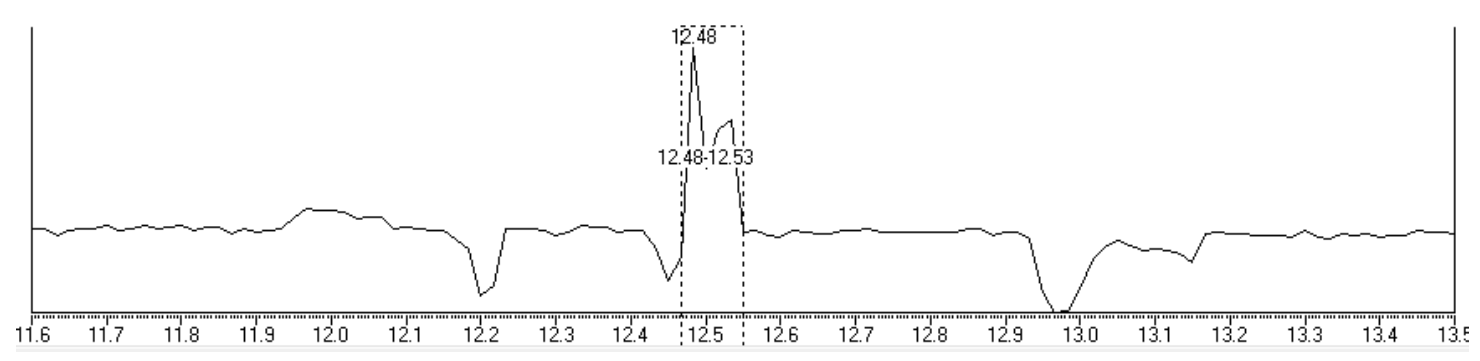

Figure 119 - Zoomed in RIC for 24-hour exposure experiment (full RIC shown in Figure 103). The dotted lines indicate the area selected by the user to average to create a mass spectrum. Note the slight differences between this figure and Figure 117 in the area selected.

Therefore, it is highly improbable that the DART can be used efficiently for quantitative purposes. While the ability to sample in real time, without the need for additional sample preparation, made this instrument ideal for this type of study, the lack of certainty in the quantitative results make it flawed and imperfect.

What this technique does answer is that the instrument can detect the presence of these volatiles on various substrates at various time intervals. The absence of a result does not necessarily mean the volatiles were not present, but rather could have been missed as a result of low concentrations, amounts, or simply due to human error. The effort to mitigate these issues were the reason why all tests were done in triplicate. 


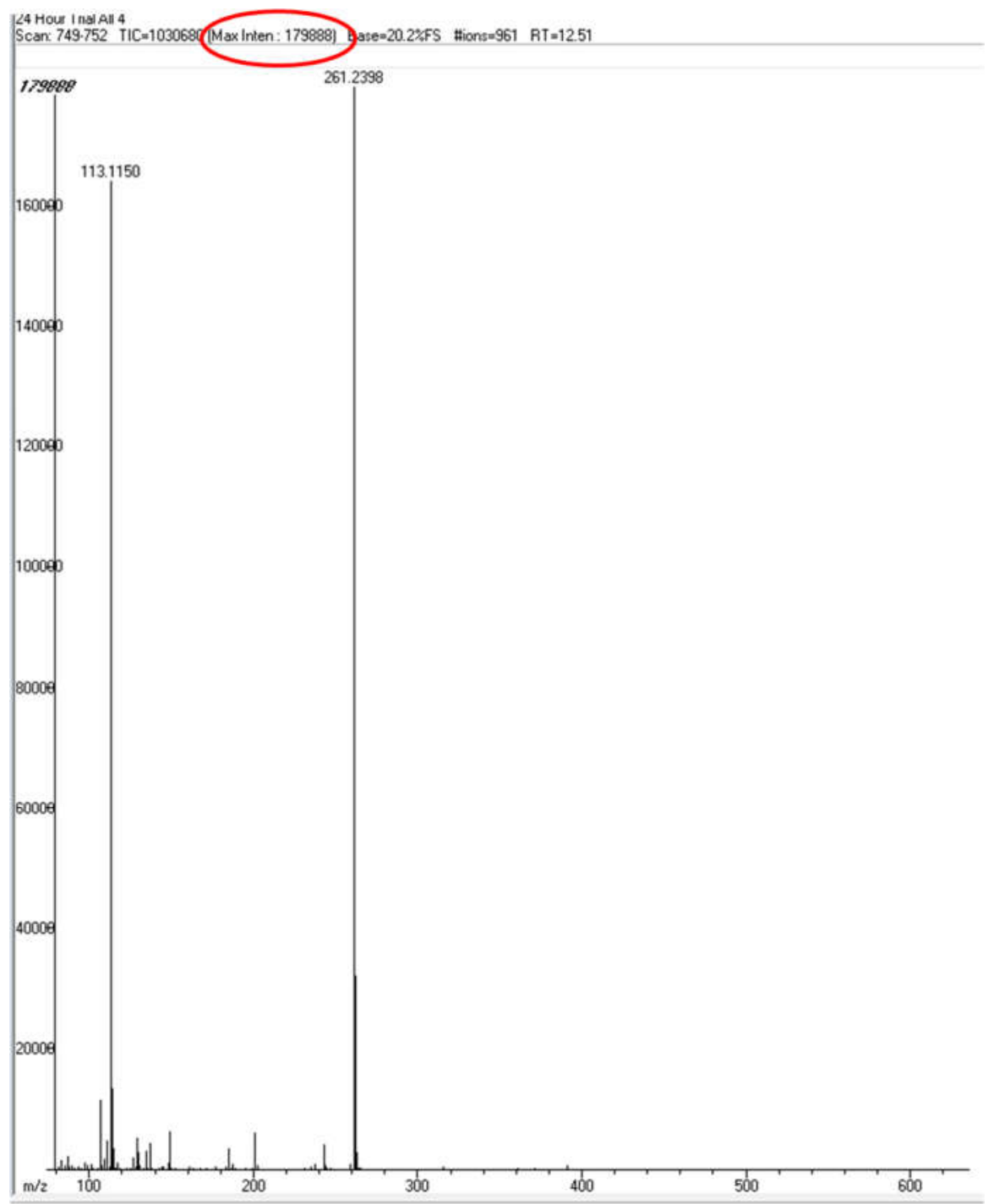

Figure 120 - Mass spectrum of the 2-ethyl-1-hexanol standard in the 24-hour exposure experiment (RIC shown in Figure 103). Max intensity of 179,888. 


\subsection{Task 4: Testing COMPS’ Potential for Cross-Contamination}

This task involved testing the COMPS' primary and secondary containers to see if there is any potential for cross-contamination. The narcotic COMPS differ from the explosive COMPS as they are in a small foil bag (primary container) and a larger foil bag (secondary container). Whereas the explosive mimics are stored in LDEE bags (will refer to as primary container for purposes of this study) placed in mason jars (secondary container).

\section{Narcotic COMPS}

Three narcotic COMPS were tested: (1) cocaine, (2) methamphetamine and (3) MDMA. The secondary container was tested for five minutes, while rotating the bag frequently and trying to get different parts of the bag sampled directly. At the end of each test, the chemical standard of interest is sampled directly into the DART stream to serve as a positive control. None of the three narcotic COMPS exhibited any sign of cross contamination (Table 31 )

Table 31 - Results for narcotic COMPS secondary container cross-contamination test.

$$
(x=\text { not detected, } \checkmark=\text { detected })
$$

\begin{tabular}{|c|c|c|c|}
\hline \multirow{2}{*}{ COMP } & \multicolumn{3}{c|}{ Trial \# } \\
\hline Cocaine & $\mathbf{x}$ & $\mathbf{x}$ & $\mathbf{x}$ \\
\hline Methamphetamine & $\mathbf{x}$ & $\mathbf{x}$ & $\mathbf{x}$ \\
\hline MDMA & $\mathbf{x}$ & $\mathbf{x}$ & $\mathbf{x}$ \\
\hline
\end{tabular}




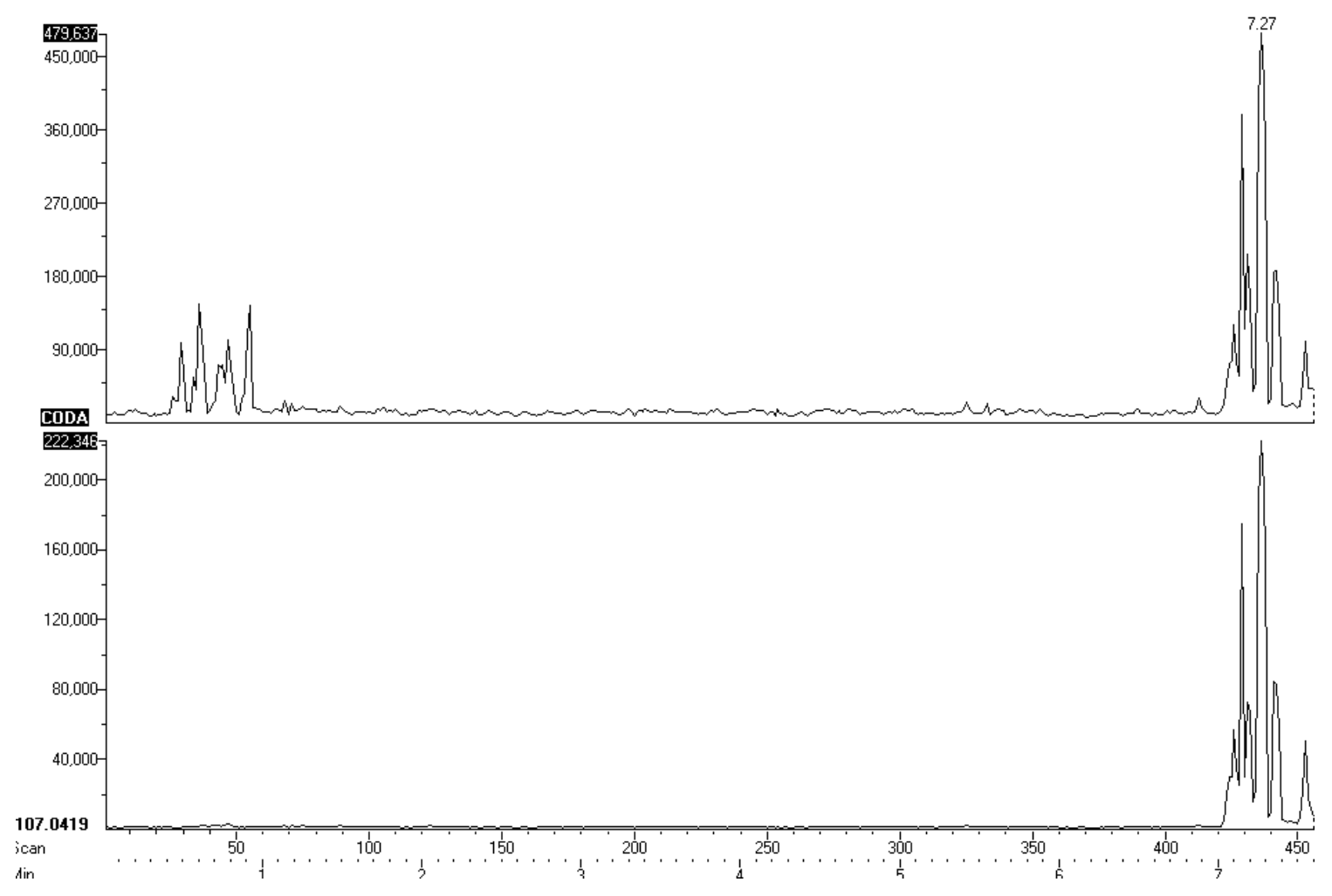

Figure 121 - TOP: RIC for methamphetamine COMPS secondary container test. First series of peaks are PEG calibrant peaks. Last series of peaks are benzaldehyde standard. Bottom: RIC for $\mathrm{m} / \mathrm{z} 107$ that shows it was not detected at any point during the run, with the exception of the standard.

For the primary container, the results were rather mixed. Prior to opening these bags by ripping the area below the heat seals, no cross-contamination was detected for any of the three narcotic COMPS. However, after the bags were opened, and even after they were resealed (via press-seal), cross contamination was detected in a few instances (Table 32). An example RIC of when that occurred is shown in in Figure 122.

Upon further examination of the primary containers, it was apparent that the powders within the bag moved around and was sticking to press-seal and was even found in between the flap above. A way to describe the way this occurs would be to think of an old dusty box, where the dust is visibly floating in the area directly above the box. In a 
similar fashion, the dust-like powders used to make these mimics can be seen floating in the air when these bags are opened. Therefore, the use of a secondary container for storage purposes becomes of paramount important to prevent the possibility of cross contamination.

Table 32 - Results for narcotic COMPS primary container cross-contamination test.

$$
(x=\text { not detected, } \checkmark=\text { detected })
$$

\begin{tabular}{|c|c|c|c|}
\hline \multirow{2}{*}{ COMP } & \multicolumn{3}{c|}{ Trial \# } \\
\hline Cocaine & 1 & 2 & 3 \\
\hline Methamphetamine & $\mathbf{x}$ & $\checkmark$ & $\boldsymbol{x}$ \\
\hline MDMA & $\mathbf{x}$ & $\mathbf{x}$ & $\checkmark$ \\
\hline
\end{tabular}

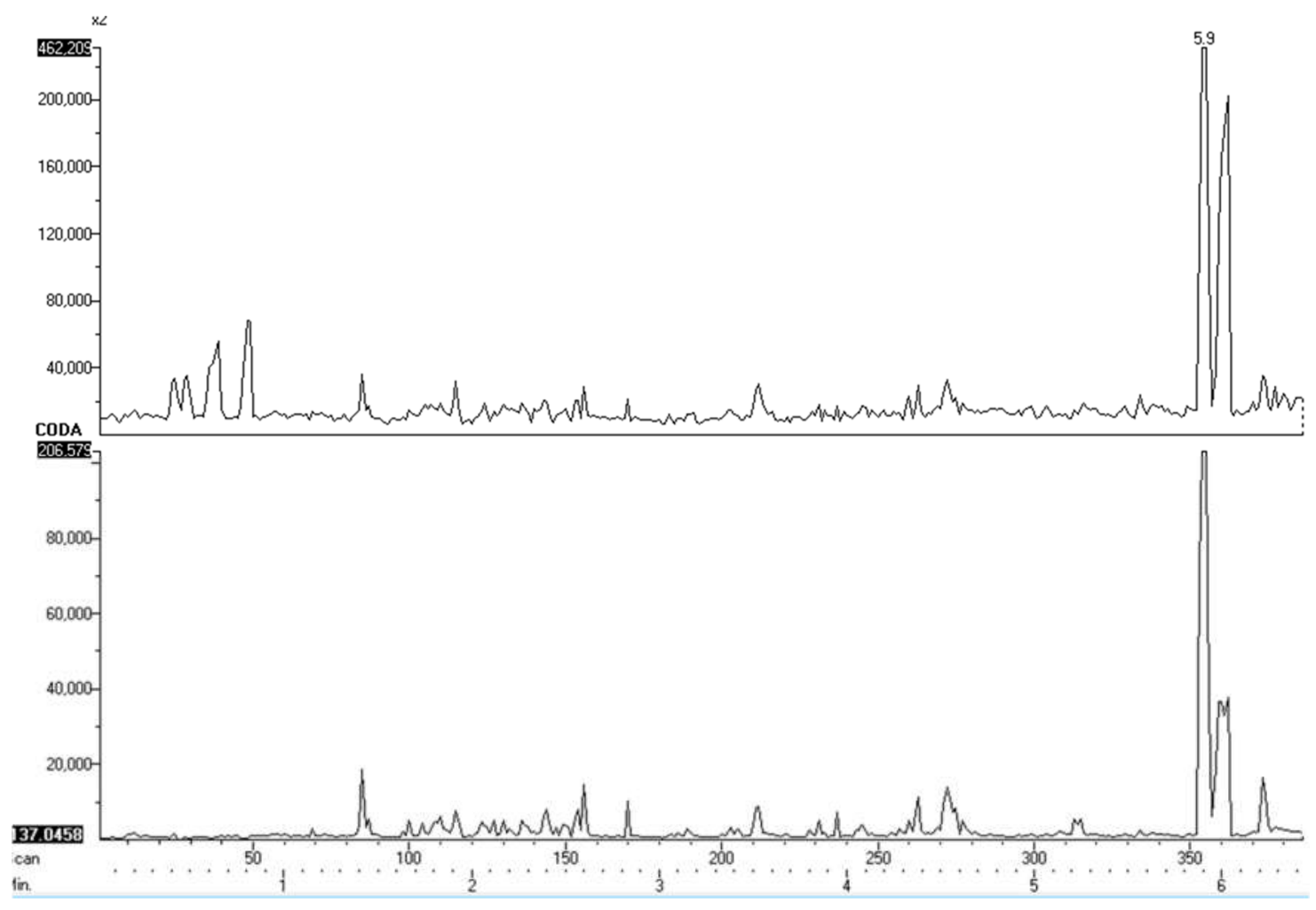

Figure 122 - TOP: RIC for cocaine COMPS primary container test. First series of peaks are PEG calibrant peaks. Last series of peaks are methyl benzoate standard. Bottom: RIC for $\mathrm{m} / \mathrm{z} 137$ that shows it was detected at various points during the run. 


\section{Explosive COMPS}

Unlike the narcotic COMPS, the explosive COMPS are stored in LDPE bags (primary container) and then in the mason jar (secondary container). However, these LDPE bags were chosen to store these mimics due to the fact it was permeable, and thus volatiles can permeate the bag and can be sniffed by a canine for training, or in this case, sampled directly into the DART stream. Therefore, following the sampling of the secondary container in these tests, the LDPE bags containing the mimics were sampled for two purposes: (1) it serves as a positive control since these mimics were made in house and their contents are known, and (2) it would confirm that these bags are in fact permeable.

The results observed indicated that the likelihood of cross-contamination are low to non-existent. Figure 123 shows the results for the sampling of smokeless powder \#1 COMPS. The lower RIC shows the instances during the sampling where 1,3-diethyl-1,3diphenylurea (methyl centralite) was detected. In this case, it is only detected when the contents of the jar, the LDPE bag containing the mimic, were sampled at the end.

The complicated part of this procedure was attempting to keep the LDPE bags close enough to sample but not close enough to the DART stream where it would melt the bag and expose its content. This became an issue in one instance which could have easily led the user to believe that the chances of cross-contamination were greater than reality. Figure 124 shows the results for the first trial of smokeless powder \#2 secondary container test. As can be seen, the target analyte was not detected or observed throughout the five-minute run, however, when the LDPE bags containing diphenyl amine were sampled, it was 
detected and was overwhelming in its abundance. Then during the second trial of this same COMPS sample, diphenyl amine was observed throughout the entire run (Figure 125).

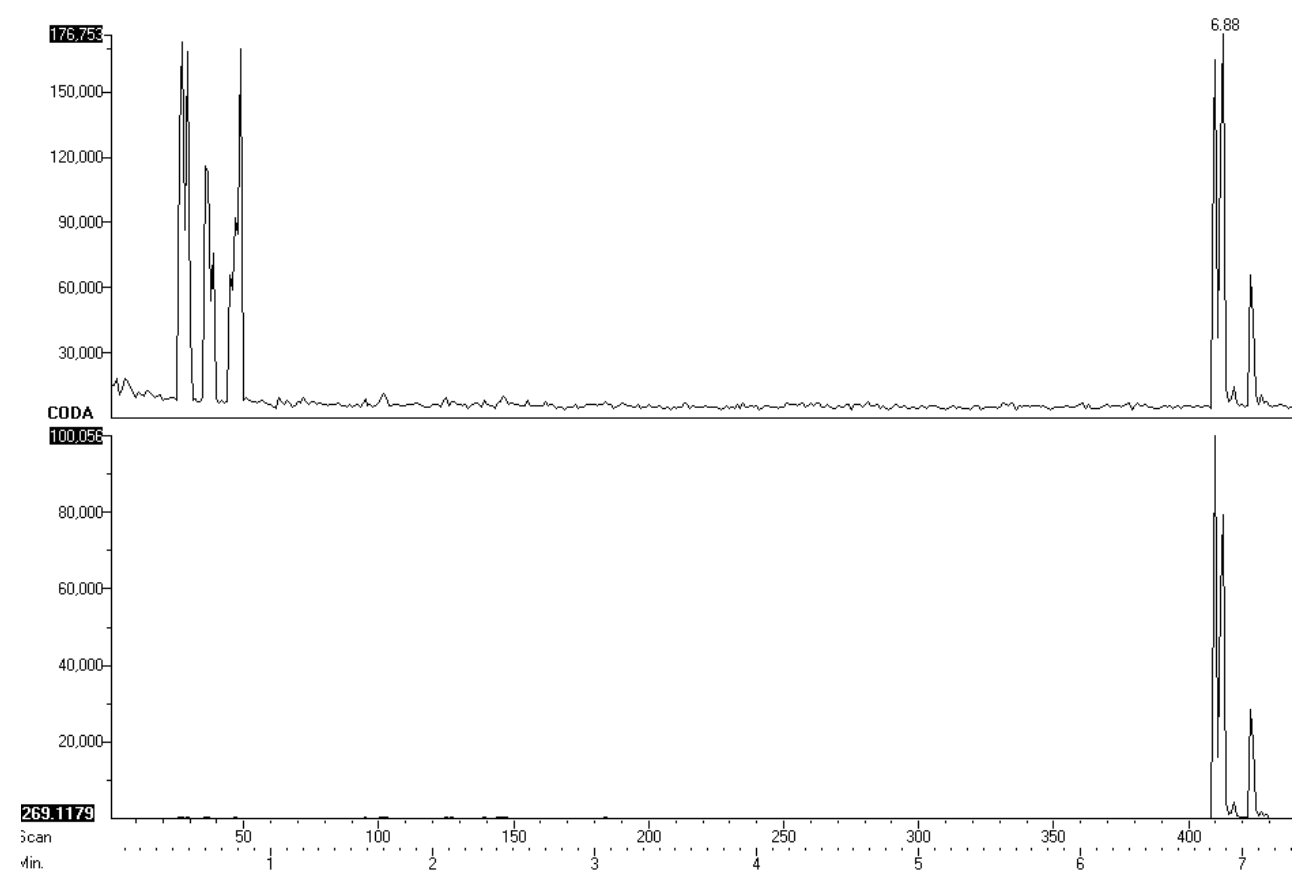

Figure 123 - TOP: RIC for smokeless powder \#1 COMPS secondary container test (first trial). First series of peaks are PEG calibrant peaks. Last series of peaks are sampling the LDPE bag containing methyl centralite standard. Bottom: RIC for $\mathrm{m} / \mathrm{z} 269$ that shows it was detected at various points during the run.

Upon further investigation, it was determined that the LDPE bag used in the first trial was held too close to the DART stream and, thus, led to the exposure of the mimic by the bag melting. While that bag was put back into the mason jar, the contents appeared to have fallen out of the bag and thus contaminated the exterior of the jar. Therefore, the appearance of cross-contamination during the second trial was a result of human error and was repeated. While the overall results will conclude that cross-contamination was not detected, it is imperative to explain why one trial did in fact show signs of crosscontamination. 


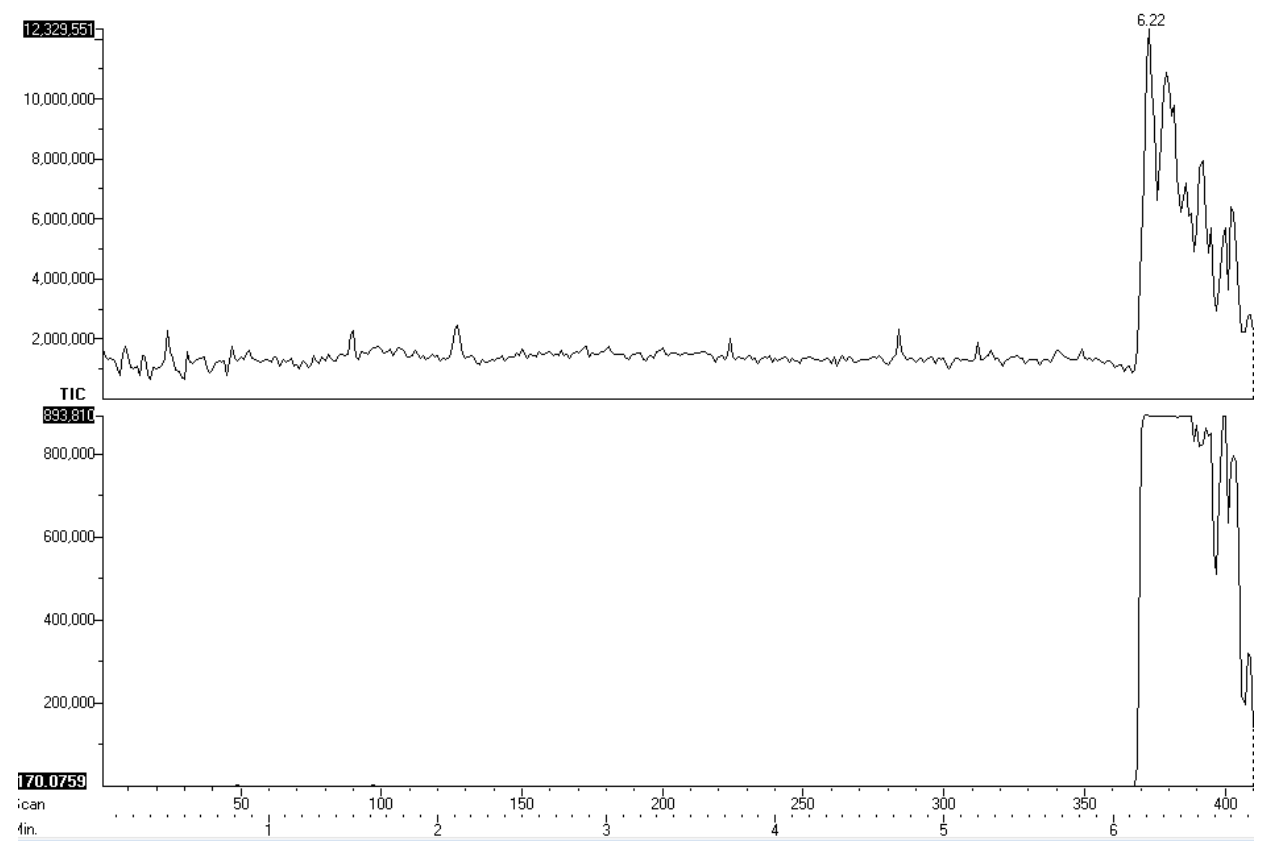

Figure 124 - TOP: RIC for smokeless powder \#2 COMPS secondary container test (first trial). First series of peaks are PEG calibrant peaks. Last series of peaks are sampling the LDPE bags containing diphenyl amine standard. Bottom: RIC for $\mathrm{m} / \mathrm{z} 170$ that shows it was detected at various points during the run.

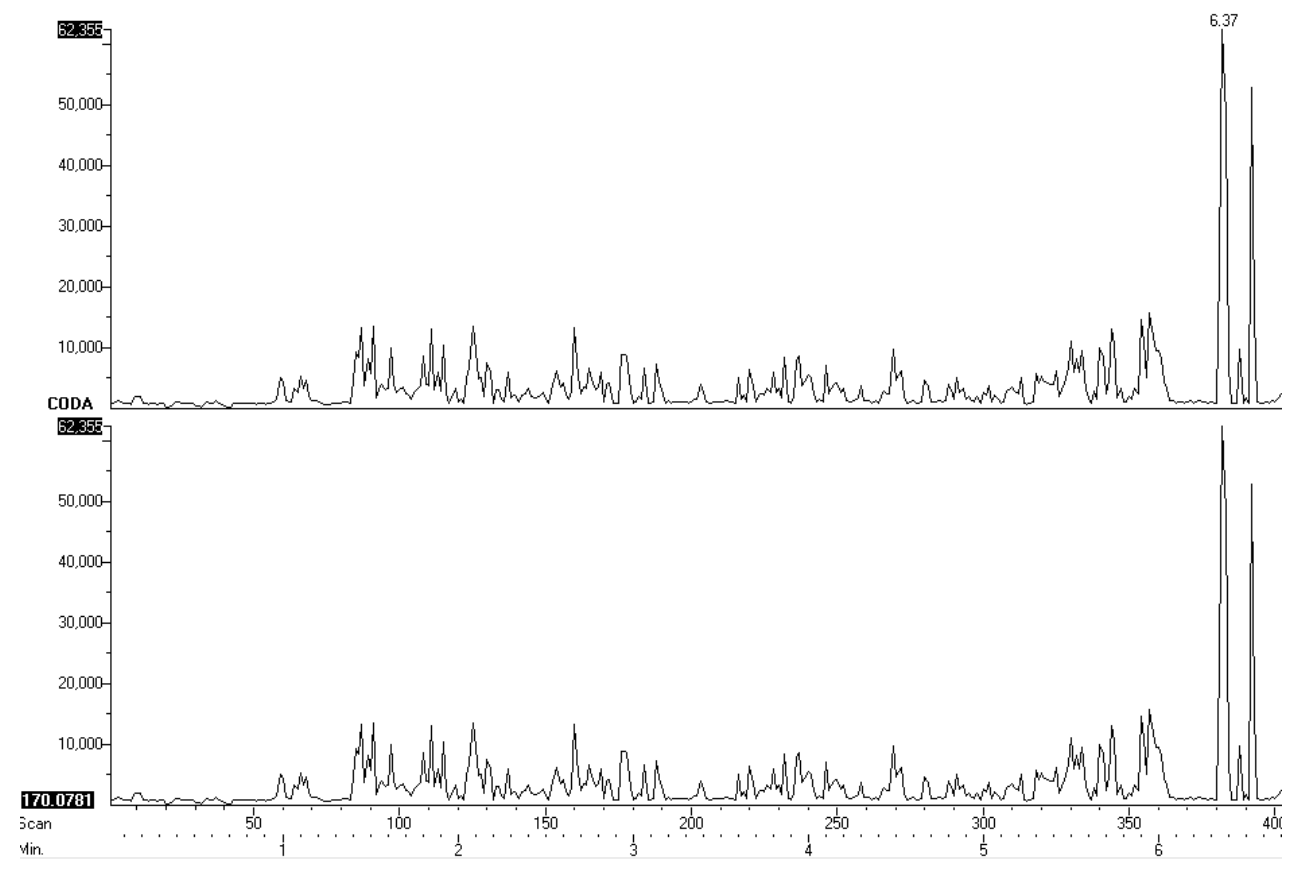

Figure 125 - TOP: RIC for smokeless powder \#2 COMPS secondary container test (second trial). First series of peaks are PEG calibrant peaks. Last series of peaks are sampling the LDPE bags containing diphenyl amine standard. Bottom: RIC for $\mathrm{m} / \mathrm{z} 170$ that shows it was detected at various points during the run. 
Although the main odorant for TNT is 2,4-dinitrotoluene, which would have to be tested in negative ion mode, the mimic is made using a smokeless powder which contains 2,4-dinitrotoluene as one of its ingredients. It also includes diphenyl amine as an ingredient and it could therefore be tested for determining the possibility of cross contamination. During all three trials, no evidence of cross-contamination was observed. Similarly, the nitroglycerin mimic is made using a smokeless powder from a different manufacturer and therefore a different mixture containing nitroglycerin as well as diphenyl amine. As is the case with 2,4-dinitrotoluene, nitroglycerin can only be analyzed using negative ion mode and therefore diphenyl amine was once again used as the identifying analyte for this experiment.

Finally, the plasticized COMPS secondary container did not produce any results that would support the idea that cross-contamination was possible. Its mimic is made up of 2-ethyl-1-hexanol which was identified when the LDPE bags were sampled in all three trials. All results for the explosive COMPS cross-contamination study is shown in Table 33.

Table 33 - Results for explosive COMPS secondary container cross-contamination tests.

$$
\text { ( } x=\text { not detected, } \checkmark=\text { detected })
$$

\begin{tabular}{|c|c|c|c|}
\multicolumn{2}{|c}{ COMP } & \multicolumn{3}{c|}{ Trial \# } \\
\hline Smokeless Powder \#1 & $\mathbf{x}$ & $\mathbf{x}$ & $\mathbf{x}$ \\
\hline Smokeless Powder \#2 & $\mathbf{x}$ & $\mathbf{x}$ & $\mathbf{x}$ \\
\hline Plasticized & $\mathbf{x}$ & $\mathbf{x}$ & $\mathbf{x}$ \\
\hline TNT & $\mathbf{x}$ & $\mathbf{x}$ & $\mathbf{x}$ \\
\hline Nitroglycerin & $\mathbf{x}$ & $\mathbf{x}$ & $\mathbf{x}$ \\
\hline
\end{tabular}




\subsection{Task 5: Survey of Narcotic and Explosive Detecting Canine Handlers}

The fifth and final task involved a survey that was sent to canine handlers that train their canines to detect narcotics or explosives. Local and state agencies in Florida and Virginia were reached out to, as well as the following associations:

- American Scent Dog Association

- American Working Dog

- National Narcotic Detector Dog Association

- World Detector Dog Organization

- Pacific Northwest Police Detection Dog Association

- United States Police Canine Association

- North American Police Work Dog Association

The main purpose behind this survey was to try and determine their thoughts regarding residual odors and the transport of these odorants when training or certifying their canines. In addition, this survey attempts to seek suggestions to help create or revisit guidelines. Another reason this survey was made was a result of the respondents not being comfortable having their responses be on the record and attributable to them personally or for the agency they work with. Therefore the answers are anonymous and the only objective response they would answer was the first question, which asks what type of agency they worked for. Overall, 32 individuals have responded to the survey. However, not all questions were answered by these individuals, therefore some questions will have less than 32 answers. 
1. Do you work for a County, State or Federal Agency?

Overall, this question received 29 of the possible 32 responses. As seen on Figure 126, two thirds of the responders worked for either county or city agencies. Three responders $(9.1 \%)$ answered that they would rather not disclose. That is in addition to the three responders who did not answer the question. As gathered from previous communications with handlers, those that worked for federal agencies were the least likely to mention or state on the record that they did in fact work for a federal agency. Only two $(6.1 \%)$ of the respondents chose 'Federal' as a response. While 'Private' agencies was not an option in the survey, $4(12.1 \%)$ respondents did select 'Other' and wrote that they worked for a private company.

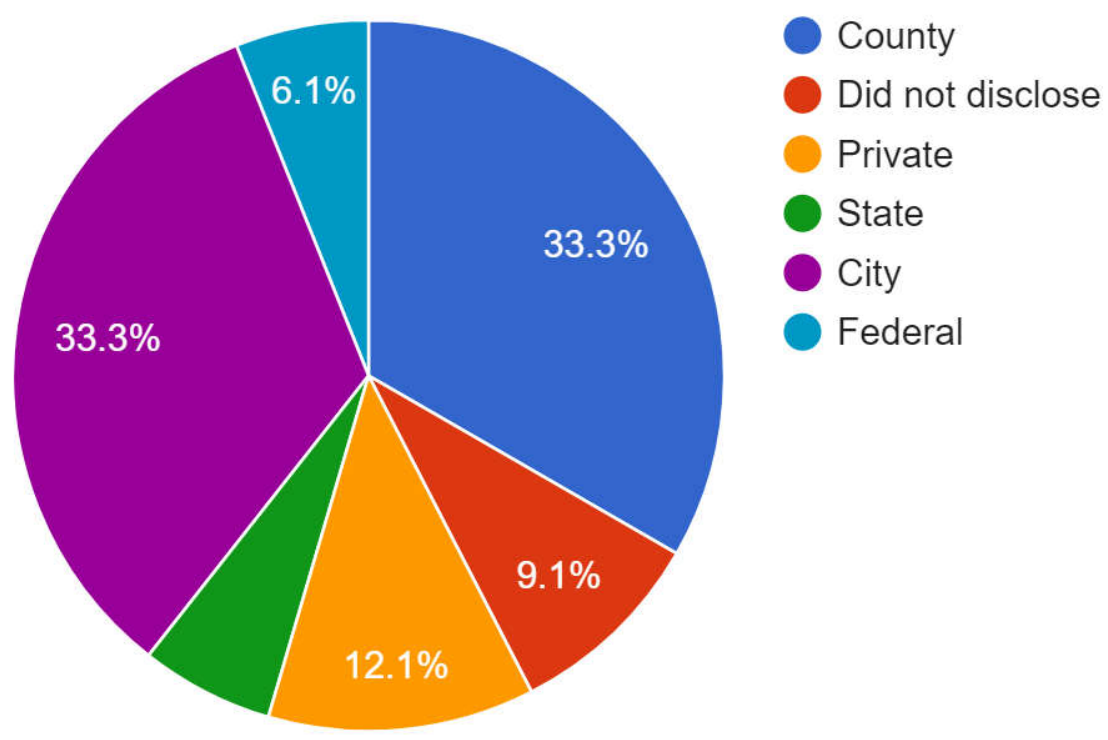

Figure 126 - 1. Do you work for a County, State or Federal Agency?

2. Do you use commercially available training aids, in-house prepared pseudo scent training aids, or actual drugs/explosives to train your canine teams? 
This question produced the most surprising responses since the overwhelming majority $(93.5 \%$, Figure 127) indicated that they use real drugs or explosives to train their canines. Of the 32 individuals who partook in this survey, only one did not answer this question. Another interesting aspect of this answer is that not a single handler from this survey used commercially available training aids to train their canines. However, a few of the responses from question \#3 suggests that they may consider IFRI training aids as inhouse prepared pseudo scent training aids and not a commercially available product.

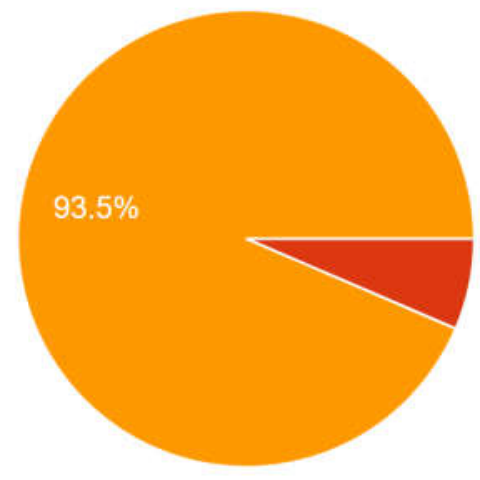

Commercially available training aids

In-house prepared pseudo scent training aids

Actual drug/explosive

Figure 127 - 2. Do you use commercially available training aids, in-house prepared pseudo scent training aids, or actual drugs/explosives to train your canine teams?

3. In your opinion, does one type or brand of training aid work better than any other? Please briefly explain your response.

This question required a short answer to be typed out, and there were 25 total responses. Almost half of these responses stated in one way or the other, that they believe using the actual controlled substance or explosive is optimal, and other training aids do not compare in terms of efficacy. However, 4 (16\%) of the respondents believe that the training aids they obtained from IFRI COMPS work the best and were more reliable. From the 
responses, it is clear that there is a huge divide in opinion with respect to what the handlers believe works best. There was also an indication in a few of these responses that they refuse to train canines using pseudo scents simply because it would need to be disclosed in court.

4. Have you observed unconfirmed alerts that can be attributed to the close proximity of targets to illicit substances?

All 32 respondents answered this question and the vast majority believe that unconfirmed alerts are $(62.5 \%)$ or could $(9.4 \%)$ be attributed to the close proximity of the target to an illicit substance. Nine (28.1\%) are confident that the distance of the target from the illicit substance would not result in an unconfirmed alert.

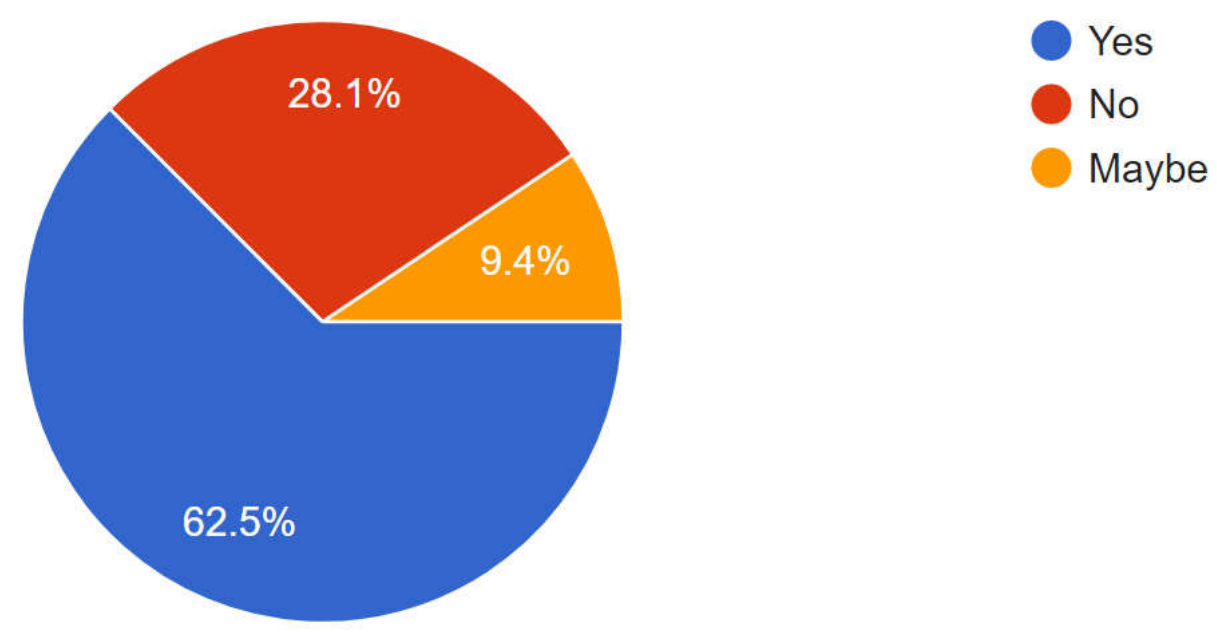

Figure 128 - 4. Have you observed unconfirmed alerts that can be attributed to the close proximity of targets to illicit substances? 
5. Have you observed unconfirmed alerts that can be attributed to potential storage issues?

Less than half $(46.9 \%)$ of the 32 respondents to this questions believe an unconfirmed alert would be a result of a potential storage problem. Whereas $18.8 \%$ answered "Maybe", and approximately a third of the respondents do not believe potential storage issues could result in an unconfirmed alert.

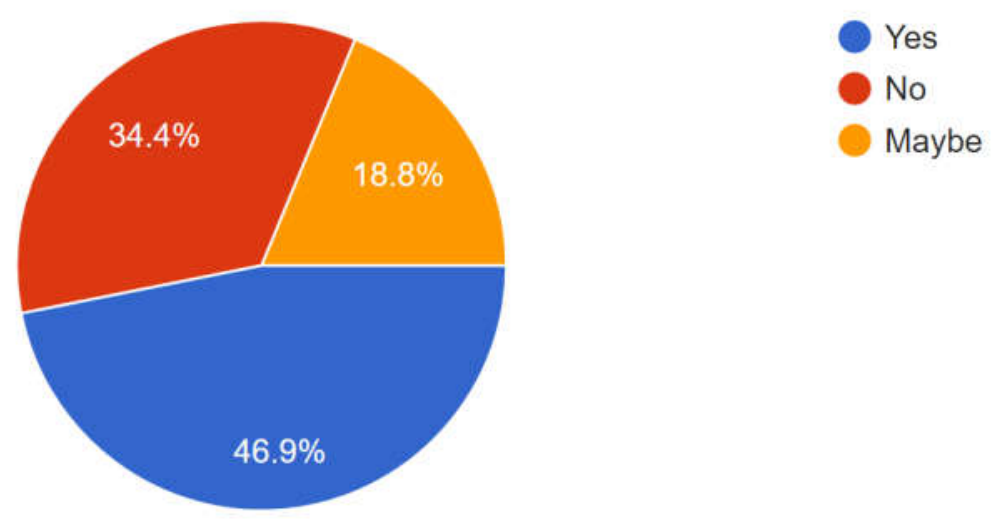

Figure $129-5$. Have you observed unconfirmed alerts that can be attributed to potential storage issues?

6. Do you believe unconfirmed alerts can be attributed to an illicit substance recently being present in that particular location?

This question explores the possibility of residual odors being the underlying issue regarding an unconfirmed alert. From the 32 responses, $71.9 \%$ of the respondents believe that this concept can be attributed to the concept of residual odor, while $15.6 \%$ answered "maybe". Only $12.5 \%$ of the respondents believe that residual odors would not be a factor in an unconfirmed alert. 


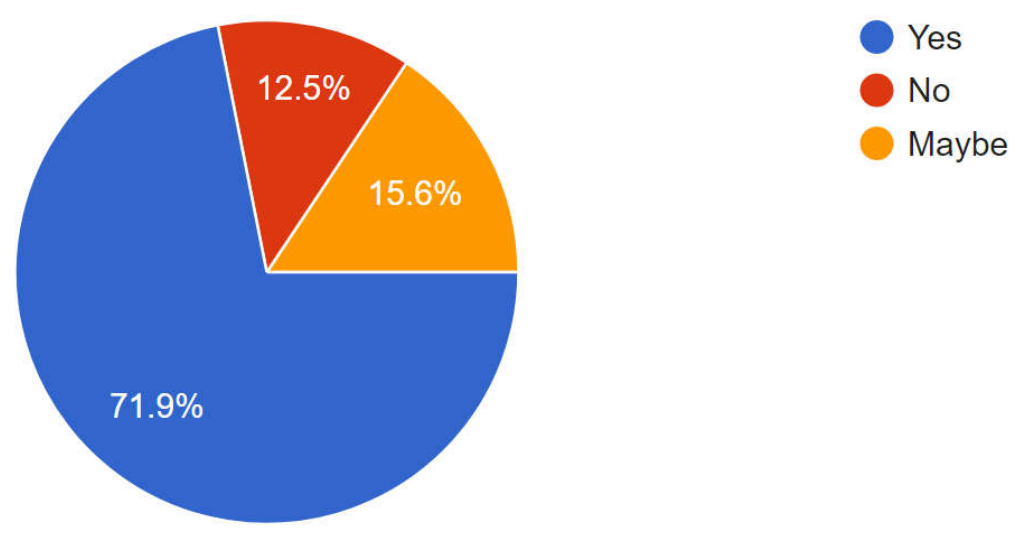

Figure 130 - 6. Do you believe unconfirmed alerts can be attributed to an illicit substance recently being present in that particular location?

7. Would you be willing to participate in a scientific study testing new training aids for effectiveness?

Three fourths of the total (32) responses specify that they would be willing to participate in a scientific study that would test new training aids for effectiveness. (Figure 131)

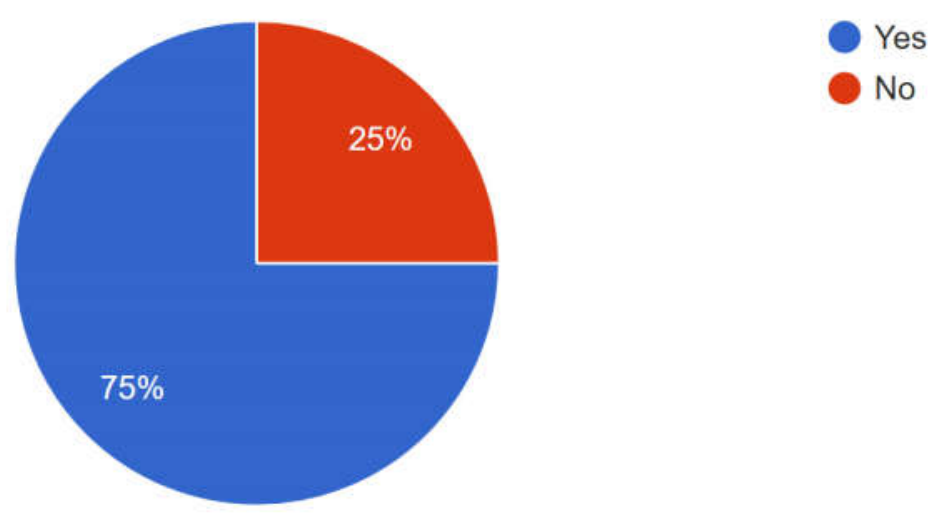

Figure 131 - 7. Would you be willing to participate in a scientific study testing new training aids for effectiveness? 
8. How are the training aids contained or stored between training?

All 32 respondents answered this question, and of them, approximately threefourths of them answered that they store the training aids in glass jars (Figure 132), and $9.1 \%$ in metal cans and only $6.3 \%$ in plastic jars. Of the $9.1 \%$ "other" responses, one respondent specified that they store the training aids in arson bags, but did not indicate the material type. Another respondent wrote that they place the training aids in "airtight cases specific to target odor".

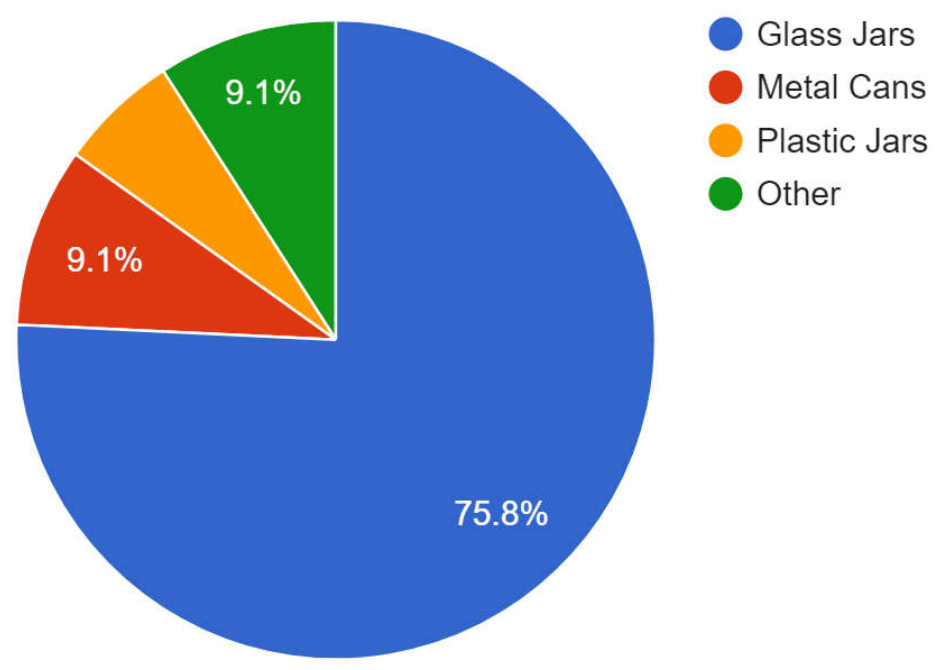

Figure $132-8$. How are the training aids contained or stored between training? 9. What is the shelf life of the training aids used to train the canines?

This question required a short answer in which 30 of the possible respondents had answered. $19.4 \%$ of the responses answered either 2 years, 2-3 years or a variation mentioning 2 years. Whereas almost a third (32.3\%) answered that the shelf-life of these training aids was just 1 year. There was one response that answered 6-12 months and 6.5\% of the trainers answered "indefinitely". 
10. How often are your training aids changed to new aids?

Almost half $(45.2 \%)$ of the 31 responses to this question answered that they change their training aids yearly, whereas $29 \%$ of them said they change it every two years. Four $(12.9 \%)$ of the responders that indicated "Other" specified that they change the training aids "when qualified dog doesn't hit on target odor", "when new narcotics are available" or "at the handler's discussion, unless cross contaminated".

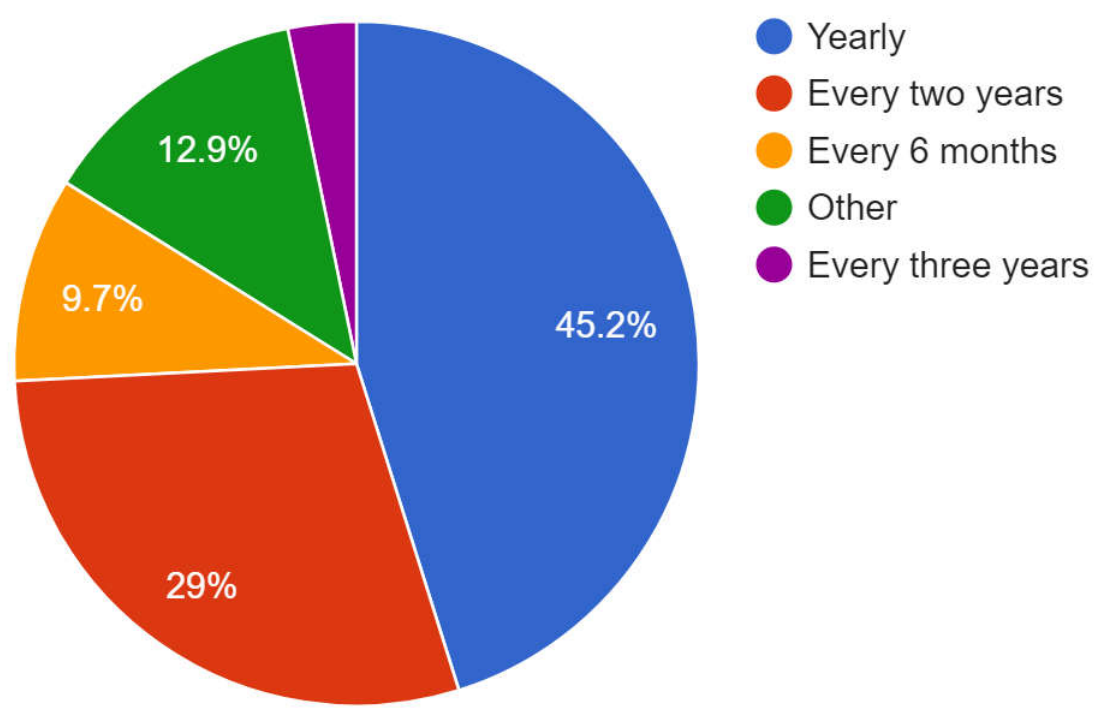

Figure 133 - 10. How often are your training aids changed to new aids?

11. What alert percentage do you think is appropriate to certify a canine for the detection of illicit substances?

The clear majority (62.5\%) suggest than an alert percentage of above $90 \%$ is appropriate. Close to one-fifth (18.8\%) think that an alert percentage above $75 \%$ is enough to certify a canine. Surprisingly, $9.4 \%$ of the 32 respondents believe that a $100 \%$ alert rate 
should be the minimum for certification. Of the $6.3 \%$ who chose "Other", one respondent did not understand what the concept of an alert percentage was, while the other says that it should be $100 \%$ in a perfectly sterile environment, but since that it is impossible in their view, they think an alert percentage of $75 \%$ was appropriate.

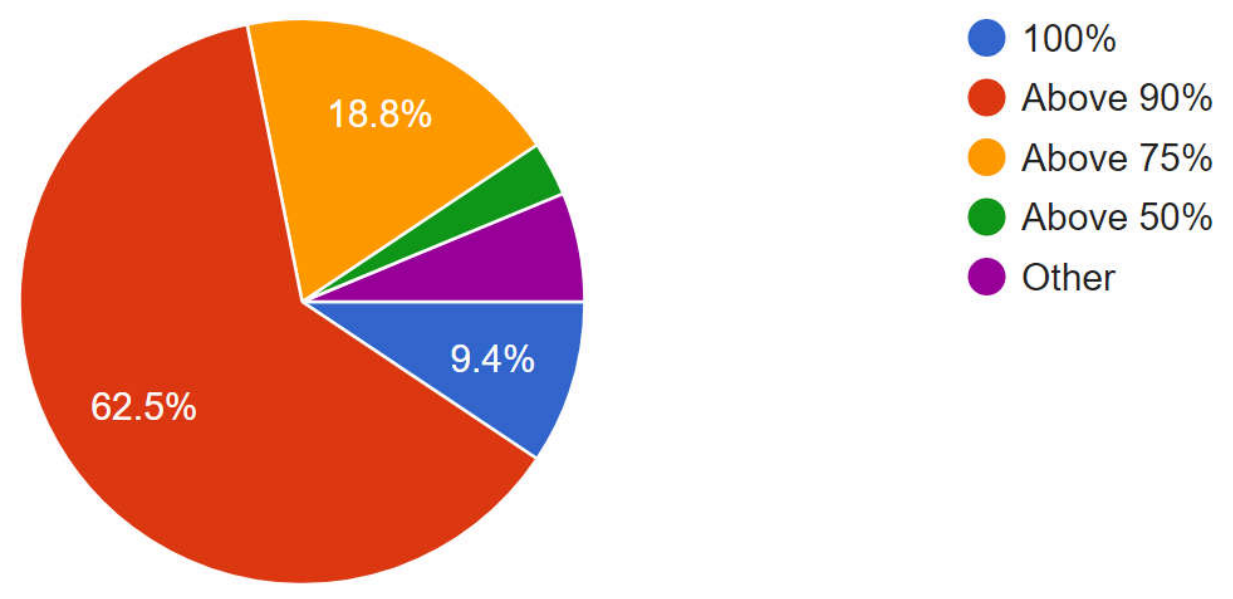

Figure 134 - 11. What alert percentage do you think is appropriate to certify a canine for the detection of illicit substances?

12. How often do you train the canines?

Over half $(53.1 \%)$ of the 32 respondents train their canines weekly, whereas approximately a third (34.4\%) train them daily. The one respondent that chose "Other" indicated that they train the canine with odors on a weekly basis but cross trains them on a daily basis. 


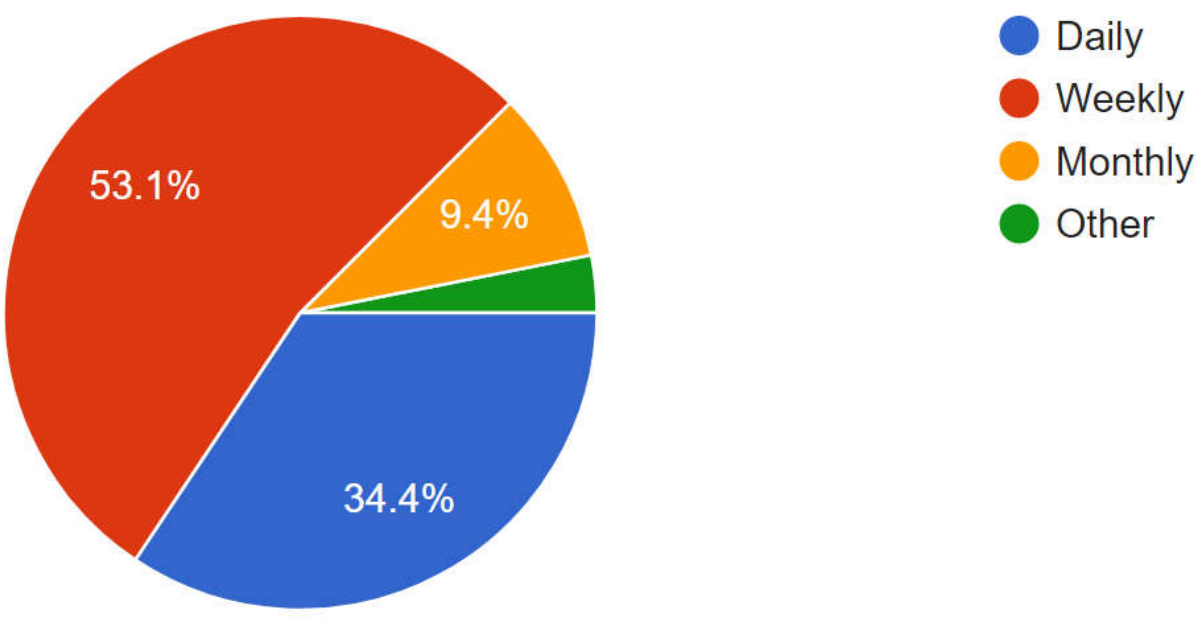

Figure 135 - 12. How often do you train the canines?

13. Are you familiar with SWGDOG?

A little under half (Figure 136) of the respondents answered that they are familiar with SWGDOG.

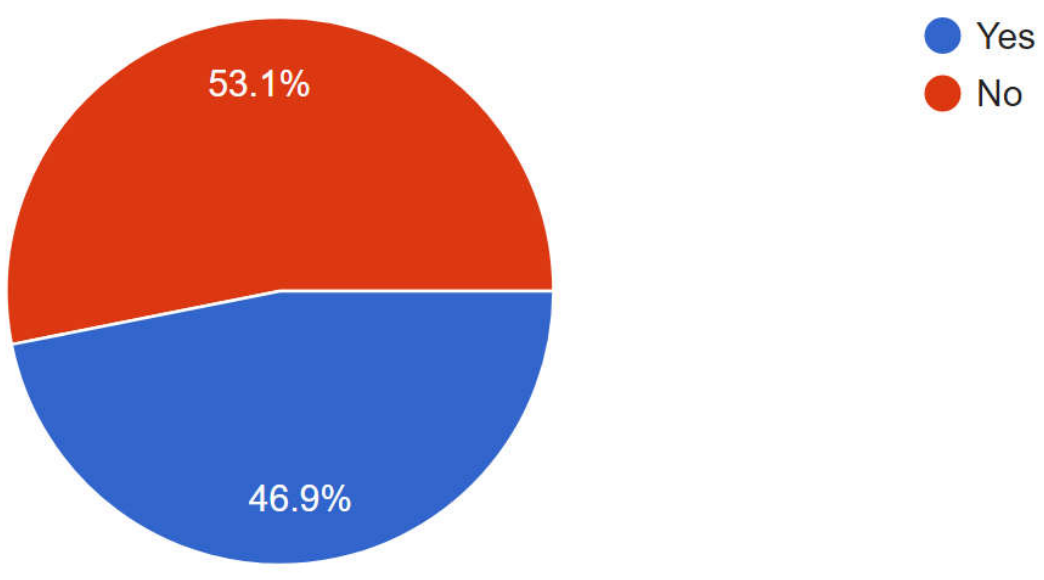

Figure 136 - 13. Are you familiar with SWGDOG? 
14. Are you familiar with OSAC?

Only $19.4 \%$ of the respondents said they were familiar with Organization of Scientific Area Committees (OSAC).

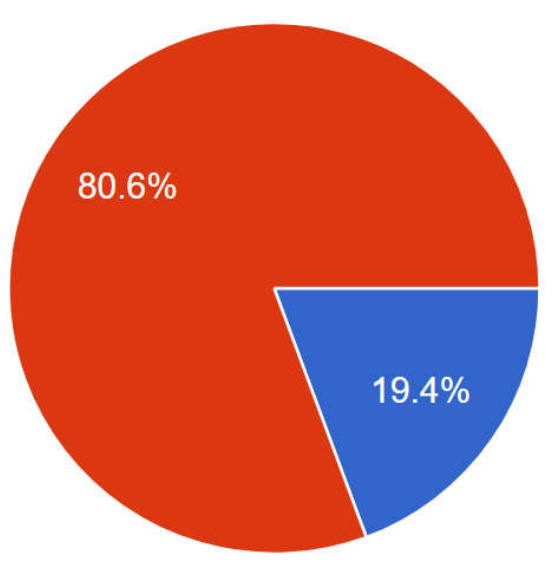

Yes

Figure 137 - 14. Are you familiar with OSAC?

15. Are your canine teams certified and under what standard?

31 of the 32 possible respondents indicated that they were certified by at least one certification agency. Over half (58.1\%) of the handlers who responded to the survey answered that they are certified by the United States Police Canine Association (USPCA). Of those, two handlers indicated they are certified by multiple agencies, including International Police Word Dog Association (IPWDA), National Association for Search and Rescue (NASAR), United Schutzhund Clubs of America (USCA), International Rescue Dog Organisation (IRO), National Narcotic Detector Dog Association (NNDDA), American Working Dogs (AWD) and Drugbeat K-9 certifications. Almost one fifth of the 
handlers certify their canines with IFRI. A few indicated they are certified by national or state standards, but did not specify which certifying agency.

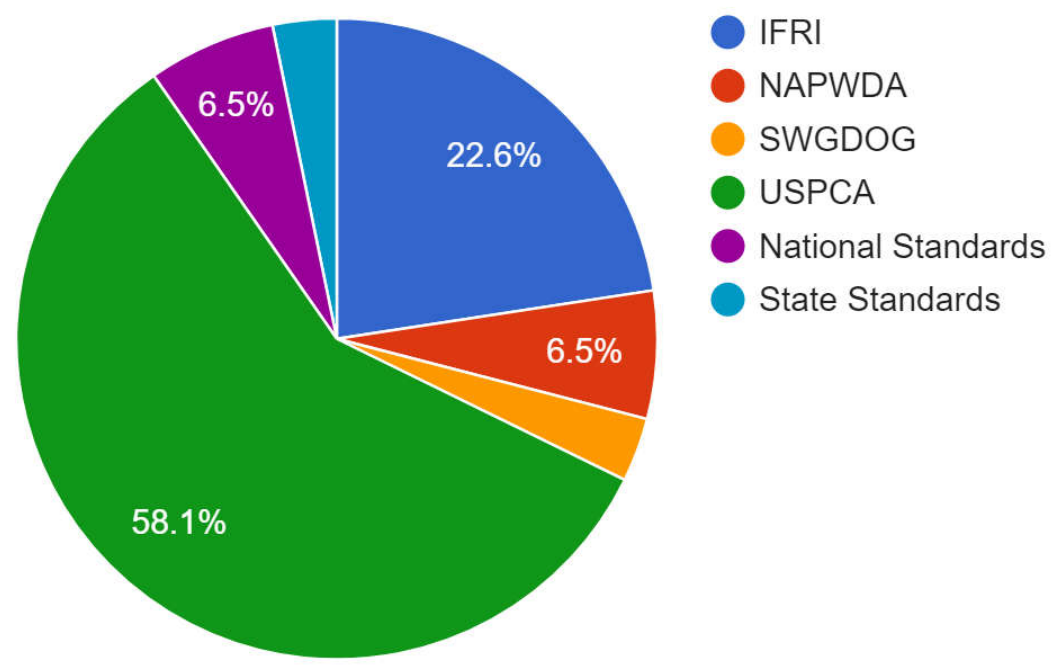

Figure 138 - 15. Are your canine teams certified and under what standard?

16. Are there any suggestions you would like SWGDOG or OSAC to consider?

Only seven of the respondents left suggestions to consider, although only two of them could be considered suggestions, as opposed to the remaining statements. One of the handlers suggested mandatory documented training on a weekly basis under the supervision of a certified trainer. Another handler thinks there needs to be a national set of compliance and standards. Two of the statements had issues with the term "unconfirmed alert", as they don't believe that such a phenomenon is possible. One handler made a note of human error playing a factor in a canine's alert rate, arguing that test conditions are never $100 \%$ perfect, accurate or clean, so therefore expecting a canine to get it right close to $100 \%$ of the time is unfair to the canine. 


\section{CONCLUSION}

The major goal of this study was to try and determine the possibility that unconfirmed alerts made by canines during their training and certifications could be a result of their close proximity to illicit substances in the vicinity or even due to the residual odor of said illicit substance in that particular area. To begin with these experiments, two narcotic odorants and two explosive odorants were chosen and were to be used for the remainder of the research. The odorants that were chosen were methyl benzoate (cocaine), benzaldehyde (methamphetamine), 2-ethyl-1-hexanol (C-4) and diethyl phthalate (smokeless powders).

Direct Analysis in Real Time (DART) coupled to an Accurate Time-Of-Flight (AccuTOFTM) mass spectrometer was chosen for its ability to detect samples in real time, without the need for the extra step of pre-concentrating or preparing a sample. Because of the various ionization energies and boiling points of these samples, one set of parameters was to be established for use during the remainder of this project. Besides the manufacturer recommended settings, the two variable parameters identified to produce significantly different results were the temperature of the gas stream and the voltage of orifice 1 . The optimum temperature was determined to be $400^{\circ} \mathrm{C}$, as it was the temperature that produced the best results for all four standard samples. The optimal orifice 1 voltage was determined to be $30 \mathrm{~V}$, as it allowed for a minimal amount of chemical induced dissociation.

The distance study (Task 2) supported the hypothesis that these odorants do not need a lot of time to travel from one area to another via the process of advection. With methyl benzoate needing approximately 23 seconds to travel a distance of $1.5 \mathrm{~m}$, 
benzaldehyde needing approximately 30 seconds to travel the same distance, and approximately 74 seconds for diethyl phthalate. Unfortunately, 2-ethyl-1-hexanol did not produce any significant results during this particular task. It simply could be due to 2-ethyl1-hexanol not being as volatile as the other samples, in that its volatile compounds prefer lingering around the substance instead of moving via advection. These results are based on what the instrument could detect, which is not as sensitive to these odors as a canine's nose. Therefore it is important to look at the values calculated and obtained in this task as close to an upper limit, since canines could probably detect them sooner depending on the concentration.

The third task attempted to answer the questions involving the possibility of residual odor playing a factor in unconfirmed alerts. This was problematic since no observable results were identified to help lend credence to this phenomenon for exposure times at or below one hour. While the instrument has its advantages, it is not without its limitations. It could be entirely possible that the residual odors that 'stuck' on the various substrates during their exposure times dissipated when moving said substrate from the plastic container to the DART stream, or could have even been 'knocked off' of the substrate from modest aerodynamics. It is also possible that the amount of odor on the substrate was not high enough to be detectable by the instrument, but high enough to be detectable by the canine. Because of these results, some ideas for future work will be discussed in the next section.

This research also considered the possibility of cross-contamination between the training aids made by IFRI. Had cross-contamination been observed, then that would lead 
to having the packaging and storage conditions changed. However, no evidence of crosscontamination was observed in either the narcotic COMPS, which use foil ziplock bags to store the mimics in, nor were they observed in the explosive COMPS that are stored in glass mason jars.

From the survey conducted, it was surprising to see that $93.5 \%$ of the handlers use real drugs or explosives to train their canines, as opposed to using commercially available or in-house prepared pseudo scent aids. Almost two-thirds of handlers believed unconfirmed alerts can be attributed to the close proximity of targets to illicit substances, while almost three-fourths of them indicated they believed residual odor did or could play a role in unconfirmed alerts. A little less than half were familiar with SWGDOG while just one in five were familiar with OSAC.

In conclusion, the results of this study indicate that the close proximity of an illicit substance to a target could very well lead to an unconfirmed alert by a canine during their training and certifications. Through no fault of their own, this could potentially lead to the canines failing their certifications. Therefore, a change in guidelines may be necessary to prevent this possibility. One way this could be done is to increase the distance between the boxes or objects used during certifications, and when the opportunity permits, different rooms would ideally solve this problem. Although a specific distance is not mentioned in the guidelines, a minimum distance should be considered. Since it takes less than a minute for a detectable amount of both methyl benzoate and benzaldehyde to transport from a location 3 meters away, the further the boxes used in these certifications are from one another the better. While the outdoors might seem preferable because of the space, it is 
important to conduct these tests in a more controlled atmosphere. Although it resembles scenarios a canine would encounter on duty, it is important for the certifications to be conducted in a way that does not penalize the canine for flaws in the certification set up. Therefore, guidelines should explicitly attempt to eliminate or substantially reduce potentials for unconfirmed alerts by suggesting a minimum distance between target areas. Another idea involves using a minimal amount of the target odor or substance, to reduce the amount of volatile organic compounds traveling within an odor plume to different areas.

Based on the results of this research, to prevent the possibility of residual odors being a factor in unconfirmed alerts, the target drug or explosive should be placed in the box or object right before a run, and removed immediately after the fact. Although no data showed the odors persisting onto the various substrates at or below one hour exposure times, this topic and the questions revolving residual odors should be researched further. With respect to the substrate types, there is a clear preference in what type should be used as it does not absorb odors like the remaining two. Methyl benzoate did not produce any results with metal, while benzaldehyde and 2-ethyl-1-hexanol only produced results after 24-hour exposure times. Diethyl phthalate was the only odorant consistently detected by the instrument at exposure times ranging from 2-24 hours. When looking at the data collectively, it is clear that metal is an ideal substrate to use for enclosures or boxes used during certifications. 


\section{FUTURE WORK}

While considering the limitations of the DART, a few ideas that require extra steps could help produce results that could help support or reject a hypothesis; the idea that residual odors may play a factor in unconfirmed alerts. First, to prevent the possibility of the odorants dissipating or being 'knocked off' of the substrate by air, the substrates should be placed in vials immediately within the plastic containers, and capped off with a top that has a septum. This way, it can be pre-concentrated on a SPME fiber and then tested using GC-MS.

The other project would involve simply testing this hypothesis during canine trainings. An illicit substance should be placed in an area for a certain amount of time and then removed, and the canine should then sniff that area at various time intervals to see if they alert or not. The area should be secluded and not open to an area where regular training occurs. It would be beneficial if the canine were to test the area prior to placing the illicit substances there so that it serves as a blank sample test, to ensure nothing was present previously. 


\section{REFERENCES}

[1] A. M. Curran, P. A. Prada, and K. G. Furton, "Canine human scent identifications with post-blast debris collected from improvised explosive devices," Forensic Sci. Int., vol. 199, no. 1-3, pp. 103-108, 2010.

[2] N. Lorenzo et al., "Laboratory and field experiments used to identify Canis lupus var. familiaris active odor signature chemicals from drugs, explosives, and humans," Anal. Bioanal. Chem., vol. 376, no. 8, pp. 1212-1224, Aug. 2003.

[3] J. M. Perr, K. G. Furton, and J. R. Almirall, "Application of a SPME-IMS detection system for explosives detection," in Proceedings of SPIE - The International Society for Optical Engineering, 2005, vol. 5778, no. PART II, pp. 667-672.

[4] B. J. Buchanan, Gunpowder, explosives and the state: a technological history. Ashgate, 2006.

[5] A. Beveridge, Forensic investigation of explosions. CRC Press, 2012.

[6] P. W. Cooper and S. R. Kurowski, Introduction to the technology of explosives. VCH, 1996.

[7] R. O. Keto, "Improved Method for the Analysis of the Military Explosive Composition C-4,” J. Forensic Sci. JFSCA, vol. 31, no. 1, pp. 241-249, 1986.

[8] S. Bell, Forensic chemistry. Pearson Prentice Hall, 2006.

[9] I. Glassman, R. A. Yetter, I. Glassman, and R. A. Yetter, "Chapter 5 - Detonation," in Combustion, 2008, pp. 261-309.

[10] Technical/Scientific Working Group for Fire and Explosions Analysis (T/SWGFEX), "TWGFEX Laboratory Explosion Group Standards \& Protocols Committee." .

[11] Virginia Department of Forensic Science, "Trace Evidence Procedures Manual," 2016.

[12] C. $>$. Amera-Chem Inc. $<$ Grand Junction, Drug Identification Bible. Amera-Chem, 2004.

[13] "Controlled Substances -Alphabetical Order - DEA CSA SUBSTANCE NUMBER SCH NARC OTHER NAMES."

[14] "DEA - Federal Trafficking Penalties." [Online]. Available: https://www.dea.gov/druginfo/ftp3.shtml. [Accessed: 07-Mar-2017].

[15] "DEA.gov / Statistics \&amp; Facts." [Online]. Available: 
https://www.dea.gov/resource-center/statistics.shtml. [Accessed: 27-Feb-2017].

[16] Virginia Department of Forensic Science, "Controlled Substances Procedures Manual," 2016.

[17] "SCIENTIFIC WORKING GROUP FOR THE ANALYSIS OF SEIZED DRUGS (SWGDRUG) RECOMMENDATIONS."

[18] C. L. O'Neal, D. J. Crouch, and A. A. Fatah, "Validation of twelve chemical spot tests for the detection of drugs of abuse.," Forensic Sci. Int., vol. 109, no. 3, pp. 189 201, Apr. 2000.

[19] E. Stahl, Ed., Thin-Layer Chromatography. Berlin, Heidelberg: Springer Berlin Heidelberg, 1969.

[20] S. E. Howlett and R. R. Steiner, "Validation of Thin Layer Chromatography with AccuTOF-DART??? Detection for Forensic Drug Analysis," J. Forensic Sci., vol. 56, no. 5, pp. 1261-1267, 2011.

[21] R. J. Harper, J. R. Almirall, and K. G. Furton, "Identification of dominant odor chemicals emanating from explosives for use in developing optimal training aid combinations and mimics for canine detection," Talanta, vol. 67, no. 2, pp. 313$327,2005$.

[22] K. G. Furton, N. I. Caraballo, M. M. Cerreta, and H. K. Holness, "Advances in the use of odour as forensic evidence through optimizing and standardizing instruments and canines.," Philos. Trans. R. Soc. Lond. B. Biol. Sci., vol. 370, no. 1674, Aug. 2015.

[23] L. Fernando Mendez Pardo, A. M. Perez-Acosta, M. Pardo, and L. Fernando, "Research in Colombia on Explosives Detection by Rats Recommended Citation," J. ERW Mine Action, vol. 13, no. 3.

[24] J. A. Shaw et al., "Polarization lidar measurements of honey bees in flight for locating land mines."

[25] J. K. Borchardt, "New weed may flag land mines," CSMonitor.com. [Online]. Available: http://www.csmonitor.com/2004/0219/p11s01-stss.html. [Accessed: 21Mar-2017].

[26] W. F. Handy, M. Harrington, and D. J. Pittman, "The K-9 Corps: The Use of Dogs in Police Work," J. Crim. Law. Criminol. Police Sci., vol. 52, no. 3, p. 328, 1961.

[27] H. C. Underhill, A treatise on the law of evidence : with a discussion of the principles and rules which govern its presentation, reception and exclusion, and the examination of witnesses in court. Chicago, T.H. Flood and Company, 1894. 
[28] "Police Canines in History - Dogs for Law Enforcement." [Online]. Available: http://www.dogsforlawenforcement.org/police-canines-in-history.html. [Accessed: 26-Mar-2017].

[29] T. Jezierski, J. Ensminger, and L. E. Papet, Canine Olfaction Science and Law: Advances in Forensic Science, Medicine, Conservation, and Environmental Remediation. .

[30] T. D. Smith, K. P. Bhatnagar, P. Tuladhar, and A. M. Burrows, "Distribution of olfactory epithelium in the primate nasal cavity: Are microsmia and macrosmia valid morphological concepts?," Anat. Rec., vol. 281 A, no. 1, pp. 1173-1181, Nov. 2004.

[31] P. Quignon et al., "Comparison of the canine and human olfactory receptor gene repertoires.," Genome Biol., vol. 4, no. 12, p. R80, 2003.

[32] B. A. Craven, E. G. Paterson, and G. S. Settles, "The fluid dynamics of canine olfaction: unique nasal airflow patterns as an explanation of macrosmia," J. R. Soc. Interface, vol. 7, no. 47, pp. 933-943, Jun. 2010.

[33] A. Rinaldi, "The scent of life. The exquisite complexity of the sense of smell in animals and humans.," EMBO Rep., vol. 8, no. 7, pp. 629-33, Jul. 2007.

[34] H. Hylton, "Dogs Sniffing Out Crime: Science or Shaky Evidence? - TIME," Time, $2009 . \quad$ [Online]. Available: http://content.time.com/time/nation/article/0,8599,1913987,00.html.

[35] J. Schwartz, "Picked From a Lineup, on a Whiff of Evidence - NYTimes.com," The New York Times, 2009. [Online]. Available: http://www.nytimes.com/2009/11/04/us/04scent.html?_r=0.

[36] The Innocence Project of Texas, "Dog Scent Lineups. A Junk Science Injustice," 2009.

[37] R. J. Harper and K. G. Furton, Counterterrorist Detection Techniques of Explosives. 2007.

[38] K. Furton, J. Greb, and H. Holness, "The Scientific Working Group on Dog and Orthogonal Detector Guidelines (SWGDOG)."

[39] Cornell Law, "Fourth Amendment | Constitution | US Law | LII / Legal Information Institute." [Online]. Available: https://www.law.cornell.edu/constitution/fourth_amendment.

[40] Nos, "Supreme Court of the United States," vol. 7, no. 9, pp. 5-11, 1953.

[41] J. E. Ferguson, "Supreme Court of Florida," vol. 3, no. 1, pp. 1-9, 2012. 
[42] SUPREME COURT OF THE UNITED STATES, Illinois v. Caballes. 2004.

[43] SUPREME COURT OF THE UNITED STATES, Florida v. Harris. 2012.

[44] K. G. Furton, Y.-C. Hong, Y.-L. Hsu, T. Luo, S. Rose, and J. Walton, "Identification of odor signature chemicals in cocaine using solid-phase microextraction-gas chromatography and detector-dog response to isolated compounds spiked on U.S. paper currency.," J. Chromatogr. Sci., vol. 40, no. 3, pp. 147-155, 2002.

[45] M. Macias, "The Development of an Optimized System of Narcotic and Explosive Contraband Mimics for Calibration and Training of Biological Detectors," FIU Electron. Theses Diss., 2009.

[46] Y.-L. Hsu, "Correlation of detector dog alerts to cocaine decomposition products found in illicit forensic specimens," ProQuest ETD Collect. FIU, 1998.

[47] N. Lorenzo et al., "Laboratory and field experiments used to identify Canis lupus var. familiaris active odor signature chemicals from drugs, explosives, and humans," Anal. Bioanal. Chem., vol. 376, no. 8, pp. 1212-1224, 2003.

[48] D.-T. T. Vu, "SPME/GC-MS Characterization of Volatiles Associated with Methamphetamine: Toward the Development of a Pseudomethamphetamine Training Material," J. Forensic Sci., vol. 46, no. 5, p. 15095J, Sep. 2001.

[49] K. G. Furton and R. J. Harper, "Controlled Odor Mimic Permeation System," US20080295783 A1, 2007.

[50] L. V Hood, M. E. Dames, and G. T. Barry, "Headspace volatiles of marijuana.," Nature, vol. 242, no. 5397, pp. 402-3, Apr. 1973.

[51] N. Lorenzo et al., "Laboratory and field experiments used to identify Canis lupus var. familiaris active odor signature chemicals from drugs, explosives, and humans," Anal. Bioanal. Chem., vol. 376, no. 8, pp. 1212-1224, Aug. 2003.

[52] R. J. Harper, "Improving the scientific reliability of biological detection of explosives by Canis familiaris: Active odour signatures and their implications," Florida Int. Univ., 2005.

[53] M. Williams et al., "Canine detection odor signatures for explosives," in SPIE 3575, Enforcement and Security Technologies, 1998, pp. 291-301.

[54] M. Joshi, K. Rigsby, and J. R. Almirall, "Analysis of the headspace composition of smokeless powders using GC-MS, GC- $\mu \mathrm{ECD}$ and ion mobility spectrometry," Forensic Sci. Int., vol. 208, no. 1-3, pp. 29-36, May 2011.

[55] R. Mistafa, K9 explosive detection: A Manual for Trainers. Detselig Enterprises, 1998. 
[56] M. E. Cablk, J. C. Sagebiel, J. S. Heaton, and C. Valentin, "Olfaction-based Detection Distance: A Quantitative Analysis of How Far Away Dogs Recognize Tortoise Odor and Follow It to Source," Sensors, vol. 8, no. 4, pp. 2208-2222, Mar. 2008.

[57] C. Lytridis, G. S. Virk, Y. Rebour, and E. E. Kadar, "Odor-Based Navigational Strategies for Mobile Agents," Adapt. Behav., vol. 9, no. 3-4, pp. 171-187, Sep. 2001.

[58] C. Lytridis, E. E. Kadar, and G. S. Virk, "A systematic approach to the problem of odour source localisation,” Auton. Robots, vol. 20, no. 3, pp. 261-276, Jun. 2006.

[59] M. E. Staymates et al., "Biomimetic Sniffing Improves the Detection Performance of a 3D Printed Nose of a Dog and a Commercial Trace Vapor Detector," Sci. Rep., vol. 6, p. 36876, Dec. 2016.

[60] B. A. Craven et al., "Reconstruction and Morphometric Analysis of the Nasal Airway of the Dog (Canis familiaris) and Implications Regarding Olfactory Airflow," Anat. Rec. Adv. Integr. Anat. Evol. Biol., vol. 290, no. 11, pp. 1325-1340, Nov. 2007.

[61] S. Fiset and V. LeBlanc, "Invisible displacement understanding in domestic dogs (Canis familiaris): the role of visual cues in search behavior," Anim. Cogn., vol. 10, no. 2, pp. 211-224, Mar. 2007.

[62] N. Uchida, A. Kepecs, and Z. F. Mainen, "Seeing at a glance, smelling in a whiff: rapid forms of perceptual decision making," Nat. Rev. Neurosci., vol. 7, no. 6, pp. 485-491, Jun. 2006.

[63] A. Kepecs, N. Uchida, and Z. F. Mainen, "The Sniff as a Unit of Olfactory Processing," Chem. Senses, vol. 31, no. 2, pp. 167-179, Dec. 2005.

[64] T. Lochmatter, X. Raemy, and A. Martinoli, "Odor Source Localization with Mobile Robots,” Bull. Swiss Soc. Autom. Control, no. 46, pp. 11-14, 2007.

[65] J. P. Crimaldi, M. A. R. Koehl, and J. R. Koseff, "Effects of the Resolution and Kinematics of Olfactory Appendages on the Interception of Chemical Signals in a Turbulent Odor Plume," Environ. Fluid Mech., vol. 2, no. 1/2, pp. 35-64, 2002.

[66] P. Moore and J. Crimaldi, "Odor landscapes and animal behavior: tracking odor plumes in different physical worlds," J. Mar. Syst., vol. 49, no. 1-4, pp. 55-64, Aug. 2004.

[67] R. B. Cody, J. A. Laramée, and H. D. Durst, "Versatile New Ion Source for the Analysis of Materials in Open Air under Ambient Conditions," Anal. Chem., vol. 77, no. 8, pp. 2297-2302, Apr. 2005. 
[68] H. H. Maurer, "Liquid chromatography-mass spectrometry in forensic and clinical toxicology," J. Chromatogr. B Biomed. Sci. Appl., vol. 713, no. 1, pp. 3-25, Aug. 1998.

[69] R. B. Cody, J. a Laramée, J. M. Nilles, and H. D. Durst, "Direct Analysis in Real Time (DART) Mass Spectrometry," JEOL news, vol. 40, no. 1, pp. 8-12, 2005.

[70] J. Tamura and J. Osuga, "New Generation LC-TOF/MS “AccuTOF," JEOL Ltd.

[71] J. M. Nilles, T. R. Connell, S. T. Stokes, and H. Dupont Durst, "Explosives Detection Using Direct Analysis in Real Time (DART) Mass Spectrometry," Propellants, Explos. Pyrotech., vol. 35, no. 5, pp. 446-451, Oct. 2010.

[72] R. W. Jones and J. F. McClelland, "Analysis of writing inks on paper using direct analysis in real time mass spectrometry," Forensic Sci. Int., vol. 231, no. 1-3, pp. 73-81, 2013.

[73] R. W. Jones, R. B. Cody, and J. F. McClelland, "Differentiating writing inks using direct analysis in real time mass spectrometry," J. Forensic Sci., vol. 51, no. 4, pp. 915-918, 2006.

[74] E. S. Chernetsova, G. E. Morlock, and I. a Revelsky, "DART mass spectrometry and its applications in chemical analysis," Russ. Chem. Rev., vol. 80, pp. 235-255, 2011.

[75] J. Hajslova, T. Cajka, and L. Vaclavik, "Challenging applications offered by direct analysis in real time (DART) in food-quality and safety analysis," TrAC - Trends in Analytical Chemistry, vol. 30, no. 2. pp. 204-218, 2011.

[76] R. R. Steiner and R. L. Larson, "Validation of the Direct Analysis in Real Time Source for Use in Forensic Drug Screening," J. Forensic Sci., vol. 54, no. 3, pp. 617-622, 2009.

[77] E. Sisco, J. Dake, and C. Bridge, "Screening for trace explosives by AccuTOF???DART??: An in-depth validation study," Forensic Sci. Int., vol. 232, no. 1-3, 2013.

[78] L. Wang, S. Zeng, T. Chen, and H. Qu, "Direct analysis in real time mass spectrometry, a process analytical technology tool for real-time process monitoring in botanical drug manufacturing," J. Pharm. Biomed. Anal., vol. 91, pp. 202-209, 2014.

[79] R. A. Musah et al., "A High Throughput Ambient Mass Spectrometric Approach to Species Identification and Classification from Chemical Fingerprint Signatures.," Sci. Rep., vol. 5, no. May, 2015.

[80] A. D. Lesiak, "Recent advances in forensic drug analysis by DART-MS," Futur. Sci., vol. 6, no. August, 2014. 
[81] T. Nah, M. Chan, S. R. Leone, and K. R. Wilson, "Real time in situ chemical characterization of submicrometer organic particles using direct analysis in real time-mass spectrometry," Anal. Chem., vol. 85, no. 4, pp. 2087-2095, 2013.

[82] K. Clemons, J. Dake, E. Sisco, and G. F. Verbeck, "Trace analysis of energetic materials via direct analyte-probed nanoextraction coupled to direct analysis in real time mass spectrometry," Forensic Sci. Int., vol. 231, no. 1-3, pp. 98-101, 2013.

[83] V. Hrbek, L. Vaclavik, O. Elich, and J. Hajslova, "Authentication of milk and milkbased foods by direct analysis in real time ionization-high resolution mass spectrometry (DART-HRMS) technique: A critical assessment," Food Control, vol. 36, no. 1, pp. 138-145, Feb. 2014.

[84] E. Jagerdeo and M. Abdel-Rehim, "Screening of Cocaine and Its Metabolites in Human Urine Samples by Direct Analysis in Real-Time Source Coupled to Timeof-Flight Mass Spectrometry After Online Preconcentration Utilizing Microextraction by Packed Sorbent," J. Am. Soc. Mass Spectrom., vol. 20, no. 5, pp. 891-899, 2009.

[85] A. Rodriguez-Lafuente, F. S. Mirnaghi, and J. Pawliszyn, "Determination of cocaine and methadone in urine samples by thin-film solid-phase microextraction and direct analysis in real time (DART) coupled with tandem mass spectrometry," Anal. Bioanal. Chem., vol. 405, no. 30, pp. 9723-9727, Dec. 2013.

[86] G. A. Harris and F. M. Fernández, "Simulations and Experimental Investigation of Atmospheric Transport in an Ambient Metastable-Induced Chemical Ionization Source," Anal. Chem., vol. 81, no. 1, pp. 322-329, Jan. 2009.

[87] "IUPAC Gold Book - collision-induced dissociation inmass spectrometry." [Online]. Available: https://goldbook.iupac.org/C01167.html. [Accessed: 08-Mar2017].

[88] D. A. Skoog, F. J. Holler, and S. R. Crouch, Principles of Instrumental Analysis Sixth Edition. 1998.

[89] "Microchannel plate detector and methods for their fabrication," 2011.

[90] “AccuTOF ${ }^{\mathrm{TM}}$-DART ${ }^{\mathrm{TM}}$ Training Guide JEOL,” JEOL Train. Guid., vol. 6043, no. 978, 2007.

[91] W. Windig and W. F. Smith, "Chemometric analysis of complex hyphenated data," J. Chromatogr. A, vol. 1158, no. 1-2, pp. 251-257, Jul. 2007.

[92] P. Mansfield, "Instantaneous Detection of Illicit Drugs on Currency." 
[93] K. G. Furton, Y. -c. Hong, Y.-L. Hsu, T. Luo, S. Rose, and J. Walton, "Identification of Odor Signature Chemicals in Cocaine Using Solid-Phase Microextraction-Gas Chromatography and Detector-Dog Response to Isolated Compounds Spiked on U.S. Paper Currency," J. Chromatogr. Sci., vol. 40, no. 3, pp. 147-155, Mar. 2002.

[94] JEOL, “AccuTOF-DART SVP Training Guide.” JEOL, USA, Inc., Peabody, MA. 


\section{APPENDICES}

DART-AccuTOF Manufacturer Training Manual.[94]

JEOL USA, Inc.

AccuTOF-DART Training Guide

\section{System Start-up/Shutdown/Idle}

Starting AccuTOF -DART TM after a Complete Shutdown

Use these procedures to power up the instrument after a power outage or after the instrument has been completely shut down.

\section{Powering up the AccuTOFTM}

a. If it is off, turn on the main breaker switch found on the back of the instrument.

b. Push the Power ON button on the front panel (VENT will be illuminated).

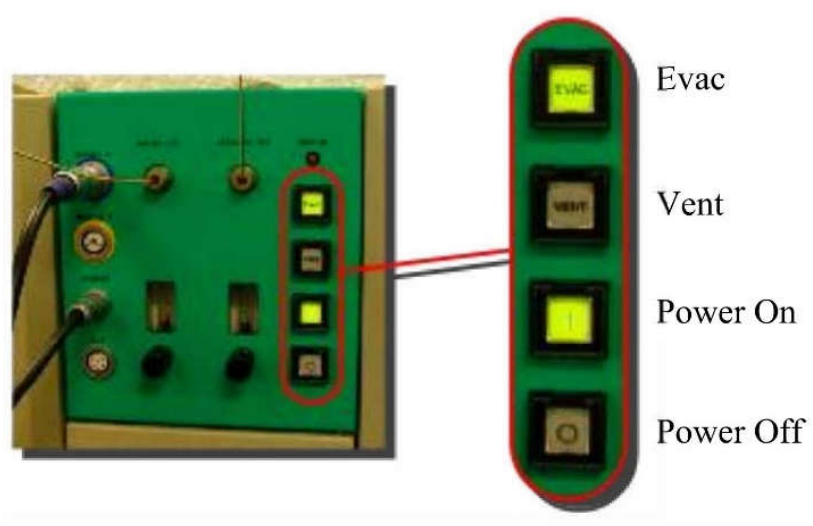

Figure 3. The Front Panel of the AccuTOF ${ }^{\mathrm{TM}}$ 
c. Push the EVAC button to start pumping down the spectrometer. The VENT button will blink until the analyzer reaches a pressure of $\leq 2.0 \times 10-5 \mathrm{~Pa}$. Note: You must wait until the analyzer reaches this pressure before proceeding to the
"Conditioning the MCP" step.

d. Turn on the P.C. and printer.

e. Toggle on the switch on the DART ${ }^{\mathrm{TM}}$ power supply console.

\section{Starting the AccuTOF Instrument Control Software}

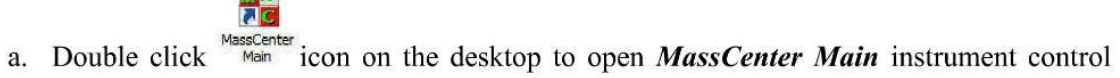
software.

b. Verify that you are in the correct MassCenter project. This information is shown in the top left corner of the MassCenter Main window.

Note: A Project represents the folder where all of your tune methods and data files are stored.

Note: MassCenter automatically loads the most recent project used by the system.

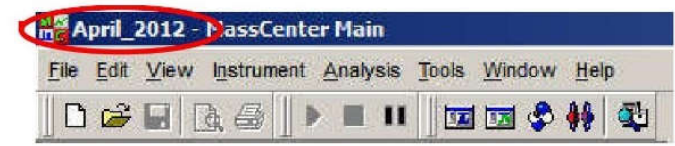

Figure 4. Identifying the Current Project 
c. If you are in the correct Project then skip to the next section Conditioning the MCP.

d. If you are not in the correct project, go to File $\rightarrow$ Open Project and then select the Project that you want to use going forward.

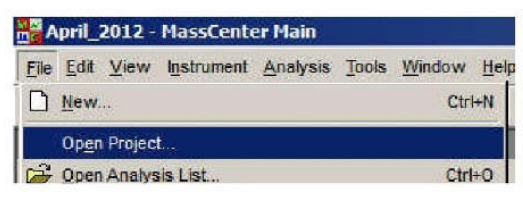

Figure 5. Opening a Different Project

If you need to create a new project please see Appendix D.

\section{Conditioning the MCP}

The Micro Channel Plate (MCP) detector conditioning should always be done after the system has been vented.

a. Open the MS Tune Manager window by clicking Instrument $\rightarrow$ Tune MS .

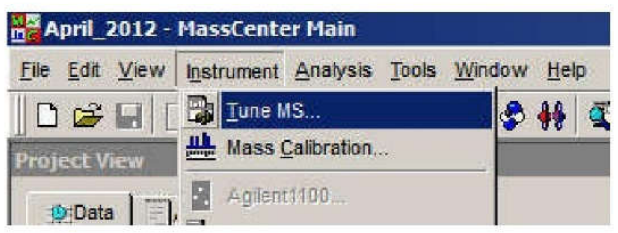

Figure 6. Opening the MS Tune Manager 
b. Click Instrument $\rightarrow$ MCP Conditioning. If this option is grayed out, then the spectrometer has not yet reached an operational vacuum pressure or the MCP conditioning has already been done.

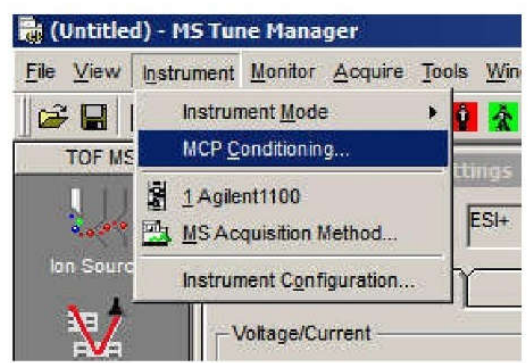

Figure 4. Selecting the MCP Conditioning Option from MS Tune Manager

c. Set the MCP conditioning parameters as shown below or click Use Default and then click Start.

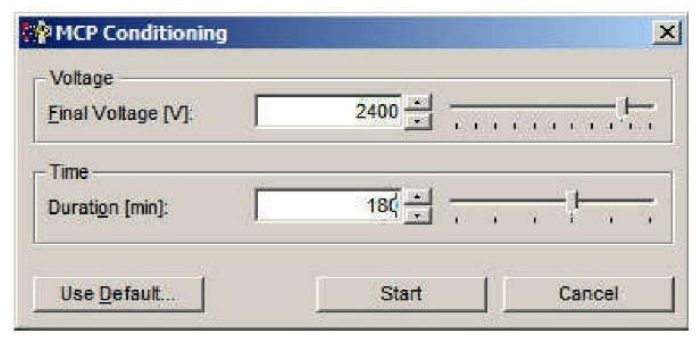

Figure 5. Setting the MCP Conditions

d. When the MCP conditioning is complete, the Close button will be available in the MCP Conditioning progress window. Click Close to exit. 


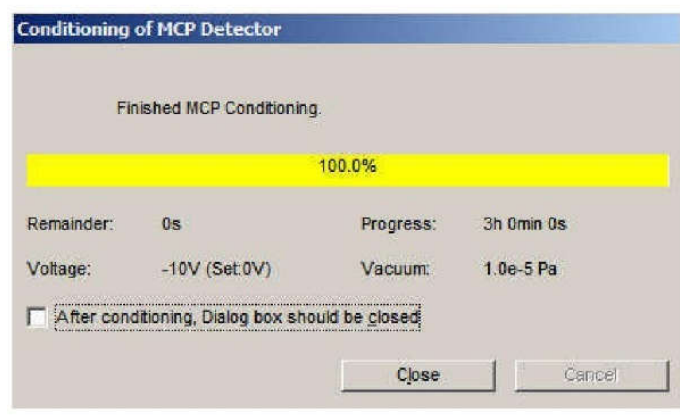

Figure 6. MCP Conditioning Progress Window

\section{Reducing Baseline Noise from FastFlight (Optional)}

This procedure is generally performed by the JEOL Service Engineer during instrument installation and is normally not needed when restarting the system. However, it can be done at any time if there is a question about the magnitude of the baseline noise.

From MS Tune Manager, choose Tools $\rightarrow$ Noise Coefficient of Averager. If this option is grayed out, then you have not yet reached an operational vacuum pressure. Click Start. This process takes 1-2 minutes to complete.

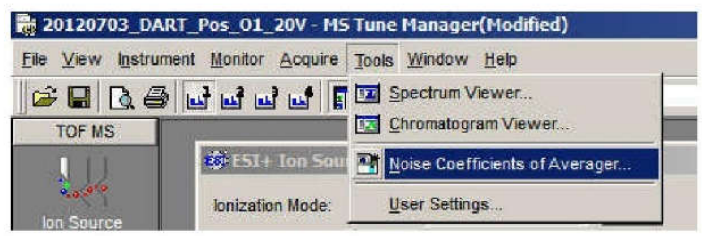

Figure 7. Reducing Baseline Noise from FastFlight 


\section{Turning on the DART ${ }^{\mathrm{TM}}$ Ion Source}

a. Turn on the DART nitrogen and helium gas tanks.

b. Open a web browser and go to 192.168 .10 .111 to access the DART Controller window.

c. In the DART Controller window, left click the Standby button. This will turn on the Nitrogen gas flow.

Note: Nitrogen is the default Standby gas unless otherwise specified.

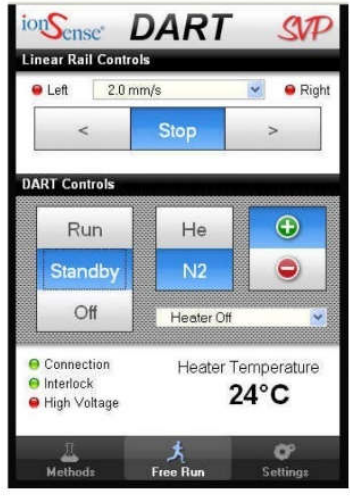

Figure 8. DART Controller Window

d. Select the polarity for the DART analysis by clicking the Plus or Minus buttons. In the case shown above, the Plus button was selected for the DART polarity.

e. Set the DART gas temperature from the drop down menu in the DART Controller window (see below). 


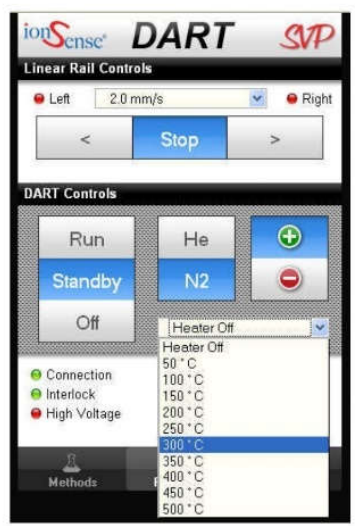

Figure 9. Setting the DART Gas Temperature in Controller Window

f. Once the DART gas reaches the set temperature, click the Run button. This will switch the gas to Helium unless otherwise specified.

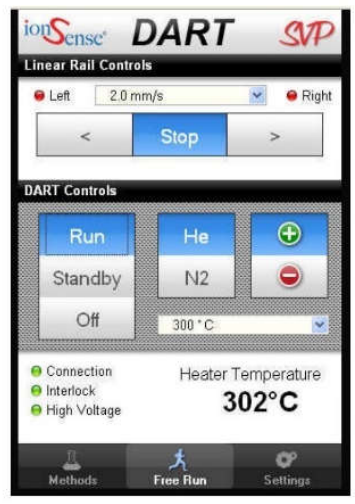

Figure 10. Clicking Run in the DART Controller Window

g. With the exception of setting up the experimental conditions, you are now ready to start a DART $^{\text {TM }}$ analysis. 
JEOL USA, Inc.

\section{Performing a Complete Shutdown}

Use this procedure to completely power down the instrument for long-term periods of time or if you expect a planned power outage.

\section{DART $^{\text {TM }}$ Source Shutdown}

a. In DART Controller window, click the $\boldsymbol{O F F}$ button.

Note: The Standby gas will continue to run until the DART gas temperature cools below $200^{\circ} \mathrm{C}$.

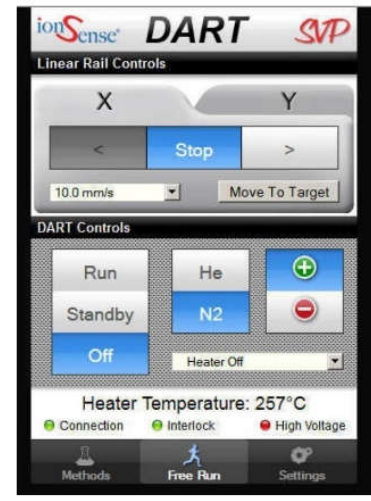

Figure 11. Clicking Off in the DART Controller Window

b. When the Standby gas button is no longer highlighted as active, close the $\boldsymbol{D A R T}$ Controller window. 


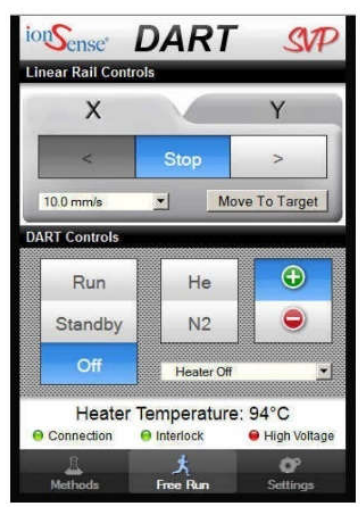

Figure 12. Gas flow has stopped in DART Controller Window

c. Turn off the DART helium and nitrogen gas cylinders.

d. Turn off the switch on the DART power supply console.

\section{AccuTOF $^{\mathrm{TM}}$ Shutdown}

a. In MS Tune Manager, set the instrument mode to Evacuation Ready.

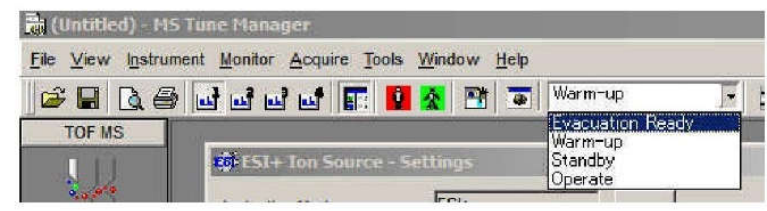

Figure 13. Setting the Instrument to Evacuation Ready 
b. If you were using the ESI source instead of the DART source, then make sure that both the Nebulizing Gas and Desolvating Gas valves are un-checked (turned off) in the ESI+ Ion Source Settings window.

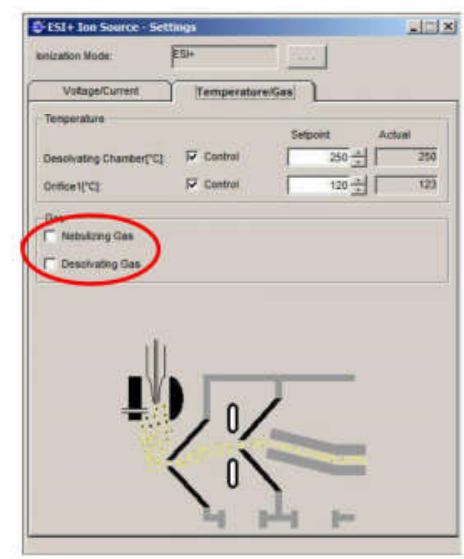

Figure 14. Turning Off ESI Ion Source Gases

c. Close the MS Tune Manager and then exit Mass Center.

d. Make sure that the $\mathrm{N}_{2}$ venting gas pressure is $\geq 600 \mathrm{kPa}$. Look through the grating on the right-hand side of the console to see the red LED gauge. It is not necessary to remove the side panel.

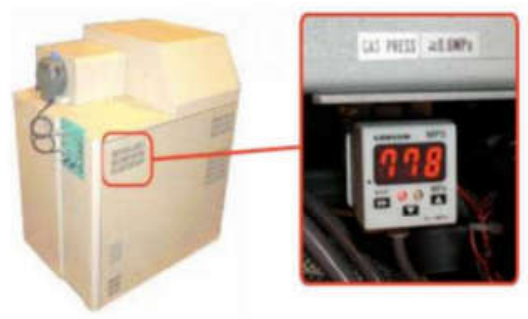

Figure 15. Checking Vent Gas Pressure 
e. Push the Vent switch on the front panel. The Vent light will blink for about 10 minutes indicating that dry nitrogen gas is filling the chamber. Wait until the light stops blinking before proceeding to the next step.

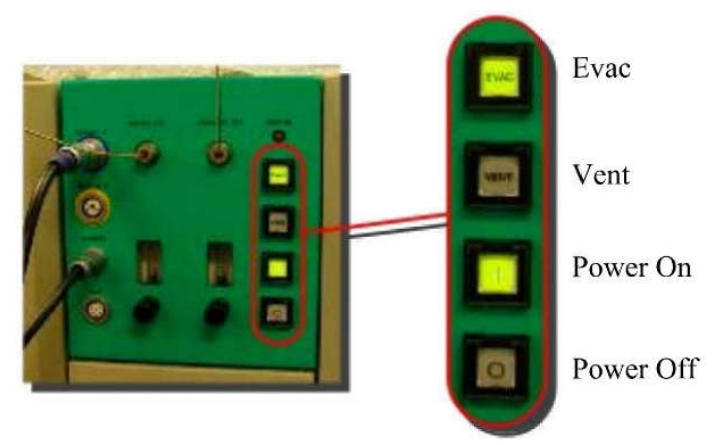

Figure 16. The Front Panel of the AccuTOFтM

f. Turn off the P.C. and printer.

g. Push the Power OFF button on the front panel (see previous figure).

h. Turn off nitrogen gas supply.

\section{Optional}

i. Turn OFF the main breaker switch on the back of the AccuTOFTM mass spectrometer. 
JEOL USA, Inc.

\section{Leaving the Instrument in Idle Mode}

\section{Warm-up Mode}

Use this procedure to leave the instrument in an idle state when you have completed your analyses for the day and will be leaving the AccuTOFTM powered on overnight or over the weekend.

a. In the DART Controller window, click the Off button.

Note: The Standby gas will continue to run until the DART gas temperature cools below $200^{\circ} \mathrm{C}$. This is indicated by the $\mathrm{N} 2$ button remaining blue while the DART temperature cools down.

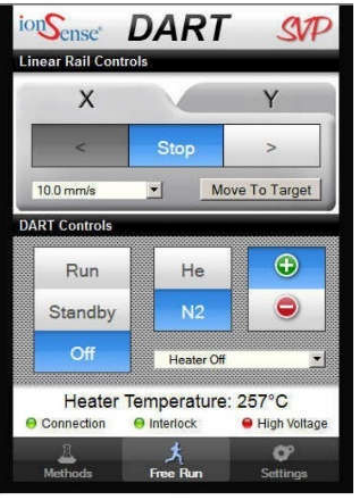

Figure 17. Switching to Off Mode in DART TM Controller Window

b. After the Standby gas button turns gray, close the DART Controller window.

c. Turn off the DART helium and nitrogen gas cylinders.

d. In MS Tune Manager, set the instrument mode to Warm-up. 


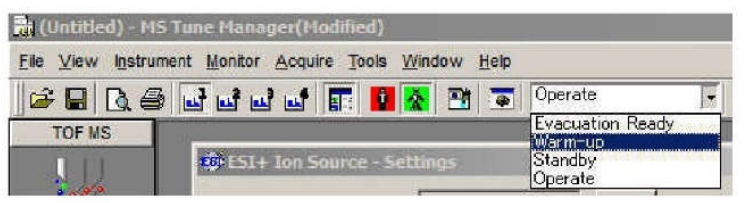

Figure 18. Setting the Instrument to Warm-up Mode

e. In MS Tune Manager, set the detector voltage to $0 \mathrm{~V}$.

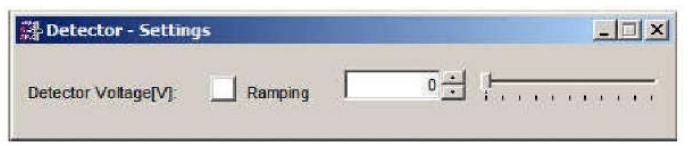

Figure 19. Setting the Detector Voltage to 0

The system is now in an idle state that can be left overnight or over the weekend.

\section{Standby Mode}

Use this procedure when you want to put the instrument in an idle state that is only a few clicks away from being ready for the next sample analysis. Typically, this mode should be reserved for the short time periods between data acquisitions, when you are processing previously collected data or readying new samples for DART analysis.

a. In the DART Controller window, click the Standby button.

Note: This mode switches the DART gas to nitrogen (N2) in order to conserve helium and maintains the set heater temperature. Also, the high voltage for the glow discharge in the DART is turned off. 


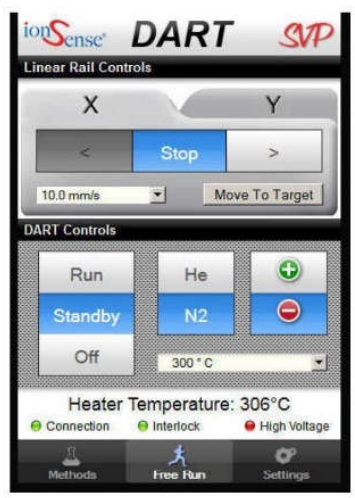

Figure 20. Switching to Off Mode in DART ${ }^{\mathrm{TM}}$ Controller Window

b. In MS Tune Manager, set the instrument mode to Standby.

Note: The Orifice 1 temperature is maintained at the set point in the Ion Source Settings $\rightarrow$ Temperature/Gas tab.

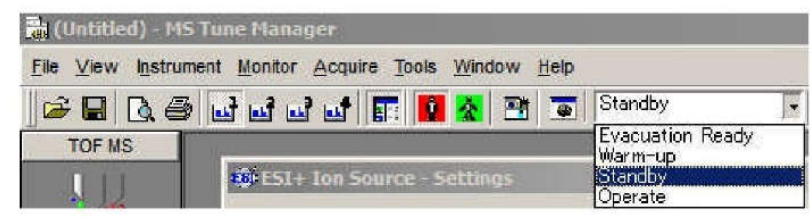

Figure 21. Setting the Instrument to Standby Mode

c. In MS Tune Manager, set the detector voltage to $0 \mathrm{~V}$.

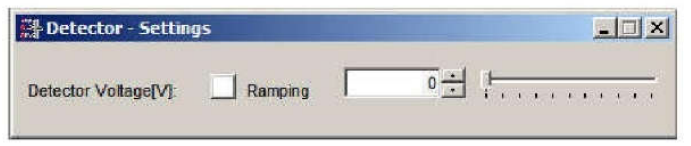

Figure 22. Setting the Detector Voltage to 0

The system is now in an idle state that can be used while you are processing data or readying new samples for DART analysis. 


\section{Collecting a Positive-Ion Mass Spectrum}

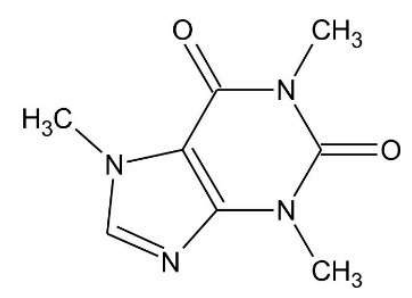

Figure 55. Caffeine FW 194.08

Sample: caffeine $\left(\mathrm{C}_{8} \mathrm{H}_{10} \mathrm{~N}_{4} \mathrm{O}_{2}\right)$ in liquid coffee, $[\mathrm{M}+\mathrm{H}]^{+}$is at $\mathrm{m} / z 195.08820$

External Standard: solution of polyethylene glycol (PEG) 600 in methanol

Sampling Method: glass capillary tube (closed end of a melting point tube)

a. In the MS Tune Manager window select Acquire $\rightarrow$ Start Acquisition to open the Single Acquisition Wizard window.

E0120703_DART_Pos_01_20V - MS Tune Manager
Flie View Instrument Monitor
\begin{tabular}{|c|c|c|}
\hline Acquire Iools Window \\
\hline TOF MS \\
\hline
\end{tabular}

Figure 56. Opening the Single Acquisition Wizard 
b. If you have some special conditions saved, click Load Settings. Otherwise, make sure that this box is unchecked and click Next.

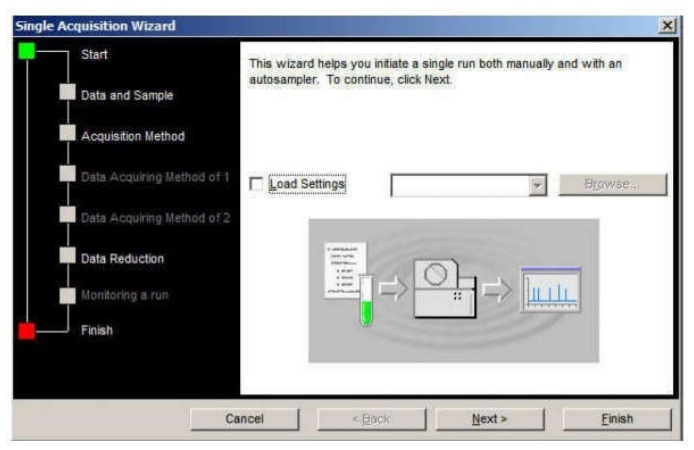

Figure 57. Acquisition Wizard Start Screen

c. Enter a filename in Acquisition Data field and specify a name for the data folder in the Acquisition Data Folder. Typically, the data folder name is a date or perhaps a project code. Optionally, you can also fill in the Sample and Comment fields as well. When you are ready click Next.

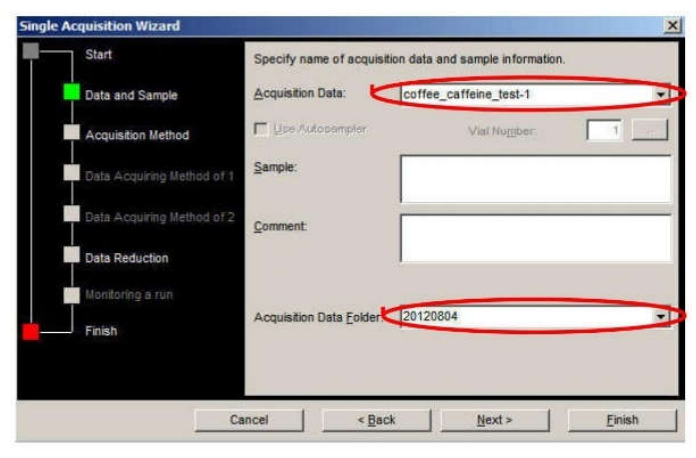

Figure 58. Entering a Filename and Data Folder 
d. In this window you will to decide how the MS Acquisition method will be specified. The most common scenario is to select the Specify new MS acquisition method because it allows you complete control of setting up the data acquisition parameters.

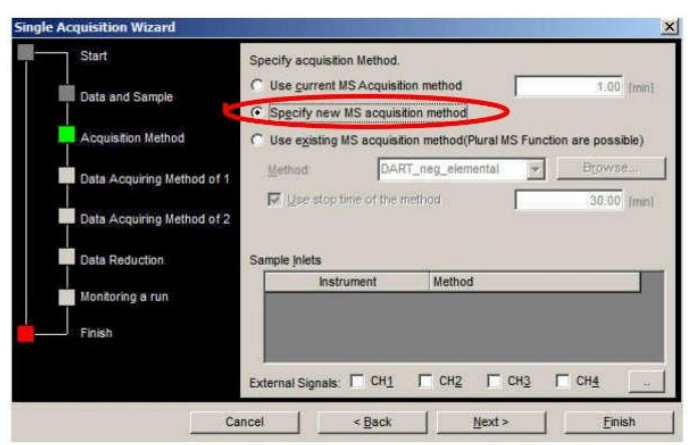

Figure 59. Specifying the MS Acquisition Method

Alternately, you could also select Use existing MS acquisition method to do multifunction switching methods that allow you to do in-source CID at several different cone voltages during data collection. Please see Appendix $\boldsymbol{F}$ for instruction on how to set up a Multi-Function Acquisition Method.

Note: It is generally not recommended that you use the first choice Use current MS acquisition method as you will be unable to customize the data acquisition parameters discussed in Steps e-g.

e. Click Next and then enter an appropriate mass range in the $\boldsymbol{m} / \boldsymbol{z}$ range fields. It is important to remember what your Peaks Voltage was set to in the previous section. For this example, it was set to $600 \mathrm{~V}$ so you will not see many ions below $m / z 60$. 


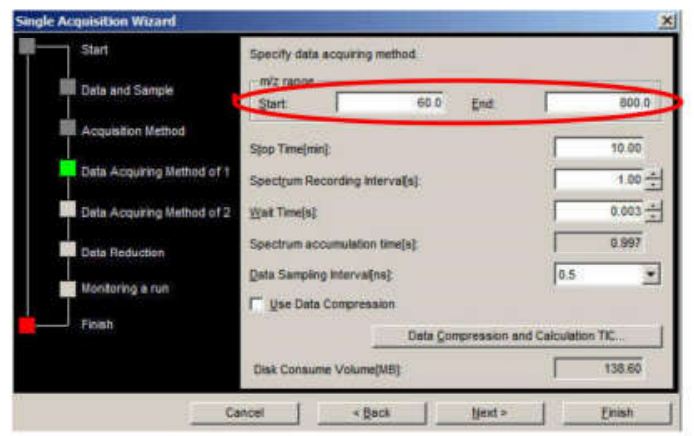

Figure 60. Specifying Acquisition Mass Range

f. Set the Stop Time to a total analysis time value that makes sense for your samples. Generally, a DART analysis for a single sample and the PEG mass calibrant will take less than $5 \mathrm{~min}$. We typically set this time to $10 \mathrm{~min}$ just to be safe.

Note: You will have the opportunity to stop the data acquisition at any time after you have run the samples.

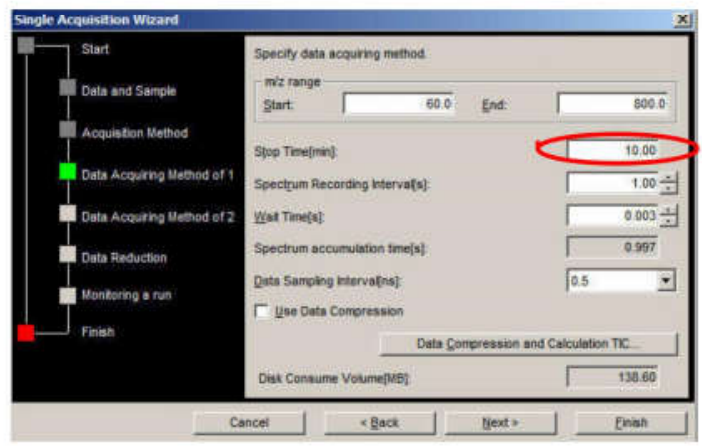

Figure 61. Specifying the Stop Time 
g. You can also set Spectrum Recording Interval in this window. A typical value is 1 or 0.5 $\mathrm{sec} / \mathrm{spectrum}$. The maximum rate is $0.1 \mathrm{sec} / \mathrm{spectrum}$ (or $10 \mathrm{spectra} / \mathrm{sec}$ ).

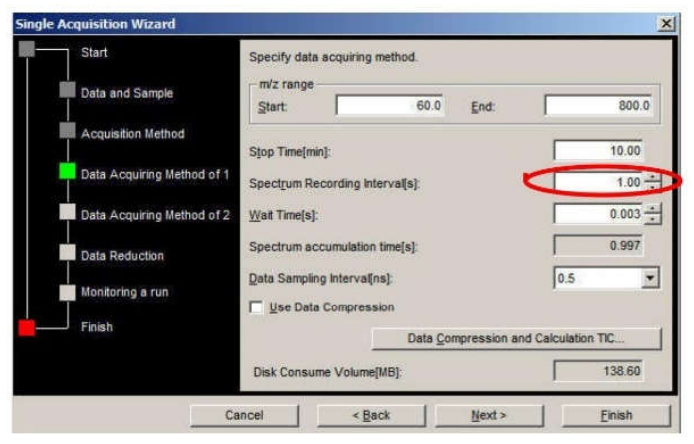

Figure 62. Specifying Spectrum Recording Interval

Note: It is generally not recommended that you change the Wait Time or Data Sampling Interval for normal DART data acquisitions. Additionally, it is not recommended that you use the Data Compression capability.

h. Click Next and then make sure that the Profile radio button is selected.

Note: This choice will allow you to convert back and forth between Profile peaks and Centroided peaks during the data reduction/analysis steps. However, if you select Centroided, then you will NOT be able to look at the Profile peaks. 


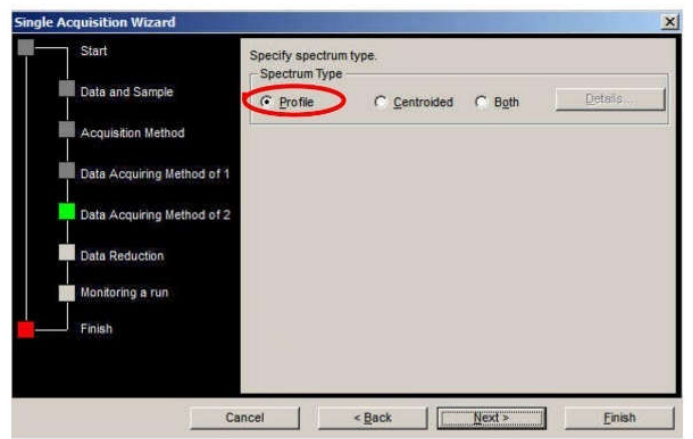

Figure 63. Specifying Profile Data

i. Click Next until you reach the Real-time Options window (i.e. skip over the Data Reduction screen). Make sure that both TIC (total ion chromatogram) and Mass Spectrum are checked. This is important during DART analysis because these selections allow you to see the data in real-time, as it is being acquired.

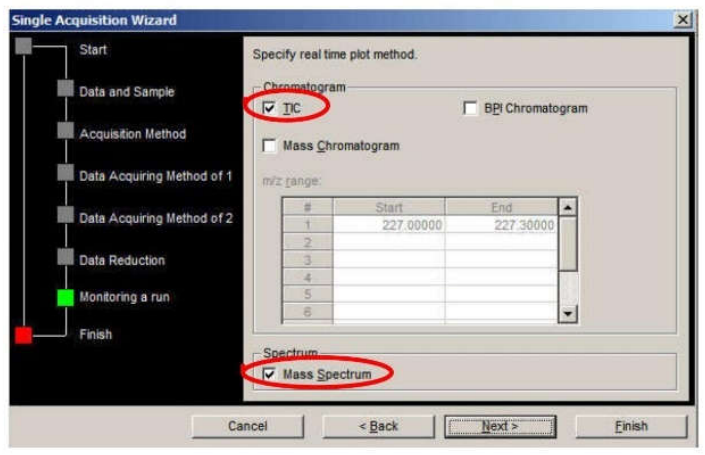

Figure 64. Specifying the Real-Time View Options 


\section{Optional:}

Check the Mass Chromatogram option and enter in a mass range that encompasses a particular mass of interest that you would like to monitor for specifically. In the case shown below, the Mass Chromatogram was set to monitor a small range around $\mathrm{m} / z 195$ so that the response for caffeine $(\mathrm{m} / \mathrm{z} 195.08820)$ could be monitored in real-time.

Note: This choice has absolutely NO effect on the actual recorded data. It is a setting that only affects your ability to see a particular mass chromatogram in real-time, as the data is being collected. You will be able to look at reconstructed ion chromatograms (RIC) for any ion during data reduction/analysis.

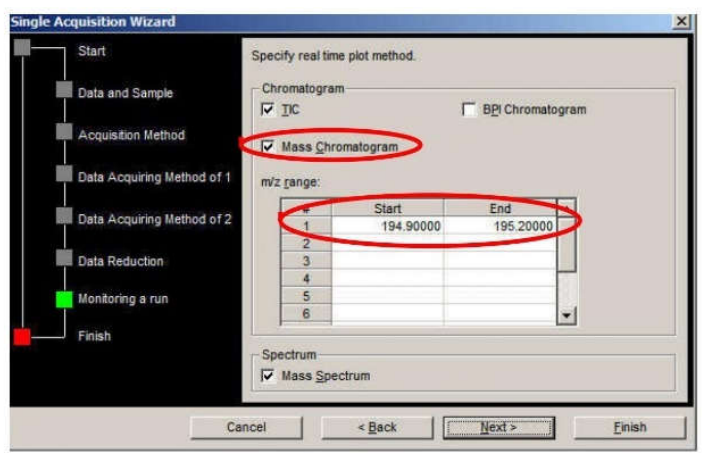

Figure 65. Specifying the Real-Time Mass Chromatogram

j. Click Next and then click Finish to close the wizard.

k. If you specified a new data folder in Step c, then a dialog box will appear asking if you want to create a new folder. Click Yes. You should now see the Spectrum Viewer and Chromatogram Viewer windows appear on the screen along with a Start Run dialog box. Generally, both viewer windows should be arranged so that you can see them both on the computer screen at the same time (see the example below). 


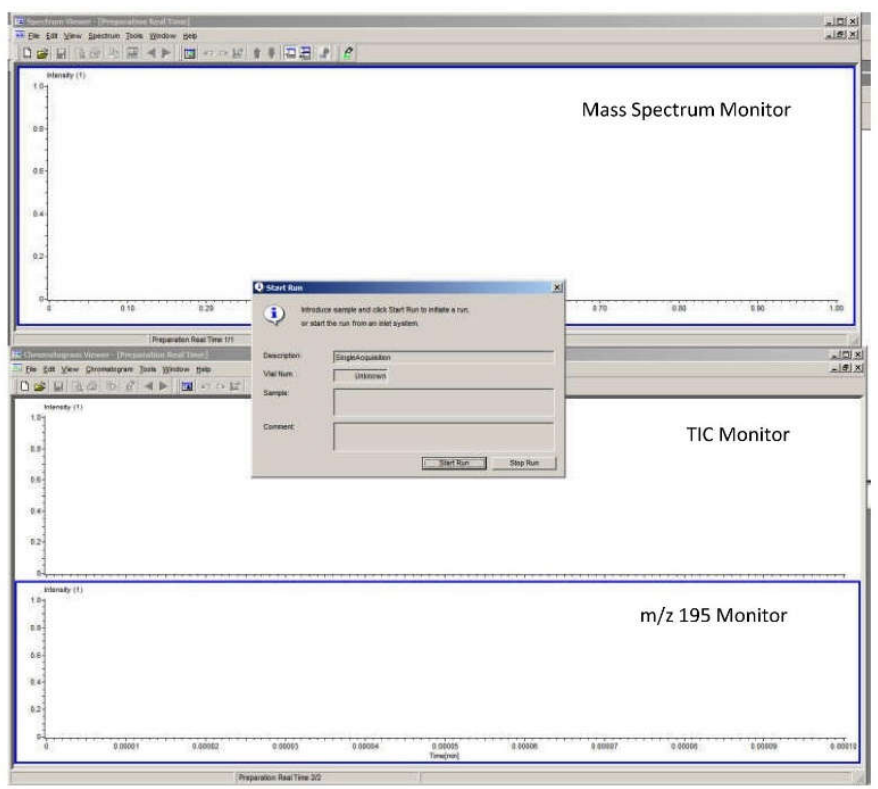

Figure 66. Arrangement of Viewer Windows during Data Acquisition

Note: In this case, there are 2 chromatograms that appear in the Chromatogram Viewer window, one for the TIC and one for $\mathrm{m} / \mathrm{z}$ 195. If you did not choose to monitor for a particular mass, then only the TIC would be present in this window.

1. At this point, you should verify in the DART Controller window that the Run button is highlighted blue and that the temperature is at the set point $\left(300^{\circ} \mathrm{C}\right.$ in this case). 


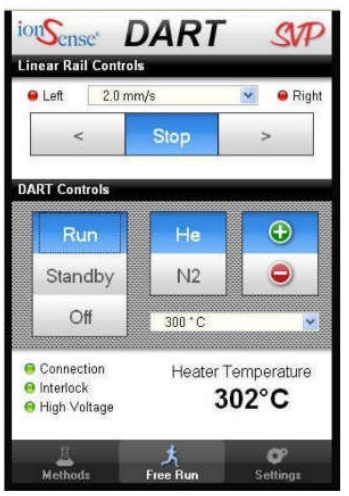

Figure 66. Turning on the DART for Analysis

m. When you are ready to start the measurement, click the Start Run button in the Start Run dialog window.

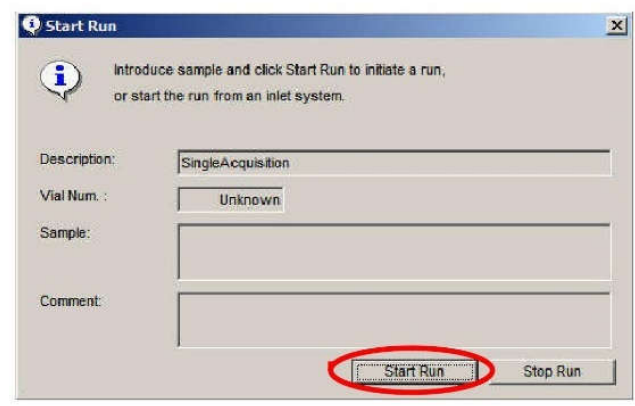

Figure 67. Click Start Run to Begin the Analysis

n. Dip the closed end of a melting point tube in the PEG solution and then dangle it in the gap between the DART ion source and the AccuTOF atmospheric pressure inlet (API), taking care not to completely block the He gas flow (as shown below). 


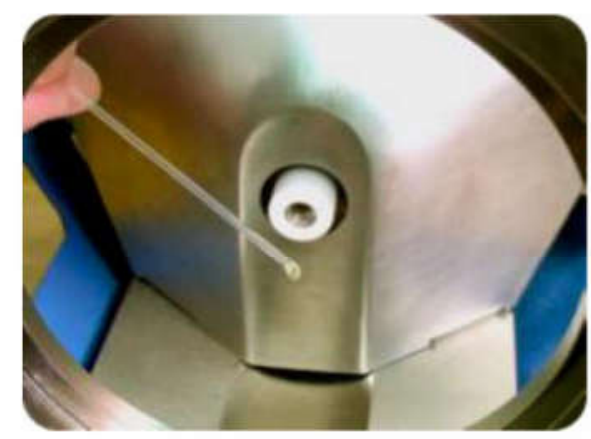

Figure 66. Dangling a Capillary Tube in the Sample Gap

You should see a series of PEG peaks appear in the Spectrum Viewer and a change in the intensity of the TIC in Chromatogram Viewer. It is useful to note at roughly what time the peak intensity changes in Chromatogram Viewer. This will make it easier to find the PEG peak when processing. The figure below shows a typical PEG spectrum. You should repeat the sampling process a few times to ensure that you have a strong PEG signal recorded in the data file.

Note: It is important that the PEG peaks extend out over the mass range of interest.

Therefore, the sample should be held in the DART gas stream long enough to heat the sample so that the PEG masses extend across most of the recorded mass range.

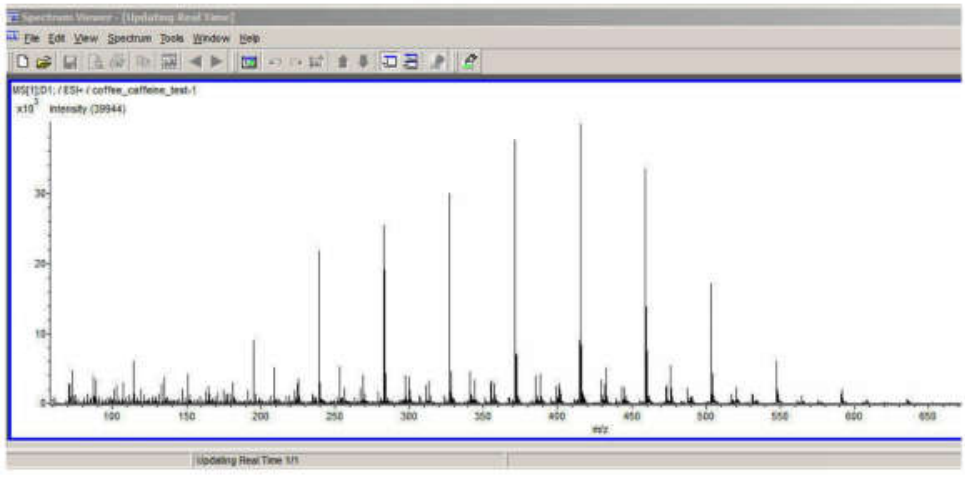

Figure 67. Real-time Spectrum Monitor of PEG 600 Standard 
o. Once you are done sampling the PEG, dip the closed end of a fresh melting point tube into the brewed coffee so that there is a coating of liquid on the tip. Again dangle the melting point tube into the gap. You should see a caffeine peak at $\mathrm{m} / z 195$, along with a number of other masses from the coffee matrix, appear in the Spectrum Viewer. Additionally, you should see a change in the intensity of the TIC and $\mathrm{m} / \mathrm{z} 195$ mass chromatogram in the Chromatogram Viewer. It is recommended that you visually note at roughly what time the peak intensity changes in the Chromatogram Viewer.

Note: You should repeat the sampling process a few times to ensure that you have a strong caffeine/coffee signal recorded in the data file.

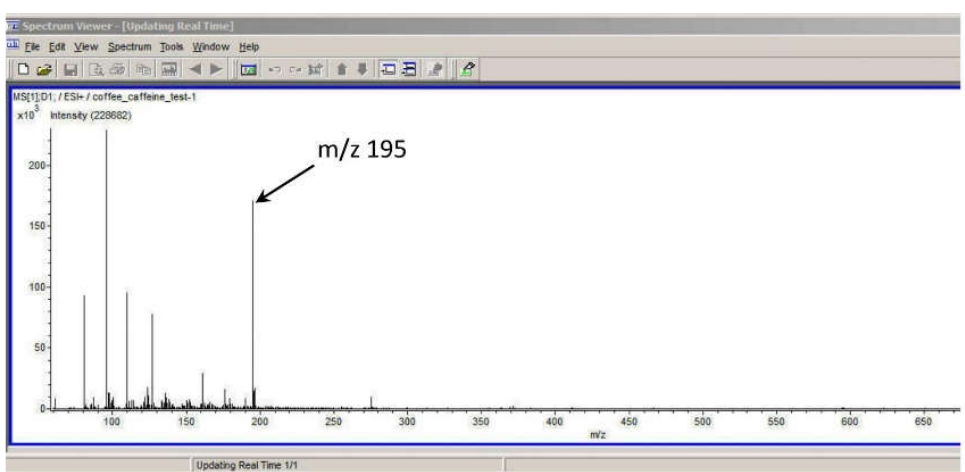

Figure 67. Real-time Spectrum Monitor of Caffeine in Coffee

p. Once you are done analyzing the sample, click the Stop button in MassCenter Main.

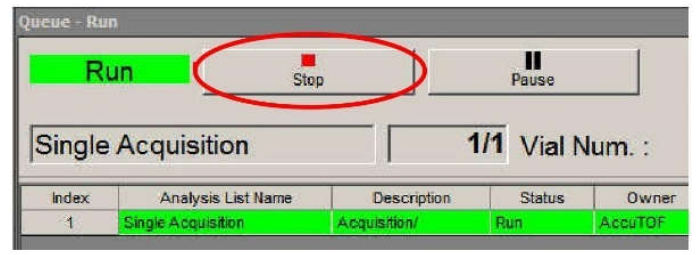

Figure 68. Stopping the Acquisition in MassCenter Main 
q. Set the DART Controller to Standby mode to conserve helium.

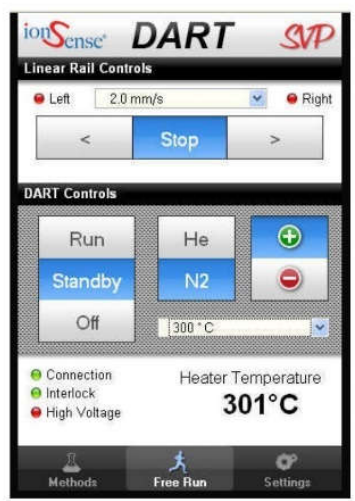

Figure 69. Putting DART in Standby after Data Acquisition

r. Change the instrument mode to Standby.

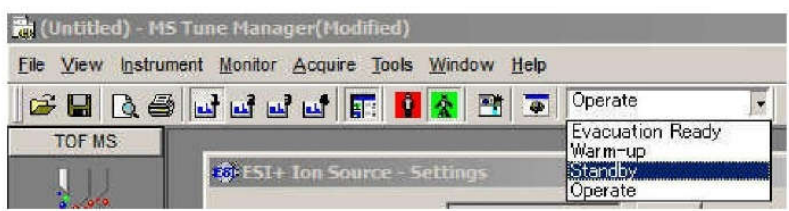

Figure 70. Changing the Instrument Mode to Standby

s. In the Detector-Settings window set the voltage to $0 \mathrm{~V}$.

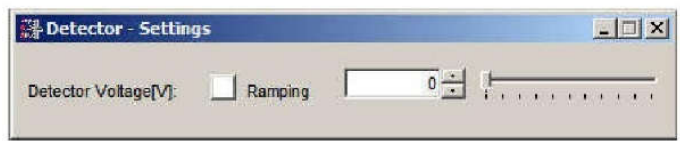

Figure 71. Setting the Detector Voltage

The data is now ready to be processed using data reduction/analysis software. 
VITA

TORKI A. ZUGHAIBI

1987

Born, Austin Texas

2005-2009

B.S., Forensic Science

Track, Forensic Chemistry

Minor, Chemistry

Virginia Commonwealth University

Richmond, Virginia

(Cum Laude)

2006

Undergraduate Teaching Assistant

Virginia Commonwealth University

Richmond, Virginia

2009-2011

M.S., Forensic Science

Track, Trace

Virginia Commonwealth University

Richmond, Virginia

2011

Research Internship

Virginia Department of Forensic Sciences

Controlled Substances Section

Richmond, Virginia

2012-2017

Doctoral Candidate

Florida International University

Miami, Florida

2014-2017

Lecturer

King Abdul-Aziz University

Rabigh, Kingdom of Saudi Arabia 


\section{PUBLICATIONS AND PRESENTATIONS}

T.A. Zughaibi, R. Steiner, M. Miller. Forensic Analysis of Carpet Fiber Samples Using DART-AccuTOF. In Preparation.

T.A Zughaibi, K.G Furton, H.K Holness. Study of the Transport of Narcotic Odorants using DART-AccuTOF. In Preparation.

T.A Zughaibi, K.G Furton, H.K Holness. Analyzing Controlled Odor Mimic Permeation Systems for Possibility of Cross Contamination using Direct Analysis in Real Time Mass Spectrometry. In Preparation.

T.A Zughaibi, K.G Furton, H.K Holness. Absorption of Odorants onto Common Materials using Direct Analysis in Real Time Mass Spectrometry. In preparation.

T. Zughaibi, R. Steiner, M. Miller. Forensic Analysis of Carpet Fiber Samples Using DART-AccuTOF. Poster session presented at the annual meeting of the American Academy of Forensic Sciences, Washington D.C. February 2013 